\title{
Magnetotellurics and Geomagnetic Depth Sounding in Queensland, South Eastern Australia -Evidence for the Tasman Line?
}

vorgelegt von

Fon Lawrence Teku

aus Bessi-Batibo/Cameroon 
D7

Referent:

Prof. Dr. Andreas Hoerdt

Korreferent:

Prof. Dr. Cynthia Volkert

Tag der mündlichen Prüfung: 


\section{Contents}

1 Introduction 1

2 Theory of electromagnetic methods $\quad 9$

2.1 EM signal source . . . . . . . . . . . . . . . . . . . . . . . . . 9

2.2 Maxwell's equations . . . . . . . . . . . . . . . . . . . . . . . 14

2.3 Magnetotellurics . . . . . . . . . . . . . . . . . . . . . . 16

2.3 .1 Two dimensional problem . . . . . . . . . . . . . . . . . 18

2.3 .2 Induction arrows . . . . . . . . . . . . . . 20

2.3.3 Distortion and dimensionality analysis of MT transfer functions . . . 22

2.3 .4 Magnetic distortion . . . . . . . . . . . . . . 28

2.3 .5 Dimensionality analysis . . . . . . . . . . . . . . . . 30

2.4 Geomagnetic depth sounding . . . . . . . . . . . . . . . . . . 31

2.5 Current channelling distortion analysis . . . . . . . . . . . . . . 41

3 Previous work and description of study area $\quad 45$

3.1 Seismic tomography results . . . . . . . . . . . . . . . . . 53

3.2 Gravity surveys . . . . . . . . . . . . . . . . . . . . . . . . . . . 54

3.3 Magnetic anomaly data ... . . . . . . . . . . . . . 56

3.4 Geomagnetic field Fluctuations over Australia and Coast effects . . . . . . . 58

3.5 Previous electromagnetic array studies . . . . . . . . . . . . . . 60

3.6 Tasman line and recent interpretation . . . . . . . . . . . . . . 62

4 Field measurements in Central and East Australia $\quad 67$

4.1 Data processing . . . . . . . . . . . . . . . . . . 6 68

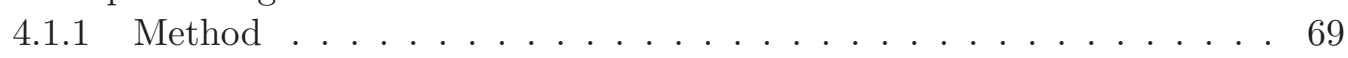

4.2 Error estimations . . . . . . . . . . . . . . . . . . . . . . 69

4.2 .1 Results $\ldots \ldots \ldots \ldots \ldots$

4.2 .2 Conclusion . . . . . . . . . . . . . . . . . . . 74

4.3 MT results . . . . . . . . . . . . . . . . . . . . . . . . 74

4.3 .1 Induction vectors . . . . . . . . . . . . . . . . . . 83

4.3.2 Strike direction and phase sensitive skews . . . . . . . . . . 88

4.4 GDS results . . . . . . . . . . . . . . . . . . . . . . . . . 92

4.4 .1 GDS strike directions $\ldots \ldots \ldots$. . . . . . . . . . . . . 96

$\begin{array}{lll}5 & \text { 3D modelling of the data } & 101\end{array}$

5.1 3D modelling of the Australian data . . . . . . . . . . . . . . . . 102

5.2 Results from the model compared with measured data . . . . . . . . . . . 107

5.2 .1 GDS results . . . . . . . . . . . . . . . . . . . 107

$5.2 .2 \quad$ MT results . . . . . . . . . . . . . . . . . . . . 117

5.3 Structural \& geological implications of the model . . . . . . . . . . . . . . 122 
5.3 .1 Sediments of the Eromanga Basin . . . . . . . . . . . . . . 123

5.3 .2 Resistive structures in and around the Mt. Isa block . . . . . . . 126

5.3 .3 Carpentaria conductivity anomaly . . . . . . . . . . . . 127

5.3 .4 Tasman Line . . . . . . . . . . . . . . . . . . . . . . . . . . 129

$5.3 .5 \quad$ Eastern Australia . . . . . . . . . . . . . . . . . . . . . . 133

$\begin{array}{lll}6 & \text { Conclusions } & 135\end{array}$

\begin{tabular}{ll}
\hline A Telluric and magnetic tensor decomposition & 139
\end{tabular}

\begin{tabular}{lr}
\hline B Field sites and data & 141
\end{tabular}

B.1 Names and locations of MT sites . . . . . . . . . . . . . . . . . . 141

B.2 Processed MT data . . . . . . . . . . . . . . . . . . . . . . . 142

$\begin{array}{lr}\text { C Explicit expressions for transfer functions } & 151\end{array}$

C.1 Magnetotellurics . . . . . . . . . . . . . . . . . . . . . . . . . . 151

C.2 Geomagnetic Depth Sounding-GDS . . . . . . . . . . . . . . . 152

C.3 Göttinger Data Format . . . . . . . . . . . . . . . . . . . 153

C.3.1 *.mtt-Format . . . . . . . . . . . . . . . . . . . . 153

C.3.2*.gdt-Format . . . . . . . . . . . . . . . . . . 153

$\begin{array}{ll}\text { D Programs used } & \mathbf{1 5 5}\end{array}$

D.1 Programs to analyse and display data . . . . . . . . . . . . 155

D.1.1 emdisp . . . . . . . . . . . . . . . . . . . . 155

D.2 3D modelling program $\ldots \ldots \ldots \ldots \ldots \ldots$

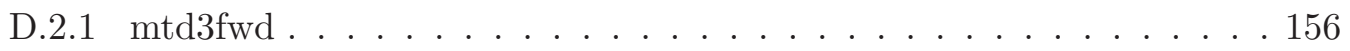

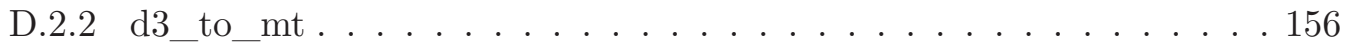

D.2.3 d dtogmt . . . . . . . . . . . . . . . . . 156

D.3 Data manipulating programs . . . . . . . . . . . . . . . 156

D.3.1 Convert . . . . . . . . . . . . . . . . . . 156

D.3.2 Calc . . . . . . . . . . . . . . . . . . . . . . . . 157

D.3.3 Gdsswap . . . . . . . . . . . . . . . . . . 157

$\begin{array}{lr}\text { E Phase tensor } & 159\end{array}$

$\begin{array}{ll}\text { References } & 161\end{array}$

\begin{tabular}{ll}
\hline Acknowledgements & 177
\end{tabular}

\begin{tabular}{ll}
\hline Curriculum Vitae & 179
\end{tabular} 


\section{Introduction}

Electrical conductivity is an important physical property of rocks. It varies both laterally and vertically in our conducting Earth (Haak and Hutton, 1986). It can be thoroughly examined by observing and so exploiting the processes of natural electromagnetic induction which occur in both the Earth and oceans. Electromagnetic methods (electromagnetic depth soundings) consequently use natural fluctuating electric and magnetic fields which induce currents in the Earth and its oceans to resolve conductivity structures within the subsurface (Earth's interior). This is possible because the magnitude, direction, and depth of penetration of the induced currents are determined essentially by the distribution of electrically conducting materials in the Earth (Campbell et al., 1997).

There are two powerful methods of electromagnetic depth soundings that can be used to probe the subsurface conductivities, due to the fact that inductive coupling of fields contain information about the electrical conductivity distribution at crustal and upper mantle depths or even beyond. (i) the geomagnetic depth sounding (GDS) and (ii) the magnetotelluric (MT) method. These two methods are powerful complementary techniques for investigating the electrical conductivity structure of the Earth.

In the geomagnetic depth sounding method, the separation of the time-varying magnetic fields of external origin, from those associated with induced currents flowing within the conducting Earth is exploited. In this case, the horizontal and vertical components of the magnetic field is ultilised to obtain a perturbation tensor, $\underline{\underline{W}}$ (Schmucker, 1970) that relates the magnetic field components at a measurement site ${ }^{1}$ to those at a reference or normal site ${ }^{2}$. Information about the conductivity structure is then obtained from the perturbation tensor.

Previous GDS analysis concerntrated in studying anomalies in the vertical component of the magnetic field by analysing induction vectors only, however, nowadays there is an obvious substantial advantage in studying anomalies in the horizontal magnetic components in combination with anomalies in the vertical component to locate conductivity contrasts. Over the centre of a conductor, anomalies in the horizontal component have a maximum and fall rapidly to zero as one moves away from the conductor. In contrast, anomalies in the vertical component are zero immediately above a conductor and increases to a maximum at the edges of the conductor. This concept will be elaborated in section 2.4. On the other hand, the main focus of MT is the measurement of the time variations of horizontal orthogonal components of natural electric and magnetic fields, since conductivity changes within the Earth alter the amplitude and phase relationships between the electric and magnetic fields that a magnetometer registers at the surface compared with the relationship expected on the surface of a homogeneous conductivity half-space. Here, components of the induced electromagnetic field measured at a single site are used to

\footnotetext{
${ }^{1}$ would be denoted in this thesis by subscript a

${ }^{2}$ would be denoted in this thesis by subscript $n$
} 
compute a period-dependent impedance tensor $\underline{\underline{Z}}$ from which apparent resistivities and phases can be obtained (more in sections $2.2 \& 2.3$ ).

These methods can be used together with other geophysical methods to better constrain the nature of the crust and mantle beneath the Australian plate that is made of different tectonic blocks and boundaries and whose evolution is not yet well understood. Many studies have shown that older rocks are less conductive than younger ones; conductivity imaging using long period MT and GDS soundings can therefore be used to separate the less conductive Precambrian /Proterozoic zones from the more conductive Phanerozoic zones. If this separation is possible, then it is of course also possible to use these methods to delineate a boundary between Precambrian/Proterozoic and Phanerozoic zones. This is the case because MT and GDS distinguishing features of the deep crust are often continuous or change slowly across a single terrane. However, MT and GDS characteristics will show sudden contrasts across terranes with different features and other major tectonic boundaries. Features such as conductivity contrast across tectonic boundaries, the depth of the Moho (boundary between the crust and mantle), the sudden change in conductivities across the Moho, the resistivity profile across the crust and the resistivity of the upper mantle may be delineated out using electromagnetic sounding methods. The imaging (mapping) of deep rooted structural distinguishing features using electromagnetic induction methods allows the lateral extent of a given terrane and location of terrane boundaries to be determined (Brown \& Whelen, 1995; Korja, 1997; Jording et al., 2000; Selway, Heinson \& Hand, 2006). In general, the consistency of deep structures within a single terrane and the contrast of deep structures across terrane boundaries can be illustrated using electromagnetic induction methods. Understanding the composition of the lower crust and mantle of continental regions is crucial in understanding the large scale processes leading to the development of our continents (O'Reilly et al., 2001). The development and evolution of the Australian continent is still not well understood especially in relation to previous supercontinents.

In order to unravel the uncertainty surrounding its evolution and geology, recent research and debate have focused on the position and the timing of the Rodinia break-up. Wingate et al. (1998) as well as Wingate and Giddings (2000), pointed out the break-up time to be around 780 Ma. Despite recent evidence regarding the rifting of the supercontinent Rodinia, the position and nature of the estimated $780 \mathrm{Ma}$ continental margin of the Gondwanan fragment in eastern Australia remains controversial. This led to the idea of a Tasman Line first proposed by Hill (1951); more rigorous discussion on this issue will be presented later.

What is generally accepted about the continent today is the existence of the supercontinent Rodinia (the late Mesoproterozoic to late Neoproterozoic supercontinent formed by the assembly of Precambrian terrains of Australia, North America-Canada, India, Madagascar, Sri Lanka, and East Antarctica (Cengage, 2003, 2006)). In addition, there is no dispute about the rifting of Rodinia-Gondwana as well as the fact that the unconcealed geology of the Australian continent is composed of an assemblage of crustal blocks that can be broadly grouped into the Precambrian western and central cratons(long-lived stable parts of continents) and the Phanerozoic eastern Australia (Direen \& Crawford, 2003).

The evolution of the Phanerozoic east Australia remains highly sepeculative. Rocks of the Orogens constituting Phanerozoic east Australia has deformed and metamorphosed. 
Orogenic processes alter pre-existing crust i.e. orogens destroy prior geologic information via metamorphism, erosion and subduction (Evans, 2009). Metamorphism modifies the character of pre-existing rocks beyond recognition through remobilization and resets radiometric clocks (history.com). Structural differences between the Precambrian shield and Phanerozoic eastern Australia are deduced from surface wave dispersion (Muirhead and Drummond, 1991; Denham, 1991) and teleseismic travel-time residuals (Drummond et al., 1991) whose origin is due to structures that certainly extend below $100 \mathrm{~km}$ depth.

The boundary between the Proterozoic western and central Australia on one hand and the Phanerozoic east resulting from the Rodinia breakup on the other is termed the Tasman Line (Scheibner and Veever 2000; Gunn et al. 1997; Scheibner 1998, 1996; Cengage, 2003, 2006; Cayley et al., 2011). The idea of the Tasman line remains unresolved by both geologists and geophysicists. Until now, it can be regarded as a conceptual boundary between the older, Proterozoic and Archaean region in west and central Australia on one side and the younger (Phanerozoic) rocks of east Australia on the other side.

As mentioned earlier, the term Tasman Line was first coined by Hill (1951) when he defined the position of the boundary between Precambrian west and centre and Phanerozoic east from geological investigations. His map of the Tasman line delineated it running east of the Great Artesian Basin and into New South Wales at longitude $144.5^{\circ} \mathrm{E}$ (Direen and Crawford, 2003). Until now, nothing concrete has been established about the Line due to its obscurity under later sedimentary sequences which act as an electrical screening effect along much of its length and thus is still a topic of further research.

Hill's definition of the Tasman Line was accepted by Brown et al. (1968), however, they delineated two alternative western boundaries for the southern 'Tasman orthogeosyncline', to be either through western Victoria, or through the Fleurieu Peninsula in southern Australia (Direen and Crawford, 2003). Later on, other plate tectonic researchers concurred with the concept of the Tasman Line (Harrington 1974; Scheibner 1974). However, the Tasman Line was redefined to symbolise the boundary between the not too severe deformed rocks of the 'Tasman Fold Belt System' (Scheibner 1974, Direen and Crawford, 2003) and older established cratonic blocks to the West. Drummond et al. (1998) suggested that the Tasman Line was a broad zone of deformation. Milligan, Petkovic \& Drummond. (2003) analysed magnetic and gravity data and obtained two different positions for the Tasman Line. Beause each dataset gave a different position for the line, they pointed out that it is difficult to resolve structural information interpreted from different data types.

To make the concept of the Tasman Line more ambiguous, Direen et al. (2003) after a detailed review of geological and geophysical potential field evidence suggesting that the lineaments associated with the Tasman Line resulted from a number of events of different ages, proposed that the notion of the Tasman Line in south-eastern Australia should be simply put aside as misleading and unhelpful for the important debate on the whereabouts of the Rodinia break-up margin. They argued that a new definition of the Tasman Line crops up when a new idea emerges. Making reference to Direen (1998) and Buckley (2001), they attributed the source of magnetic anomalies around the Tasman Line to beds of magnetic ironstone, phyllite and metabasite within the deformed Ponto Group, a series of multiply deformed, steeply east-dipping thrust slices of psammites, phyllites and deformed tholeiiitic volcanics (Direen and Crawford., 2003). To further undermine the issue, they suggested that the main locus of Rodinia break-up boundary may have 
occurred elsewhere-probably well to the east or that the estimated 780 Ma Rodinia breakup may not have occurred at all.

Although Direen and colleague dismissed the concept of the Tasman Line, their paper (Direen and Crawford., 2003) added a wealth of new knowledge on how the concept can be better approached. Nonetheless, their insightful and vigorous arguments almost dampen the spirit of those interested in the concept of the Tasman Line until Lilley et al. (2003) used electromagnetic depth sounding data and provided evidence for a contrast in the deep crust between older Proterozoic rocks to the west and younger Phanerozoic terrane to the East. This contrast between the two terranes became very evident again right down into the deeper mantle as was clearly shown in seismic data (Kennett et al., 2004). All these rekindled the concept of the Tasman Line again. In a most recent paper by Cayley et al. (2011), they referred to the Tasman Line as a passive margin setting along the eastern margin of the Precambrian Gondwanaland craton that developed during Proterozoic Rodinia break up.

Moreover, the concept has remained ambiguous and inconclusive because magnetic and gravity anomalies used in the definition of the various Tasman Lines as outlined above lie in the upper part of the crust. But geophysical evidence for a contrast stemming from the edge of the Australian Shield cannot limited to the near-surface. In addition, if the Tasman Line is obscured under later sedimentary cover along much of its length, any probes to unearth it must extend to greater depths. The concept of the Tasman Line will be elaborated and broadened in section 3.6.

As already stated above, electromagnetic methods offer a better and quite elegant opportunity to study the subsurface at greater depths since lithospherical compositions and ages are often associated with distinct conductivities (Boerner et al., 1999). Therefore, electromagnetic induction data have the ability to delineate large scale structural features of the continent and can be used to determine the controversial Tasman Line (boundary between Precambrian west and Phanerozoic Eastern Australia). Furthermore, it is now well known that terrane boundaries are associated with conductivity anomalies (Jording et al., 2000; Brown \& Whelan, 1995 ); major conductivity anomalies marked old plate margins(Gough, 1983) which can be located and imaged by long period MT or GDS soundings; some anomalies are located at plate boundaries where tectonic activity is very high(Honkura, 1978). Brown \& Whelan (1995), Korja (1997), Boerner et al. (1999); Jording et al. (2000) \& most recently Miensopust et al. (2011) have used EM sounding to delineate terrane boundaries across their respective regions of study. Jording et al. (2000), in order to emphasize how efficient it is to apply EM sounding methods to map terrane boundaries had this to say "We conclude that basic MT data without any elaborate model calculation may be used to map different crustal units and may help to define terranes and their boundaries in conjunction with geological observations". Therefore motivated by the work of these persons, this thesis seeks to exploit the process of electromagnetic induction methods and increase our understanding of the electrical conductivity structure of Queensland's subsurface via its inductive response to external field variations with a focus on delineating the controversial Tasman Line in eastern Australia and also mapping out details of the Carpentaria and Southwest Queensland anomalies.

After Parkinson's pioneer work on electromagnetic induction (Parkinson, 1959), the electromagnetic depth sounding method continues to be a versatile tool to study the elec- 
trical conductivity structure of the Earth. The method has been used to investigate the substratum's electrical conductivity of Australia and other continents. The first major magnetometer array study in Australia was by Gough et al.(1972, 1974). They unearthed the Flinders Anomaly found in the Adelaide Geosyncline of South Australia (figure 1.1). Most campaigns that followed were sparsely scattered magnetometer array studies across the continent to investigate induction arrows, transfer functions between local horizontal and vertical magnetic fields (Woods and Lilley, 1979, 1980; Chamalaun and Barton, 1993; Chamalaun et al., 1999). Chamalaun and Barton (1993), interpreted mainly induction arrows and concluded that the broad conductivity structure of Australia appears to be dominated by the conducting sediments of the major sedimentary basins ${ }^{3}$. They suggested a narrow band of possibly interconnected conductors, known as the Intracratonic Conductive Zone (figure 1.1). Three separate conductivity anomalies define what Chamalaun and Barton, (1993) referred to as the Australian intracratonic zone. The first one is the Southwest Queensland Anomaly (Woods and Lilley 1979, 1980) that is probably connected to the Arckaringa Anomaly further south. The second conductivity Anomaly is the Canning Basin Anomaly in the west, which was mapped by the 1985 magnetometer array of Chamalaun and Cuneen (1990) and the third is what is now commonly known as the Carpentaria Anomaly in North Queensland. Figure 1.1 shows the intracratonic zone as mapped out by Chamalaun and Barton (1993).

\footnotetext{
${ }^{3}$ Eromanga, Carpentaria and Surat basins that make up the Great Artesian Basin
} 

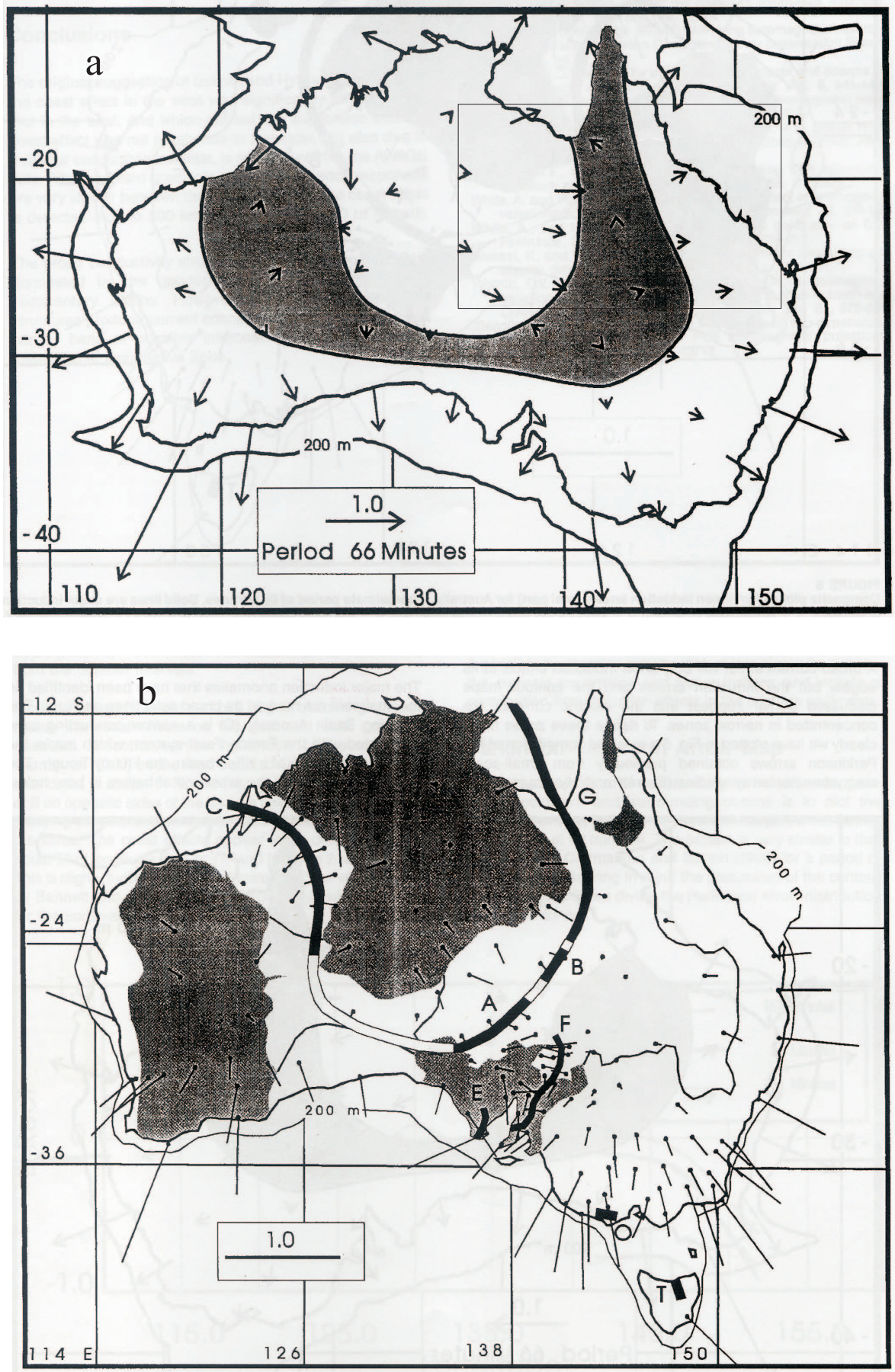

Figure 1.1: The shaded area in (a) represents the major induction zone of the Intracratonic zone as carved out by Chamalaun and Barton(1993). (b) distinguishes the conductors of the intracratonic zone and other conductors of the continent by the first letter of their names. $\mathrm{A}=$ Arckaringa Anomaly, $\mathrm{B}=$ south-west Queensland Anomaly, $\mathrm{C}=$ Canning Basin Anomaly, E=Eyre Peninsula Anomaly, $\mathrm{F}=$ Flinders Rangers Anomaly, $\mathrm{G}=$ Gulf of Carpentaria Anomaly, $\mathrm{T}=$ Taemar Anomaly and $\mathrm{O}=$ Ottway Anomaly. 
Notwithstanding, very little is known about these anomalies. Because MT and GDS coverage of western Australia is sparse, it is not yet clear if the Canning Basin Anomaly is connected to the southwest Queensland Anomaly. According to the study carried out by Woods and Lilley $(1979,1980)$, the Southwest Queensland Anomaly seemed clearly to join the Carpentaria Anomaly judging from induction arrows pattern. However, the inverted model of Wang et al. (1997) showed a break between the south-western and northwestern Queensland conductive structures. Therefore, the nature and extent of these Anomalies are still not well understood.

Chamalaun et al. (1999) motivated by Gough (1983)'s hypothesis that major conductivity anomalies may mark old plate margins, embarked on an electromagnetic survey of the Zone that hosts the Carpentaria conductivity anomaly. The hypothesis was found to be well supported by their study. Nonetheless, they did not resolve the controversy surrounding the Tasman Line. This is probably due to the method used to analyse the data- induction arrows essentially -and the limited period range used. In this thesis, we analyse not only induction arrows but also the strike direction and have modelled both horizontal and vertical perturbation tensors of the Geomagnetic Depth Sounding at longer periods to increase the resolution of conductivity contrast between boundaries in the crust and in the mantle.

Recently, similar analyses have been undertaken in some parts of Australia (Simpson (2001); Heinson and White (2005); Selway, Heinson \& Hand (2006); Hanekop (2006)). But, their focus was on the crust and mantle anisotropy. Heinson and White (2005), from their electromagnetic induction studies in the Gulf of Carpentaria, north of our study area noted that in Australia, long-wavelength patterns of long-period induction are evident from GDS arrays over many hundreds of Kilometres that reflect the relative geographical distribution of Archaen and Proterozoic cratons, Phanerozoic fold belts, major sedimentary basins, deep-crustal conducting sutures, and continent-ocean boundaries.

From long MT induction studies carried out in Palaeoproterozoic crust in central Australia, Selway, Heinson \& Hand (2006) imaged a crustal-scale conductivity contrast across a steeply-dipping boundary. But seismic reflection and gravity data previously showed this contrast that coincides with the Redbank Thrust zone as a moderately-dipping, thick skinned fault that produces one of the largest continental gravity gradients known on Earth (Selway, Heinson \& Hand (2006)). The steeply-dipping conductivity interface from MT data was interpreted by Selway and colleagues to be a strike-slip boundary separating the North Australian Craton from a younger accreted terrane. They concluded that since the steep boundary is not evident in the seismic data, it highlights the utility of MT in defining fundamental crustal boundaries. Their work is similar to our study in Queensland where we are examining the boundary between Proterozoic central Australia and the younger accreted Phanerozoic eastern Australia using EM induction.

The data analysed in this thesis were measured in 2003, 2007 and include the field campaign of 2000, Simpson (2001). The 2007 campaign was necessitated following recommendations from Magnetotellurics and GDS analysis of the 2000 and 2003 campaigns (Hanekop 2006), to extend the magnetometer arrays to the East. Measurements to the east were expected to resolve the Tasman line (Hanekop 2006). 
Chapter 2 handles the basic theory of Magnetotellurics (MT) and Geomagnetic Depth Sounding (GDS), powerful complementary techniques for investigating the electrical conductivity of the Earth. Starting from Maxwell's equations, the relevant equations that govern electromagnetic induction are presented. A detailed description of the superposition models used to interprete the data is also given in this chapter. Chapter 3 focuses on the surveyed area, its geography and tectonics. A review of previous studies is given. Chapter 4 which deals with data measurement and processing presents the MT and GDS datasets with a preliminary discussion of the conductivity features that can be decerned by looking at the processed data and discusses first implications from the data. The MT transfer functions are put into 5 regions according to the characteristics of the phase curves. Induction vectors for a selected period range are plotted, these induction vectors, especially at periods above $100 \mathrm{~s}$ show a trend that makes it easy to interprete the data. Induction vectors to the east of the array are pointing west (away from the conducting Coral Sea in the east). On the other hand, induction vectors for sites close to the Carpentaria conductity anomaly / the Tasman Line between longitude $139^{\circ}$ and $142.5^{\circ} \mathrm{E}$ are pointing clearly east for sites east of the Line and west for sites west of it. GDS perturbation tensors elements (horizontal and vertical) are presented here as contour plots for a selected number of periods. In this chapter, we also present both the MT and GDS strikes. The GDS strike directions concur well with the direction of the induction vectors; the MT strike seems consistent and free from local distortion but do not agree well with the induction vector directions.

A more detailed and rigorous interpretation of the data leading to a conductivity model of the region that will facilitate the interpretation of tectonic features and geological boundaries is given in chapter 5. Modelled and measured data are presented side by side for comparison. Geological implications of the model are meticulously discussed in the same chapter 5. Chapter 6 concludes by stating firmly that a major basement boundary (Tasman Line) that is obscured by cover and which has not been imaged by other geophysical methods can be mapped by electromagnetic methods. An appendix followed by references end the thesis.

Some of the work I describe in chapters $1,2 \& 3$ is not mine. In such cases, I have tried to reference them appropriately but rather than give an exhaustive list of references, I have confined myself to tracing ideas to the ultimate attainable source. I sincerely apologise collectively to those whose original work may not have been properly referenced. 


\section{Theory of electromagnetic methods}

Before we dive into the theory of electromagnetic induction, lets first examine briefly the source of the electromagnetic signal that is utilised in MT and GDS prospecting.

\subsection{EM signal source}

The electromagnetic fields registered by a magnetometer installed on the surface of the Earth comes from two main sources. Source signals at short periods, less than 1s (high frequencies, higher than $1 \mathrm{~Hz}$ ), originate from magnetic field disturbances trapped in the leaky wave guide formed by the Earth's surface and the ionosphere as a result of lightning discharges (Simpson and Bahr, 2005). Both the Earth's surface and the ionosphere are highly conductive compared with the atmosphere. The second source, at longer periods ( $>1 \mathrm{~s}$ ), that can be utilised in EM sounding prospecting, is due to fluctuations in the magnetic field produced by the effect of the dynamic nature of the solar wind pressure as explained in figures 2.1 and 2.2 (Kivelson and Russell, 1995). Since this source is relevant to us, as it is that which MT and GDS soundings seek to exploit, we describe it briefly. 


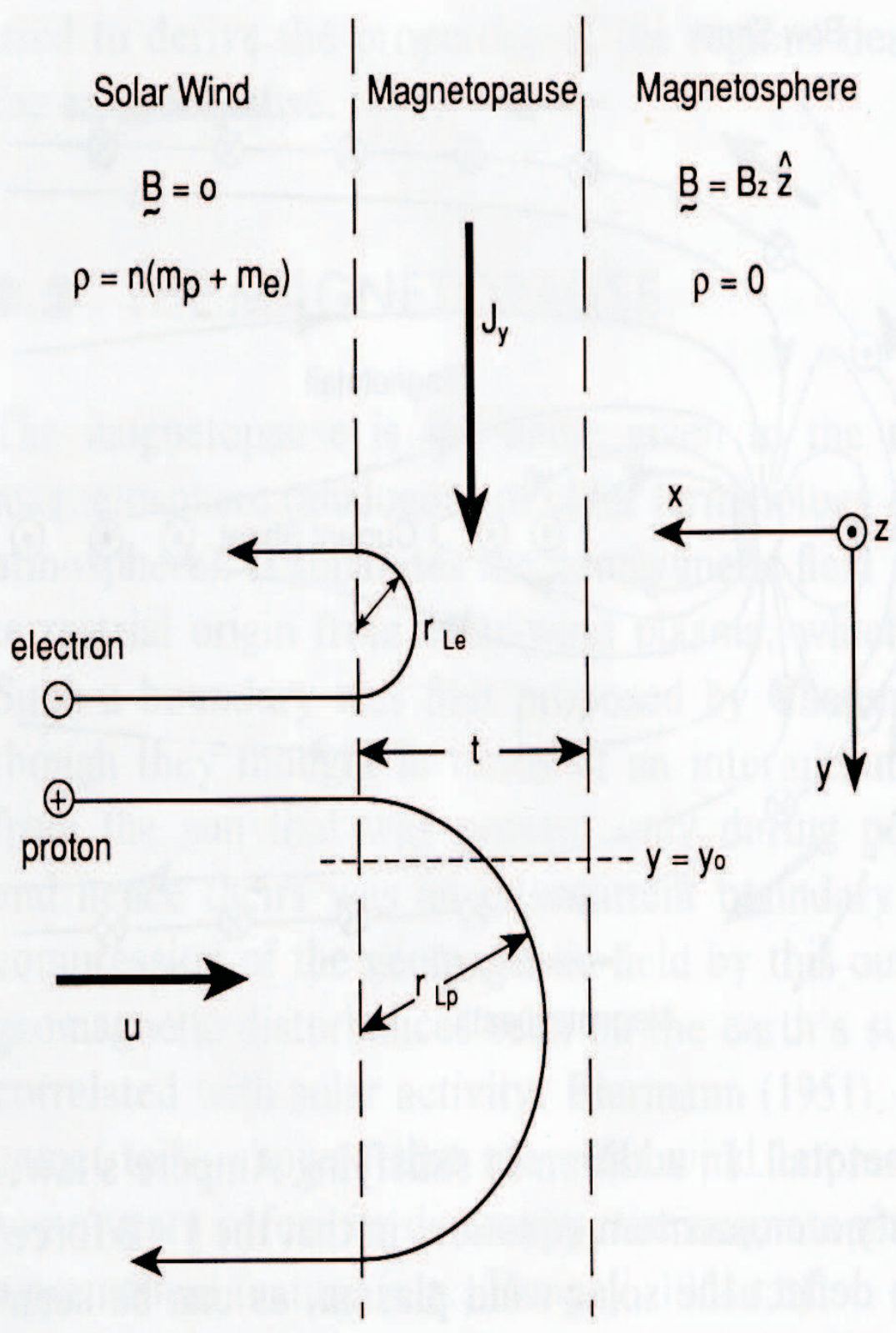

Figure 2.1: A simple illustration of magnetopause boundary separating an unmagnetized solar wind (left) from a magnetosphere containing no plasma(right). Also conspicuous from the figure is the deflection of electron and protons in opposite direction that leads to the magnetopause current. Figure modified from Kivelson \& Russell (1995) 
The solar wind (source is the sun) is a continual stream of plasma (made up of charged electrons and protons) flowing outward from the sun towards the Earth. Before reaching the magnetosphere, the net charge of the solar wind is zero. At the magnetopause (boundary of the magnetosphere), the solar wind exert a pressure on the magnetosphere and the pressure is countered by Earth's magnetic field. That is, at the magnetopause, the Earth's magnetic field acts as an obstacle to the pressure from the solar wind; on encounter of the Earth's magnetic field, the protons and electrons that constitute the plasma of the solar wind are deflected in opposite directions (charges in plasma are polarised) generating an electric field that produces a current (current is the flow of electrons and protons in opposite directions) known as magnetospause current. However, the solar wind pressure is dynamic due to fluctuations in its intensity and velocity. The fluctuations in the solar wind pressure on the magnetosphere cause the magnetopause current to also fluctuate leading to the generation of electromagnetic fluctuations (Parker, 1958) with frequencies lower than $1 \mathrm{~Hz}$ (periods longer than $1 \mathrm{~s}$ ) on the Earth's surface. The fluctuations of the magnetopause current cause a fluctuating magnetic field on the Earth's surface. These changes are called geomagnetic activity and the fluctuating waves generated are used in electromagnetic induction studies. Because the pressure of the solar wind is dynamic, the size of the magnetosphere varies with changes in the density and velocity of the solar winds. When the pressure of the solar wind increases, the magnetopause's currents increase as the magnetosphere shrinks and the magnetic field measured on the surface of the Earth rises. . 


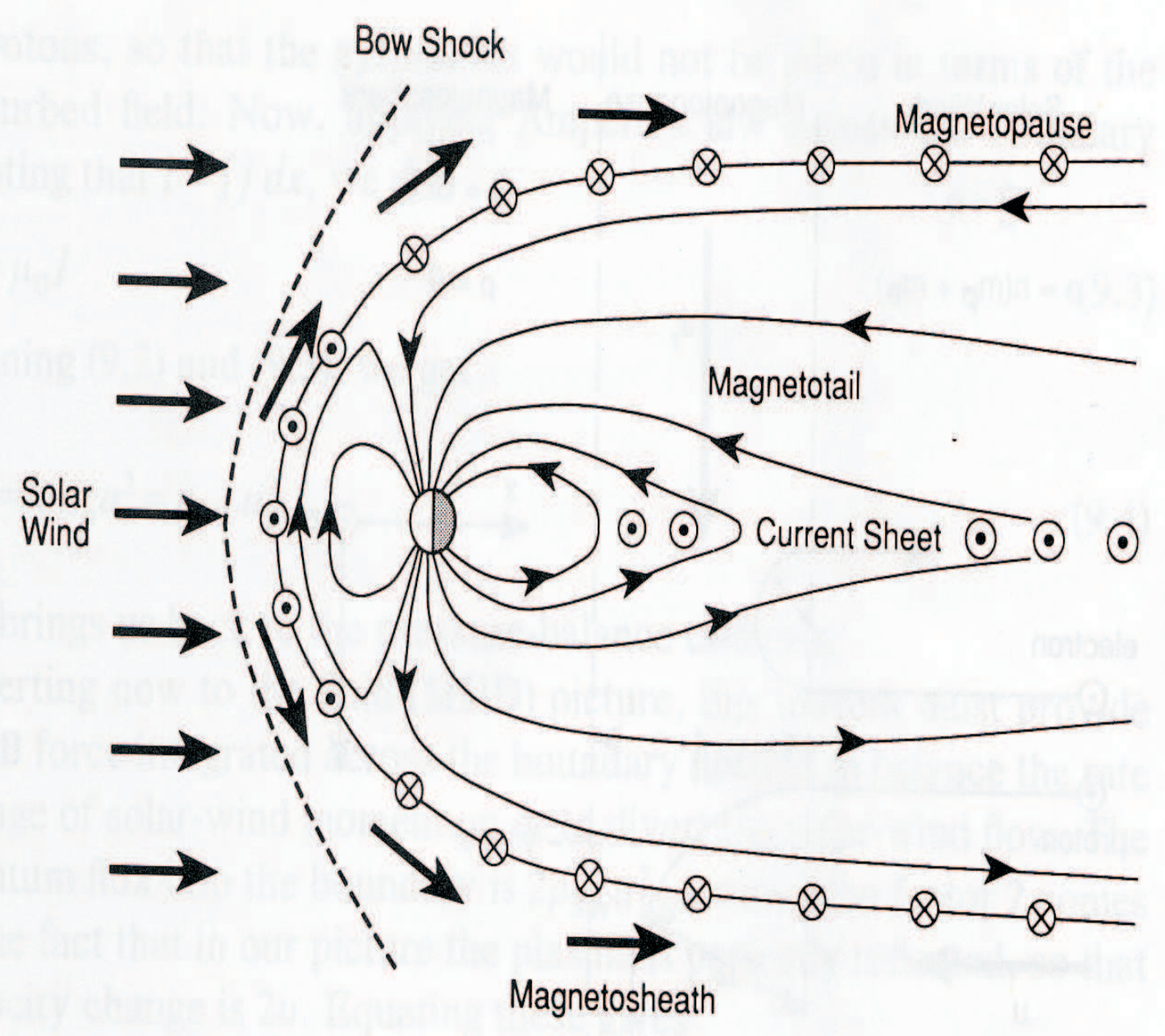

Figure 2.2: Cross section of a simple model of the magnetosphere in the noonmidnight meridian. The geomagnetic field is perfectly confined by the sheet currents flowing on the magnetopause. A second current sheet flows across the midplane of the magnetotail and joins with the magnetopause currents at the flanks of the tail. The solar wind flow is deflected at the bow shock and flows around the magnetosphere, constituting the magnetosheath. Modified figure after Kivelson \& Russell (1995) 
Figure 2.2 is a cross section of the model of the magnetosphere, again modified from Kivelson \& Russell (1995). A sudden increase in the solar wind dynamic pressure will compress the magnetosphere and move the magnetopause nearer to the Earth, and simultaneously the magnetopause current intensifies. The movement of the magnetosphere's boundary and the intensification of the current, a result of a strong and prolonged coupling of the solar wind to the magnetosphere leads to intense geomagnetic activity often called a magnetic storm that is observed on the Earth's surface as a sudden increase in the geomagnetic-field intensity of a few tens of nanotesla. The duration of a typical storm lies between 1-5 days (Kivelson \& Russell (1995), page 407). The largest geomagnetic field fluctuations (about an order of a few hundred nT) occur during magnetic storms (Simpson and Bahr, 2005), which takes place due to irregular increases in the rate at which plasma is ejected from the sun. These geomagnetic field fluctuations induce currents in the subsurface. The induced currents then diffuse downwards into the Earth and the rate of attenuation of these currents depends on the conductivity of the subsurface. The process is commonly used to probe depths of several hundred kilometres through what is known as a passive EM induction technique. The induction process is governed by the time dependent diffusion equation which we proceed to derive in the next section. But before we proceed to look at the diffusion equation, lets mention briefly the nature and effects of Sq variations.

\section{Sq variations}

As discussed above, the magnetic field observed on the Earth's surface due to the interaction of the solar wind with the main field at the magnetosphere fluctuates. Therefore at any geomagnetic observatory or station, the daily record of the geomagnetic fluctuations often shows a large number of random changes in the field that represents the superposition of many spectral components. The general increase in amplitude of the spectral components is proportional to increasing period. These spectral field variations have as origin the unique current sources in the ionosphere and magnetosphere as previously discussed under EM signal source. However, there are days that are undisturbed by solar-terrestrial and particle activity. On these days the geomagnetic records are changing smoothly especially during the daylight hours (Campbell et al., 1997). Indeed, the smooth/systematic changes depend primarily on local time and latitude (Encyclopedia Britannica Online). These slow smooth changes or variations are overshadowed by essentially 24-, 12-, 8-, and 6 -hour period spectral components (Campbell et al., 1997) in the field configuration with few of the irregularly appearing, shorter or longer period changes present. On such days, the oscillations of the three orthogonal field components $\left(H_{x}, H_{y}\right.$ and $\left.H_{z}\right)$ produce records that are anticipatively similar to others recorded many days earlier. Such records describe the "'quiet daily geomagnetic field variations"'. When the small but persistent effects attributed to the lunar tidal forces are put aside, the changes are commonly referred to as 'Sq' meaning solar quiet fields or the Sq Daily variations or Diurnal variations. The spectral lines at periods of the order of $10^{5} \mathrm{~s}$ are harmonics of Sq daily variation (Simpson and Bahr, 2005) which can be utilised in electromagnetic induction studies to probe mantle depths and beyond. 


\subsection{Maxwell's equations}

To derive the time dependent diffusion equation that governs electromagnetic phenomena, we begin from Maxwell's equations. The behaviour of electromagnetic fields at any frequency is governed by Maxwell's equations, which determine the analytical relationship in the form of a system of first order vector equations between the components of magnetic and electric fields, and the parameters of the medium ( electric conductivity $\sigma$, permittivity $\epsilon$ and magnetic permeability $\mu$ ). In simplified differential form, the equations are as follows:

$$
\begin{gathered}
\nabla \times \mathbf{H}=\mathbf{J}+\frac{\partial \mathbf{D}}{\partial t} \\
\nabla \times \mathbf{E}=-\frac{\partial \mathbf{B}}{\partial t} \\
\nabla \cdot \mathbf{B}=0 \\
\nabla \cdot \mathbf{D}=\rho_{e}
\end{gathered}
$$

where $\mathbf{E}$ is a complex vector denoting the electric field ( in $\mathbf{V} m^{-1}$ ), $\mathbf{B}$ is the magnetic induction ( in $\mathbf{T}$ ), $\mathbf{H}$ is the magnetic intensity ( in $\mathbf{A} m^{-1}$ ), $\mathbf{D}$ is electric displacement (in $\mathrm{Cm}^{-2}$ ), $\mathrm{J}$ is the current density owing to free charges (in $\mathbf{A} m^{-2}$ ) and $\rho_{e}$ is the electric charge density in (in $\mathrm{Cm}^{-3}$ ). These are the equations that are generally used in problems of electromagnetic induction in the Earth.

Equation (2.1) is Ampere's law, it denotes the electrical current (both the conductive current $\mathbf{J}$ and the displacement current $\frac{\partial \mathbf{D}}{\partial t}$ ) induced by the magnetic field. Equation (2.2) is Faraday's law; it denotes that the fluctuation of the magnetic field induces the electrical field. Equations (2.3) and (2.4) are derived from Gaussian Theorem, one for the magnetic field and the other for the electrical field respectively.

Let us assume that the region of modelling consists of an upper half-space (atmosphere), and a lower conducting half-space (Earth) characterized by an inhomogeneous distribution of conductivity. The system of equations (2.1-2.4) in the conducting medium may be modified so that equations (2.1) and (2.2) involve only the electric and magnetic fields, respectively: Assuming that displacement currents are negligible, equation (2.1) reduced to

$$
\nabla \times \mathbf{H}=\mathbf{J}
$$

For linear isotropic media;

$$
\mathbf{B}=\mu \mathbf{H}
$$

and

$$
\mathbf{D}=\epsilon \mathbf{E}
$$


Assuming $\mathbf{A}=\mathbf{A}_{o} e^{i \omega t}$ where $\mathbf{A}=\mathbf{E}$ or $\mathbf{B}$, introducing $\mathbf{J}=\sigma \mathbf{E}$ and making use of the relation $\nabla \times(\nabla \times A)=(\nabla \cdot \nabla \cdot \mathbf{A})-\nabla^{2} \mathbf{A}$, and assuming an Earth model for which $\nabla \cdot \mathbf{E}=$ 0 , equation (2.1) becomes

$$
\nabla \times \nabla \times \mathbf{E}=(\nabla \cdot \nabla \cdot \mathbf{E})-\nabla^{2} \mathbf{E}=-\nabla \times \frac{\partial \mathbf{B}}{\partial t}=\mu \sigma \frac{\partial \mathbf{E}}{\partial t}
$$

Therefore

$$
\nabla^{2} \mathbf{E}=\mu \sigma \frac{\partial \mathbf{E}}{\partial t}=-i \omega \mu \sigma \mathbf{E}
$$

In a similar manner,

$$
\nabla^{2} \mathbf{H}=\mu \sigma \frac{\partial \mathbf{H}}{\partial t}=-i \omega \mu \sigma \mathbf{H}
$$

Equations (2.9) and (2.10) are diffusion equations with and $\mathbf{E}$ or $\mathbf{H}$ being the amplitude and $\sqrt{i \omega \mu \sigma}$ the wave number. They tell us that electromagnetic depth sounding measurements utilise a source of energy that diffuses through the Earth. It is for this reason that MT and GDS measurements yield volume soundings (the response functions are volumetric averages of the sample medium) and clear cut boundaries are difficult to delineate. Equations (2.9) and (2.10) can be written in the form of the vector Helmholtz equation as follows

$$
\nabla^{2} \mathbf{A}-\gamma^{2} \mathbf{A}=0
$$

where $\mathbf{A}=\mathbf{E}$ or $\mathbf{H}$ again is the amplitude and $\gamma^{2}=-\omega \mu \sigma$; implies $\gamma$ is complex and equal to $i \omega \mu \sigma$.

If we assume a uniform plane wave propagating in the $+\mathrm{z}$ direction, with $\mathbf{A}=\mathbf{E}_{x}$, the solution of equation (2.11) takes the form:

$$
\mathbf{E}_{x}=\mathbf{E}_{o} e^{-\alpha z} e^{-i \beta z}
$$

where

$$
\gamma=\alpha+i \beta=\sqrt{i \omega \mu \sigma}=\sqrt{i} \sqrt{\omega \mu \sigma}=\frac{1+i}{\sqrt{2}} \sqrt{\omega \mu \sigma}=\sqrt{\omega \mu \sigma / 2}+i \sqrt{\omega \mu \sigma / 2} .
$$

$\alpha$ is the attenuation constant and $\beta$ is the phase constant. The inverse of $\gamma$ called the inductive scale length is also referred to as the Schmucker-Weidelt transfer function (Weidelt, 1972; Schmucker, 1973). It has dimensions of length, however, it is complex.

Equation (2.12) shows that the solution field does not freely propagate, but decays exponentially with depth z; this decay, dependent upon the conductivity and frequency, is called the "skin effect".

The skin depth $\delta$, defined as that depth at which the fields reduce to 1 /e of the surface value is obtained from the propagation constant $\gamma$ as follows: From equation (2.11),

$$
e^{-\alpha \delta}=e^{-1} \rightarrow \delta=\frac{1}{\alpha}=\frac{1}{\sqrt{\omega \mu \sigma / 2}}
$$

Therefore the inverse of $\alpha$, the real part of $\gamma$ gives the electromagnetic skin depth:

$$
\delta=\sqrt{2 / \omega \mu \sigma}
$$


Equation (2.14) tells us that the skin depth gets smaller as the conductivity increases meaning that electromagnetic waves are easily attenuated in media with enhanced conductivity. Assuming a free space value for $\mu$, the depth of penetration depends on the conductivity of the medium and the frequency of electromagnetic energy. Therefore the frequency range appropriate for MT and GDS investigations depends upon the depths of interest.

We had earlier mentioned that the conduction current term is much greater than the displacement current term for studies of the earth and the propagation constant in the ground is therefore much greater than in the air. Thus, the Earth has a high refractive index with respect to the air, and incident waves will be refracted almost straight down, regardless of the angle of incidence. The relationship between the incident and the refracted waves can be used to calculate the impedance which is key in MT analysis.

\subsection{Magnetotellurics}

For an incident horizontal wave $E$, again assuming a uniform wave propagating in the $\mathrm{z}$ direction, we have

$$
E^{I}=E_{x}^{I} e^{i(\omega t-\gamma z)}
$$

the refracted wave is obtained by matching phases at the boundary (the phase of the incident and the refracted wave is the same at the boundary), as

$$
E^{T}=E_{x}^{T} e^{i(\omega t-\gamma z)}
$$

From Maxwell equation 2.2, the associated tangential magnetic field is derived as follows

$$
\nabla \times \mathbf{E}=\frac{\partial \mathbf{E}_{x}^{T}}{\partial z}=i \omega \mu H_{y}^{T}
$$

But

$$
\frac{\partial \mathbf{E}_{x}^{T}}{\partial z}=i \gamma E_{x}^{T}=i \omega \mu H_{y}^{T}
$$

Therefore

$$
H_{y}^{T}=\frac{\gamma}{\mu \omega} E_{x}^{T}
$$

And the impedance is defined as

$$
Z=E_{x}^{T} / H_{y}^{T}=\omega \mu / \gamma
$$

where $\gamma^{2}=i \omega \mu \sigma$ as before .

In a similar way, for an incident $\mathrm{H}$ horizontal wave,

$$
H^{I}=H_{x}^{I} e^{i(\omega t-\gamma z)}
$$


and the refracted wave or is

$$
H^{T}=H_{x}^{T} e^{i(\omega t-\gamma z)}
$$

From Maxwell equation 2.1, the associated tangential electric field is

$$
\mathbf{E}_{y}^{T}=\frac{i \gamma}{\sigma} \mathbf{H}_{x}^{T}
$$

Therefore, the impedance is given as below

$$
Z=-E_{y} / H_{x}=-\frac{i \gamma}{\sigma}
$$

The above calculation shows that the impedance is independent of the source field geometry, $k_{x}+k_{y}$ and $k_{z}$.

For sources with relatively long wavelengths, the E parallel and $\mathrm{H}$ horizontal impedances are equal, and thus the impedance for a homogeneous halfspace is isotropic. This impedance is given by

$$
Z(\omega)=E_{x} / H_{y}=\frac{\mu \omega}{k}=\frac{-i k}{\sigma}=\sqrt{\frac{-i \mu \omega}{\sigma}}
$$

In most literature the impedance tensor is popularly defined in terms of $\mathrm{E}$ and $\mathrm{B}$ by the expression $\mathrm{Z}=\mu E / B$ where $\mathrm{H}=\mathrm{B} / \mu$. Therefore, the complex impedance tensor is then given by:

$$
\left(\begin{array}{c}
E_{x} \\
E_{y}
\end{array}\right)=\left(\begin{array}{cc}
0 & Z \\
-Z & 0
\end{array}\right)\left(\begin{array}{c}
B_{x} \\
B_{y}
\end{array}\right)=\underline{\underline{Z}}\left(\begin{array}{c}
B_{x} \\
B_{y}
\end{array}\right)
$$

The resistivity for the uniform Earth described above is obtained from the impedance as

$$
\rho=\frac{1}{\sigma}=\frac{1}{\mu \omega}|Z(\omega)|^{2}
$$

In this case of a uniform Earth, the calculated $\rho$ will be the true Earth resistivity.

The derivation above holds for a homogeneous half space. The general form of equation (2.24) that can be used to describe any conductivity structure is given by:

$$
\left(\begin{array}{c}
E_{x} \\
E_{y}
\end{array}\right)=\left(\begin{array}{ll}
Z_{x x} & Z_{y x} \\
Z_{x y} & Z_{y y}
\end{array}\right)\left(\begin{array}{c}
B_{x} \\
B_{y}
\end{array}\right)
$$

The resistivity that will be obtained from the impedance tensor in equation (2.26) for a heterogeneous Earth is called apparent resistivity which is a weighted average over the penetration depth of the signals and depends on frequency. The expression for this apparent resistivity is given as:

$$
\rho_{a, i j}(\omega)=\frac{1}{\mu \omega}\left|Z_{i j}(\omega)\right|^{2}
$$

Apart of apparent resistivity, magnetotelluric response is also commonly specified in terms of the impedance phase. The phase of the complex impedance is given by:

$$
\phi_{i j}=\tan ^{-1}\left(\frac{\operatorname{Im}\left(Z_{i j}(\omega)\right)}{\operatorname{Re}\left(Z_{i j}(\omega)\right)}\right)
$$


Going back to the case of a uniform half-space, equation 2.27 simplifies to:

$$
\phi=\tan ^{-1}\left(\frac{\operatorname{Im}(Z(\omega))}{\operatorname{Re}(Z(\omega))}\right)=\tan ^{-1}\left(-i \sqrt{\frac{\frac{\mu \omega}{2 \sigma}}{\frac{\mu \omega}{\sigma}}}\right)=\tan ^{-1}\left(\frac{-i}{\sqrt{2}}\right)=-45^{\circ}
$$

Equation (2.28) shows that for a homogeneous halfspace, the phase is independent of frequency and magnetic field lags the orthogonal electric field. For a non uniform halfspace, the phase becomes frequency dependent. In this case, the phase lead of the measured electric field over the measured magnetic field also provides important information on the conductivity structure. For magnetotelluric measurements made over a homogeneous half space (equation 2.28), the phase will be equal to $45^{\circ}$. For $1 \mathrm{D}$ and $2 \mathrm{D}$ structures the phase lies between $0^{\circ}$ and $90^{\circ}$, and also for most 3D structures it lies in this range. The phase response provides an indication of changes of conductivity with depth, at the penetration depth. Phase values above $45^{\circ}$ symbolize that conductivity is increasing with depth, whereas phase values less than $45^{\circ}$ give an indication that conductivity is decreasing with depth (Simpson \& Bahr, 2005; Lezata, 2001).

To better understand the interpretation of $\rho_{a}$ and $\phi$, and the period dependence of geomagnetic induction, a 1D model of the Earth consisting of two-layers is useful. For such a two layer model, one can vary the conductivity ratio between the top and bottom layer and obtain a plot of apparent resistivity and phase as a function of the period T. At short periods $\rho_{a}$ will tend to the resistivity of the upper layer; at longer periods $\rho_{a}$ will take on the resistivity values of the lower layer. This is the direct consequence of the skin-depth relation $\left(\delta=\sqrt{2 / \mu_{o} \sigma \omega}\right) ; \mu_{o}$ is the magnetic permeability of free space: longer periods tend to penetrate deeper into the Earth while shorter periods are attenuated more rapidly, and the penetration is shallower. At very short periods, the penetration is limited only to the top layer and the subsurface appears as a homogeneous halfspace leading to a $45^{\circ}$. Equally, at very long periods, the fields are completely in the second layer and the subsurface again appears as a uniform halfspace and the phase again is $45^{\circ}$. Therefore, for a two layer model, the phase at shortest and longest period is $45^{\circ}$ and that in between is a function of the ratio of the conductivities of the layers.

\subsubsection{Two dimensional problem}

An induction problem is two-dimensional (2D) if all parameters and field vectors are independent of one of the horizontal coordinates, say x. For the Earth, the conductivity varies with depth and in one horizontal direction. The direction perpendicular to the horizontal conductivity gradient is called strike direction. Let the $\mathrm{x}$-axis be the strike direction (i.e., $\left.\frac{\partial}{\partial x}=0\right)(\sigma=\sigma(y, z))$, Maxwell's equations (2.1 \& 2.2) without displacement currents can be simplified to obtain equation (2.29) below.

$$
\left(\begin{array}{c}
\frac{\partial B_{z}}{\partial y}-\frac{\partial B_{y}}{\partial z} \\
\frac{\partial B_{x}}{\partial z} \\
-\frac{\partial B_{x}}{\partial y}
\end{array}\right)=\mu \sigma\left(\begin{array}{c}
E_{x} \\
E_{y} \\
E_{z}
\end{array}\right) \text { and }\left(\begin{array}{c}
\frac{\partial E_{z}}{\partial y}-\frac{\partial E_{y}}{\partial z} \\
\frac{\partial E_{x}}{\partial z} \\
-\frac{\partial E_{x}}{\partial y}
\end{array}\right)=-i \omega\left(\begin{array}{c}
B_{x} \\
B_{y} \\
B_{z}
\end{array}\right)
$$

The above system of equations can be separated into two independent modes: one incorporating electric fields parallel to strike (E-polarization), the other incorporating magnetic fields parallel to strike (B-polarization). 


\begin{tabular}{|l|l|}
\hline E-polarization (field components $\left.E_{x}, B_{y}, B_{z}\right)$ & B-polarization (field components $B_{x}, E_{y}, E_{z}$ ) \\
\hline$\frac{\partial B_{z}}{\partial y}-\frac{\partial B_{y}}{\partial z}=\mu \sigma E_{x}$ & $\frac{\partial E_{z}}{\partial y}-\frac{\partial E_{y}}{\partial z}=-i B_{x}$ \\
\hline$\frac{\partial E_{x}}{\partial z}=-i \omega B_{y}$ & $\frac{\partial B_{x}}{\partial z}=\mu \sigma E_{y}$ \\
\hline$\frac{\partial E_{x}}{\partial y}=-i \omega B_{z}$ & $\frac{\partial B_{x}}{\partial y}=-\mu \sigma E_{z}$ \\
\hline
\end{tabular}

From the definition of the MT impedance, E- and B-polarizations will give different values of the impedance. This can be summarised in the $2 \mathrm{D}$ impedance tensor which is completely decribed by only two impedances as given below

$$
\left(\begin{array}{c}
E_{x} \\
E_{y}
\end{array}\right)=\left(\begin{array}{cc}
0 & Z_{x y} \\
Z_{y x} & 0
\end{array}\right)\left(\begin{array}{l}
B_{x} \\
B_{y}
\end{array}\right)
$$

where $Z_{x y}=E_{x} / H_{y}, Z_{y x}=E_{y} / H_{x}, Z_{y y}=E_{y} / H_{y}=Z_{x x}=E_{x} / H_{x}=0$

The vanishing main diagonal elements indicate the decoupling of the two polarizations. The tensor element $Z_{x y}$ with the electric field along strike (x) and the magnetic field across strike (y) is called E-polarization or the transverse electric (TE); the tensore element $Z_{y x}$ with the electric field across strike (y) and the magnetic field along strike (x) is called B-polarization or transverse magnetic (TM) mode. In most cases, the coordinate system in which the data is measured will not coincide with the system of electromagnetic strike. In these cases the condition that $Z_{x x}=Z_{y y}=0$ is not satisfied with measured data. The condition may also fail as a result of distortion: (i) data errors imposed on a real 1-D or $2 \mathrm{D}$ inductive response, (ii) coupling of the regional 1D or 2D inductive response with localised, small scale 3D heterogeneities) or due to 3D induction effects (Simpson and Bahr, 2005).

As earlier mentioned, if the impedance is measured at an arbitrary orientation, as it is usually the case, the data is then rotated about an angle $\alpha$ using a rotation matrix $\beta$ where

$$
\beta=\left(\begin{array}{cc}
\cos \alpha & \sin \alpha \\
-\sin \alpha & \cos \alpha
\end{array}\right)
$$

In this case, the transformed field components become: $E^{\prime}=\beta E \quad H^{\prime}=\beta H$

To transform the impedance tensor, $\underline{\underline{Z}}$ such that

$$
E^{\prime}=\underline{\underline{Z}}^{\prime} H^{\prime}
$$

then $\underline{\underline{Z}}^{\prime}$ must satisfy

$$
\underline{\underline{Z}}^{\prime}=\beta Z \beta^{T}
$$

The angle $\alpha$ is the strike direction, measured counter-clockwise from the observed coordinate system and it is routinely determined from the impedance tensor. For a 3D conductivity subsurface, the impedance will have a more complex form, but is often presented after rotation to the best-fitting $2 \mathrm{D}$ form (Bahr 1988) or rotation to an orientation that maximises some combination of $Z_{x y}$ and/ or $Z_{y x}$ (Chakridi et al., 1992). However, even after rotation to coincide with the coordinate system of measurement and that of 
the electromagnetic strike, measured data rarely have zero diagonal impedance elements in any coordinate system. This is due to galvanic distortion (discussed in section 2.3.3) of the magnetotelluric response.

Before we proceed to discuss distortion, it is instructive to discuss first the concept of induction arrows which together with impedance and phase are used in the interpretation of MT data.

\subsubsection{Induction arrows}

Vertical magnetic fields are generated by lateral conductivity gradients and boundaries and spatial variations of the ratio $H_{z} / H_{y}$ can be used to identify lateral conductivity contrasts. It is based on this idea that Parkinson (1959) and Wiese (1962) introduced a relationship between the vertical and horizontal magnetic fields known as induction arrows or tipper vector ${ }^{4}$. They are called tipper vectors because the vectors transform or tip horizontal magnetic fields into the vertical plane according to the relationship:

$$
H_{z}=\left(\begin{array}{ll}
T_{x} & T_{y}
\end{array}\right)\left(\begin{array}{l}
H_{x} \\
H_{y}
\end{array}\right)
$$

The components $T_{x}, T_{y}$ are complex functions of frequency and are commonly referred to as the 'transfer functions' (Zhang et al., 1993). They are determined from the vertical anomalous magnetic field. They are important in EM induction studies because they characterize the polarization-dependent response of an anomalous conducting structure and therefore reflect the horizontal asymmetry of the excess currents of a galvanic and induction nature arising in the subsurface due to lateral variations in the electrical conductivity. From Bio-Savart's law, the component $T_{x}$ defines a contribution of excess current flowing in the y-direction (eastward), while the component $T_{y}$ defines a contribution of excess current flowing in the x-direction (northward).

The complex-valued transfer functions when combined to form a $2 \mathrm{D}$ vector called the magnetic response function (Hobbs, 1992), can be presented on a geographic map as Induction Arrows in which the real and imaginary parts of $T_{x}$ and $T_{y}$ are combined to form real and imaginary induction arrows. The length (proportional to the lateral conductivity contrast) of each arrow is given by

$$
\left(\operatorname{Re}\left(T_{x}\right)^{2}+\operatorname{Re}\left(T_{y}\right)^{2}\right)^{\frac{1}{2}} \quad \text { and } \quad\left(\operatorname{Im}\left(T_{x}\right)^{2}+\operatorname{Im}\left(T_{y}\right)^{2}\right)^{\frac{1}{2}}
$$

for real and imaginary arrows respectively; likewise, the azimuth of the real and imaginary arrows are given by

$$
\tan ^{-1}\left(\frac{ \pm R e\left(T_{y}\right)}{ \pm \operatorname{Re}\left(T_{x}\right)}\right) \text { and } \tan ^{-1}\left(\frac{ \pm \operatorname{Im}\left(T_{y}\right)}{ \pm \operatorname{Im}\left(T_{x}\right)}\right)
$$

respectively.

The azimuth of the induction vector gives an indication of the geometry of the internal current system generating anomalous vertical fields (Banks, 1973). For a 1D regional

\footnotetext{
${ }^{4}$ Initial induction vectors by Parkinson were real
} 
conductivity distribution, the excess currents are absent implying that $T_{x}=T_{y}=0$, making the vertical component of the magnetic field zero. Therefore, induction arrows do not exist in such a case. In a 2D Earth, induction arrows are associated only with the E-polarisation (Simpson \& Bahr 2005). Thus, insulator-conductor boundaries extending through a 2D Earth give rise to induction arrows that orient perpendicular to them. For a 2D model, if the strike is along the $\mathrm{x}$-axis, $T_{x}=0$ and equation (2.33) reduces to:

$$
H_{z}=T_{y} H_{y}
$$

In this case, the magnetic response function, also known as the Wiese-Parkinson matrix contains only one component oriented across strike. In the case of a 3D asymmetric model and in coordinates aligned with the radial and tangential directions with respect to strike, the Wiese-Parkinson matrix has only one component oriented radially (Berdichevsky and Dmitriev, 2008).

Unlike Parkinson (1959, 1962), Evertt \& Hyndman (1967); Schmuker (1970) extended the transfer functions from which induction vectors are calculated to include the complex domain. Therefore, the Parkinson arrows are complex and hence have real and imaginary parts. The real part indicates the strength of the vertical magnetic field while the imaginary part illustrates the change in the field and thus the change in the real induction vector.

In the Wiese convention, the one adopted in this thesis, the real part of induction vectors points away from conducting structures which cause a geomagnetic variation anomaly and has magnitude proportional to the strength of the lateral conductivity contrast. According to Parkinson's definition, the induction arrows point towards the conducting anomaly. In both conventions the emphasis is on the real arrow. However, Chen and Fung (1985) cautioned that one cannot as a general rule fix a consistent sign convention for the imaginary 'Parkinson' arrows. They pointed to the fact that when the Parkinson arrow was modified to have real and imaginary parts, the real arrow was assumed to be proportional in magnitude to the original Parkinson arrow, but reversed in sign, in order to be consistent with previous conventions.

Lilley \& Arora (1982) worked out that if all the time-dependent functions were assumed to vary in time initially according to $\exp (i \omega t)$, then the direction of the imaginary arrow is identical to the direction of the real arrow, which is anti-parallel to the conventional Parkinson arrow $\mathbf{P}$. On the other hand, if the time-dependent data are taken to vary as $\exp (-i \omega t)$, the real arrow is also anti-parallel to $\mathbf{P}$, but that the imaginary arrow is in line with P. According to Chen and Fung (1985), the physical implication of the work of Lilley \& Arora means that imaginary arrows would always point towards regions of better electrical conductivity. They proceeded to show that, using the same initial timedependent factor, when the frequency $\omega$ of the inducing field varies, the value as well as direction of the imaginary arrow changes subsequently. They found out that when the value of $\omega$ reaches a certain characteristic value $\omega_{c}$ (corresponding to a characteristic period $T_{c}$ ), the absolute value of the imaginary arrow is zero and the imaginary arrow changes in direction as $\omega$ varies across $\omega_{c}$. Chen and Fung (1985) then went further to conclude as follows: 
1) Irrespective of the time varying factor, the imaginary Parkinson arrows can change their directions as the fluctuating frequency $\omega$ changes and stated that one cannot, as a general rule, fix a consistent sign convention for the imaginary Parkinson arrows.

2) The phase difference, and hence the imaginary component of the transfer function, flips its sign at a characteristic period $T_{c}$

Similarly, Gurk (1999) stated that in the period range of induction, the imaginary arrows are expected to be opposed to the direction of real arrows; at the period of maximum induction, manifested by the maximum amplitude of the real induction arrows, the imaginary induction arrows change their sign and direction by $180^{\circ}$. Therefore, when one chooses a sign convention, extra care must be taken in the interpretation of the direction of the imaginary arrow.

\subsubsection{Distortion and dimensionality analysis of MT transfer functions}

The distinguishing feature of MT and GDS transfer functions is ascertained essentially by the dimensionality (1D, 2D or 3D) of the conductivity structure. Notwithstanding, electric charge accumulation on near-surface heterogeneities distorts the measured magnetotelluric response leading to phase mixing (distortion of the telluric orthogonality) and or static shift (distortion of the telluric amplitudes) so that the regional conductivity structure is no longer accurately represented. In such a case the measured electric field becomes a superposition of the regional electric field and a distorted one (equation 2.37).

The process whereby local conductive bodies (small 3D structures) distort the transfer functions and make the regional delineation of the underground structures difficult is termed galvanic distortion. This is due to the fact that small localized conductivity heterogeneities alter the direction and magnitude of the electric field at the measurement site. In this case, $\nabla \cdot \mathbf{E}=0$ no longer holds as previously assumed in the derivation of the diffusion equation. If the dimensions of the heterogeneity are much smaller than the signal penetration (equation 2.14) into the surrounding material, the distortion (telluric galvanic distortion i.e. phase mixing or static shift) is independent of the frequency and will affect mainly the electric field responses. In such a case the phase is not affected since parameters that describe the distortion are real and frequency independent and hence do not have any effects on the phase variations (equation 2.27). Static shift effect causes the apparent resistivity curves to be distorted by a parallel offset and makes the actual apparent resistivity difficult to determine. Sometimes, the sounding curve may be affected at all frequencies. Galvanic distortion has been and remains an obstacle in the interpretation of magnetotelluric data. The main difficulty usually encountered in the distortion removal problem is that the equations that describe the effects of galvanic distortion on the impedance tensor are underdetermined ${ }^{5}$. This explains why it is appropriate to interpret or model MT data using phase information that is free from near surface inhomogeneities rather than using apparent resistivity information.

\section{Theoretical Background: Galvanic Distortion}

\footnotetext{
${ }^{5}$ measured parameters are less than the number of unknowns
} 
The distortion produced by near surface 3D conductivity heterogeneities on the regional electric field $\mathbf{E}_{R}$ (the field that will be observed at the surface in the absence of heterogeneity) can be expressed as:

$$
\mathbf{E}(\omega)=\mathbf{E}_{R}(\omega)+\mathbf{E}_{s}(\omega)
$$

where $\mathbf{E}$ is the observed electric field, $\mathbf{E}_{s}$ is the scattered (distorted) electric field due to the action of the regional field on the conductivity heterogeneity and $\omega$ is the angular frequency. If we assume that inductive effects are negligible and that $\mathbf{E}_{R}$ does not vary much over the lateral extent of the conductivity heterogeneity (Groom \& Bahr 1992; Chave \& Smith 1994; Caldwell et al., 2004), the scattered field $\mathbf{E}_{s}$ is approximately linearly proportional to the regional field $\mathbf{E}_{R}$ and in phase with it (Smith, 1997). Based on these assumptions, equation 2.37 can be rewritten in terms of a frequency-independent linear operator or distortion matrix $\underline{\underline{D}}$ (Smith, 1997; Caldwell et al., 2004) that transforms the regional electric field vector into the distorted field $\mathbf{E}$ observed at the surface.

$$
\mathbf{E}(\omega)=\underline{\underline{D}} \mathbf{E}_{R}(\omega)
$$

$\underline{\underline{D}}$ is a frequency independent real $2 \mathrm{D}$ tensor (also called the electric distortion matrix). It is important to note that equation (2.38) is only satisfied from some threshold period where inductive effects within these conductivity heterogeneities become negligible compared with the inductive response produced by the regional conductivity body (Bibby et al., 2005). Equation (2.38) also equivalently expressed the idea (Bahr 1988, 1991) that the observed electric field is a linear superposition of the regional field and a distorted electric field.

In cases where equation (2.38) holds, the horizontal components of the observed magnetic field $\mathbf{H}$, are (approximately) equal to the corresponding components of the regional field $\mathbf{H}_{R}$, i.e.

$$
\mathbf{H}(\omega)=\mathbf{H}_{R}(\omega)
$$

The conditions under which equations (2.38) and (2.39) are good approximations are discussed in Groom \& Bahr (1992), Singer (1992), Chave \& Smith (1994) and Utada \& Munekane (2000).

The general expression for the impedance tensor $\underline{\underline{Z_{i j}}}(\omega)$ is given by:

$$
\mathbf{E}(\omega)=\underline{\underline{Z}}(\omega) \mathbf{H}(\omega)
$$

Where we assume that $\underline{\underline{Z}}(\omega) \equiv Z_{i j}(\omega)$ and from now onwards, the two forms will be used interchangeably. The regional impedance tensor is similarly defined as

$$
\mathbf{E}_{R}(\omega)=\underline{\underline{Z}}_{R}(\omega) \mathbf{H}_{R}(\omega)
$$

Using equations 2.38-2.41, the distorted impedance is then expressed as

$$
\mathbf{E}(\omega)=\underline{\underline{D}} \mathbf{E}_{R}(\omega)=\underline{\underline{D}}\left(\underline{\underline{Z}}_{R}(\omega) \mathbf{H}_{R}(\omega)\right)=\underline{\underline{D}}\left(\underline{\underline{Z}}_{R}(\omega)\right) \mathbf{H}(\omega)
$$

and therefore, the relationship between the observed (distorted) and the regional impedance tensor is:

$$
\underline{\underline{Z}}=\underline{\underline{D}} \cdot \underline{\underline{Z}}_{R}
$$


Because $\underline{\underline{D}}$ is unknown, and the measured number of parameters in $\underline{\underline{Z}}$ are less than the unknow number of parameters in $\underline{\underline{D}} \cdot \underline{\underline{Z}}_{R}$, the amplitude information contained in $\underline{\underline{Z}}_{R}$ can only be recovered from $\underline{\underline{Z}}$ if we make some assumptions about $\underline{\underline{D}} \cdot \underline{\underline{Z}}_{R}$.

The determination of the undistorted regional impedance $\underline{\underline{Z}}_{R}$ has been a central issue (challenge)in MT studies. To address the problem, superimposition (decomposition) models (Groom and Bailey, 1989), (Bahr, 1988) were formulated in which the data are decomposed into a local, non-inductive response (galvanic distortion) owing to multi-dimensional heterogeneities with dimensions far less than the inductive scale length of the data and a regional inductive response. The core idea on which the decomposition surmise is based, whether solved using the Bahr or Groom formulation, is the requirement that the distortion tensor should be real and independent of frequency as in equation (2.38).

In the Groom \& Bailey decomposition model, the regional impedance tensor $\underline{\underline{Z}}_{R}$ is assumed to be $2 \mathrm{D}$ (shortcoming of the model) i.e. according to the model, a three-dimensional local anomaly underlain by a regional two-dimensional structure is assumed. The distortion matrix $\underline{\underline{D}}$ (equation 2.43) is then separated into three linearly independent, $2 \times 2$ matrices $\underline{\underline{T}}, \underline{\underline{S}}$, and $\underline{\underline{A}}$ and a real number $\mathrm{g}$ (Groom \& Bailey $(1989,1991))$ as follows:

$$
\underline{\underline{D}}=g \underline{\underline{T}} \cdot \underline{\underline{S}} \cdot \underline{\underline{A}}=g \cdot N_{1}\left(\begin{array}{cc}
1 & -t \\
t & 1
\end{array}\right) \cdot N_{2}\left(\begin{array}{cc}
1 & e \\
e & 1
\end{array}\right) \cdot N_{3}\left(\begin{array}{cc}
1+s & 0 \\
0 & 1-s
\end{array}\right)
$$

where $N_{1}=1 / \sqrt{1+t^{2}}, N_{2}=1 / \sqrt{1+e^{2}}$, and $N_{3}=1 / \sqrt{1+s^{2}}$, are normalisation factors and $\mathrm{g}$ is the scalar gain factor; $\underline{\underline{S}}$ the shear tensor (which provides a measure of the local polarisation of the electric field response ${ }^{6}$. The shear $\underline{\underline{S}}$ is normalised by the shear parameter $N_{2}$ (equation 2.44); $\underline{\underline{T}}$ is the twist tensor ${ }^{7}$ and it is normalised by the twist parameter $N_{1}$ in equation $(2.44) . \underline{\underline{A}}$ is the anisotropy tensor; it does not refer to electrical anisotropy, but is an effect resulting from the action of small scale $2 \mathrm{D}$ and or $3 \mathrm{D}$ scatters (distorters). In general, the factorization separates the distortion matrix into tensors that have a physical meaning in respect with the electric field involved (Groom \& Baily, 1989). One can also easily observe that the distortion or scattering tensor $\underline{\underline{D}}$ in the Groom \& Bailey $(1989,1991)$ decomposition is factored as a product of modified forms of the Pauli spin matrices multiplied by the scalar site gain g. Details for the need and function of the four independent parameters in the distortion tensor factorisation as proposed by Groom \& Bailey can be found in Simpson \& Bahr (2005).

In order to separate the localised effects of 3D current channelling from the regional 2D inductive behaviour, the impedance tensor problem is factorised in terms of a rotation matrix, $\underline{\underline{\beta_{\alpha}}}$ and the distortion tensor $\underline{\underline{D}}$ (equation 2.44) above. Therefore, making use of the factorization of the distortion matrix, in the coordinate system of the $2 \mathrm{D}$ regional structure, equation (2.43) is now expressed as:

$$
\underline{\underline{Z}}=(g \underline{\underline{T}} \cdot \underline{\underline{S}} \cdot \underline{\underline{A}}) \underline{\underline{Z}}_{R}
$$

\footnotetext{
${ }^{6}$ the physical meaning of the shear is that it represents the deflection of the electric field by an angle $\beta_{e}$, clockwise with respect to the horizontal $\mathrm{x}$-axis and counter-clockwise for the other horizontal axis

${ }^{7}$ The twist parameter $\mathrm{t}=\tan \beta_{t}$ provides a measure of the electric field rotation through a clockwise angle $\beta_{t}$ due to additional anomalous DC-currents
} 
For an arbitrary coordinate system, e.g. the measured coordinate system, the observed impedance tensor $\underline{\underline{Z}}$ is expressed as:

$$
\underline{\underline{Z}}=\underline{\underline{\beta}}_{\alpha}(g \underline{\underline{T}} \cdot \underline{\underline{S}} \cdot \underline{\underline{A}}) \underline{\underline{Z}}_{R}^{\prime} \underline{\underline{\beta}}_{\alpha}^{T}
$$

where $\underline{\underline{Z}}_{R}^{\prime}$ is a 2D (anti-diagonal) impedance tensor and $\alpha$ is the (unknown) strike of the conductivity structure. Equation (2.46) has a total of nine unknowns $\alpha$ : (the regional strike angle), four elements of the 2D regional impedance tensor (two complex regional impedances) and the 4 components of the factorised distortion tensor. $\underline{\underline{Z}}$ has only eight measured quantities, hence equation (2.46) is underdetermined (has no unique solution). The unknown parameters can only be determined from the observed impedance tensor if some assumptions are made. Groom and Bailey (1989b) showed that the anisotropy A and the gain factor $\mathrm{g}$ cannot be determined but can be incorporated into an equivalent 2 D impedance tensor that only differ from $\underline{\underline{Z}}_{R}^{\prime}$ in that it is scaled by a real, frequencyindependent factor:

$$
\underline{\underline{Z}}_{R}^{\prime}=\underline{\underline{Z}}_{2 D}^{R} g \underline{\underline{A}}
$$

The shapes of the apparent resistivity and impedance phase curves remain unchanged by this transformation, however, the apparent resistivity curves is shifted by an unknown scaling factor (Simpson \& Bahr, 2005). The physical meaning of the product $g \underline{\underline{A}}$ is the static shift factor which cannot be determined by the tensor decomposition. The above transformation reduces the number of unknowns by two; the seven unknown parameters and the regional phase can then be determined uniquely at each period from what is now an overdetermined problem (Caldwell et al., 2004). The goal is to determine the strike direction of the regional 2D structure, once the twist and shear parameters have been estimated. To do this, one proceeds as follows. First using equation (2.47), equation (2.46) can be expressed as:

$$
\underline{\underline{Z}}=\underline{\underline{\beta}}_{\alpha}\left(\begin{array}{cc}
1-t e & e-t \\
e+t & 1+t
\end{array}\right)\left(\begin{array}{cc}
0 & Z_{x y}^{R} \\
Z_{y x}^{R} & o
\end{array}\right) \underline{\underline{\beta}}_{\alpha}^{T}
$$

The seven parameters to be solved are $\operatorname{Re}\left(Z_{x y}^{R}\right), \operatorname{Im}\left(Z_{x y}^{R}\right) ; \operatorname{Re}\left(Z_{y x}^{R}\right), \operatorname{Im}\left(Z_{y x}^{R}\right), \mathrm{t}$, e and $\alpha$ where $\alpha$ represents the strike angle, $\mathrm{t}$ and e the distortion parameters twist and shear repectively.

The observed impedance tensor, $\underline{\underline{Z}}$ with 8 known parameters can be expressed in terms of the Pauli spin matrices (Jones \& Groom, 1993; Lezaeta, 2001):

$\epsilon_{1}=\left(\begin{array}{ll}0 & 1 \\ 1 & 0\end{array}\right), \epsilon_{2}=\left(\begin{array}{cc}0 & -1 \\ 1 & 0\end{array}\right), \epsilon_{3}=\left(\begin{array}{cc}1 & 0 \\ 0 & -1\end{array}\right)$

and

$\underline{\underline{Z}}=\frac{1}{2}\left(a_{0} \mathbf{I}+a_{1} \epsilon_{1}+a_{2} \epsilon_{2}+a_{3} \epsilon_{3}\right)$

where

$a_{0}=Z_{x x}+Z_{y y}, a_{1}=Z_{x y}+Z_{y x}, a_{2}=Z_{x y}-Z_{y x}, a_{3}=Z_{x x}-Z_{y y}$. 
The above lead to the following system of non-linear equations with seven unknowns (Lezaeta, 2001):

$$
\begin{aligned}
& a_{0}=t\left(Z_{x y}^{R}+Z_{y x}^{R}\right)+e\left(Z_{x y}^{R}-Z_{y x}^{R}\right) \\
& a_{1}=\left[(1-e t) Z_{x y}^{R}-(1+e t) Z_{y x}^{R}\right] \cos 2 \alpha-\left[(e+t) Z_{x y}^{R}+(e-t)\right] \sin 2 \alpha \\
& a_{2}=-(1-e t) Z_{x y}^{R}-(1+e t) Z_{y x}^{R} \\
& a_{3}=-\left[(1-e t) Z_{x y}^{R}-(1+e t) Z_{y x}^{R}\right] \cos 2 \alpha-\left[(e+t) Z_{x y}^{R}+(e-t)\right] \sin 2 \alpha
\end{aligned}
$$

These equations can be used to solve for the distortion parameters $\mathrm{t}$ and e, the strike $\alpha$ and the regional impedances can be estimated using a least square method.

Although in theory, Groom and Bailey (1989) obtained a unique solution for their decomposition model, measured data which is contaminated with noise and departures from the model, will hardly yield an exact fit in any coordinate frame. As a consequence, in the Groom-Bailey decomposition, a misfit parameter between the observed data, $\underline{\underline{Z}}_{i j}$, data errors, $\delta_{i j}$, and modelled data according to a 2 D hypothesis, $\underline{\underline{Z}}_{i j}^{m}$ is suggested as:

$$
\chi^{2}=\frac{1}{4} \frac{\sum_{j=1}^{2} \sum_{i=1}^{2}\left|Z_{i j}^{m}-Z_{i j}\right|^{2}}{\sum_{j=1}^{2} \sum_{i=1}^{2}\left|\delta_{i j}\right|^{2}}
$$

3D induction effects can only be deduced from the decomposition parameterisation by investigating distortion parameters and their misfit parameters. If 3D induction is present, the calculated distortion parameters will manifest or display frequency dependence (Simpson \& Bahr, 2005), and misfit parameters may be too large. One should also note that the misfit depends on the errors in the data. Hence the misfit may be influenced by a changing error structure in different period ranges. As a result care must be taken in interpreting the misfit. If $\chi^{2} \approx 1$, then an acceptable tensor decomposition fit with the telluric galvanic model is implied (Lezaeta, 2001).

An earlier model that has been used to resolve the regional strike and hence interpret subsurface conductivity structures with or without the presence of distortion is the phase sensitive decomposition model by Bahr (Bahr, 1988). Bahr first showed that the strike direction of a $2 \mathrm{D}$ conductivity distribution can be obtained directly from the measured impedance tensor without decomposition, i.e. without attempting to recover information about the structure of the distorted tensor. In the Bahr's method, an appropriate strike angle can be determine based on the analytical rotation properties of the observed impedance tensor. He went further to formulate a decomposition model which although was still limited by the assumption of a regional $2 \mathrm{D}$, but the model is a more complete superimposition (decomposition) model that addresses the limitations of the Swift model (Swift, 1967) and the Larsen model (Larsen, 1975). Bahr (1988) demonstrated that when the measured impedance tensor is rotated into the right strike coordinate system, the phases of the two elements in each column of the rotated impedance tensor are equal. The model addresses the difficulty that there exists a large class of measured impedances having both none vanishing diagonal components and two different phases and therefore consent with neither the Swift nor Larsen model. However, the Bahr model has ambiguity in dimensionality analyses as pointed out by Marti et al. (2005). We will discuss dimensionality in the next section. 
In the Bahr model, multi-dimensional heterogeneities with dimensions significantly less than the inductive scale length (complex length that descibes the spatial extent of fields induced in the Earth, section 2.2) of of the data are superposed on a regional 2D structure. That means a $3 \mathrm{D}$ structure is superimposed on a 2D architecture and data are separated into a non-inductive (galvanic) and an inductive response. Assuming that the data is measured in the coordinate system of the regional strike, the distorted impedance tensor $\underline{\underline{Z}}_{2 D}^{\text {dis }}$ can be expanded as

$$
\underline{\underline{Z}}_{2 D}^{\text {dis }}=\underline{\underline{D}} \cdot \underline{\underline{Z}}_{2 D}=\left(\begin{array}{ll}
d_{11} & d_{12} \\
d_{21} & d_{22}
\end{array}\right)\left(\begin{array}{cc}
0 & Z_{x y} \\
Z_{y x} & 0
\end{array}\right)=\left(\begin{array}{ll}
d_{12} Z_{y x} & d_{11} Z_{x y} \\
d_{22} Z_{y x} & d_{21} Z_{x y}
\end{array}\right) .
$$

Since the distortion tensor elements $d_{i j}$ are real and frequency independent(only the amplitude of the electric field is affected by localised currents), within each column of $\underline{Z}_{2 D}^{\text {dis }}$, only one phase occurs as exlained earlier. In an arbitrary coordinate system, the phases of the regional impedances $Z_{x y}^{\prime}$ and $Z_{y x}^{\prime}$ will mix, because in this case, the impedance tensor elements are linear combinations of $Z_{x y}^{\prime}$ and $Z_{y x}^{\prime}$. In an arbitrary coordinate frame, the expression below is satisfied:

$$
\underline{\underline{Z}}_{2 D}^{\text {dis }}=\underline{\underline{\beta}}_{\alpha} \cdot \underline{\underline{D}} \cdot \underline{\underline{Z}}_{2 D}^{\prime} \cdot \underline{\beta}_{\alpha}^{T}
$$

If we apply the condition that the phase for the tensor elements in the columns of the impedance tensor is the same in the coordinate system of the regional strike to equation (2.51), we get:

$$
\frac{\operatorname{Re}\left[\left(Z_{2 D}^{d i s}\right)_{x x}\right]}{\operatorname{Re}\left[\left(Z_{2 D}^{d i s}\right)_{y x}\right]}=\frac{\operatorname{Im}\left[\left(Z_{2 D}^{d i s}\right)_{x x}\right]}{\operatorname{Im}\left[\left(Z_{2 D}^{\text {dis }}\right)_{y x}\right]}
$$

Expanding equation (2.51) and solving it simultaneously with equation (2.52) leads to an expression of the rotation (phase sensitive rotation) angle $\alpha$ (Bahr, 1988):

$$
\alpha=\frac{1}{2} \arctan \left(\frac{\left[S_{1}, S_{2}\right]-\left[D_{1}, D_{2}\right]}{\left[S_{1}, D 1\right]+\left[S_{2}, D_{2}\right]}\right)
$$

where $S_{1}=Z_{x x}+Z_{y y}, S_{2}=Z_{x y}+Z_{y x}, D_{1}=Z_{x x}-Z_{y y}, D_{2}=Z_{x y}-Z_{y x}$ and the commutator $[S, D]=\operatorname{Re}[S] \operatorname{Im}[D]-\operatorname{Im}[S] \operatorname{Re}[D]$.

In the situation where a rotation angle cannot be found that will satisfy the condition that the phases of the two elements in each column of the rotated impedance tensor are equal, Bahr (1991) suggested the minimisation of the phase difference $\delta$ between the elements of a given column, known as the delta technique, such that equation (2.51) becomes:

$$
\underline{\underline{Z}}_{2 D}^{\text {dis }}=\underline{\beta}_{\alpha}\left(\begin{array}{cc}
d_{12} Z_{y x}^{\prime} e^{i \delta} & d_{11} Z_{x y}^{\prime} \\
d_{22} Z_{y x}^{\prime} & d_{21} Z_{x y}^{\prime} e^{-i \delta}
\end{array}\right) \underline{\beta}_{\alpha}^{T}
$$

Similar to Swift's skew (Swift, 1967) elucidated in section 2.3.5, Bahr (1988) introduced the phase sensitive skew $\eta$ :

$$
\eta=\frac{\sqrt{\left|\left[D_{1}, S_{2}\right]-\left[S_{1}, D_{2}\right]\right|}}{\left|D_{2}\right|}
$$


a parameter which gives a measure of the extent to which an impedance tensor can be described by equations (2.51) or (2.54).

Bahr interpreted $\eta$ in a similar way to the Swift's skew, for $\eta<0.1$, equation (2.51) is the appropriate model, whereas for $0.1<\eta<0.3$ equation (2.54) might be more suitable.

Bahr's decomposition model and others (Groom and Bailey, 1989; Smith, 1995) are based on the assumption that the underlying conductivity structure is $2 \mathrm{D}$. In recent papers, Caldwell et al. (2002, 2004) and Bibby et al. (2005), used an innovative definition of a real $2 \times 2$ 'phase tensor' (which is independent of any real (galvanic) distortion that may be present) and demonstrated that the regional phase information can be recovered directly from the observed impedance tensor where both the near-surface heterogeneity and regional conductivity structures are 3D. That is, they introduced a new and an elegant method for analysing galvanically distorted MT data in a regional conductivity structure of any dimension (no assumption about the dimensionality of the underlying conductivity distribution). The phase tensor was expressed as a sum of three matrices which are clearly associated with 1D, 2D and 3D conductivity structures respectively, and the relative magnitude of their multiplying factors indicate the nature of the regional structure. Equation (24) of Weaver, Agarwal \& Lilley (2006) is an expression of the phase tensor in terms of three matrices. The first, second and third terms of the expression are regarded as the 1D, $2 \mathrm{D}$ and $3 \mathrm{D}$ contributions to the phase tensor repectively. If data were completely noise free, the vanishing of the first and second term of equation (24) would imply a 1D structure. Nonetheless, since real data is rarely free from distortion, a regional 1D structure will be implied if the second and third terms in the expression of the phase tensor are negligibly small compared with the first term. The regional structure was interpreted as two dimensional in the case where the third term is zero or insignificant compared with the first two terms. For details, refer to Weaver, Agarwal \& Lilley (2000, 2006). However, their method could not actually distinguish between galvanically distorted and undistorted data in 1D and 2D regions. Weaver et al, (2006) swayed by the ideas of Caldwell et al. (2004), argued that the distinction is ultimately not important and unnecessary with real data, because real data are never free of noise and are distorted in some sense. They pointed out that strictly, 2D or 1D configuration are only found in idealised mathematical models, not in the real world.

\subsubsection{Magnetic distortion}

In section 2.3.3, we had stated that all decomposition schemes take into account only the local distortion of electric fields due to the accumulation of electric charge along near surface inhomogeneities. Therefore, the anomalous magnetic field associated with the disturbed current flow is regarded as being too small to give rise to any significant distortion of the magnetic field. Nonetheless the effect of magnetic distortion can be found at relatively short periods because the term $\underline{\underline{C}} \cdot \underline{\underline{Z}}_{R}$ in equation (2.59) will become smaller for long periods.

But in general, there is still an on going argument concerning the magnitude of magnetic distortion and its contribution to the measured impedance tensor resulting from the galvanic distortion of surface electric currents. Smith (1997) after the investigation 
of magnetic distortion and its effect on the observed impedance tensor, concluded that the 'galvanic' magnetic fields associated with the galvanic distortion of surface electric currents are proportional to the strength of currents in the surface layer. Further more, he extended the conclusion to imply that at long periods, the superficial currents become negligible and the associated magnetic fields of 'galvanic' distortion become equally insignificant as mentioned above. Therefore at periods where a considerable proportion of the induced currents are affected by the distorting heterogenieties, we should expect the associated magnetic field to be significant.

Chave \& Smith (1994) developed a theoretical technique of the galvanic-distortion of magnetic and electric fields, and treated the problem of evaluating their effects in measured impedance tensor. Their theoretical treatment showed that some parameters associated with magnetic fields of galvanically distorted electric currents cannot be recovered from an observed magnetotelluric impedance tensor and the other parameters can be recovered by solving a system of non-linear equations using a general linearized method. Smith (1997) followed the approach adopted by Chave \& Smith and derived a simpler system of equations which has an analytic solution for data at a single frequency. For multiple frequencies, he demonstrated that the system can be solved using a simple iterative method. In the end, it was concluded that magnetic distortion may be significant at higher frequencies (short periods) as mentioned above. Agawal \& Weaver (2000), in an approach similar to that used by Smith, made a detailed investigation of magnetic distortion and concluded that the inclusion of magnetic distortion in the decomposition of the MT impedance tensor elements would in general provide only triffling improvement even for reasonable cases of strong current channelling on land observations, over the conventional decomposition methods in which only distortion of the electric field is considered.

\section{Theory of Magnetic Distortion}

From section 2.3.3, the measured electric field is given by equation (2.37). Since the magnetic fields, $H_{g}$ of the galvanically distorted currents are proportional and in phase with the currents (Smith 1997), which are proportional to and in phase with the regional electric fields, Smith (1997) expressed them as

$$
H_{g}=\underline{\underline{C}} \mathbf{E}_{R}
$$

where the distorted magnetic field tensor $\underline{\underline{C}}$ (similar to the distorted electric field tensor) is real and frequency independent. As it was treated for the electric field, the observed magnetic field can be similarly separated into its regional and galvanic parts, the equivalent equation to (2.37) for the horizontal magnetic field at the surface of the Earth is given by

$$
\mathbf{H}=\mathbf{H}_{R}+H_{g}=\mathbf{H}_{R}+\underline{\underline{C}} \mathbf{E}_{R}
$$

Equation (2.57) holds if the anomaly (distorter) is small enough (Ogawa, 2002). Recalling that the measured MT tensor is defined by equation (2.40) and making use of equation (2.57), we immediately obtain

$$
\underline{\underline{D}} \cdot E_{R}=\underline{\underline{Z}}\left(\mathbf{H}_{R}+\underline{\underline{C}} \mathbf{E}_{R}\right)
$$

The regional impedance tensor was given by equation (2.41), thus it is readily deduced from 2.58 that

$$
\underline{\underline{Z}}=\underline{\underline{D}} \cdot \underline{\underline{Z}} \underline{\underline{Z}}-\underline{\underline{Z}}(\underline{\underline{C}} \cdot \underline{\underline{Z}} \underline{\underline{Z}})
$$


Equation (2.59) (the tensor decomposition model) is an underdetermined problem. Its solution is given in appendix A. A detailed analytical solution and subsequent computation of tensor elements can be found in Smith (1997) and Agarwal \& Weaver (2000). If there is no magnetic distortion or if magnetic distortion is negligible, that is if $\underline{\underline{C}}=0$, then equation (2.59) reduces to the conventional equation

$\underline{\underline{Z}}=\underline{\underline{D}} \cdot \underline{\underline{Z}} \underline{\underline{Z}}$

relating the measured and regional MT tensors when electric distortion alone is present.

\subsubsection{Dimensionality analysis}

Before MT data is analysed, it is important to determine the dimensionality of the regional conductivity structure. This has been one of the main challenges of MT investigations. Several methods exist to perform dimensionality analysis of the measured data using rotational invariants of the impedance tensor. Bahr (1991) was the first author who presented a classification of the types of distortion that affect the underlying conductivity structure. He quantified the values of four rotational invariant parameters and used these parameters to classify the dimension of conductivity structures. After Bahr's pioneering and insightful work, Weaver et al. (2000, 2003, 2006), realising that the four invariant parameters of the Bahr method are insufficient to completely characterize dimensionality, defined more rotational invariant parameters (a set of seven rotational invariant parameters that are closely related to the phase tensor analysis of Caldwell et al. (2004)) which they used to characterize dimensionality. For details, see Weaver et al. (2000, 2003, 2006). Marti et al. (2005) attempted to combine the Bahr method and that of Weaver to give a more refined method. In recent papers, (as discussed under distortion) Caldwell et al. (2004) and Bibby et al. (2005) expressed the phase tensor as a sum of three matrices; each matrix representing 1D, 2D and 3D structures. Weaver et al. (2006), in a manner similar to that of Caldwell and Bibby, clearly expressed the phase tensor as a linear combination of 3 matrices. If the structure is 1D, the coefficients of the second and 3rd matrix vanish. If it is $2 \mathrm{D}$, only the coefficient of the $3 \mathrm{rd}$ matrix will vanish. A recent and intriguing paper on the topic is presented by Lilley \& Weaver(2009).

In this work, since the main objective is not dimensionality analysis, we will use Bahr's phase sensitive skew parameter to infer dimensionality.

\section{Skew parameters of dimensionality}

In the telluric galvanic model (equation 2.43) the impedance phases of each column element pair are equal since $\underline{\underline{D}}$ is real. This is as a result of the in-phase condition accomplished between the regional and the distorted electric fields. If anomalous magnetic fields are insignificant, then the 2D superposition model of equation (2.59) describes the recorded impedance. In the presence of magnetic effects, a phase difference between the elements of each column of the impedance tensor is expected contrary to Bahr's hypothesis. In this regard, Bahr (1988) proposed a rotational invariant parameter of the impedance tensor which is a measure of the level of deviation in the phases in each column, known as the 
phase sensitive skew $(\eta)$, equation (2.55) which he expressed explicitly as:

$$
\eta=\frac{\sqrt{2\left|\operatorname{Re}\left(Z_{x x}\right) \operatorname{Im}\left(Z_{y x}\right)-\operatorname{Re}\left(Z_{x y}\right) \operatorname{Im}\left(Z_{x y}\right)+\operatorname{Re}\left(Z_{x y}\right) \operatorname{Im}\left(Z_{y y}\right)-\operatorname{Re}\left(Z_{y x}\right) \operatorname{Im}\left(Z_{x x}\right)\right|}}{\left|Z_{x y}-Z_{y x}\right|}
$$

If a skew value of zero is obtained, the validity of the telluric distortion hypothesis is fulfilled, that is, a perfect regional $2 \mathrm{D}$ model can be identified. Larger values mean a deviation from this assumption. Bahr proposed a limit of 0.3 to test the validity of the galvanic model, where small phase differences due to the galvanic magnetic effects could take place. Values greater than this limit could reflect the non-validity of the galvanic magnetic distortion, meaning the presence of 3D inductive structures. However, the phase sensitive skew must be interpreted with care; if noise is added to the tensor elements, the skew values can become significantly biased leading to a false interpretation of dimensionality. Lezaeta (2001) has proposed how to bypass this problem through the estimation of the probability function of skew. She considered the tensor elements with errors to be analogous to random variables and instead of the skew value itself; its probability threshold was determined to provide information on dimensionality.

Prior to Bahr's skew, Swift (1967) had introduced a non-dimensional rotational invariant parameter called the Swift skew s which gives a measure of the amount of departure from an ideal 2D model. The skew parameter is a function of the elements of the impedance tensor; it is used to determine dimensionality and to estimate telluric distortions (produced by shallow $3 \mathrm{D}$ anomalies that cover a regional 2D structure (i.e. a superposition 2D model)) of the impedance tensor. Swift skew is defined in terms of the ratio of two rotational invariant parameters as :

$$
s=\frac{\left|Z_{x x}+Z_{y y}\right|}{\left|Z_{x y}-Z_{y x}\right|}
$$

If $\mathrm{s}$ is zero, an ideal $1 \mathrm{D}$ or 2D model is assumed. Values greater than 0.1 can be associated with a $3 \mathrm{D}$ model or a galvanic $2 \mathrm{D}$ model.

\subsection{Geomagnetic depth sounding}

Nonuniformities in the electrical conductivity of the Earth lead to the spatial deflection of regional currents through or around a local anomaly. This results to the generation of a local anomalous magnetic field $\mathbf{B}_{a}$ that is superimposed on the regional magnetic field $\mathbf{B}_{n}$. The field $\mathbf{B}$ that is observed at a local site is therefore the sum of the regional field $\mathbf{B}_{r}$ and the anomalous field. The anomalous field repesents a potential field of exclusively internal origin, in contrast to the normal field of external and internal origin (Schmucker, 2003). The geomagnetic depth sounding (GDS) method involves the separation of the time-varying magnetic fields of external origin, from those associated with induced currents flowing within the Earth (of internal origin). For locations in mid-latitudes, where the source fields are regionally homogeneous, the vertical component of magnetic field variations is due entirely to the internal currents. A 2D conductivity structure will preferentially cause a vertical magnetic field when excited by current flow in the strike direction (Bailey and Edwards, 1976). Thus, a resultant anomalous vertical fields indicate the presence of lateral conductivity variations (e.g. Parkinson, 1983). That is, lateral conductivity 
contrast distorts the local magnetic field and produce distinct anomalies in the magnetic field components (Schmucker \& Weidelt, 1975). An analysis of the relationship between the vertical and horizontal components of the time changing magnetic field gives vital information on the internal electrical conductivity structure which is used to map out local anomaly. Basic ideas about GDS are encapsulated in the influential paper by Schmucker (1970).

In contrast to MT that relates the Fourier transforms of the horizontal components of the electric field $\mathbf{E}$ and the magnetic field $\mathbf{B}$ at one site, the GDS method connects the Fourier transforms of the magnetic field $\mathbf{B}$ in all components of any site with the horizontal magnetic field of a reference site, which is expected to be located above a 1D subsurface. To be more explicit, in GDS analysis three orthogonal magnetic components are used as opposed to MT where two horizontal magnetic and electric components are used.

In GDS analysis, since the reference site is required to be $1 \mathrm{D}$, the vertical field at the reference site is zero and therefore only the horizontal $\mathbf{B}$ fields are present. The connection between the magnetic field at a measurement site with that at a reference site was first exploited by Schmucker (1970). According to Schmucker, the measured magnetic field can be separated into the normal and anomalous parts $\left(\mathbf{B}=\mathbf{B}_{n}+\mathbf{B}_{a}\right)$. The difference between the measured field $\mathbf{B}$ and normal $\mathbf{B}_{n}$ gives the anomalous field $\mathbf{B}_{a}\left(\mathbf{B}-\mathbf{B}_{n}=\mathbf{B}_{a}\right)$ which arises as a result of lateral conductivity contrast. Schmucker (1970) defined the connection between the anomalous field at an arbitrary site and a reference site by introducing what is now commonly called the Schmucker's perturbation tensor $\underline{\underline{W}}$ ( $\underline{\underline{W}}$ GDS is analogous to the impedance tensor $\underline{Z}$ in MT analysis) which maps the normal field into the anomalous field as shown below:

$$
\left(\begin{array}{c}
B_{a x} \\
B_{a y} \\
B_{a z}
\end{array}\right)=\underline{\underline{W}}\left(\begin{array}{c}
B_{n x} \\
B_{n y}
\end{array}\right)=\left(\begin{array}{cc}
h_{H} & h_{D} \\
d_{H} & d_{D} \\
z_{H} & z_{D}
\end{array}\right)\left(\begin{array}{c}
B_{n x} \\
B_{n y}
\end{array}\right)
$$

The elements of the perturbation tensor $\underline{\underline{W}}$ are dimensionless, complex variables that can be estimated in the frequency domain from field data. To determine $\underline{\underline{W}}$, data from two stations (the local site which is the site of interest and a reference site above a 1D Earth) recorded simultaneously is used. From equation (2.62), a linear relationship exists between the horizontal and vertical components of the magnetic induction, pioneered by Schmucker (1970) and reiterated by Banks (1979) and Weaver and Agarwal (1991)

$$
B_{a z}=z_{H} B_{n x}+z_{D} B_{n y}
$$

where $z_{H}$ and $z_{D}$ are complex functions of frequency and are commonly referred to as 'transfer functions' or 'Tipper' functions (Zhang et al., 1993) and characterize the polarization-dependent response of an anomalous conducting structure. The above equation with notations commonly used in GDS is the same as equation (2.33).

Earlier electromagnetic conductivity studies considered only anomalies in the vertical component, Z, in the analysis of conductivity gradients; equation (2.63) was the commonly used expression in GDS analysis. But later on, many researchers in the field of electromagnetic depth sounding (GDS) have considered explicitly the anomalies in all three components $\left(B_{x}, B_{y}\right.$ and $\left.B_{z}\right)$. Therefore nowadays the GDS method is based on geomagnetic variation anomalies characterized by anomalous vertical as well as horizontal fields. 
A discernible advantage in using anomaly in the horizontal component $(\mathrm{H})$ to delineate conductors is that, over the centre of conductors, the anomalous field $B_{a}$ has a maximum and falls rapidly to zero as one moves away from it. By contrast the anomalous vertical perturbation tensor $z_{H}$ and $z_{D}$ are zero immediately above a conductor attaining maximal values at the edges of the conductor. In this thesis, we consider anomalies in all components (vertical and horizontal).

Similar to the impedance tensor $\underline{\underline{Z}}$, the perturbation tensor $\underline{W}$ can be used to determine dimensionality of the underlying conductivity structure. If the underlying conductivity structure at the site of interest is $1 \mathrm{D}$ as is the case of the reference site, no anomalous magnetic field will exist. This makes the left hand site of equation (2.62) zero and by implication, all elements of the perturbation tensor $\underline{\underline{W}}$ will be zero. In the case of an underlying conductivity structure that is $2 \mathrm{D}$, in the absence of distortion, and if the data was collected in the coordinate frame of the regional strike or if the data is rotated along the strike direction, the off diagonal elements of the upper two rows of the perturbation tensor $\underline{\underline{W}}$ will vanish and the GDS principal axis (see Siemon (1997) for construction of MT and GDS principal axis) reduce to one axis parallel to the strike $\left(h_{H}\right.$ or $d_{D}=0$ in addition to $h_{D}=d_{H}=0$ ). If the strike is along $\mathrm{x}$ (north-south), it is the $h_{H}$ perturbation tensor element that is zero and $d_{D}$ is maximum over the anomaly. On the other hand if the strike is along y (east-west), $d_{D}=0$ and $h_{H}$ has a maximum over the anomaly. For a subsurface conductivity structure that is 3D no element of $\underline{\underline{W}}$ vanishes (Siemon, 1997).

To understand the characteristics of the perturbation tensor elements, we look at the currents induced in the subsurface due to an anomaly. If the subsurface is uniform, the current distributions will be the same in both $\mathrm{x}$ and $\mathrm{y}$-polarizations and the vertical field is zero implying that the anomalous field is absent. On the other hand, if there is a conductive block embedded and running say east-west in a uniform halfspace, current is diverted from the surrounding of the conductive block into it. This will lead to an increase of current concentration (current density) in the conductive block and at the same time the current density outside the block will decrease significantly (see figure $2.3 \mathrm{~b}$ ). It is a well established fact that currents in the subsurface produce secondary magnetic fields (anomalous field) (Lilley, 1982) and that the direction of a magnetic field is perpendicular to the direction of its associated current flow. Therefore if the current flow is east-west, magnetic anomalies can be observed in the northern $\left(B_{x}\right)$ direction. In such a case, the $\left(h_{H}\right)$ perturbation tensor will have the highest amplitude; the amplitude of $z_{H}$ is highest outside the anomaly. Similarly, if the anomaly is oriented in the North-South direction, the induced magnetic field $\left(B_{y}\right)$, perpendicular to current direction, leads to the highest amplitude in $d_{D} ; z_{D}$ also has its maximum outside the anomaly. In general, positive values of the perturbation tensor elements are an indication of increased magnetic field relative to the regional field and negative values designate decreased magnetic field values. To illustrate this, we inserted a conductive block $(1 \mathrm{~S} / \mathrm{m})$ oriented North-South in a 0.001 $\mathrm{S} / \mathrm{m}$ halfspace and used the 3D forward modelling code of Mackie \& Booker (1999), a modified version of Mackie et al. (1994), to calculate the perturbation tensor elements. The output is shown in the figure 2.3 below. . 
a

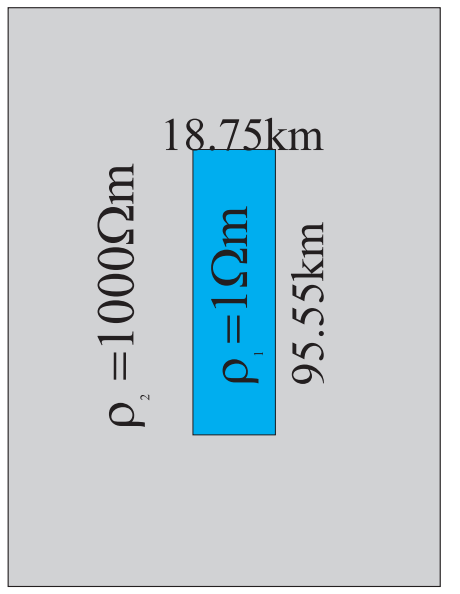

b

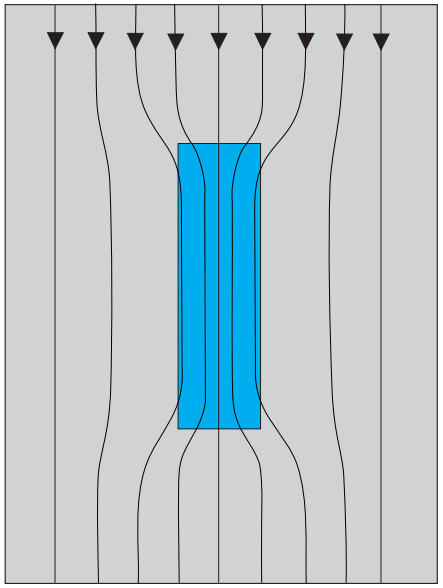

$\mathrm{C}$
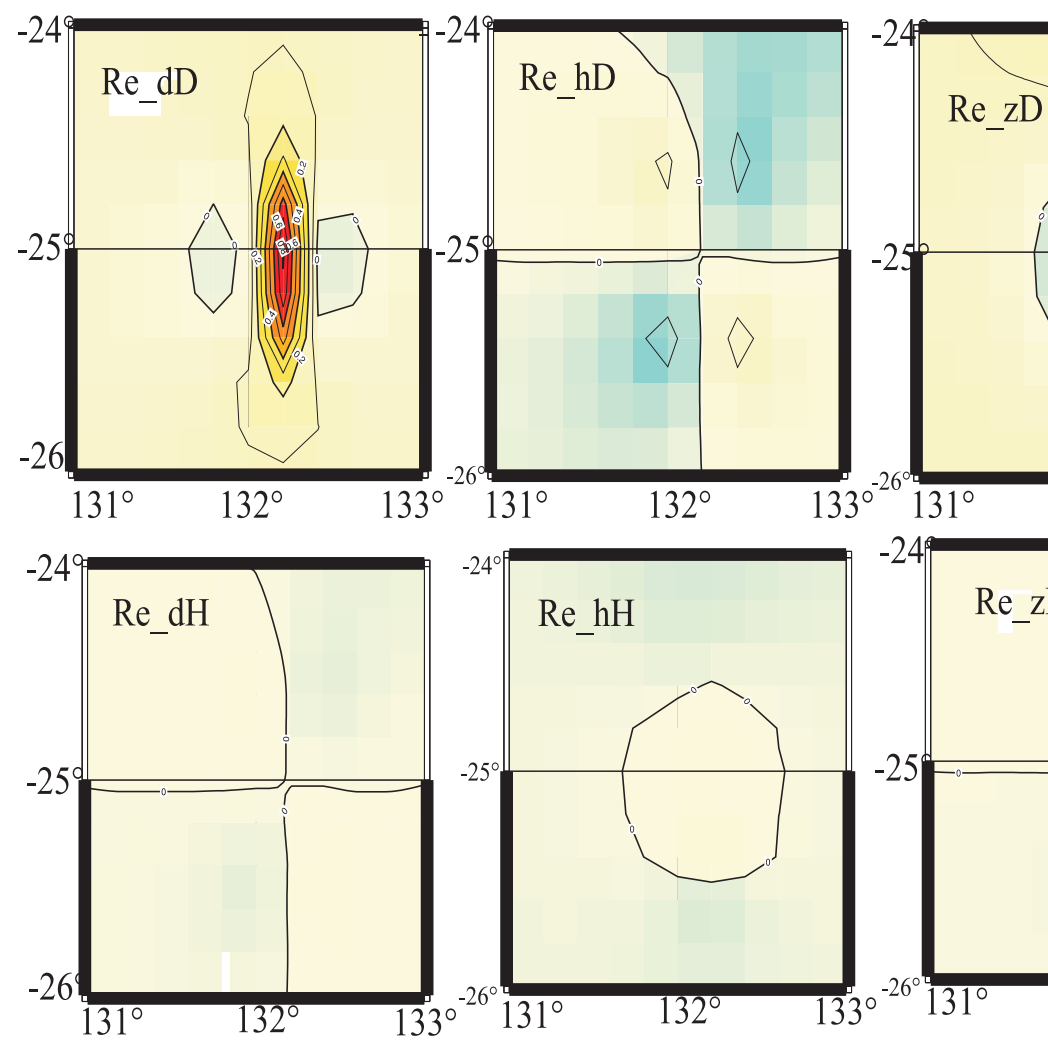

$131^{\circ}$

$133^{\circ}$

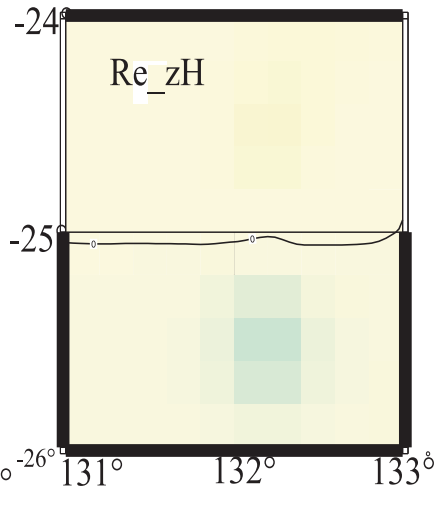

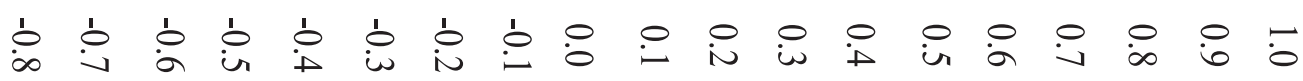

Figure 2.3: (a)Model of a conductive block with $1 \mathrm{~S} / \mathrm{m}$ in a $0.001 \mathrm{~S} / \mathrm{m}$ halfspace. (b)Sketch showing current channelling. (c) Real and imaginary parts of the perturbation tensor elements for the model in (a) calculated at a period of 32 seconds. To the left of block, $z_{D}<0$, in the middle of block $d_{D}>0$, and to the right of block, $z_{D}>0$ 
From the results obtained in figure 2.3, the following conclusion can be drawn: (i) At positions significantly removed from the conductivity anomaly all perturbation tensor elements evanesce. (ii) In the middle of the block (conductivity anomaly) only $d_{D}$ perturbation tensor element is non-zero with a maximum in the middle of the block; for a conductivity anomaly running east-west, it is the $h_{H}$ perturbation tensor element that has its maximum in the middle. (iii) The vertical $z_{D}$ perturbation tensor element changes sign with extrema at the edges of the anomaly. For an anomaly aligned East-West (not shown), it is the $z_{H}$ component that changes sign with extrema at the boundary of the anomaly. In general, the vertical magnetic field anomalies are highest outside the anomaly, however, in the vicinity of the anomaly and stay at higher values than the horizontal transfer functions. (iv) The amplitude of $z_{D}$ or $z_{H}$ at a point can increase or decrease depending the nature of a conductor placed adjacent to the point. (v) $d_{H}$ and $h_{D}$ perturbation tensors are insignificant except at the corners of the block where the currents are bended. $d_{H}$ and $h_{D}$ anomalies have as origin currents that are not perpendicular to the external (source) field.(vi) For an extended conductor, its edge can be determined from the Z-field (vertical field) and the H-field (horizontal field) can be used to measure its lateral extent.

\section{The Problem of a reference station in GDS}

For the success of GDS analysis (the calculation of GDS perturbation tensor elements, also called Schmucker's perturbation tensor elements), the reference station to which all magnetic field components are referred must be chosen with care. If the conductivity structure of the reference station is not $1 \mathrm{D}, B_{z}^{n} \neq 0$, this will affect the perturbation tensor elements at all other stations since anomalous field components will be interpreted as normal ones. That means the perturbation tensor elements will contain information about the conductivity structure both at the local site and at the reference site. In such a case the separation of information at the normal site from that at the reference site becomes difficult. Normally a permanently recording observatory or site within the array is used. All regional measurements in the surroundings of the chosen reference site should have negligible gradients in the spatial field behaviour as well in the frequency dependence.

In practice, it is not easy to know in advance a station that fulfils these conditions. Sometimes a suitable 1D station may be realised only during data analysis; if this site was not operated for the whole duration of the measurement campaign, it is not possible to use it directly as a reference site. Nonetheless, this problem can be overcome by a swap of the reference site. Leibecker et al. (2002) derived a formula to swap between the reference and field sites as follows: Equation (2.62) can be used to exchange the reference station of the array from say B to C. Using

$$
\mathbf{B}^{A}-\mathbf{B}^{B}=\underline{\underline{W}}^{A, B} \mathbf{B}^{B}
$$

and

leads to equation (2.66) below

$$
\mathbf{B}^{C}-\mathbf{B}^{B}=\underline{\underline{W}}^{C, B} \mathbf{B}^{B}
$$

$$
\mathbf{B}^{A}-\mathbf{B}^{C}=\left(\underline{\underline{W}}^{A, B}-\underline{\underline{W}}^{C, B}\right)\left(\underline{\underline{W}}^{C, B}+\underline{\underline{1}}\right)^{-1} \mathbf{B}^{C}=\underline{\underline{W}}^{A, C} \mathbf{B}^{C}
$$

where $\mathbf{B}^{A}$ is the magnetic field at station $\mathrm{A}, \mathbf{B}^{B}$ is the magnetic field at station $\mathrm{B}, \underline{\underline{W}}^{A, B}$ is the GDS perturbation tensor between stations $\mathrm{A}$ and $\mathrm{B}$ and $\underline{\underline{W}}^{A, C}$ is the GDS perturbation tensor between stations $\mathrm{A}$ and $\mathrm{C}$. 
In this project ${ }^{8}$ the observatory, Alice Spring was initially used as the reference site since we had continuous data for all the 3 campaigns (2000, 2003 and 2007). But the site ARG, see map of study area (figure 3.2) is closer to a 1D Earth than Alice Spring; we therefore used equation (2.66) which is implemented in the program gdss-swap (Leibecker et al., 2002) modified (Appendix D.3.3) to swap the reference site from Alice Spring to ARG.

\section{Magnetic distortion due current channelling and GDS strike direction}

The term current channelling is used to refer to a higher current density in one direction say x: $J_{x}=\sigma_{1} \mathbf{E}_{x}$, where $\sigma$ is the conductivity of the material and $E_{x}$ is its electric field. It is also associated to the concentration of induced currents in elongated structures with increased conductivity (figure 2.3b), and may cause distortion of geomagnetic variation fields since anomalous currents have an effect on the horizontal components of the regional electric and horizontal/vertical magnetic fields. The distortion of geomagnetic variational fields especially around the vicinity of the conductor that receives the channelled currents may lead to a modification of the geomagnetic (GDS) strike in the surroundings of the conductor. If the horizontal conductive block that receives the channelled current is oriented in a frame different from that of the regional strike, the currents channelled in it become "'anomalous"' currents due to the fact that they deviate and distort the regional electric fields (by a twist and shear distortion angles; as illustrated by Groom and Bailey, 1989). In addition, higher current flows can increase the anomalous magnetic fields. Lezaeta (2001) demonstrated that a small dike conductor embedded in a homogeneous resistive environment can produce strong intensity variations in the primary field. Current channelling has also been associated with local electrical heterogeneities (Jones 1983). Zhang et al. (1993) demonstrated that the effect of current channelling due to electric heterogeneities on the vertical magnetic field is the same as that of static shift on the electric field. Thus if the effect of current channelling on the vertical magnetic field is not taken into account, it may lead to a wrong interpretation of GDS strike direction in the vicinity of an anomaly or electric heterogeneities.

Zhang et al. (1993) derived a linear relation between the tipper vector and the impedance tensor which can be used to distinguish normal induction from current channelling by electric inhomogeneities. This linear relation was built on the foundation that measured data used to calculate the tipper vector (section 2.3.2) can be decomposed into two matrices describing induction and galvanic distortion of magnetic fields (Zhang et al., 1993; Ritter and Banks, 1998). This is possible because anomalous magnetic fields generated by the channelling is in phase with regional electric fields (section 2.3.4). The linear relation from the derivation of Zhang et al.(1993), another form of equation (2.33), is given below.

$$
\left(T_{x}, T_{y}\right)=\left[\left(T_{x}^{o}, T_{y}^{o}\right)+\left(Q_{z x}, Q_{z y}\right) \underline{\underline{Z}}^{o}\right]\left(\underline{\underline{I}}+Q_{h} \underline{\underline{Z}}^{o}\right)^{-1}
$$

where $\left(T_{x}, T_{y}\right)$ and $\left(T_{x}^{o}, T_{y}^{o}\right)$ are the observed (measured) and regional tipper vectors respectively. $\left(Q_{z x}, Q_{z y}\right)$ is the response of a distorter acting on the regional horizontal electric field to produce the current channelling effect on the vertical magnetic field (Zhang et al., 1993). The term $Q_{h} \underline{\underline{Z}}^{o}$ (which is frequency dependent, due to the frequency dependence of $\underline{\underline{Z}}$ ) represents the twist of the horizontal magnetic field created by current channelling.

\footnotetext{
${ }^{8}$ Magnetotellurics and Geomagnetic Depth Sounding in South Eastern Australia (Queensland)- Evidence for the Tasman Line?
} 
Zhang et al. (1993) further simplified the right side of equation (2.67) into two independent terms, the regional tipper distorted by near-surface structure and the local tipper valid for periods sufficiently long such that the local structure can be considered free of induction. Due to the fact that equation (2.67) contains the regional impedance tensor, it is possible to use the induction vector data from an array of magnetovariational (geomagnetic) data to extract a regional strike of the conductivity structure independent of the MT techniques (Ritter and Banks, 1998).

Using the idea that the anomalous magnetic field $\mathbf{B}_{a}$ generated by a distorted electric field is in phase with the regional electric field (section 2.3.4, equation 2.56), Ritter and Banks (1998) expressed the anomalous magnetic field as in equation (2.57). The regional electric field in equation (2.57) was then replaced by the product of the regional MT impedance tensor $\underline{\underline{Z}}_{R}$ and the horizontal magnetic field $\mathbf{B}_{R}$ base on the assumption that regional fields are uniform or that the local anomaly is adequately removed either horizontally or vertically from the regional conductivity boundary (Ritter, 1996) to get :

$$
\mathbf{B}_{a}=\underline{\underline{C}} \cdot \underline{\underline{Z}}^{R} \mathbf{B}^{R}
$$

A further assumption leading to equation (2.68) is that the regional vertical magnetic field $B_{z}^{R}$ is spatially homogeneous over the study area, and possibly small or of negligible magnitude. This means that the spatial variation of the measured vertical component $\left(B_{z}^{a}=B_{z}-B_{z}^{R}\right)$ is due to local distortion only. Ritter and Banks (1998) then showed that the phase shift between the anomalous and regional magnetic fields is equal to the phase of the regional impedance: $\varphi\left(B^{a}, B^{R}\right)=\varphi\left(Z^{R}\right)$. From equation (2.68) the anomalous vertical field is given by:

$$
B_{z}^{a}=\left(C_{z x}, C_{z y}\right) \cdot \underline{\underline{Z}}^{R}\left(\begin{array}{c}
B_{x}^{R} \\
B_{y}^{R}
\end{array}\right)
$$

where the information on the regional structure is contained in the impedance tensor $\underline{\underline{Z}}^{R}$ and $\left(C_{z x}, C_{z y}\right) \underline{\underline{Z}}^{R}$ is the local magnetic response function. Since the distortion parameters $\left(C_{z x}, C_{z y}\right)$ are real quantities, the phase information contained in the the local response function is completely related to the regional MT impedance tensor. Equation (2.69) can be used to determine the regional strike as follows:

In the measurement coordinates, equation (2.69) also contains rotational terms; assuming a $2 \mathrm{D}$ structure, we get:

$$
B_{z}^{a}=\left(C_{z x}, C_{z y}\right) \beta_{\alpha_{R}} \underline{\underline{Z}}^{R} \beta_{\alpha_{R}}^{T}\left(\begin{array}{c}
B_{x}^{R} \\
B_{y}^{R}
\end{array}\right)=\left(C_{z x}, C_{z y}\right) \beta_{\alpha_{R}}\left(\begin{array}{cc}
0 & Z_{x y} \\
Z_{y x} & 0
\end{array}\right) \beta_{\alpha_{R}}^{T}\left(\begin{array}{c}
B_{x}^{R} \\
B_{y}^{R}
\end{array}\right)
$$

$\left(C_{z x}, C_{z y}\right) \beta_{\alpha_{R}} \underline{\underline{Z}}^{R} \beta_{\alpha_{R}}^{T}$ has the same form as $\underline{\underline{\beta}}_{\alpha} \cdot \underline{\underline{D}} \cdot \underline{\underline{Z}}_{2 D}^{\prime} \cdot \underline{\underline{\beta}}_{\alpha}^{T}$ from equation (2.51) and leads to a similar expression (equation 2.53) for the strike. However, if we follow the approach of Ritter and Banks, equation (2.70) can be expressed as:

$$
B_{z}^{a}=A^{l} B_{x}^{R}+B^{l} B_{y}^{R}
$$

where $A^{l}=C_{z x}^{\prime \prime}\left[-Z_{x y} \sin \alpha_{R} \cos \lambda+Z_{y x} \cos \alpha_{R} \sin \lambda\right]$ and 
$B^{l}=C_{z x}^{\prime \prime}\left[Z_{x y} \cos \alpha_{R} \cos \lambda+Z_{y x} \sin \alpha_{R} \sin \lambda\right]$

with $C_{z x}^{\prime \prime}$ magnetic distortion parameters in the measurement coordinates. Therefore, the components $A^{l}$ and $B^{l}$ of the local transfer function depend solely on the regional impedances $Z_{x y}$ and $Z_{y x}$, the strike of the local inhomogeneity $\alpha_{l}$, the strike of the regional structure $\alpha_{R}$, their difference $\lambda=\alpha_{l}-\alpha_{R}$, and a real distortion parameter. In practice, if we measure magnetic variation over an inhomogeneity and if magnetic distortion is present as is often the case, equation (2.71) substitutes for the commonly used equation by Schmucker (1970) (provided previous assumptions still hold), equation (2.63) and in particular:

$$
B_{z}^{a}=z_{H} \cdot B_{x}^{R}+z_{D} \cdot B_{y}^{R}
$$

Consequently, the real induction arrows might indicate neither the local strike, nor the regional one, but a mixture of both.

To get the regional strike, we solve equation (2.71) for two geometric configurations (regional strike parallel or perpendicular to local strike):

$\lambda=0^{\circ}$

$$
\begin{gathered}
A^{l}=C_{z x}^{\prime \prime}\left[-Z_{x y} \sin \alpha_{R}\right] \\
B^{l}=C_{z x}^{\prime \prime}\left[Z_{x y} \cos \alpha_{R}\right]
\end{gathered}
$$

$\lambda=90^{\circ}$

$$
\begin{aligned}
& A^{l}=C_{z x}^{\prime \prime}\left[Z_{y x} \cos \alpha_{R}\right] \\
& B^{l}=C_{z x}^{\prime \prime}\left[Z_{y x} \sin \alpha_{R}\right]
\end{aligned}
$$

Solving equations (2.71) and (2.72) simultaneously leads to:

$$
\tan \alpha_{R}=-\frac{A^{l}}{B_{l}}
$$

and using equation (2.70), leads to:

$$
\tan \alpha_{R 1}=-\frac{z_{H}}{z_{D}}=\tan \alpha_{l}
$$

Similarly solving equations 2.73 and 2.74 simultaneously and using equation (2.70) gives :

$$
\tan \alpha_{R 2}=\frac{z_{D}}{z_{H}} \text { and } \quad \alpha_{R 2}=\alpha_{l}-90^{\circ}
$$

Equations (2.78) and (2.79) show that the strike direction (local or regional) is independent of magnetic distortion parameters, $C_{z x}^{\prime \prime}$ and $C_{z y}^{\prime \prime}$. A similar conclusion has been made by Ritter and Banks (1998).

On the other hand, if one returns to equation (2.68), the perturbation tensor $\underline{\underline{W}}$ can be expressed as follows

$$
\underline{\underline{W}}=\underline{\underline{C}} \cdot \underline{\underline{Z}}^{R}=\left(\begin{array}{cc}
h_{H} & h_{D} \\
d_{H} & d_{D}
\end{array}\right)
$$


The phases in each column of the perturbation tensor are the same. To obtain the strike direction, a corresponding analysis analogous to that of the impedance tensor could be used. If this is done, the following equations,

$$
\beta_{g d s}=\frac{1}{2} \operatorname{artan}\left(\frac{\left[E_{1}, T_{2}\right]-\left[T_{1}, E_{2}\right]}{\left[T_{1}, E_{1}\right]+\left[T_{2}, E_{2}\right]}\right)
$$

$\beta_{g d s}$ represents the GDS strike angle of the regional structure obtained from the perturbation tensor. This is equivalent to equation (2.53) and

$$
\eta_{g d s}=\frac{\sqrt{\left|\left[E_{1}, T_{2}\right]-\left[T_{1}, E_{2}\right]\right|}}{\left|E_{2}\right|}
$$

is the GDS phase sensitive skew corresponding to equation (2.55) (Fluche,1983; Leibecker, 2000) where $T_{1}=h_{H}+d_{D}, T_{2}=h_{D}+d_{H}, E_{1}=h_{H}-d_{D}$ and $E_{2}=h_{D}-d_{H}$. Equation (2.81) gives the strike direction (independent of the MT technique). We can use the expression for the GDS strike direction to demonstrate the effect of 'magnetic distortion' due to current channelling on the GDS strike and show that the strike direction is modified at the boundary between a conductive anomaly and a resistive host due to current channelling.

To calculate the GDS strike direction, we maximise the $d_{D}$ perturbation tensor with respect to the other horizontal perturbation tensor elements. An amplitude $A_{s}$ indicating the strength of the strike in the north-south direction is therefore given by

$$
A_{s}=\left|d_{D}\right| /\left(\left|h_{H}\right|+\left|h_{D}\right|+\left|d_{H}\right|\right) ;
$$

a large amplitude indicates strong current in the north-south direction. Below is the strike direction calculations for the model in figure 2.3a. 


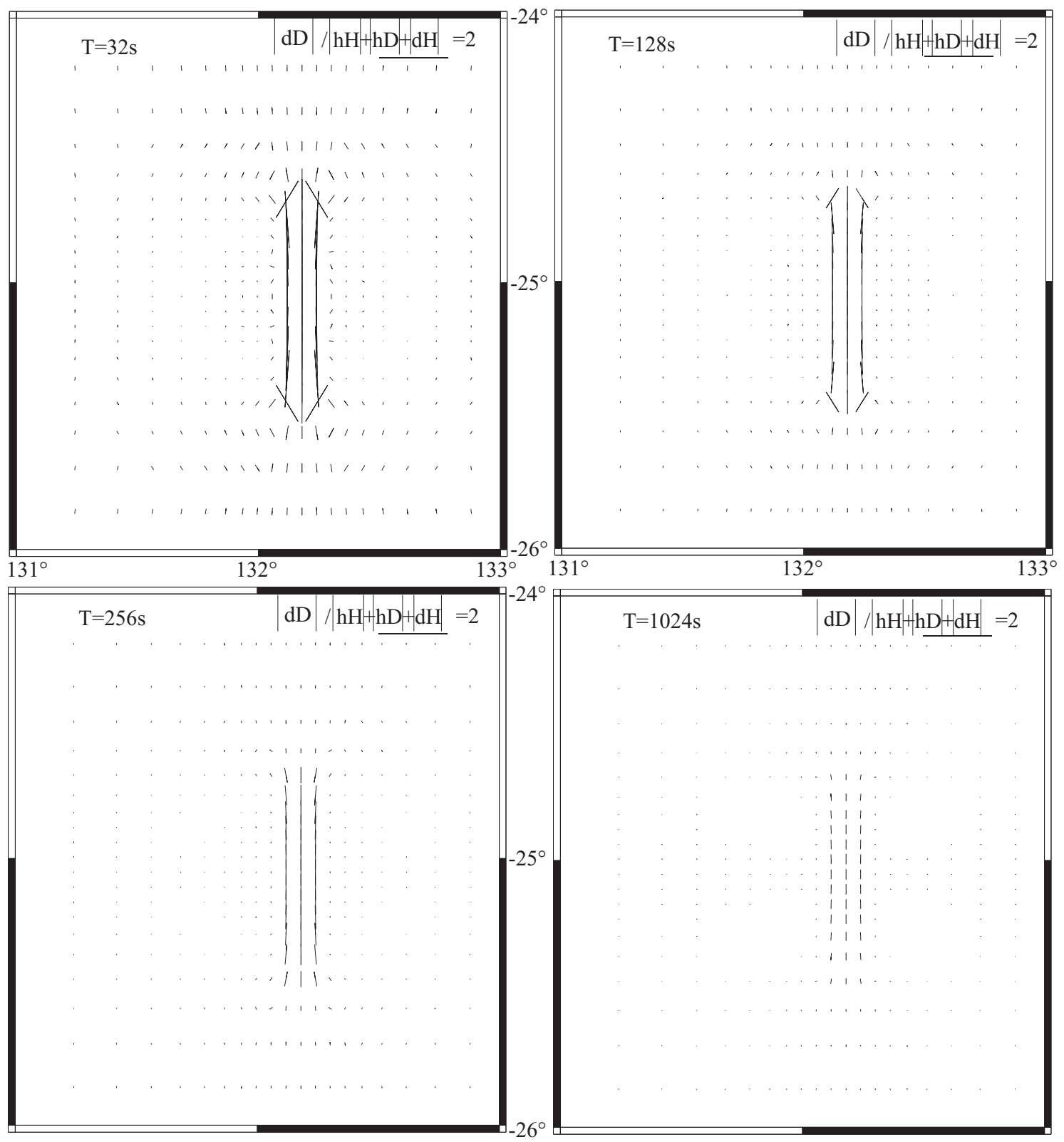

Figure 2.4: GDS strike angles for the model in figure 2.3a at periods labelled in the figure. Strike angles were calculated by maximising the $d_{D}$ element with respect to the other horizontal perturbation tensor elements. 
The results obtained in figure 2.4 show the effect of current channelling on the GDS strike angles. At a period of $32 \mathrm{~s}$ corresponding to the period at which the effect of the conductive block of the model in figure 2.3a is strongly felt, the strike direction is not entirely northsouth as expected from the model. The strike direction is distorted at the edges due to the bending of currents at the edges of the conductive block as a result of current channelling. Inside and further away from the conductive block, the strike direction is clearly north-south as expected. At these points there is no distortion of geomagnetic variation fields. However, the magnitude of $A_{s}$ is much greater within the conductive block than at points further out. This should be understood since we expect $d_{D}$ to be largest within the block. At longer periods, the contrast between the conductive block and its host is much smaller. The fields become somehow homogeneous as the effect of current channelling become minimal leading to an entirely north-south strike direction inside and at the edges of the block at a period of $1024 \mathrm{~s}$. What looks like a double edge sword at shorter periods disappears at longer periods. This can be explained in terms of a strong effect of magnetic distortion at short periods (periods where the contrast between conductive block and the host is large leading to current channelling). At longer periods, the contrast is small and the fields become homogeneous as already mentioned.

Observe that at $1024 \mathrm{~s}$, for points further away from the conductive block, $A_{s}$ is negligible. At this period, all horizontal perturbation tensors are almost the same and the idea of a strike direction is meaningless.

The observations above from synthetic data lead to current channelling distortion analysis. This analysis together with the observations from the synthetic data will help us explain the GDS strike angles calculated from our measured data; strike angles especially around the Carpentaria anomaly (figure 3.15) are not exactly north-south as expected. The current channelling analysis will also facilitate inference of the presence of elongated conductor(s) in our study area

\subsection{Current channelling distortion analysis}

Some of the concepts discussed here are taken from Lezaeta (2001). Figure 2.3b gives a clear picture of current channelling into a conductive block embedded into a more resistive host. From the figure, the higher current density is along the long axis of the conductor. If in addition currents induced elsewhere prevail, these can contribute to enhance the current density in the conductor. This will lead to a strong local current channelling effect. As illustrated in the figure 2.3b, the regional electric fields are strongly distorted (especially at the boundaries between the conductor and its resistive host) and polarised in one single direction due to a high concentration of currents that are channelled into the conductive block. An increased current flow in a particular preferable direction certainly depends on the conductivity distribution. Previous work to demonstrate current channelling can be found from articles published by Babour and Mosnier (1980); Edwards and Nabighian (1981); Jones (1983).

Based on the above defined model of a highly conductive small block of limited lateral extension as the current channeller, the EM-field boundary conditions between the conductive block and its host contribute to current channelling analysis. To understand explicitly 
how the fields change at the boundary between the conductive block and the host medium, lets modify the model in figure $2.3 \mathrm{~b}$ and assume that the strike in the conductive block is different from that in the host and take the long strike axis $(\mathrm{x})$ of the anomalous coordinate system as the local azimuth. The practical meaning associated with this kind of distortion can be understood through a thorough examination of the boundary conditions accomplished for the EM-fields intersecting two different conductivity domains, the elongated conductive block with conductivity $\sigma_{1}$ and the resistive host (regional electrical structure) with conductivity $\sigma_{2}$ (figure 2.3a). The current density in the conductive block due to channelling increases with increase in the conductivity contrast $\left(E_{\perp}^{2} / E_{\perp}^{1}=\sigma_{1} / \sigma_{2}\right)$.

Since we want to study the fields located near the centre of the anomaly in order to analyse current lines along the x-axis (north-south in our case), we study the fields located near the centre of the anomalous conductor. The anomalous conductive structure is assumed electrically thin (with respect to the field penetration depths in the anomaly) so as to neglect the effects of induction; therefore an electrostatic effect dominates (Lezaeta, 2001). Moreover, the regional EM-fields are assumed uniform in the anomalous conductor so as to satisfy the boundary conditions between the anomaly and the resistive host. The boundary conditions fulfil by electric $\left(\mathbf{E}_{1,2}\right)$ and magnetic $\left(\mathbf{B}_{1,2}\right)$ fields at an interface between two media of different electrical conductivities $\left(\sigma_{1}, \sigma_{2}\right)$ are as follows:

1) $\hat{n} \cdot\left(\sigma_{2} \mathbf{E}_{2}-\sigma_{1} \mathbf{E}_{1}\right)=0$ (The normal component of the current density is continuous).

2) $\hat{n} \times\left(\mathbf{E}_{2}-\mathbf{E}_{1}\right)=0$

3) $\hat{n} \times\left(\mathbf{B}_{2}-\mathbf{B}_{1}\right)=0$

4) $\hat{n} \cdot\left(\mathbf{B}_{2}-\mathbf{B}_{1}\right)=0$

where $\hat{n}$ defines the unit vector normal to the interface, in our case, pointing from region 1 to region 2. It may be important to not that these boundary conditions hold provided the permeability and relative dielectric constant in the two media are the same.

As mentioned earlier, if the long axis of the conductive block (figure 2.3a) is $\mathrm{x}$ and $\hat{n}$ is perpendicular to it, from boundary condition 1 (BC1) the following applies for the electric fields at the interface:

Since $\sigma_{1}>\sigma_{2}$ implies $\hat{n} \cdot \mathbf{E}_{2}>\hat{n} \cdot \mathbf{E}_{1}$. This is so since $J_{y}$, the normal component of the current density, must be continuous,

$$
J_{y 1}=J_{y 2}
$$

where $J_{y 1}=\sigma_{1} E_{y 1}$ is the current density inside the conductor and $J_{y 2}=\sigma_{2} E_{y 2}$ is the current density outside the conductor. It follows that

$$
\sigma_{1} E_{y 1}=\sigma_{2} E_{y 2}
$$

Therefore, the electric field across the conductive block (the normal component $E_{y}$ ) will jump at the boundary decreasing significantly inside the conductive block. On the other hand the magnetic component $B_{y}$ increases in the conductive region and its surrounding regions. One can observe here that electric field returns to its normal field values further away from the conductive block. Therefore the electric field across a thin conductor remains almost constant, except inside the conductive block itself where it is significantly distorted. Therefore if we examine the electric field across the thin conductor from a 
regional perspective, we may conclude that it is uniform since it will suffer only a parochial variation inside the thin conductor. A schematic overview of fields behaviour across the conductor is given in figure 2.5 (modified figure after McNeill \& Labson, 1991).

$\mathrm{a}$
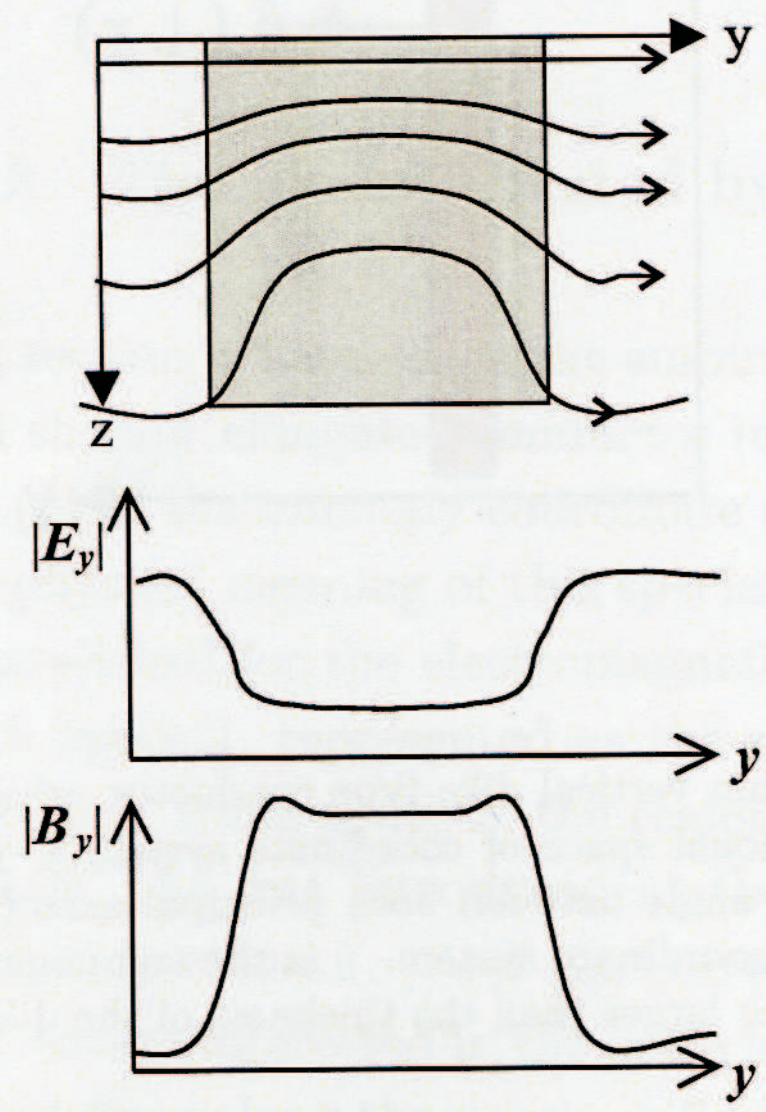

b
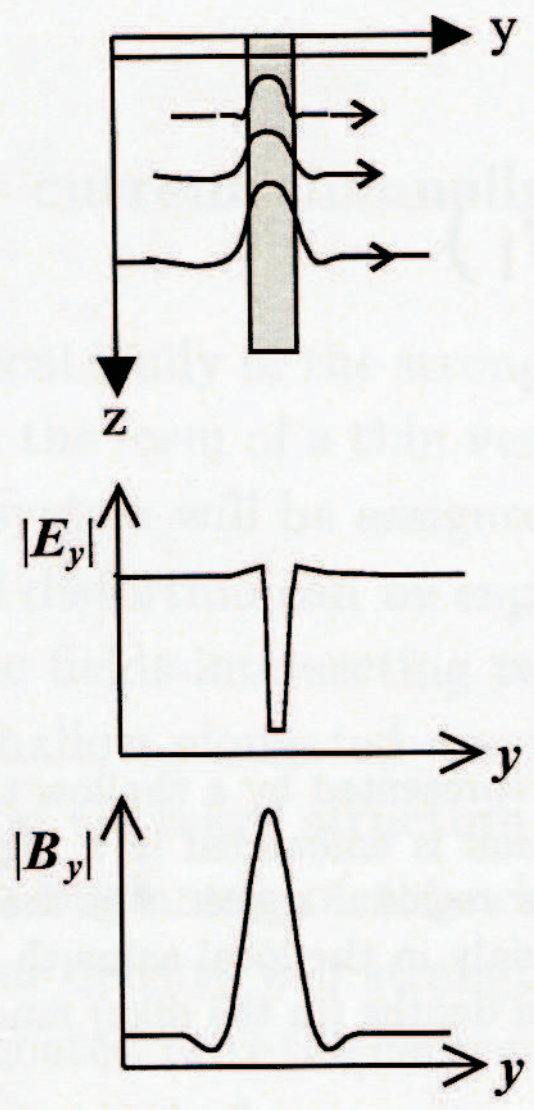

Figure 2.5: Current flow and distortion of field lines especially as it crosses the boundary between regions with laterally heterogenous conductivity. The behaviour of fields is also shown; the amplitudes of electric and magnetic fields across the electrical heterogeneity show that the electric field decreases in the conductive region whereas the magnetic component increases in the conductive region and its vicinity. Comparing a and $\mathrm{b}$, we observe that the distortion of fields increases with decrease in width of the conductive block. Modified figure after McNeill \& Labson (1991).

The modification (twist) of the fields especially at the boundary between the two domains will certainly affect the current direction, hence the strike at the boundary of the two media.

Since the electric field inside a conductor decreases significantly in magnitude (figure 2.5) 
and may even turn to zero in the case of a perfect conductor as explained above, it can be deduced from boundary condition 1 as follows: In the case where $\sigma_{1} \gg \sigma_{2}$ then $\left|\mathbf{E}_{1}\right| \ll\left|\mathbf{E}_{2}\right|$ i.e.the magnitude of the electric field $E_{1}$ inside the conductor is less than that of the electric field $E_{2}$ in the resistive host. McNeill \& Labson (1991) had also shown that the tangential electric field in the resitive host decreases significantly in magnitude compared to the normal electric field component outside the thin conductive block (in the resistive host) as a result of a high conductivity contrast between the thin conductor and its host.

BC3 tells us that the magnetic field component parallel to the boundary $\left(\hat{n} \times \mathbf{B}_{1,2}\right)$ is constant. However, if we analyse the TE-polarization mode of two dimensionality (section 2.3.1), it leads us to the result in figure 2.5 which shows that the magnetic field perpendicular to the boundary $\left(\hat{n} \cdot \mathbf{B}_{1,2}\right)$ increases in the conductive region. That means the normal electric field and the normal magnetic field are in anti phase inside the thin elongated conductor which implies that the normal electric field is a minimum when the normal B-field is a maximum (figure 2.5).

We conclude that for a small conductor embedded in a resistive space, the currents are strongly distorted at the boundaries of the conductor leading equally to a strong distortion of the fields at the boundaries independent of induction effects. This will also lead to a modification of the impedance tensor at the boundary between the conductor and the resistive host; therefore we expect a distortion of the strike angles in the vicinity of the boundary as shown in figures $2.3 \mathrm{~b}$ and 2.5 (top). A complete discussion of the channelling model is not within the scope of this thesis but we can safely conclude that a horizontally elongated conductor oriented similarly to the regional axis increases the current channelling and magnetic effects in the presence of a nearby regional high conductivity zone. 


\section{Previous work and description of study area}

The Australian Plate, one of the major tectonic plates of the Earth is made up of a number of different tectonic blocks: The major ones include Archaean (Pilbara and Yilgarn Cratons in the west and Gawler Craton-South Australia) and Proterozoic (the Capricorn Orogeny in the west; the Kimberley, the Arunta block, the McArthur Basin and the Mount Isa block in the North; the Adelaide and Coompana blocks in the South), blocks in the western and central parts and relatively younger terrains (Phanerozoic) in the east (Myers et al., 1996; Betts et al., 2002). There is a general decrease in the age of the rocks of the continent with distance eastward (Grey \& Foster, 2004). Phanerozoic east Australia consists of the Tasman Orogens which include Delamerian, Lachlan, Thomson, and New England Orogens (Betts et al., 2002). According to Grey \& Foster (2004), the Delamerian Orogen, southeast (550-470 Ma) (figure 3.1) is the oldest while the far eastern New England Orogen (310-210 Ma) is the youngest; that is the Orogens were formed at different periods from west to east.

According to a study by Betts et al. (2002), Archaean Cratons ${ }^{9}$ constitute the oldest regions of the Earth with the oldest known rocks of age greater than $2500 \mathrm{Ma}$. These Archaen rocks hold information that dates far back to the early geological time (when supercontinents existed). Every continent is said to have these Archaean cratons which act as rigid crustal blocks and provide the substrate on which more accretion (addition of landmass) occurs. The Australian plate has Pilbara and Yilgarn Cratons as its own nuclei. Figure 3.1 is a map that summaries neatly the major tectonic blocks of the Australian plate discussed above.

${ }^{9}$ long-lived stable parts of continents 


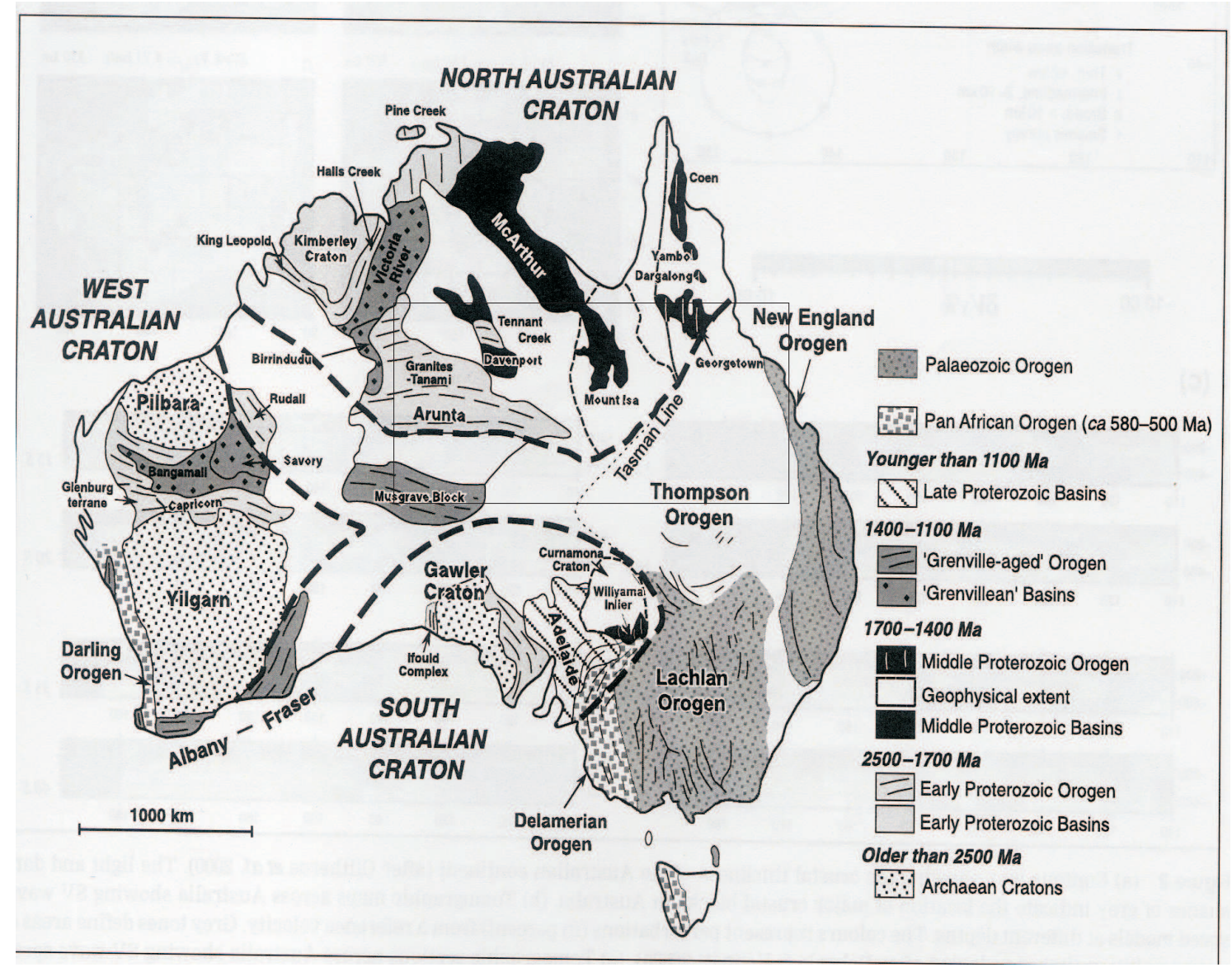

Figure 3.1: Terrane map of the Australian Plate emphasizing the major blocks(Modified figure after Betts et al.(2002). The area in rectangle indicates this region of interest.

For the Palaeoproterozoic era, an approximate time frame in the interval (2500-1600)Ma, tectonic models of the evolution and dynamics of the Australian plate have been disputatious and are subjected to further research. Veever and McElhinny (1976); Idnum and Giddings (1988) and many others interpreted Proterozoic Australia as a single intact continent. But, a more popular alternative hypothesis has been proposed by Myers et al. (1996) and supported by Betts et al. (2002) and others. Their research concludes that in the Palaeoproterozoic era, three major cratons, the north, west and south Australian cratons evolved independently by amalgamation of numerous smaller crustal fragments through tectonic processes (Betts et al., 2002). Between $1300 \mathrm{Ma}$ and $1000 \mathrm{Ma}$ these cratons amalgamated during collisional events that led to the formation of the Autralian continent, a component of the early supercontinent Rodinia (Myers et al., 1996). Then, the Centralian Superbasin (extensive intracratonic basin) developed over the junction of the north, south and west Australian cratons between about 830 and $750 \mathrm{Ma}$ (Water et al, 1995). It comprises shallow marine and fluviatile sandstones overlain successively by carbonate and evaporite deposits (Myers et al., 1996). Rifting to the east formed an epicontinental rift basin called 'Adelaide Geosyncline'. This was followed by the break-up of 
Rodinia as a result of movements along older lines of weakness and related sedimentary deposition, with the rifting apart of Laurentia and Gondwanaland along the eastern margin of Proterozoic Australia in about 750 Ma (Cengage, 2003,2006), and the subsequent formation of the Palaeo-Pacifici Ocean (Myers et al., 1996).

In a critical review to highlight the main stages of the evolution of the Australian plate, Crawford et al. (2003) also attributed the formation of older western and central Australia to amalgamation processes in the Paleoproterozoic and Proterozoic eras. Crawford et al. (2003), proceeded to aver that eastern Australia is dominated by much younger Tasman fold belts formed in the Phanerozoic era. Among Phanerozoic mountain belts, the Tasman orogenic system of eastern Australia has been described as unique. It has no throughgoing miogeocline or foreland fold and thrust belt (Coney et al., 1990). The evolution of the different Orogens that constitute present day Phanerozoic eastern Australia is a whole topic on its own. For some good literature on the evolution of the Orogens of eastern Australia, we refer the reader to papers by Coney et al. (1990); Foster and Gray (2000); Betts et al. (2002). The bottom line is that, the exposed geology of the Australian continent is composed of a collection of crustal blocks that can be broadly grouped into the Precambrian western and central cratons and the Phanerozoic eastern province. Veevers and Powell (1984a) described a series of rifting events along the eastern Precambrian Australia craton which were followed by a divergent episode. This series of rifts is the commonly called Tasman Line marking the eastern boundary of Precambrian terranes.

Hill (1951) is reputed for having first coined the term Tasman Line as the boundary between Proterozoic western and central Australia and the younger eastern Australia. Therefore Veevers (1984) is one of the early tectonic modellers who agreed with Hill's concept of the Tasman Line. Nonetheless, his own boundary was slightly different. This boundary is associated with the break up of the early supercontinent Rodinia around $780 \mathrm{Ma}$, Wingate et al. (2002). Many authors have since postulated different possible locations for the Tasman Line on the basis of outcropping basement rocks, magnetic and gravity surveys. Up to date, there is not yet any unanimously agreed location for this historic boundary. While the actual position of the Tasman Line is still evasive, structural differences between the Precambrian west/centre and the younger eastern Australia have been inferred from surface wave dispersion (Muirhead \& Drummond, 1991; Denham, 1991) and tele-seismic travel-time residuals (Drummond et al., 1991) whose origin is due to structures that must have extended to a depth of at least $100 \mathrm{~km}$. In addition, the relatively fast wave speeds associated with the lithosphere extend to around $220 \mathrm{~km}$ beneath the older Archaean and Proterozoic regions of western and Central Australia, whereas beneath the Phanerozoic regions of eastern Australia, seismic wave speeds suggest that the lithosphere is less than $140 \mathrm{~km}$ thick (Kennett, 2003). From receiver functions, Clitherore, Gudmundsson and Kennett (2000) concluded that there is evidence for intracrustal structural differences between the Phanerozoic Tasman Orogen in the east and the Precambrian cratons to the west. They went further and termed the mid-crustal discontinuity that they found in the Tasman Orogen as the upper boundary of a Proterozoic continental substrate on which the Tasman orogen accreted.

However, the relatively abrupt eastern margin mapped out by Kennett (2003) did not correlate well with the Tasman Line marking the eastern extent of current Precambrian outcrop. Since it is clear that there exist structural differences between west/centre and 
east Australia, there must exist a common boundary for the terranes. Selway, Heinson \& Hand (2006) and many others have demonstrated that electromagnetic methods can be used to delineate fundamental crustal boundaries. Therefore our objective is to use subsurface conductivity that must be different for the regions to discriminate the boundary. If the Tasman Line exists, it will have a distinct conductivity that could be resolved by electromagnetic methods. Electromagnetic methods are based on the conductivities of rocks; as a general trend old rocks (Proterozoic) are resistive whereas younger rocks (Phanerozoic era) are relatively more conductive. 


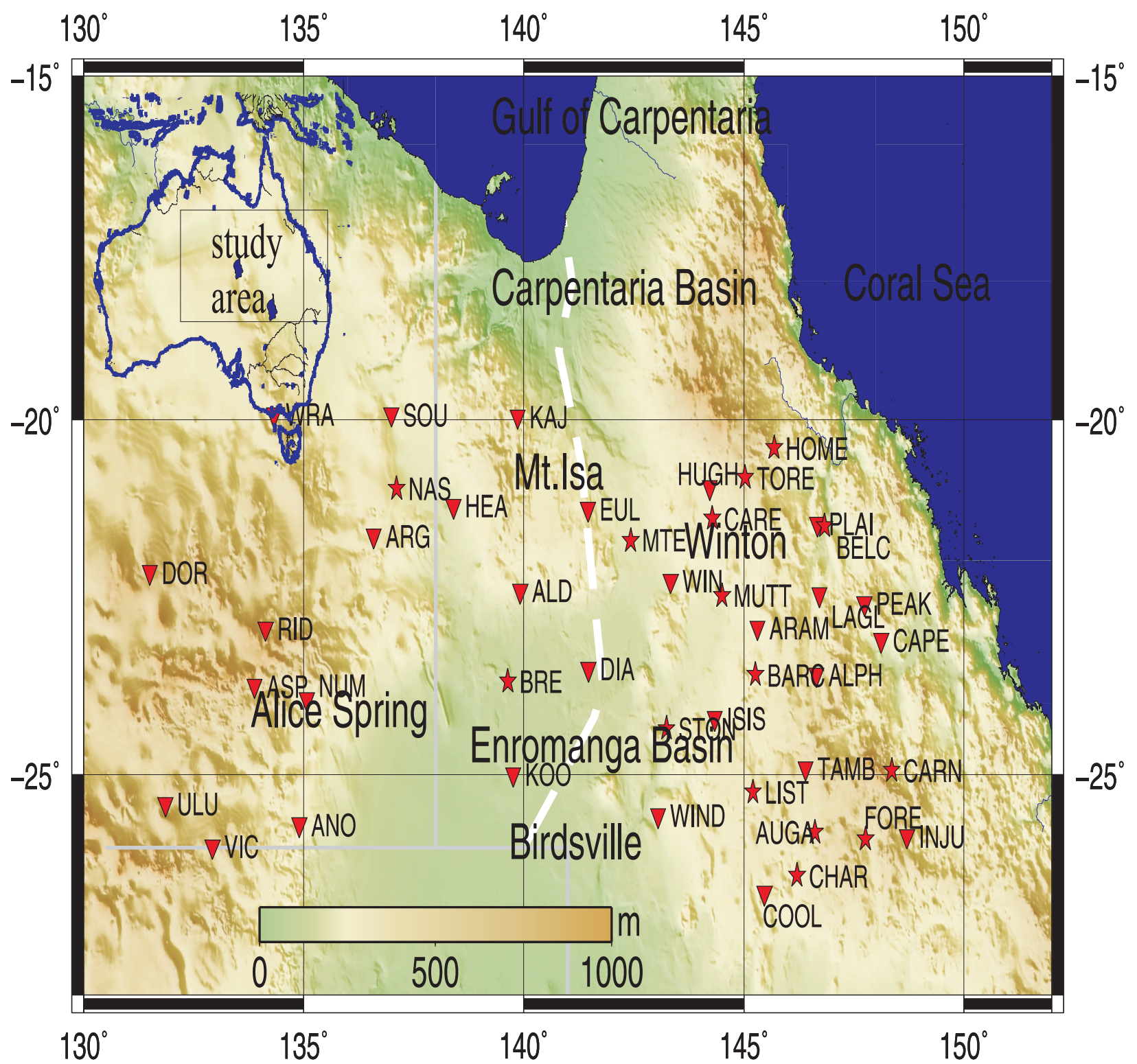

Figure 3.2: Topographic map and major geological structures of the study area. The triangles and stars represent MT sites; triangles stand for sites from which long period data were obtained while stars represent sites from which short period data were measured. The dashed line running north-south represents the Carpentaria conductivity anomaly. On the upper left corner of the figure is a small map of the Australian continent with the study area clearly shown. 
The study area (figure 3.2) is between longitude $130^{\circ} \mathrm{E}$ and $150^{\circ} \mathrm{E}$ and between latitude $19.5^{\circ} \mathrm{S}$ and 26.5S. This covers a good portion of west/central and east Australia. The most significant geological features (terranes) in this area are the Paleoproterozoic Arunta Block (figure 3.1) in the West and the Proterozoic Mt Isa Inlier in the north-east (Betts et al., 2002); the sedimentary basins notably the late Proterozoic Georgina Basin (north-west) and the Mesozoic Eromanga Basin (Chamalaun et al., 1999).

The Eromanga Basin in the interior Lowlands of north-eastern Australia is a subdivision of the Great Artesian Basin that covers parts of Queensland, the northern territory, South Australia and New South Wales. It covers an area of approximately one million square kilometres and is, therefore among the larger basins of the world (Finlayson \& Leven, 1997). The basin is covered by Jurassic-Cretaceous conductive sediments which obscures the older infra-basins which contain clues to the early evolutionary past of the whole region. The early tectonic evolution of the central Eromanga Basin region is still not well established. Like the whole Great Artesian Basin (figure 3.3) to which the Eromanga basin is part, the basin was created during the Mesozoic period, 248 to $65 \mathrm{Ma}$ (Australian Bush Hospitality Pty, Ltd) where the area slumped under the weight of an inland and it's own deposits. To balance this slumping or sagging, land around the edge, largely made of sandstone was uplifted. The sandstone gradually eroded away and was carried into the basin, resulting in a deposit of sandy sediments almost 3000 meters deep (Clitherore, Gudmundsson and Kennett, 2000). The sandstone sediments are porous and given the water content of the basin, the interconnected pore fluid makes them highly conductive. However, it is known that in the time interval between 600-130 Ma- during which the sediments were formed-Australia was part of eastern Gondwanaland along with India and Antarctica (Embleton, 1984). 


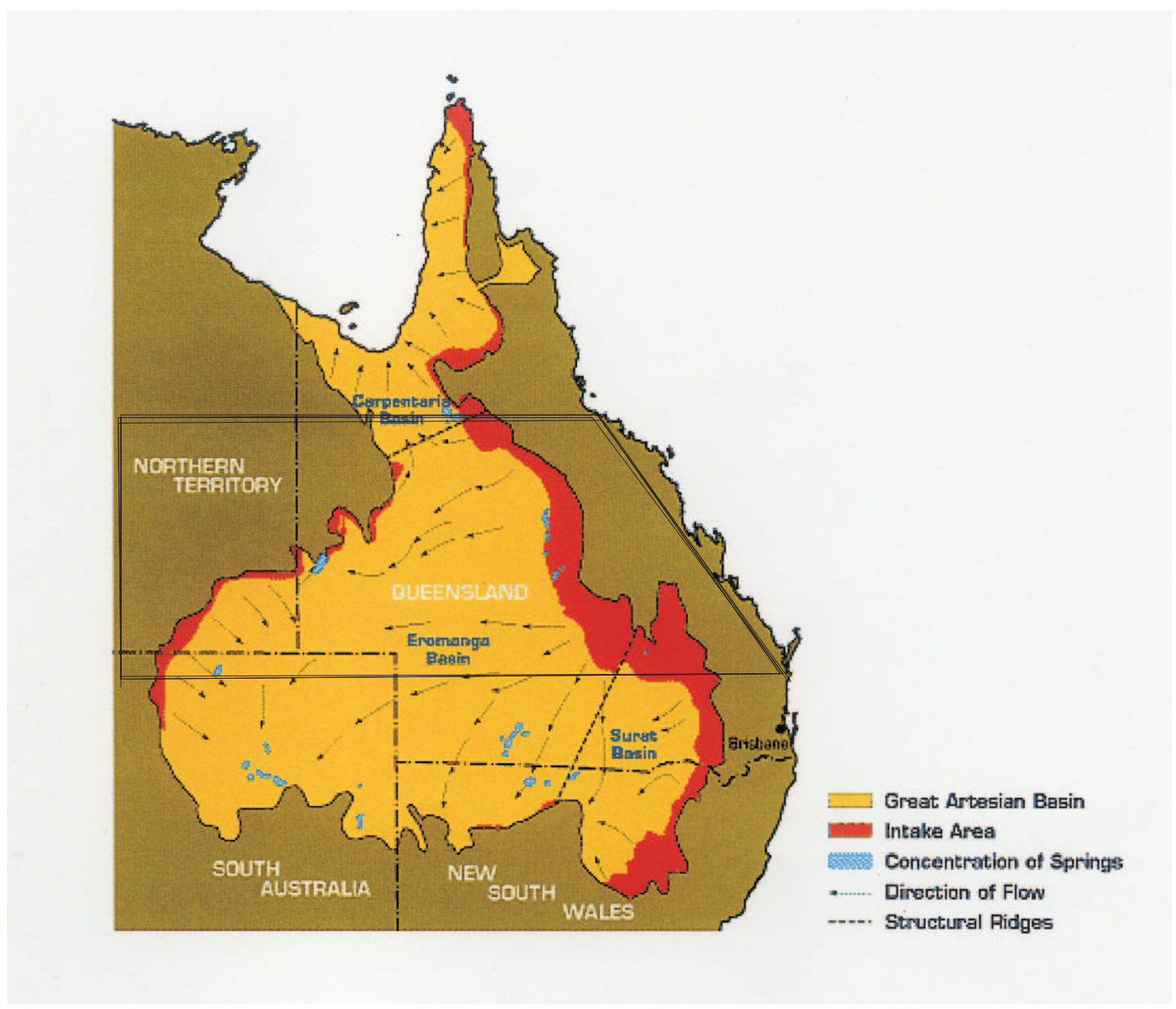

Figure 3.3: The Great Artesian Basin (adapted from Australian Bush Hospitality Pty, Ltd) showing its constituent basins (the Eromanga, the Carpentaria and the Surat basins. The trapezium represents our study area.

The north-eastern Eromanga Basin contains a stratigraphic record of extensive marine flooding across the Australian continent during the early Cretaceous (Campbell \& Haig, 1999) commonly called the chalk period (145-66 Ma). It is estimated that 60 percent of the present land mass of Australia was engulfed by the sea during Aptian-Albian (Frakes et al., 1987 \& repeated in Campbell \& Haig, 1999). This signifies the largest marine flooding over the continent since the Cambrian-Ordovician era (Veevers, 1990; Campbell \& Haig, 1999 ). As a consequence, most sediment assemblage in the area occured during the cretaceous flooding.

The accumulation of the sediments now stands as a stumbling block for geophysical methods in mapping underlying basement crustal-scale architecture or geology. The thickness of the sediments of the Great Artesian Basin which includes the Eromanga Basin, the Carpentaria and Surat Basins ranges from between at least 100 on the Basin extrimities to over 3,000 metres in the deeper parts of the Basin (GABCC, 1998). Details of the tectonic evolution of the area can be found in Harrington (1974), Kirkegaard (1974), Murray 
and Kirhegaard (1978), Day et al. (1978, 1983), Veevers (1984), Korsch et al. (1988), Campbell \& Haig (1999) and Clitheroe, Gudmundsson and Kennett (2000)

The Mt. Isa terrain of northwest Queensland which is Proterozoic in age is a major Precambrian element of the Australian continent. Betts et al. (2006) referred the Mt. Isa Inlier as an excellent natural laboratory to study Proterozoic tectonic processes. The Inlier is surrounded by the Mesozoic sedimentary environments which include the Eromanga, Georgina and Carpentaria Basins. It distinguishes itself as one of the world's best records of Proterozoic Orogenesis (O'Dea et al., 1997). It displays outstanding examples, perharps the world's most spectacular examples of intracontinental rift development, wrench faulting and extensive metasomatism (O'Dea et al., 1997).

After the Barramundi Orogeny, an event that marks the deformation, metamorphism and cratonization of the earliest Proterozoic between about 2000-1900 Ma in north Australia (Page \& Williams, 1998), the Mount Isa Inlier, between about $1800 \mathrm{Ma}$ and $1600 \mathrm{Ma}$ experienced a protracted period of intracontinental rifting characterized by extensive orogenic felsic magmatism and uplift (Carter et al., 1961; Smith, 1969; Page et al., 1994; O'Dea et al., 1997 and references therein; Page \& Williams 1998; Betts et al., 2006 ). The Mount Isa region has a high density of faults, a large proportion of which shows evidence for right-lateral strike-slip displacement (O'Dea et al., 1997). The Mt. Isa Belt is truncated by a rifted margin formed during the break up of the supercontinent Rodinia in about 750Ma (Cengage, 2003, 2006).

According to geological literature on the geodynamic evolution of the Australian plate, there are similarities between the Mount Isa Inlier in northeastern Australia and Proterozoic blocks in southern Australia. These similarities suggest a physical link between northern and southern Australia throughout the Proterozoic era. Common features have also been identified between the Isan Orogen in the north and the Olarian Orogen (south). O'Dea et al. (1997) attributed the features common to both Isan Orogeny and Olarian Orogeny to a high probability that the Mount Isa terrain and Willyama Complex represent the locus of a compressional event. Notwithstanding, from the magnetic and gravity anomaly maps of the region (figures 3.4 and 3.5), the Mount Isa block has distinct geophysical features which may be largely due to the juxtaposition of rocks with contrasting geophysical properties. O'Dea et al. (1997) has observed that dense and high magnetic susceptiblity mafic volcanic rocks are often in contact with less dense and lower magnetic susceptibility granitoids and basement rocks. He attributed this to advanced rifting and associated basaltic volcanism and/ or more underplating of mafic material within the Mt.Isa Inlier than in other Proterozoic blocks in the south.

Another important feature of Mt. Isa is its thin sediments which are rich in world class metals such as Lead-Zinc-Silver (Pb-Zn-Ag) orebody deposits (Neudert \& Russel, 1981). Detail discussions of the geology of the area may be found in Demead et al. (1974) and Graeme, Kenneth and Kinny (1997). More recent geological studies include that of Southgate (2000) and Betts et al. (2006).

Similar to the Mount Isa Inlier, the rocks of the Arunta block are Proterozoic in age. Not much is known about the evolution the Arunta block and documentation about this block is relatively poor. Only conflicting models exist for the evolution of the Arunta Block. As an example, Betts et al. (2002) pointed to accretion processes at the southern boundary 
on the north Australian craton whereas Giles et al. (2004) attributed it to 1300 to 1100 Ma activity during rearrangements of the north and south Australian cratons.

\section{Previous studies}

In general, the Australian plate is one of the most studied. The 1990s have seen an explosion of new geophysical, geochemical and geological data for the Australian continent and the wider plate. This culminated in 2000 into a multidisciplinary volume ${ }^{10}$ that was published on the evolution and dynamics of the Australian plate, with papers from different sub disciplines of earth sciences. Previous knowledge of the Australian plate can be summarised from results obtained from seismic tomography, gravity surveys, magnetic anomaly data and electromagnetic induction studies which are discussed below.

\subsection{Seismic tomography results}

A significant number of seismic experiments covering the whole of Australia have been conducted in the past and of recent, (Finlayson \& Leven, 1987; Finlayson et al., 1990; Simons et al., 1999; Debayle \& Kennett, 2000; Kennett, 2003; 2004, Fishwick et al., 2005; Vaughan et al., 2005; Cayley et al., 2011 and others) and these constitute good summaries for one to work on.

From seismic data, Kennett (2003), in agreement with other authors, concluded that the cratonic region in the centre and west Australia is underlain by a thick mantle lithosphere extending to around $210 \mathrm{~km}$ depth with fast wave speed. Beneath the eastern Phanerozoic outcrop, the lithosphere is thinner (less than 140km). In spite of that, some authors hold that the thickness of the lithosphere varies to a great extent in Central Australia (Simons \& van der Hilst, 2002; Collins et al., 2003). Contrary to expectations, the thickest lithosphere is found in Proterozoic Central Australia rather than the Archaean Cratons in the extreme west. Simons \& van der Hilst (2002) concluded that the thickness lithosphere is not simply age dependent and suggested that, for Australia it might have been caused by tectonic erosion under the Archean parts of the lithosphere due to their locations at the edges of the continent. This assertion is contradicted by results from Collins et al. (2003); similar to Proterozoic north and central Australia, they measured in addition, the thickest crust in Phanerozoic southeastern Australia. Clitherroe et al. (2000b) had also found a distinct transition from thick crust below central Australia to thinner crust of about 35-40 km in eastern Australia. However, the average continental crustal thickness is $38 \mathrm{~km}$ (Collins et al., 2003). Similar to lithospheric thickness, the thickest crust is found in Proterozoic Central Australia. However, the location of the Moho is hardly exact. This is due to the fact that most of the data used to quantify the Moho (Crustal depth) do not resolve well any local changes in Moho depth that may occur at tectonic boundaries (Collins et al., 2003). For this reason tectonic boundaries are defined on the basis of geophysical character and tectonic age and do not necessarily coincide with major changes in crustal thickness.

Seismic results have also been used in an attempt to delineate the Tasman Line; at greater depths (upper mantle), the transition zone between lower wave speeds to the east and faster

${ }^{10}$ sponsored by Santos and Woodside 
velocities in Central Australia was proposed as the Tasman Line (Kennett et al., 2004). They could not draw any conclusion about the Tasman Line because the transition zone in wave speed changed both vertically and laterally and did not coincide exactly with that of the conventionally recognised Tasman Line. Nonetheless, the Tasman Line extends across New Guinea and the associated change in lithospheric character (thick and strong to the west, thin and weak to the East) was also an important influence on Mesozoic-Cenozoic evolution of the region.

\subsection{Gravity surveys}

Gravity at the Earth's surface is approximately constant (The precise strength of the Earth's gravity varies depending on location). In addition to location, it may vary slightly depending on the density of rocks that lie close to the surface. Gravity is slightly greater where dense rock formations lie close to the surface. Therefore, measurements using a gravity meter (also called gravimeter) respond mostly to changes in the density of rocks within the first few kilometres of the Earth surface. Crustal structures can be revealed through the analysis of gravity data since regional gravity data highlights major crustal structures. When regional gravity (due to large-scale geologic features as opposed to local or residual gravity anomaly due to smaller-scale geologic structures) data are collected and plotted, continuous contour lines may be used to infer the lateral extent of underlying rocks. Individual positive gravity anomalies are mainly associated with relatively dense metamorphic belts, especially if they are connected with mafic volcanics and mafic or ultramafic intrusives. Negative anomalies on the other hand are caused by granite batholiths, felsic volcanics, and low density sedimentary sequences.

In eastern Australia, linear gravity highs in the central parts of several basins are almost certainly related to rifting during their early development. These gravity ridges are attributed to emplacement of elongated mafic intrusions within the crust along the rift axes and piles of mafic volcanics at the base of basin sequences. In some basins the linear gravity highs appear to have a structural origin related to uplift or over thrusting of basement (Murray et al., 1989).

The general geologic and tectonic framework of Australia is known in general terms from numerous gravity studies and compilations. Gravity measurements began in Australia during 1950-51, when the Australian Bureau of Mineral Resources, Geology and Geophysics (BMR) established the Australian National Gravity Network. The reconnaissance gravity survey of Australia initiated by BMR with a maximum station spacing of $11 \mathrm{~km}$ began in 1959 and was completed in 1974, spanning a period of 15 years (Fraser et al., 1976). According to the results from the reconnaissance gravity survey, the Australian mainland is divided into nine main gravity divisions; see Fraser et al. (1976) for details. From the the nine divisions, division 5 (the Proterozoic Mt. Isa Geosyncline) is of interest to this work and I will like to quote from Fraser et al. (1976). "The Proterozoic Mount Isa Geosyncline in western Queensland correlates with a broad northerly trending regional gravity high of complex contour pattern. Proterozoic rocks are exposed only over a small part of the gravity high, but the gravity feature indicates that the block extends to the south as basement under the Great Artesian Basin. The feature terminates abruptly about 
$250 \mathrm{~km}$ south of the exposed part of the geosyncline, against a region of lower Bouguer anomaly level"

Between 1976 and late 1990s, detailed coverage of the continent continued, this time with a more reduced station spacing $(5 \mathrm{~km})$. In 1992 the first edition of a new series of the gravity anomaly map of the Australian region was published as an enhanced pixel image (Morse et al., 1992). The second edition (the Australian anomaly map that covers the entire continent) was published in 1997 based on data from approximately 700.000 land gravity stations (Murray et al., 1997). Murray \& Petkovic (2001) used data from more than 900.000 gravity observations collected from almost 1000 surveys and updated the version of Murray et al. (1997) to give a detailed gravity anomaly map of the Australian region. For details, see Murray et al. (2001) and Milligan et al. (2003).

Queensland, our area of interest has been extensively covered by gravity measurements carried out by the Geological Survey of Queensland (GSQ). Since 2005, 41589 gravity stations have been collected under the Smart Exploration initiative and 29788 under the Smart Mining Future Prosperity initiative. In 2007, Geoscience Australia completed a 3550 station regional gravity survey over the Cooper Basin in the North. Queensland's modern era of gravity survey commenced with the Bowen-Surat data collection in 2005 which was part of the Smart Exploration Initiative (SMI). Gravity work by SIM in 2007 on Mt. Isa area (most highly prospective areas for gold and base metal exploration in Queensland) by private contractors was both $2 \mathrm{~km}$ and $4 \mathrm{~km}$ station interval data. Complete coverage of western Queensland at $4 \mathrm{~km}$ station interval or better was finalised with the completion of the Westmoreland-Normanton gravity survey in August 2008 (Geological Survey of Queensland). 


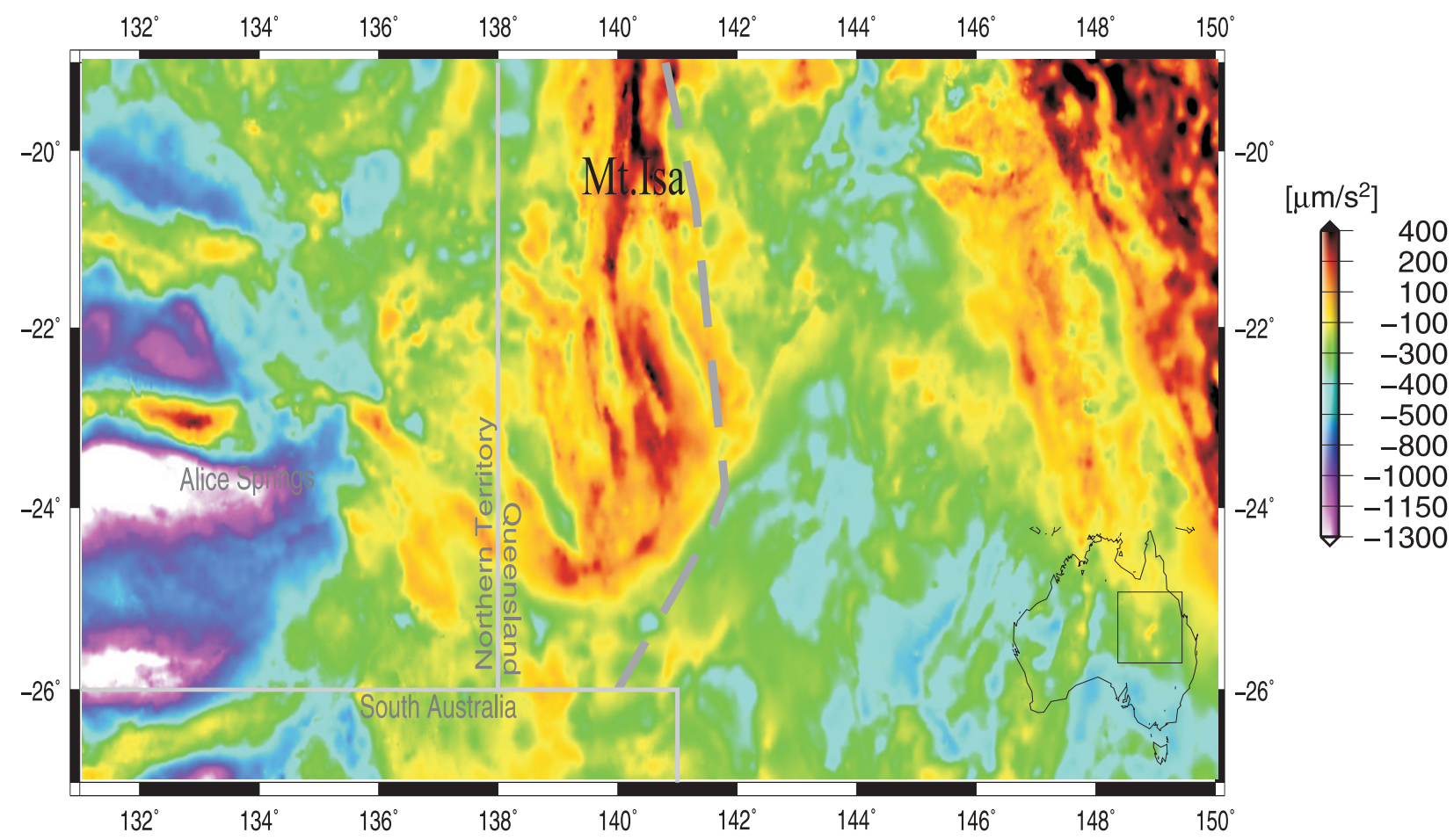

Figure 3.4: Gravity anomaly map of the study area. The data range from blue (low) to red (high). The dashed line marks the Carpentaria conductivity anomaly as mapped out by Chamalaun et al. (1999). Gravity data from Geoscience Australia (Tracey et al., 2008)

Figure 4.3 shows the Bouger anomaly map of the study area. The most conspicuous feature is an elongated structure of gravity highs accompanied by strong gravity gradients along $140^{\circ} \mathrm{E}$, known as the Barramundi Worm (Bierlein \& Betts, 2004). This structure extends several hundred kilometres further south away from the Mt. Isa block and therefore is not in agreement with surface geology. Fraser et al. (1976) and Gunn et al. (1997) have argued that the gravity feature indicates that the rocks of the Mt. Isa Block could have continued under cover for several kilometres south. The density of rocks along the elongated structure of gravity highs is definitely distinct from surrounding regions; hence the structure stands out as a boundary between regions of contrasting densities (Bierlein \& Betts, 2004)

Gravity highs can result from crustal thinning beneath rifts (Fraser et al., 1976 \& Gunn et al., 1997). Therefore the broad gravity highs below the Mt. Isa Block could be a remnant of rifting processes active during the Baramundi compressive orogenic event (Betts et al., 2002).

\subsection{Magnetic anomaly data}

Magnetic anomalies result from subsurface variations in magnetic susceptibility. The shape of magnetic anomalies, unlike that of gravity is controlled by more than one factor (sub- 
surface distribution of susceptibility and orientation of the Earth's main field) and hence is more difficult to construct compare to the gravity ones. A common method of magnetic survey is aeromagnetic survey, a geophysical survey carried out using a magnetometer aboard or towed behind an aircraft. A magnetometer is attached to a flying aircraft and it registers the sum total of magnetic variation intensities due to the temporal effects of the constantly varying solar wind, spatial variations in the Earth's magnetic field (regional magnetic field) and the local effect of magnetic minerals in the Earth's crust. When the solar wind and regional effects are subtracted from the total, the resulting aeromagnetic map gives the spatial distribution and relative abundance of magnetic minerals in the upper levels of the crust.

Due to the fact that different rock types differ in their content of magnetic minerals, the resulting magnetic map makes the visualization of the geological structure of the upper crust in the subsurface possible. This also applies especially for the spatial geometry of groups of rock and the presence of faults that separate tectono-stratigraphic terranes. Aeromagnetic data is very important in comparing lithological trends and structural elements if basement rocks below shallow cover and sedimentary basins are imaged. Mafic dikes-dike swarms can be readily discerned from aeromagnetic images and therefore may be traced from one craton to another.

The Bureau of Mineral resources (BMR), Geology and geophysics, now Geoscience Australia started Airborne magnetic surveys as early as 1951. Nonetheless, it took quite sometime, more than 25 years before the first edition (an incomplete but a predecessor of the new series of maps now published as 'Magnetic Anomaly map of Australia') of the magnetic map of Australia, in contour map form, was published in 1976 (BMR 1976a). In 2004, the 1:25 million scale version, together with composite grids of the Australian region at $250 \mathrm{~m}$ and $400 \mathrm{~m}$ grid cell spacing of the fourth edition of the Magnetic Anomaly Map of Australia was released by Geoscience Australia (Miligan \& Ravat, 2004). In July 2010 Geoscience Australia released the 5th edition, a full-colour complete new compilation of Total Magnetic Anomaly Map of Australia at a scale of 1:5 million (Milligan, 2010). It has eight million line-kilometres more than were acquired for the previous edition released in 2004. This map is a significant achievement in the representation of Total Magnetic Intensity (TMI) data across the Australian continent compared with preceding editions. It gives the possibility to investigate the magnetic anomaly configuration of the entire continent at once. This makes the thorough examination of the tectonic framework of Australia possible, even in large areas without outcrop. As would be expected, these magnetic anomaly maps have shown that the strength of the magnetic field varies across Australia. For details of Magnetic field patterns and interpretation across Australia, Miligan \& Ravat (2004) stands as a good reference. Figure 3.5 shows the total magnetic anomaly map of the studied region. The 'hotter colours' (e.g. red) show areas of stronger magnetic field and the 'cooler colours' (e.g. dark blue) show areas of weaker magnetic field. The map points out strong variations from negative to positive magnetic anomalies in the Mt. Isa Block and south of it. Its delineation is similar to that in the gravity anomaly image (figure 3.4) with strong magnetisation along $140^{\circ} \mathrm{E}$ giving additional support to the rifting hypothesis of the evolution of the Mt. Isa block. 


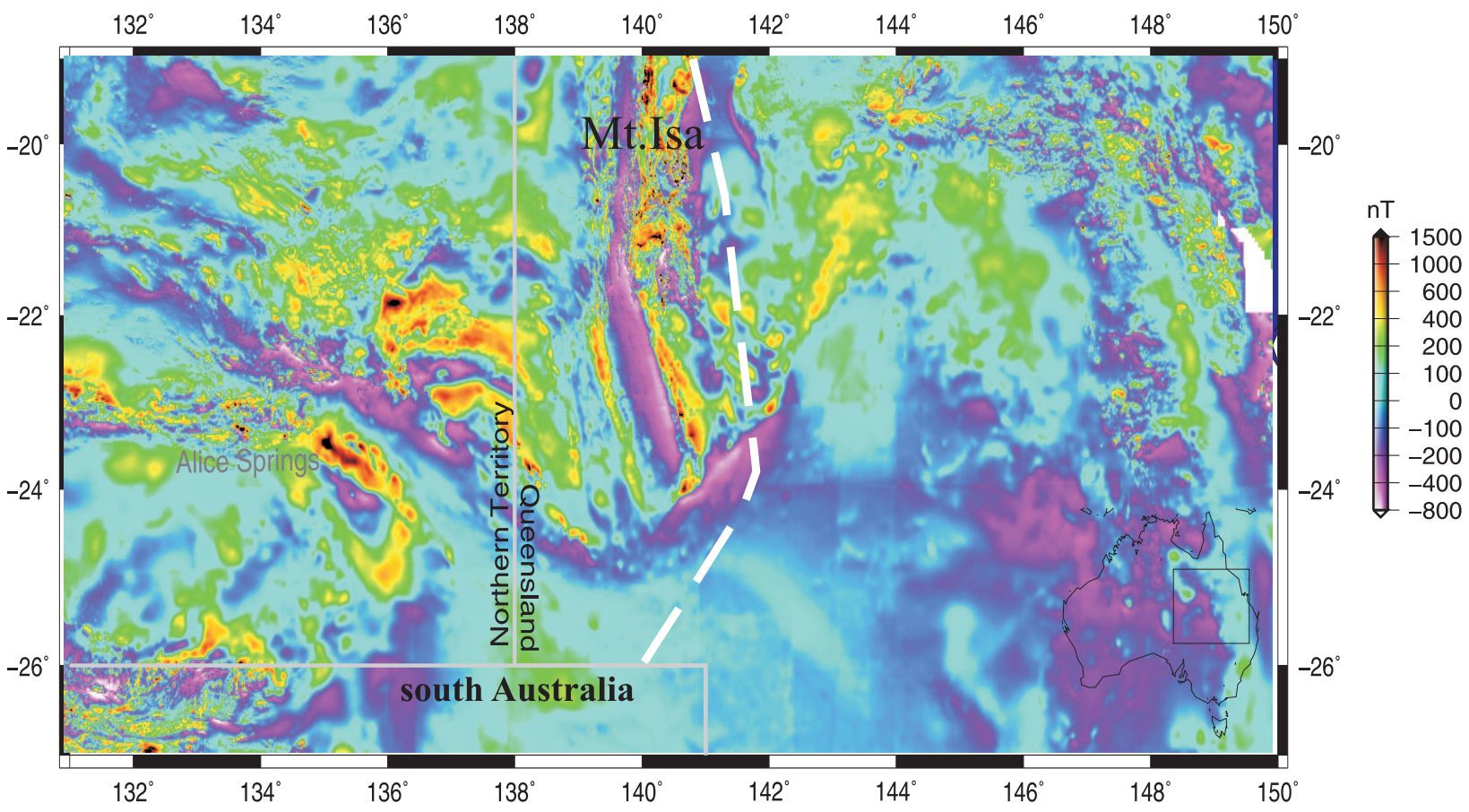

Figure 3.5: Total magnetic anomaly map of the study area using aeromagnetic data (regional effects included). The data range from blue (low) to magenta (high). The dashed line marks the Carpentaria conductivity anomaly as mapped out by Chamalaun et al. (1999). Magnetic anomaly data from Geoscience Australia (Milligan, 2010)

\subsection{Geomagnetic field Fluctuations over Australia and Coast effects}

An overview of geomagntic field fluctuations over Australia is very important if one seeks a better interpretation of electromagnetic induction over the continent. First, this overview will give a general picture of geomagnetic activity and field patterns over the continent which will ease interpretation of magnetic induction with regards to Inland and coast effects ${ }^{11}$. Secondly since electromagnetic behaviour in the continent is complicated by the effect of its surrounding sea waters, care can be taken when interpreting the vertical fields at distances not too far from the seas. In general, understanding the origin of the earth's magnetic variations with time and magnetic geographical homogeneity or nonhomogeneity is very important in EM induction prospecting. This is because variations of the Earth's magnetic field with time can lead to an error during magnetic surveying (Lilley, 1982). One way to handle the difficulties such fluctuations may pose is to understand the origin of the fluctuations and their geographical homogeneity or nonhomogeneity. This is one of the main reasons why the geographical uniformity of magnetic fluctuations were measured in the early 80s over several different areas of the Australian continent as part of research projects to examine natural electromagnetic induction taking place in the Earth

\footnotetext{
${ }^{11}$ an effect caused by induced electric currents flowing in the oceans and large seas and characterised by anomalous vertical fields
} 
(Lilley, 1982). Equally, knowledge of the crustal magnetic fields ${ }^{12}$ is very important as a geophysical exploration tool to determine local geology.

The geomagnetic fluctuation observations are often classified and put into two classes: those due to a 1D Earth (horizontally layered) and those as a consequence that local geology deviates from one-dimensionality and therefore is two- or even three-dimensional.

A large body of literature has been written on magnetic fluctuation patterns over Australia in general; comprehensive summaries have been given by Lilly (1976); Whellams (1996); Lilly et. (1999); Hitchman et al. (1998, 2000) where they described the magnetic fluctuation patterns over Australia as spatially non-uniform.

There are three factors (Frequency dependence, Horizotal polarization and source field uniformity) that influence the characteristics of any particular fluctuation patterns (Lilley, 1982) for both Australia and other continents. The three factors are fundamental properties of the primary source fields which are present in the ionosphere and magnetosphere, external to the conducting Earth. Horizontal polarization and Source field uniformity that may be specific for Australia is discussed below.

Horizontal polarization: In classic papers by Parkinson (1959) and Wiese (1962), a relationship exists between the vertical magnetic field fluctuation component and the horizontal magnetic field variation component (equation 2.33). The strength of the vertical magnetic field fluctuation component depends on the geographic direction in which the horizontal magnetic fluctuation component is varying (direction of horizontal polarization of the magnetic fluctuation). For Australia, the direction of the horizontal magnetic fluctuation usually depends on its surrounding waters. Australian magnetic fluctuation patterns can then be summarized into 3 categories (Lilley, 1982) (a) The coast effect- A coast forms a distinct boundary between a highly enhanced conducting sea and a less conducting land. Electrical conductivity of both seawater and the subsurface of the oceans are quite different from that of the continents (Seawater is highly conductive and material under oceans has a high water content and since rocks with a greater water content are more conductive, material under oceans is more conductive). Parkinson \& Jones (1979) termed this effect as the geomagnetic coast line effect. Near the coast, the horizontal magnetic fluctuation strikes at right angles across the coastline and produce a vertical magnetic fluctuation whose strength is proportional to it, that means near the coastline, the vertical magnetic field is significantly high and may overshadow the effect from near inland conductors especially at long periods. The effect may be due partly to electromagnetic induction in the seawater with elevated conductivity as a result of extensive eddy currents and in addition probably due in part to a contrast between continental and sea-floor geology (Lilley, 1982). If regional structures are uniformed, the strength of the vertical magnetic fluctuations decreases with distance from the coast (White \& Polatajko 1978). b) Inland magnetic fluctuations are smooth where the Earth's electrical conductivity is 1D. In Australia, there is a smooth magnetic fluctuation across northern Victoria and southern New South Wales (Lilley, 1982 ). Inland, in places where magnetic fluctuation patterns are anomalous and non uniform, there must be the presence of electrical conductivity anomalies in the continental geology. In Australia, many of such areas with

\footnotetext{
${ }^{12}$ fields arising from magnetic materials in the Earth crust commonly called anomalous field
} 
anomalous conductivity anomaly exist and were first mapped out by the 1976 magnetometer array experiment of Woods and Lilley (1979). In these regions, the total-field magnetic fluctuation patterns can change significantly over distances. c) Offshore magnetic fluctuations are different from those inland and at the coast. Milligan \& Barton (1997) attributed this to the effect of the edge of the continental shelf. Offshore itself, there are magnetic variations associated with ocean swell (Lilley et al., 2004)

Source-field uniformity- The source field (section 2.1) is not spatially uniform over the entire Earth; the source field observed in high-latitudes may be different to that observe in mid-latitudes. Therefore, for electromagnetic induction surveys over large distances, one will need to test the source field spatial uniformity. In order to judge a field observed at a station in a certain area as being anomalous or to determine to what extent it is anomalous, a general distribution of the source field must be understood to some extent (Honkura, 1978). On the other hand, even over the same region (same latitude), because of the lack of consistent structure in the Earth's ionosphere and magnetosphere, where the source field arises, non uniformity in the source field turns to be inconsistent among magnetic events.

Source field non-uniformity are strong in the auroral and equatorial regions of the earth and might arise as a problem for electromagnetic induction studies. Australia lies in the mid-latitudes and non-homogeneities in magnetic storm source-fields over the continent are generally very weak, indeed inconsequential, with regards to the needs of induction prospecting. However, in his study, Mutsushita (1967) stated that for the case of the quiet daily variation, the source-field pass directly over Australia approximately at the same rate as the sun. Therefore, there is the presence of non-uniformity for the daily variations source-field over Australia. Notwithstanding, Lilley (1982) argued that the non-uniformities introduced by the source field of the magnetic quiet daily variation are insignificant for for the purpose of EM induction studies. In addition, within a certain area of local extent, the source field can be treated as being spatially uniform (Honkura, 1978). These arguements indicate that for the Australian continent, one can safely consider the source field as homogeneous over distances that are common for a single MT or GDS survey. Further more, Singh (1980) stated that the effect of the source filed will be approximately the same at stations separated by distances small compared to the source field. In our case, the distance from the source field is far larger compared to the separation between our stations. If the source field over Australia is unform, then variations of the anomalous magnetic field over the continent will be entirely due to crustal magnetic field as well as due to induction in its surrounding oceans.

\subsection{Previous electromagnetic array studies}

Magnetometer array investigation commenced in Australia in 1970. Gough et al. (1974) pioneered electromagnetic induction studies in Australia with the electromagnetic induction surveys in southern Australia which covered a greater part of the Gawler Craton and Adelaide Geosynline (figure 3.1). The complex pattern unravelled by the study was interpreted as two crossing conductors (Gough et al., 1972). One of the conductors was later described to have followed the trend of the Flinders Ranges Anomaly (Chamalaun, 
1985, 1986). The second one was at the time poorly defined and was named after the Australia-Wide Array of Geomagnetic Stations (AWAGS) experiment (Chamalaun \& Barton, 1993) as the Arckaringa Anomaly (see figure 1.1). Gough's pioneer work was closely followed by the electromagnetic array studies in the near coasts of south-east Australia (Lilly \& Bennett, 1972; Woods and Lilly, 1979). Other early electromagnetic induction surveys include geomagnetic induction array mappings in central Australia which covered the South-western part of Queensland and geomagnetic depth sounding experiment in the central Flinders Ranges of South Australia (Chamalaun, 1985).

The 1976 magnetometer array experiment of Woods and Lilley (1979) discovered a major anomaly in south-western Queensland which the 1977 array of the same investigators, Woods \& Lilley (1980) followed up in detail and speculated that the southwest Queensland anomaly might be connected to the original Flinders Ranges Anomaly and that it might extend further north (figure 1.1). The above pioneering electromagnetic induction studies paved the way to AWAGS experiment (Chamalaun \& Barton, 1993) which covered the entire Australia continent with site spacing of about $275 \mathrm{~km}$ when magnetic Observatory sites are included. From AWAGS results, a northerly extension of the Arckaringa/Southwest Queensland Anomaly was supported (Chamalaun \& Barton, 1993) and identified as the Gulf of Carpentaria Anomaly (figure 1.1b). The general conclusions from the AWAGS (Chamalaun \& Barton, 1993) were as follows: The broad conductivity structure of Australia appears to be dominated by the conducting sediments of the major sedimentary basins and that within the basins local structures produce current concentrations that give rise to a narrow band of possible interconnected conductors, the intracratonic conductive zone (figure 1.1). Three separate conductivity anomalies define the Australian intracratonic zone, the Southwest Queensland Anomaly (mapped by 1976 and 1977 array studies, Woods and Lilley, 1979; 1980); the Canning Basin Anomaly (mapped by the 1985 magnetometer array of Chamalaun and Cuneen (1990)) and the Flinders Ranges Anomaly.

Woods \& Lilley (1980), followed by the AWAGS experiment had pointed to indications of a north-south striking of the Carpentaria conductivity anomaly. In particular, the results of the AWAGS observations postulated that the south western Queensland anomaly (see figure 1.1b) possibly continues north from Birdsville (figure 3.2), but that there was also a probability that it could still possibly head northeast, taking a significantly different route across major features of the Geology of Australia . In order to map out the details of this anomaly and its relationship to fundamental Australian geology, in 1995, Chamalaun et al. (1999) deployed additional magnetometers with smaller station spacing in the region. Their results supported the interpretation of the AWAGS data by chamalaun and Barton (1993b) for this part of Australia (the anomaly continues north from Birdsville). Nonetheless, the exact position (horizontal position) of the anomaly and its relationship to central and east Australian tectonic boundary (Tasman Line) has always been ambiguous and inconclusive. Curious to unearth the details and motivated by the hypothesis that major conductivity anomalies may mark old plate margins (Gough, 1983), Lilley et al. (2003) installed more magnetometer arrays in the area in 1997 and concluded the position of the anomaly at a boundary between the mega-elements of the Australian plate, the North Australian and North Queensland mega-elements. Nonetheless, their investigations still did not resolve the controversial Tasman Line (introduced in section 3.6), a key objective of this project. 
Part of the data analysed in this project has already been analysed by Simpson (2002) and Hanekop (2006). Simpson (2002) used wide-spaced long-period MT measurements in the west of the present study area and investigated a possible connection between electrical anisotropy in the upper mantle and plate-tectonics and concluded that electrical anisotropy directions correlate well with the plate motion. However, the mechanisms leading to the anisotropy are still ambiguous (Simpson \& Tommasi, 2005). Because of the wide station spacing and with just six stations, the Simpson (2001) dataset did not allow for investigation of regional crustal structures. In 2003 Simpson et al. returned to the field for a new campaign that extended the earlier one to the East. The 2003 data was analysed together with that of 2000 by Hanekop (2006). Following the recommendations of Hanekop, it was necessary to extend the campaign far east before any conclusion could be made about the Tasman Line. Based on this recommendation a new campaign was carried in 2007 in eastern Australia.

\subsection{Tasman line and recent interpretation}

As already mentioned in the introduction, different authors have used different data sets and positioned the Tasman Line differently. A selection of these demarcations of the Tasman Line is given in the figure 3.6 below (borrowed from Kennett et al., 2004). 


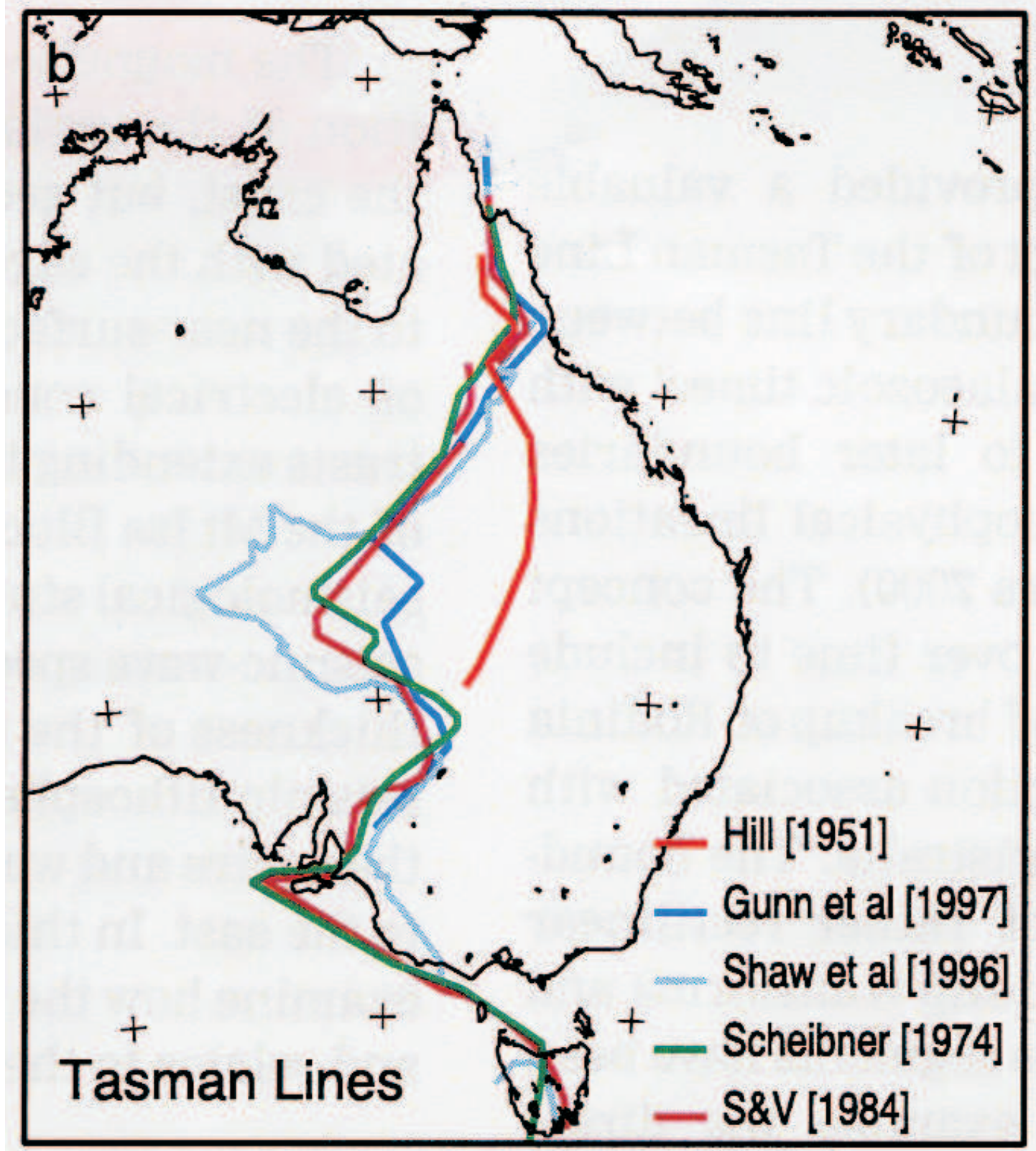

Figure 3.6: Superimposition of a number of proposed Tasman lines for comparison with our own line in later figures (modified figure after Kennett et al., 2004). 
According to Hill (1951), who first used the term, the Tasman Line was defined as the boundary line between the craton and geosyncline (Miogeosyncline) in the early palaeozoic era and the present line of junction of Pre-Cambrian rocks with early Palaeozoic rocks. According to his definition, the Tasman Line was the remnant dispositional edge of earliest palaeozoic basin systems based on outcrop ${ }^{13}$. Hill's delineation of the Tasman Line was limited in Queensland only, passing east of the Great Artesian Basin and into New South Wales at longitude $144.5^{\circ} \mathrm{E}$

As one can see in the figure, because the concept was redefined by Hill's successors, the position of the line is completely different from that first proposed by Hill. In Scheibner's 1974 paper, the concept was redefined to represent the boundary separating the more moderately deformed Phanerozoic rocks of the Tasman Fold Belt System that lie to the east from older Archean and Proterozoic cratonic blocks to the west. In his approach, a greater percentage of segments of the Tasman Line were identified as mapped faults. In places where faults could not be readily identified, he improvised geophysical lineaments (linear features in a landscape which are expressions of an underlying geological structure such as a fault or fold-aligned hills) such as the Diamantina River Lineament. Scheibner (1974) interpreted the majority of the mapped features as transformed faults imposed on the old cratonic mass during pre-Palaeozoic rifting of small blocks from the main craton.

Shaw et al. (1996) compiled gravity lineaments from Wellman (1976) to obtain their own vision of the Tasman Line as shown in figure 3.6. In a similar manner, Gunn et al. (1997) delineated the Tasman Line based on a system of rectilinear gravity and magnetic lineaments they believed represented a Proterozoic continental margin. Veevers (1984) had a completely different approach to the definition of the Tasman Line. His own Line, represented in figure 3.6 as $\mathrm{S} \& \mathrm{~V}[1984]$ was based on the concept of orogenic suture and he stated that the Tasman Line marks the suture (ocean closure) between the Precambrian craton to the west and the Phanerozoic fold belt to the east. The idea of the principal suture in eastern Australia along which rifting, break-up and subsequent active plate margin contraction occurring has been repeated in Scheibner (1998) and Scheibner and Veevers (2000). In a related approach, Li and Powell (2001) interpreted the Tasman Line as a Proterozoic rift margin and the line of break-up between the Australian (Gondwana) and American (Laurentia) sectors of Rodinia. A detailed literature review and recent interpretation of the Tasman Line can be found in Direen \& Crawford (2003). It is Veevers' definition that is of interest to us; our electromagnetic induction method can discriminate between old and young rocks even at greater depths.

As already stated in the introduction, the magnetic and gravity anomalies used in the definition of the various Tasman Lines lie in the upper part of the crust, but geophysical evidence for a contrast associated with Precambrian rocks to the west and Phanerozoic ones to the East is not confined to the near-surface and in addition, if the Tasman Line is obscured under later sedimentary cover along much of its length, any probes to delineate it must reach greater depths before any conclusion can be made.

From the definitions by previous authors as outlined above, no definition is based on electromagnetic induction. The only detailed electromagnetic induction study in the area

\footnotetext{
${ }^{13}$ a visible exposure of bedrock or ancient superficial deposits on the surface of the Earth and holds important information for understanding the geologic time scale of Earth history
} 
was by Chamalaun (1999) which was limited to just latitude $23.56^{\circ} \mathrm{S}$ and he did not investigate the Tasman Line. In his reference to the Tasman Line, this is what he had to say and I quote: "the 'Tasman Line' is a frequent marker for large-scale geological divisions of Australia (see for example Powell et al., 1994; Van der Hilst et al., 1998). However, what forms the Tasman Line takes at depth, and even its exact position in places where it is obscured by sediments, are matters yet to be determined".

This project therefore intends to use electromagnetic induction studies to shed more light on the concept of the Tasman Line especially at greater depths. To make any conclusion about the Tasman Line base on electromagnetic methods, one needs a profile of electromagnetic induction sites in the vicinity of previous delineated Tasman lines running from the Gulf of Carpentaria to at least latitude $28^{\circ} \mathrm{S}$. In chapter 4 , it was demonstrated that the data follow a trend leading to the demarcation of the Tasman Line and this is concretized in chapter 5 through modelling of the data. 


\section{Field measurements in Central and East Australia}

The data analysed in this project was measured in three different campaigns. Since the target was mantle structures, fluxgate magnetometers (consisting of three mutually perpendicular ring-core sensors enclosed in a waterproof capsule) which are suitable for long period measurements were used. In particular, the equipment were Göttingen type RAP data loggers (Steveling \& Leven, 1992) with Magson fluxgate magnetometers and Silver/Silver chloride $(\mathrm{Ag} / \mathrm{AgCl})$ electrodes. Five channels, two horizontal electric (NS and EW) and three magnetic field components (NS, EW and vertical down, Z) were recorded in the geomagnetic coordinate system. That means the measured coordinate system was settled with respect to magnetic north, thus both horizontal components are NS (X) and EW (Y) oriented. Before we proceed, a brief description of the principle of the fluxgate magnetometer might be helpful.

\section{Fluxgate magnetometer}

Its working principle is based on magnetic saturation (maximum induced magnetic field produced in the ferromagnetic bars from which the instrument is constructed: Saturation occurs when increases in the strength of the inducing field no longer produce larger induced fields). A fluxgate magnetometer consists of two bars of ferromagnetic materials that are placed parallel and close to each other. The susceptibility of the two ferromagnetic bars (cores) is significantly large in order that even relatively weak magnetic fields such as those of the Earth can produce saturation.

Each ferromagnetic core is wound with a primary coil, nonetheless, in reverse direction such that when an alternating current (that causes a large inducing magnetic field) is passed through the primary coils, the induced magnetic fields in the two cores have equal strengths in opposite directions and therefore cancel out. Nevertheless, there is a secondary coil wound over the two ferromagnetic cores and the primary coil such that the magnetic fields induced in the cores by the primary coil produce a voltage potential in the secondary coil. In the absence of the Earth's magnetic field, the voltage in the secondary coil is zero because the magnetic fields generated in the two ferromagnetic cores are equal but act against each other, i.e their effect on the secondary coil cancels out exactly.

If the ferromagnetic cores are adjusted so that they lie parallel to a component of even a weak external magnetic such as that of the Earth, one core produces a magnetic field in the same direction as the external field and restrengthen it. The other which is in opposition to the external field produces an induced field that is smaller. This difference induces a voltage in the secondary coil that is proportional to the magnetic field strength in the direction of the cores and which can be recorded.

Therefore, the fluxgate magnetometer is very sensitive and is able to measure the strength of any component of the magnetic field of the Earth by a simple re-orientation of the 
instrument so that the cores lie parallel to the desired component $B_{x}, B_{y}, B_{z}$ to be measured.

Measurement of the data began in 2000 with just six sites to the west of central Australia. Since only six sites were measured in 2000, a new campaign that used the same instruments was planned and carried out between July and October 2003 to extend the earlier one to the east. For the long period sites, a sparse site spacing of $200 \mathrm{~km}$ was appropriate with short period sites in between for the purpose of delineating crustal structures (Hanekop, 2006). GPS receivers were used for correct timing between the sites. The shortest available period obtained for the data is $8 \mathrm{~s}$. For long period sites, periods of at least $10.000 \mathrm{~s}$ could be reached with minimal error bars.

After analysing the 2000 and 2003 data, it became necessary to extend the measurements to the east of Australia. Therefore, another campaign followed in 2007. Like the previous ones, it was between July and October using the same instruments. It is also important to note at this point that data from Alice Spring Geomagnetic observatory has been used for our GDS analysis. In this case, Alice Spring was initially used as a reference site before a swap was made to a near $1 \mathrm{D}$ site (ARG in figure 3.2). Figure 3.2 shows the location of sites measured in 2000, 2003 and 2007

\subsection{Data processing}

The digital time series data collected during the MT surveys were processed using the robust remote processing code of Egbert \& Booker (1986) which is a robust single site method developed to calculate the electromagnetic transfer functions. Prior to a Fourier transform, the time series of each electric and magnetic field component was cleaned up of isolated outliers with bad data segments containing peaks manually excluded from further processing. Data were pre-whitened within a decimation scheme. Explicit expressions for MT and GDS transfer functions are given in Appendix C. For details of steps from digital time series to MT transfer functions, see Simpson \& Bahr (2005).

During the 2007 campaign, data acquired at one of the stations (COOL, figure 3.2) exhibited missing horizontal magnetic field components for a significant interval of time series. This was because the horizontal magnetic field components at this station failed irreparably. With no spare instruments it was decided to continue registering the other components of the time series in the hope that usable MT transfer functions could be recovered during processing.

We demonstrate in this section that this was actually possible and that high-quality MT transfer functions could be obtained at this site using a technique that involves (i) substituting the magnetic field components from a neighbouring site and (ii) correcting for horizontal magnetic gradients using Schmucker's perturbation tensor, $\underline{\underline{W}}$. The success of this technique obviates the need to make repeat measurements. The method utilises Schmucker's perturbation tensor, $\underline{\underline{W}}$ and is described below: 


\subsubsection{Method}

Let us consider two sites $\mathrm{A}$ and $\mathrm{B}$, where $\mathrm{A}$ is the reference site and $\mathrm{B}$ is an observation site. In MT, the orthogonal components of the horizontal electric and magnetic fields at the reference site, B are related via a complex impedance tensor, $\underline{\underline{Z}}$ (section 2.3):

$$
\mathbf{E}_{B}=\underline{\underline{Z}} \mathbf{H}_{B}
$$

The GDS transfer function between A and B is given by the relation:

$$
\mathbf{H}_{B}-\mathbf{H}_{A}=\underline{\underline{W}} \mathbf{H}_{A}
$$

From equation 4.2, the magnetic field at the observation site B is obtained to be

$$
\mathbf{H}_{B}=\underline{\underline{W}} \mathbf{H}_{A}+\mathbf{H}_{A}=(\underline{\underline{W}}+\mathbf{1}) \mathbf{H}_{A}
$$

The magnetic field at site B can then be substituted in (4.1) to obtain:

$$
\mathbf{E}_{B}=\underline{\underline{Z}}(\underline{\underline{W}}+\mathbf{1}) \mathbf{H}_{A}=\underline{\underline{Z}}_{a} \mathbf{H}_{A}
$$

From equation (4.2), the expression for the magnetic field at site $\mathrm{A}$ is given by:

$$
\mathbf{H}_{A}=(\underline{\underline{W}}+\mathbf{1})^{-1} \mathbf{H}_{B}
$$

If we substitute the expression for $\mathbf{H}_{A}$ from (4.5) in (4.4), we get

$$
\mathbf{E}_{B}=\underline{\underline{Z}}_{a}(\underline{\underline{W}}+\mathbf{1})^{-1} \mathbf{H}_{B}
$$

The new impedance tensor from equation 4.6 is:

$$
\underline{\underline{Z^{\prime}}}=\underline{\underline{Z}}_{a}\left(\underline{\underline{W_{2}}}+\mathbf{1}\right)^{-1}
$$

$\underline{\underline{W_{2}}}$ is the perturbation tensor from equation (2.62), without the elements $z_{H}$ and $z_{D}$

The result arrived at in equation (4.7) shows that we can use the magnetic field measured at a site $\mathrm{A}$ to do magnetotellurics at a site B if we know the Schmucker tensor, $\underline{\mathrm{W}}$ between the two sites. The term $\left(W_{2}+\mathbf{1}\right)^{-1}$ is the correction due to the anomalous field. It is expected that within the limit of experimental space, the impedance tensor $\underline{Z}$ from equation (4.1) should be equal to the impedance, $\underline{\underline{Z}}$.

The substitution is possible because the normal field is fairly uniform over the observation domain and varies quasilinearly over distances comparable to the threshold field penetration depth (Dmitriev and Berdichevsky, 1979, 2002)

\subsection{Error estimations}

For error estimations, we used an equation developed by Taylor (2005), for the calculation of errors in a function which involves the same quantity more than once. This equation is as follows: 
Suppose that $\mathrm{x}, \ldots, \mathrm{z}$ are measured with uncertainties $\delta x, \ldots, \delta z$ and the measured values are used to compute a function $\mathrm{p}(\mathrm{x}, \ldots, \mathrm{z})$. If the uncertainty in $\mathrm{x} \ldots, \mathrm{z}$ are independent and random, then the uncertainty in $\mathrm{p}$ is

$$
\delta p=\sqrt{\left(\frac{\partial p}{\partial x} \delta x\right)^{2}+\ldots+\left(\frac{\partial p}{\partial z} \delta z\right)^{2}}
$$

We applied equation (4.8) to our case as follows. For the seek of brevity, we illustrate only for the first impedance tensor element, $Z_{x x}^{\prime}$. From equation (4.7), the first impedance tensor element is given by

$$
Z_{x x}^{\prime}=\frac{Z_{a x x} w_{y y}+Z_{a x x}-Z_{a x y} w_{y x}}{w_{x x} w_{y y}+w_{y y}+w_{x x}+1-w_{y x} w x y}=\frac{Z_{a x x} w_{y y}+Z_{a x x}-Z_{a x y} w_{y x}}{D}
$$

where $D=w_{x x} w_{y y}+w_{y y}+w_{x x}+1-w_{y x} w_{x y}$

$$
\begin{aligned}
\frac{\partial Z_{x x}^{\prime}}{\partial Z_{a x x}} & =\frac{w_{y y}+1}{D} \\
\frac{\partial Z_{x x}^{\prime}}{\partial w_{x x}} & =\frac{-\left(Z_{a x x} w_{y y}+Z_{a x x}-Z_{a x y} w_{y x}\right)\left(w_{y y}+1\right)}{D^{2}} \\
\frac{\partial Z_{x x}^{\prime}}{\partial w_{y y}} & =\frac{D Z_{a x x}-\left(Z_{a x x} w_{y y}+Z_{a x x}-Z_{a x y} w_{y x}\right)\left(w_{x x}+1\right)}{D^{2}} \\
\frac{\partial Z_{x x}^{\prime}}{\partial w_{y x}} & =\frac{Z_{a x x}\left(w_{y y}+1\right)-\left(Z_{a x y} w_{y x}\right)\left(w_{x y}\right)-D Z_{a x y}}{D^{2}} \\
\frac{\partial Z_{x x}^{\prime}}{\partial Z_{a y y}} & =0 \\
\frac{\partial Z_{x x}^{\prime}}{\partial Z_{a x y}} & =\frac{-w_{y x}}{D} \\
\frac{\partial Z_{x x}^{\prime}}{\partial w_{x y}} & =\frac{\left(Z_{a x x} w_{y y}+Z_{a x x}-Z_{a x y} w_{y x}\right)\left(w_{y x}\right)}{D^{2}}
\end{aligned}
$$

By substituting the values from equations (4.10) to (4.16) and the corresponding values of $\delta Z_{a x x}, \delta w_{x x}, \delta w_{y y}, \delta w_{y x}, \delta Z_{a y y}, \delta Z_{a x y}$ and $\delta w_{x y}$ in equation (4.8), we obtain the error of the first impedance tensor, $Z_{x x}^{\prime}$. A semilar calculation is done for the other tensor elements $Z_{x y}^{\prime}, Z_{y x}^{\prime}$ and $Z_{y y}^{\prime}$.

\subsubsection{Results}

We first demonstrate our technique using two sites - EUL and DIA (see Figure 3.2) - where the full MT time series are available. This allows us to compare the transfer functions obtained using substituted magnetic field components against those obtained using the complete single-site time series. GDS transfer functions between DIA and EUL are shown in Figure 4.1. 
a)

b)

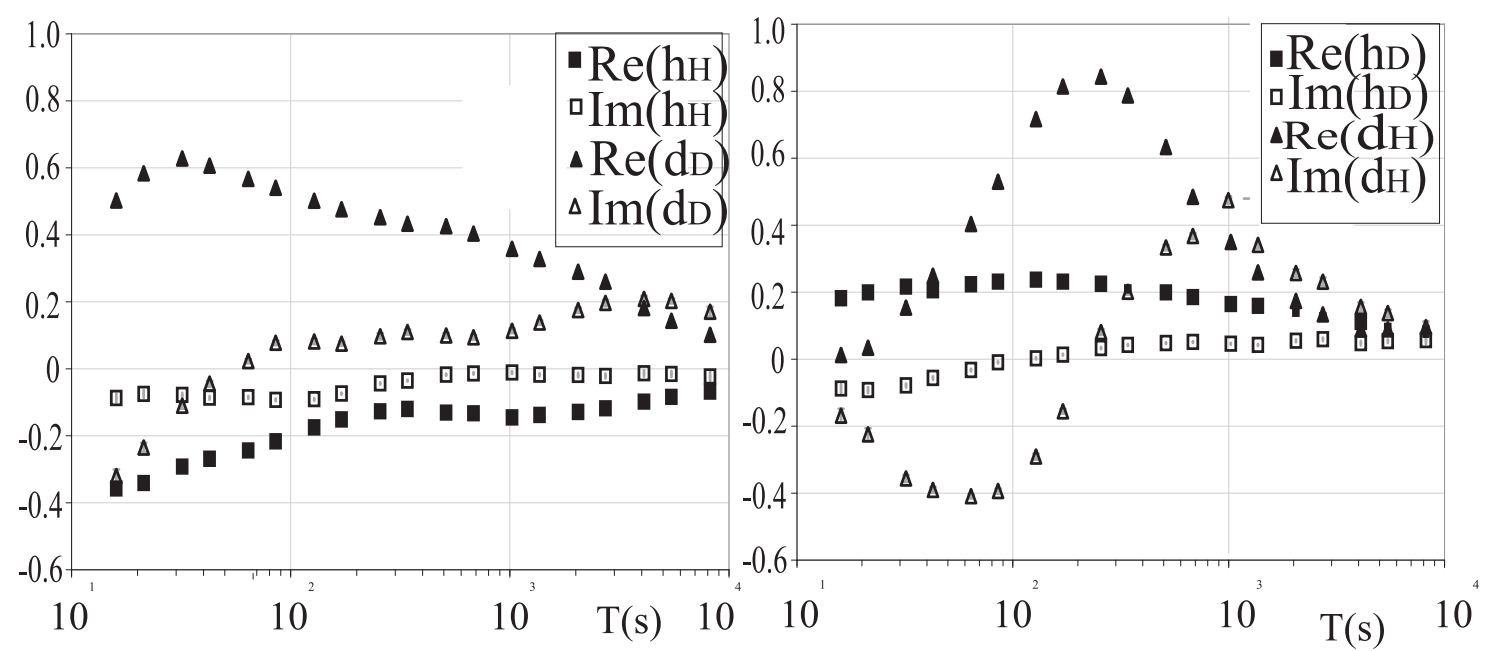

Figure 4.1: GDS transfer functions between EUL and DIA: a) principal diagonal elements of $\underline{\underline{W}}$; b) minor diagonal elements of $\underline{\underline{W}}$. The symbols used to plot points are larger than error bars. The sites are approximately $250 \mathrm{~km}$ apart (see Figure 3.2)

The magnetic anomaly that is apparent in these data can be attributed to a significant crustal conductivity anomaly previously identified by Chamalaun et al. (1999) and which will also be discussed in this thesis (section 5.3.3). The results of our processing are presented in figure 4.2 . 
a)

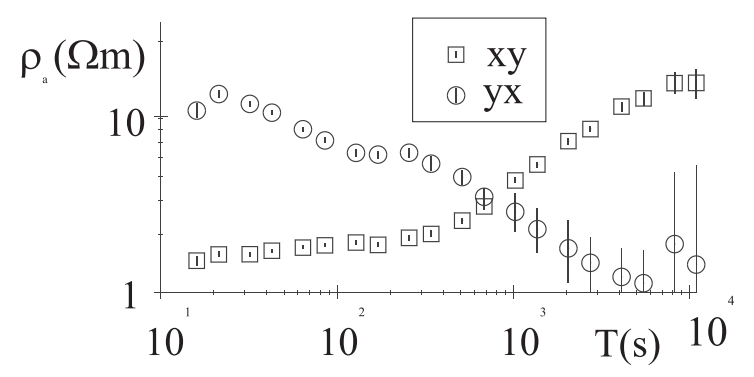

b)
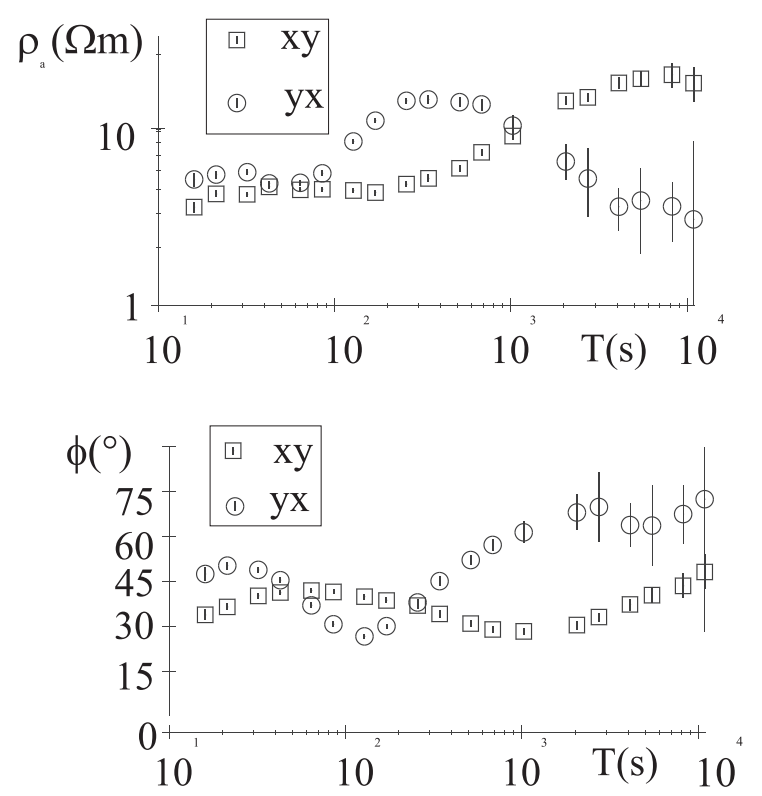

c)
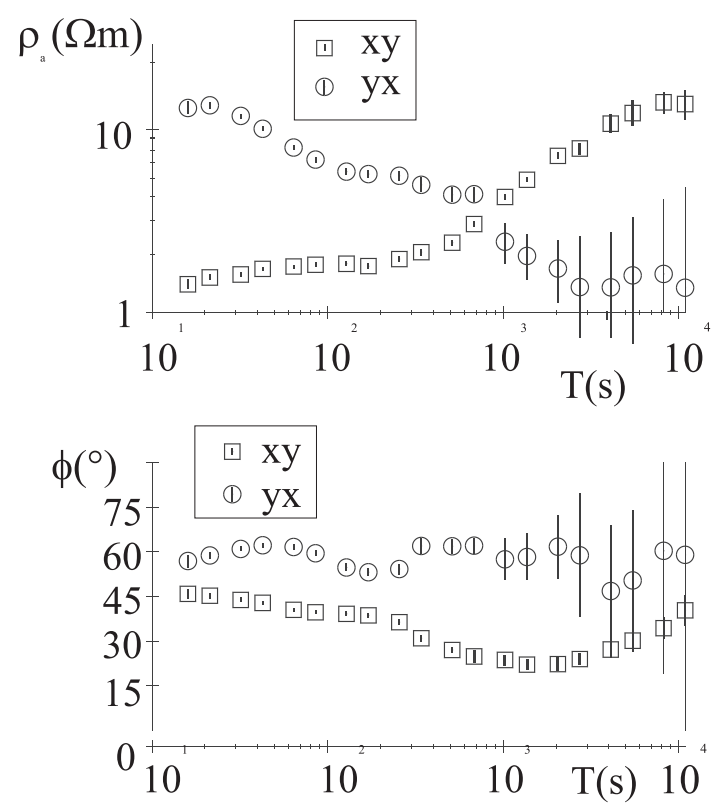

Figure 4.2: Comparison between the MT transfer functions obtained at EUL using a) the complete MT time series from EUL; b) the horizontal magnetic field components from DIA, with no correction for the substitution; c) the horizontal magnetic field components from DIA, with the correction given in equation (4.7) 
As expected, the measured apparent resistivities and phases at EUL (figure 4.2a) are significantly modified when the horizontal magnetic field components from DIA are substituted for those from EUL (Figure 4.2b). Notwithstanding, when the correction given in equation (4.7) is applied, the original MT transfer functions are satisfactorily recovered (figure 4.2c). Notice also that figures $4.2 \mathrm{a}$ and $4.2 \mathrm{~b}$ are more similar at longer periods for which the horizontal magnetic field gradient between EUL and DIA diminishes (see figure 4.2). It should be noted that the example we have presented is an extreme case, with a significant magnetic field anomaly. The technique has also been applied to sites between which the horizontal magnetic gradient is less, but significant enough that a substitution of the magnetic field from a neighbouring site is not permissible without applying the correction given in equation (4.7). In this case, the original MT transfer functions were able to be recovered exactly.

Next the technique was applied to the problem site, COOL. At COOL, the telluric field components were recorded for 65 days; the magnetic field components for only 25 days. The advantage of our technique is that it allows us to make use of the full telluric field recorded. We used data from TAMB (figure 3.2) to calculate $\underline{\underline{W}}$. The horizontal magnetic field gradients between COOL and TAMB are less than between EUL and DIA. Using this technique, the long period MT transfer functions are improved (Figure 4.3b) compared to those obtained from the data at COOL only (figure 4.3a).

a)

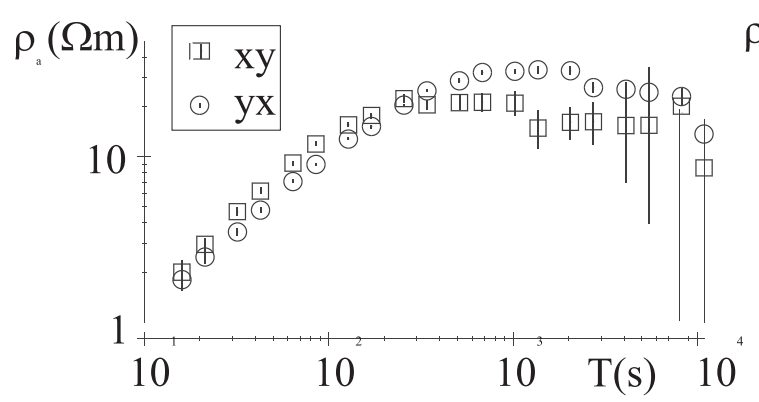

b)

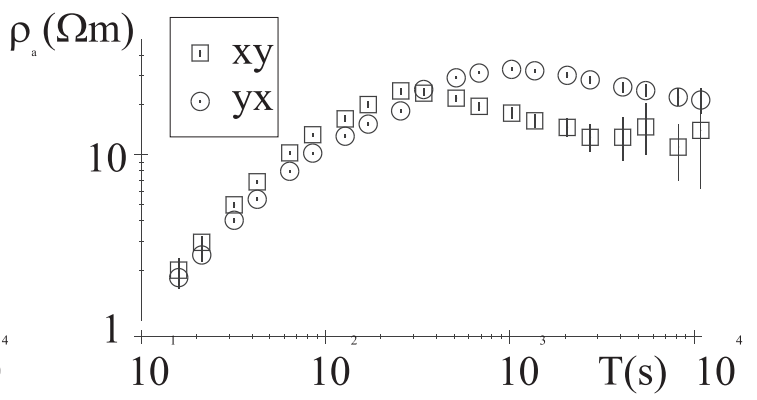

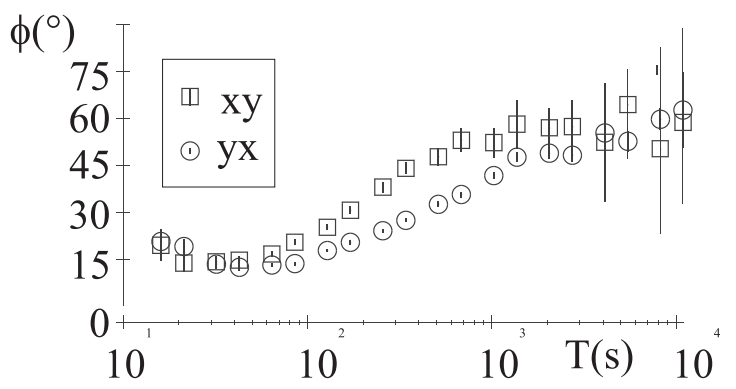

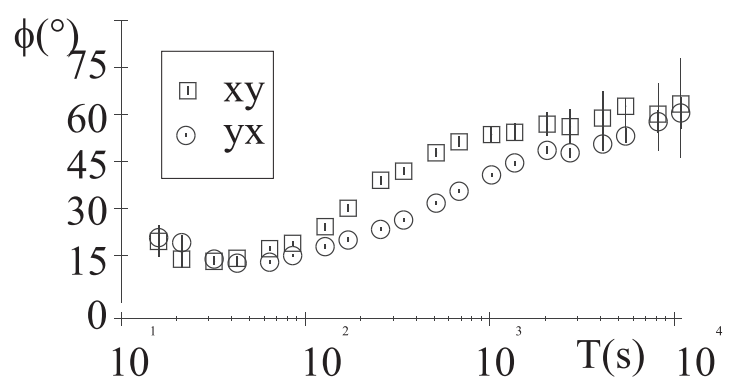

Figure 4.3: MT transfer functions for COOL using (a) limited time series recorded at COOL; (b) substituted horizontal magnetic fields from TAMB and the correction given in equation (4.7) 


\subsubsection{Conclusion}

We have developed a technique for retrieving high-quality MT transfer functions in the case that the horizontal magnetic field components at a site are incompletely registered. The technique involves substituting the magnetic field components from a neighbouring site and correcting for horizontal magnetic gradients using Schmucker's perturbation tensor, $\underline{W}$. We have applied the technique to data from Australia, where MT transfer functions were already known, and a significant magnetic gradient exists. When the remote magnetic field components from DIA were substituted at a local site EUL, the MT transfer functions were significantly modified, but by applying the correction term, we were able to recover the original MT transfer functions. Subsequently, we applied the technique to the station COOL, where the magnetic time series are incomplete. Here we were able to improve the quality of the long period MT transfer functions. The improvement arises because our method allows us to use the complete 65-day telluric time series, instead of only the 25 -days for which 5-component time series are available.

\section{3 $\mathrm{MT}$ results}

MT transfer functions and their characteristics for the 2000 data can be found in Simpson (2001). Below we present, for 2003 and 2007, MT transfer functions (phase only) that are representative of different regions of the survey area. Complete (phase and apparent resistivity) MT transfer functions are presented in appendix B.2. The MT transfer functions presented, apparent resistivity or phase or both curves are in the measured coordinate system with the $\mathrm{x}$-axis oriented in $\mathrm{N}-\mathrm{S}$.

The MT transfer functions can be grouped into five regions based on their phases. Sites in each region show similar phase behaviour in both rotated and unrotated coordinate systems. Figure 4.1 shows the five different regions. 


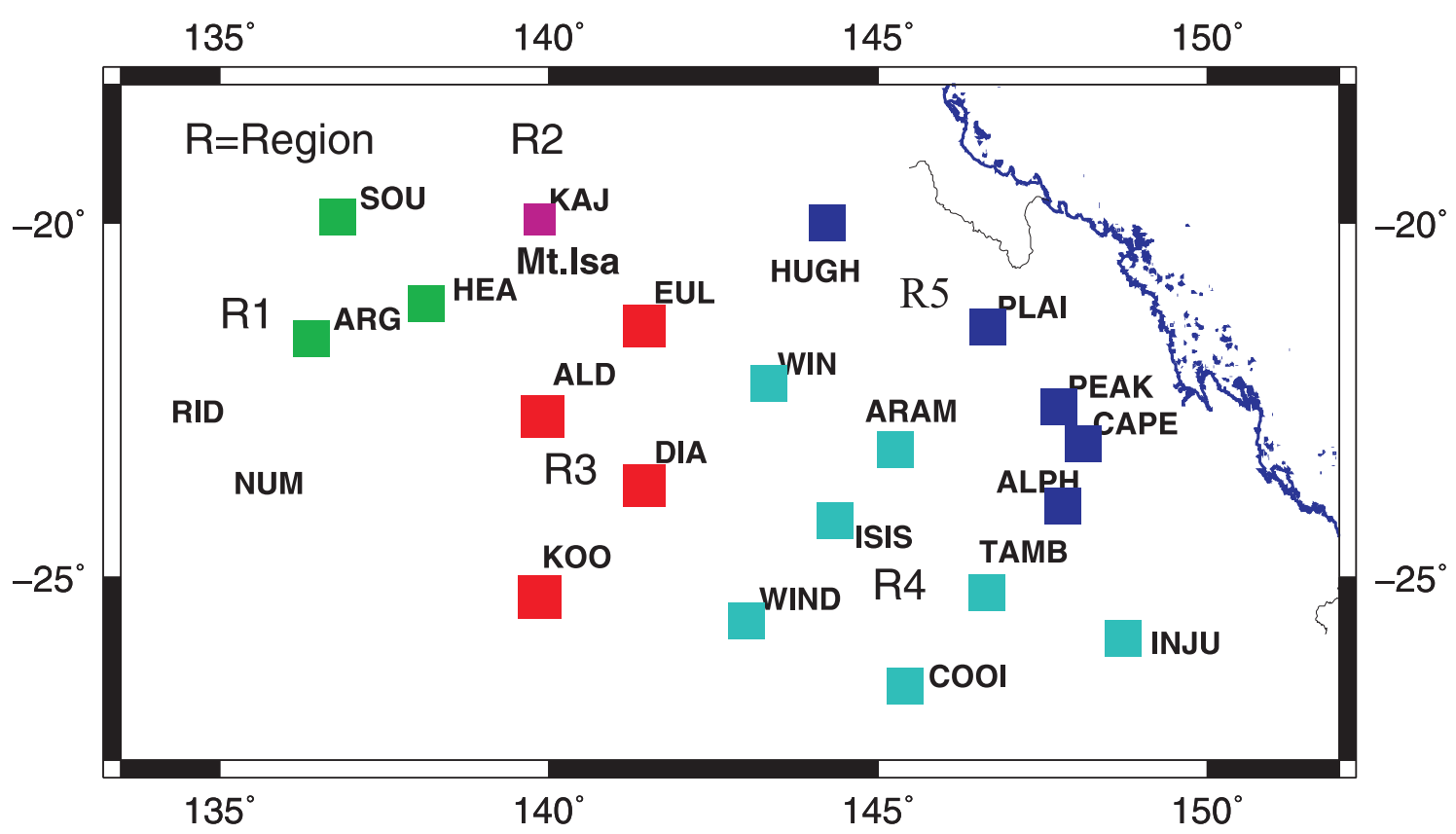

Figure 4.4: Survey area showing 5 regions shared according to phases of the MT transfer functions. Sites in each region have similar Phases in both rotated and unrotated coordinate systems

Region 1, denoted in figure 4.4 by dartmouth green squares lies between longitude $136^{\circ}$ $139^{\circ} \mathrm{E}$ and between latitude $-20^{\circ}-\left(-22^{\circ}\right) \mathrm{S}$. It comprised mainly of the sites ARG and HEA. The phase is large (about $62^{\circ}$ ) at shorter periods, decreases gradually to $45^{\circ}$ at $100 \mathrm{~s}$ and increases again at longer periods to a maximum of $60^{\circ}$. Its characteristic curves are near to that of a 1D structure (approximately equal phases in both polarisations) for periods below $250 \mathrm{~s}$ (upper crust). The phase curves of $\mathrm{E}$ (xy (red curve)) and B (yx)-polarisations (figure B.1) stay together while the apparent resistivities of corresponding polarisations move apart. This gives an indication of the effect of static shift or suggests that the region the hosts these sites is near a vertical contrast. In particular, site ARG shows a static shift effect over the whole period range indicated by a remarkable difference in resistivities for the two polarizations without any strong phase split (figure B.1). The fact that this region is affected by static shift means the presence of shallow conductivity structures since static shift is often caused by small scale conductive rather than resistive structures (Tournerie, Chouteau \& Marcotte, 2007). It is also important to note that apparent resistivities in this region are significantly larger than in the other regions of the surveyed area. Therefore strong small-scale conductivity gradients are more likely present in this resistive part of the array than in the more conductive eastern part in regions 2 and 3.

Region 2 represented in figure 4.4 above by a dark maggenta square lies between longitude $139^{\circ}-140^{\circ} \mathrm{E}$ and between latitude $-18^{\circ}-\left(-20^{\circ}\right) \mathrm{S}$. The site KAJ falls in this region. The phase is about $74^{\circ}$ between $8 \mathrm{~s}$ and $100 \mathrm{~s}$ and suddenly there is a large phase split at longer periods. This shows that in this region, at shorter periods (below $100 \mathrm{~s}$ ), the conductivity structure is 1D with resistivity decreasing with depth (high phase), high resistive upper crust followed by a more conductive lower crust. Above $100 \mathrm{~s}$ the large 
phase split (strong difference between the phase of the two polarisation) strongly suggests that the conductivity structure is no longer 1D but likely to be $3 \mathrm{D}$ (to be confirmed in the next section). The characteristics of the transfer functions at period above 100 seconds are similar to those in region 3 indicating that the anomaly in region 3 extends to this region at longer periods. The only difference that separates this region from region 3 is in the crust. Its crust is highly resistive. At longer periods (above $100 \mathrm{~s}$ ), regions 2 and 3 become the same and can be merged into one region.

The phase of the yx (B) polarization is about $90^{\circ}$ at some periods; an indication of complex 3D structures (Lezaeta \& Haak, 2003) which can be created by (1) bended band like conductivity structures or anisotropic structures with different directions (Heise \& Pous, 2003), (2) multiple 2D structures with different strike directions (Weckmann et al., 2003) or (3) a fractal system of interconnected high conductive phases (fluids or graphite) (Bahr, 1997). A complete analysis and interpretation of phases greater than $90^{\circ}$ can be found in Lilley and Weaver (2009).

The small phase value of the $\mathrm{xy}(\mathrm{E})$ polarization especially at longer periods is also common to region 3 that hosts the Carpentaria conductivity anomaly. The large phase in this region extends westward to region 1 . These effects can generally be explained in relation to the Carpentaria conductivity anomaly. The Mt. Isa Block falls in this region. Figure 4.5 below shows characteristic transfer functions in regions 1 and 2 . 


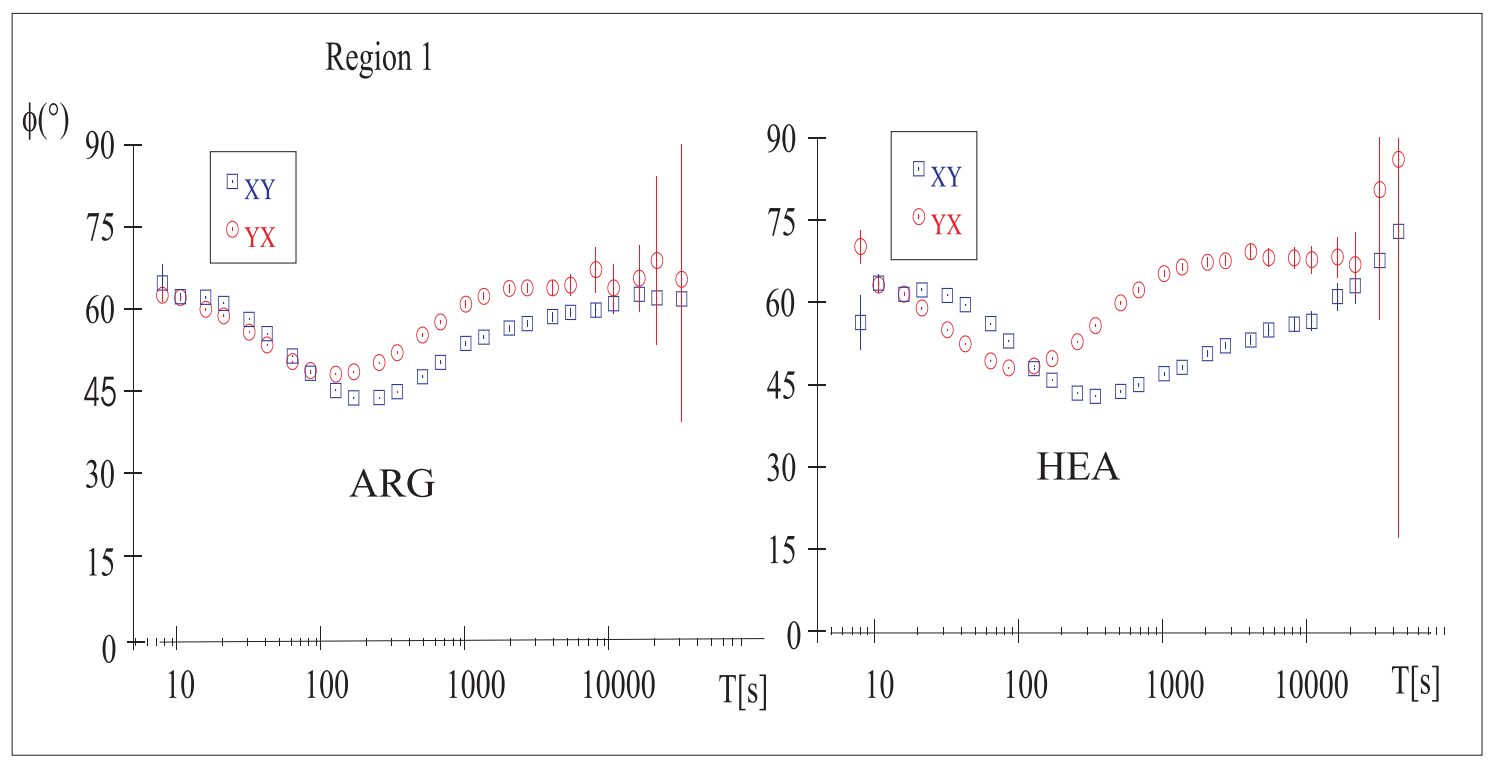

Region 2

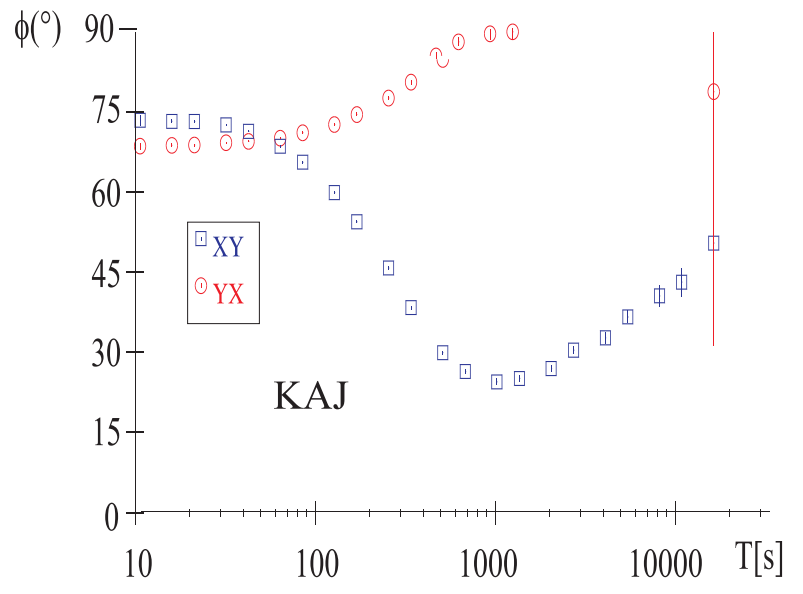

Figure 4.5: MT transfer functions (phases only) in an unrotated coordinate frame in regions 1 and 2. xy (E-polarisation) is north-south and $\mathrm{yx}(\mathrm{B}-$ polarisation) is east-west. 
Region 3 (red squares in figure 4.4) has coordinates between $\left(139.5^{\circ} \mathrm{E},-23^{\circ} \mathrm{S}\right),\left(141^{\circ} \mathrm{E}\right.$, $\left.-20^{\circ} \mathrm{S}\right),\left(143.5^{\circ} \mathrm{E},-22.5^{\circ} \mathrm{S}\right)$ and $\left(139^{\circ} \mathrm{E},-25^{\circ} \mathrm{S}\right)$; it comprises the sites EUL and ALD. Sites WIN, DIA and KOO all belong to this region (large phase split at longer periods is their grouping criterion) and in region 4 (small phases at short periods-characteristic of the Eromanga sedimentary basin; that is at short periods, the effects of the conductive sediments overshadow that of the anomaly). The phase is about $45^{\circ}$ except at WIN, DIA and KOO- there is a strong phase split between the two polarization along the transect comprising regions 2 and 3 . A consistent phase split, coupled with a similar phaseddefined resistivity strike over a large array is often cited as evidence for anisotropy in the lithosphere and/or asthenosphere if there is no accompanying vertical field (Mareschal et al., 1995; Simpson, 2001; kellett et al., 1992). But along this transect (figure 4.4), the vertical field exists (to be proved later under GDS analysis, section 4.4). Site ALD has much larger values of apparent resistivities at shorter periods than the other sites in this region (figure B.2); it is out of the conductive sediments of the Eromanga Basin whose sites are characterised by small resistivities values at the uppermost zones. For all sites in the region, the transfer function curves show a strong indication of an anomaly and the large phase split is a clear indication that the conductivity structure in this region cannot be $1 \mathrm{D}$. This is the region that hosts the Carpentaria conductivity anomaly and through which the Tasman Line is delineated, east of KAJ through EUL to KOO. Figure 4.6 gives a picture of the nature of the MT transfer functions in the region. 

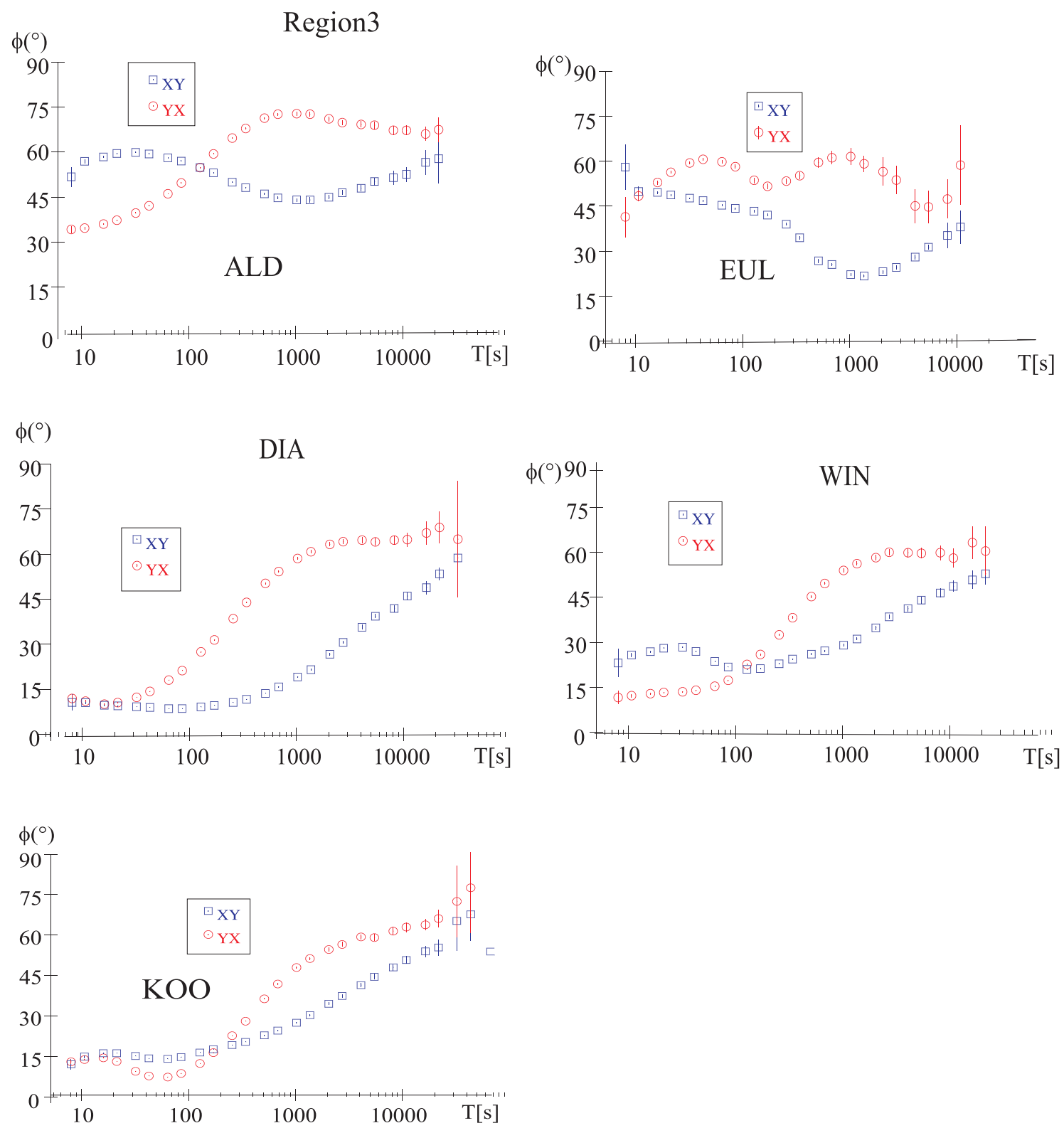

Figure 4.6: Unrotated MT transfer functions (phase curves only); representative of region 3. 
Between the coordinates $\left(139^{\circ} \mathrm{E},-23^{\circ} \mathrm{S}\right),\left(143^{\circ} \mathrm{E},-22^{\circ} \mathrm{S}\right),\left(145.3^{\circ} \mathrm{E},-22.5^{\circ} \mathrm{S}\right),\left(146.4^{\circ} \mathrm{E},-\right.$ $\left.24.5^{\circ} \mathrm{S}\right)$ and $\left(148.6^{\circ} \mathrm{E},-26.7^{\circ} \mathrm{S}\right)$ is region 4 represented in figure 4.4 by deep sky blue squares. The sites in this region include KOOL, WIND, WIN, ARAM, ISIS, TAMB, COOL and INJU. They have a phase of at least $15^{\circ}$ at short periods and which increases to about $60^{\circ}$ at longest periods. The small phases at short periods characterise the conductive sediments of this region (the Eromanga Basin) that are underlain by more resistive rocks. Like in region 1, some sites in this part of the array, for example ARAM, are affected by static shift (figure B.3). However, in general, sites in this region have reduced static shift effect. In practice the amount of static shift that is observed in sedimentary areas is generally much smaller than that observed in crystalline areas (Sternberg et al., 1988 and Rasmussen, 1999). This is because there are more near surface inhomogeneities with higher conductivity contrasts in crystalline areas (Zhang et al., 1987, 1992) than in sedimentary environments. Note the intersection between regions 3 and 4 if one examines the phases at short periods. The nature of MT transfer functions in this region is given in figure 4.7 below. 
Region4
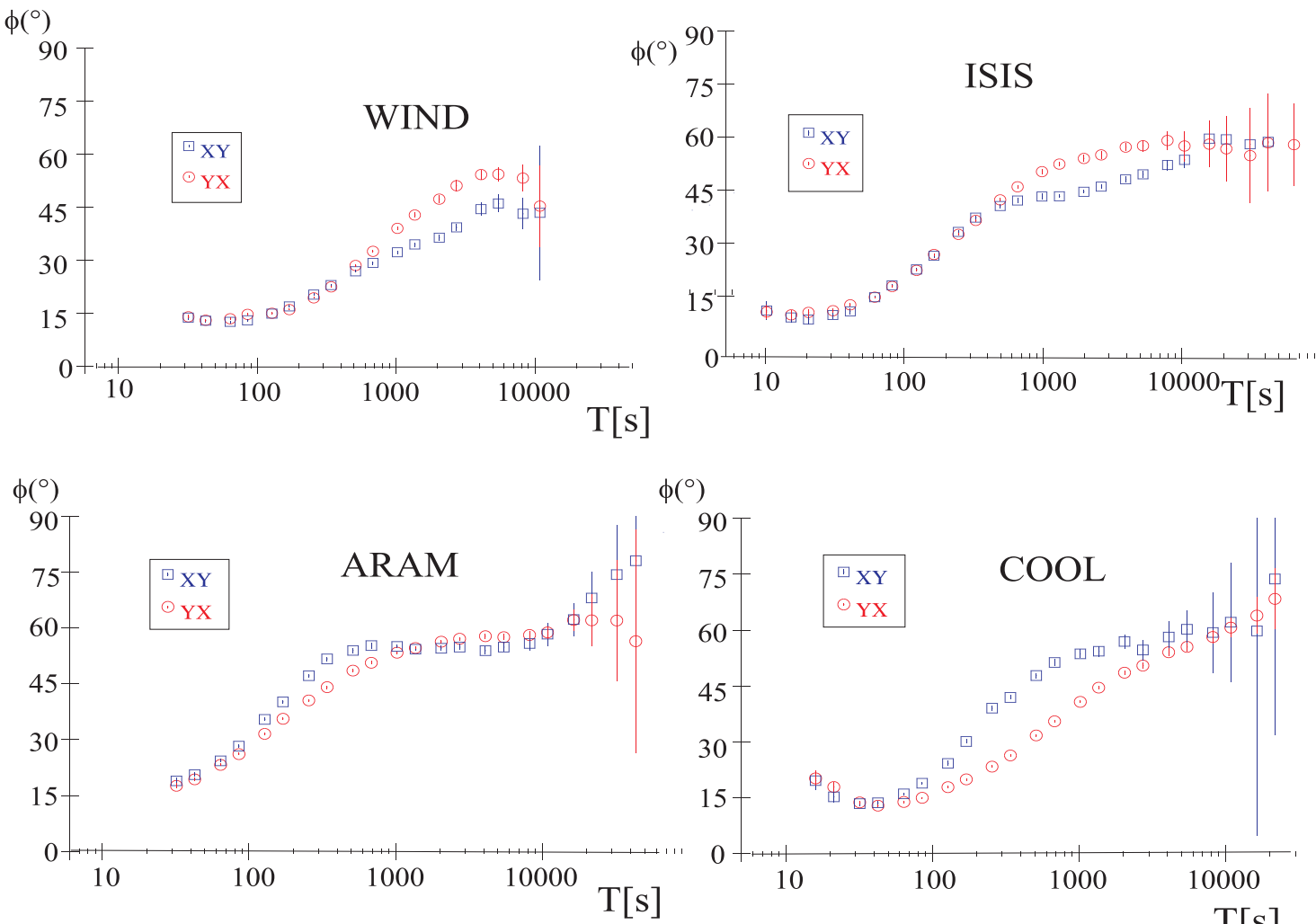

$\phi\left(^{\circ}\right)$
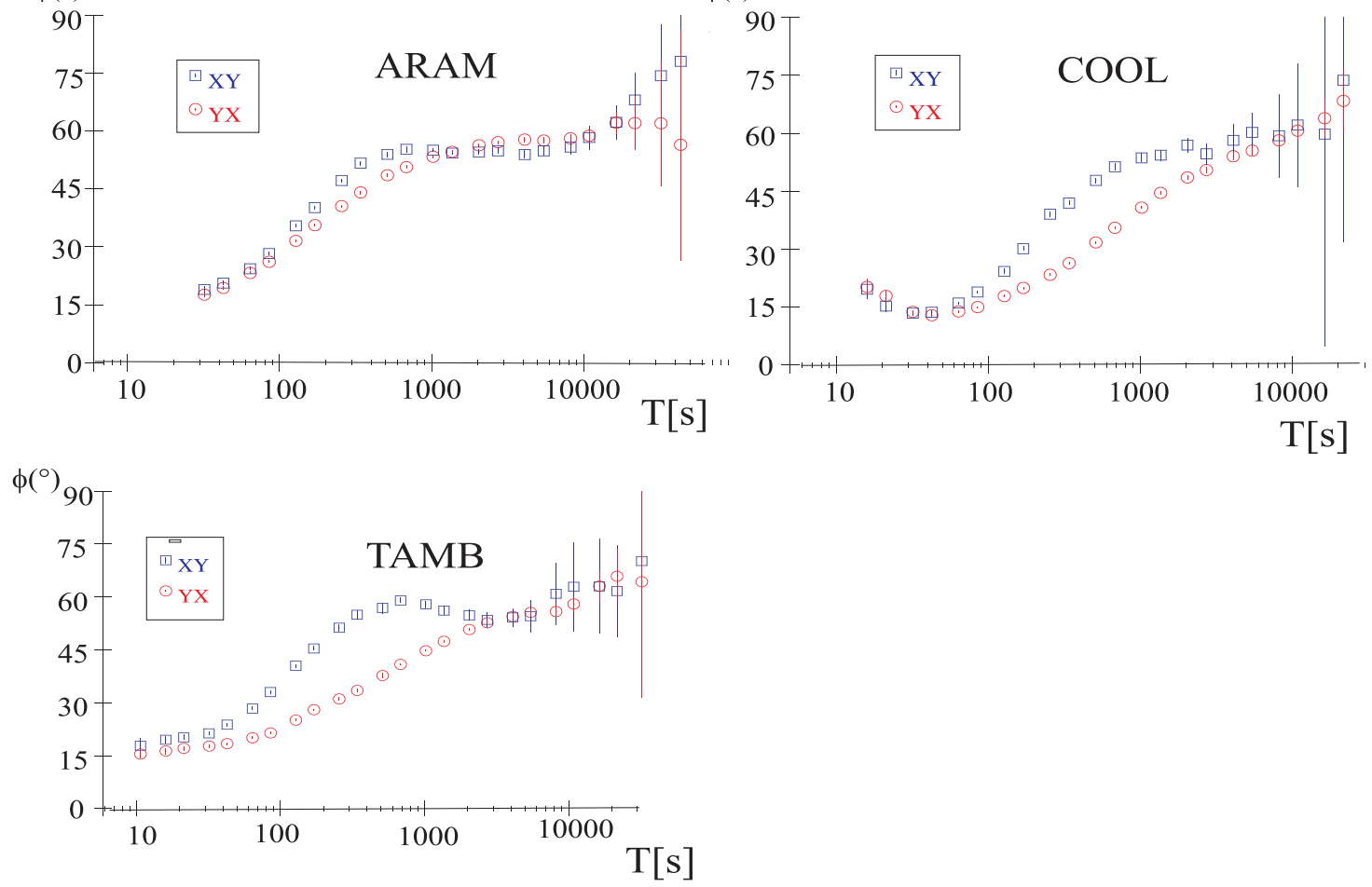

Figure 4.7: Unrotated MT transfer functions (phase curves only); representative of region 4 . 
Region 5 (dark powder blue squares in figure 4.4) comprises the sites HUGH, CAPE, PEAK, and PLAI. It is northeast of the sedimentary basin and lies between $\left(144^{\circ} \mathrm{E}\right.$, $\left.20^{\circ} \mathrm{S}\right)$ and $\left(148.1^{\circ} \mathrm{E},-23.1^{\circ} \mathrm{S}\right)$. Sites in this region are closer to the ocean than other sites in the survey area and are likely to be affected at longer periods by a coast effect (section 3.4). The phase of the MT transfer functions is about $45^{\circ}$ with a strong split between the two polarisations, especially at short periods. The characteristic transfer functions of this region predict it to be $2 \mathrm{D}$ or $3 \mathrm{D}$ especially between the crust and upper mantle. See figure 4.8 for its characteristic transfer functions
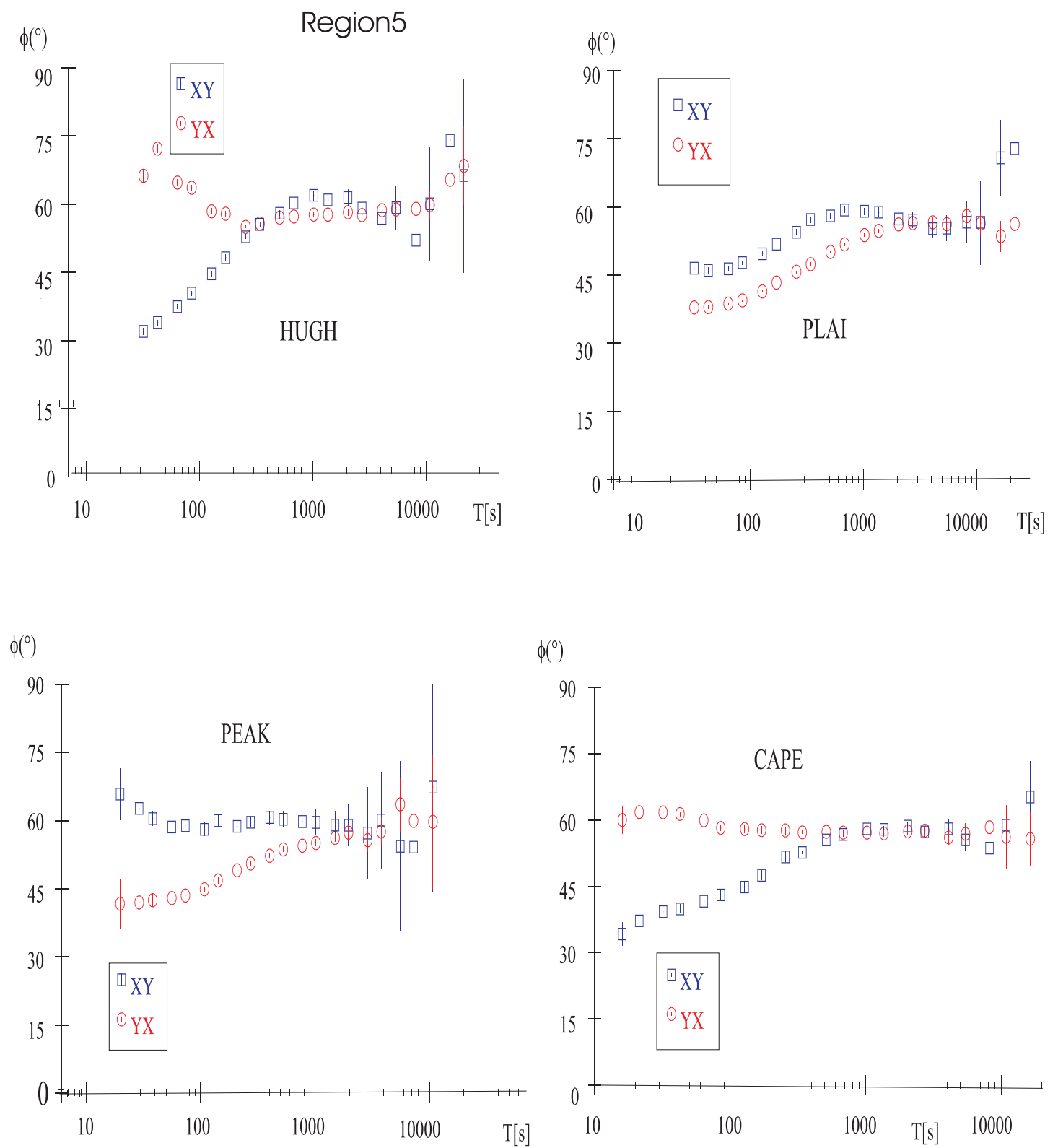

Figure 4.8: Unrotated MT transfer functions (phases only); representative of region 5 .

In all the regions of the array, apparent resistivities and especially the phases between 
neighbouring sites are well correlated. This means the main conductivity structures in the survey area have a greater extent than the site spacing of about $100 \mathrm{~km}$; they are therefore resolvable by our measurements.

\subsubsection{Induction vectors}

Figure 4.9 shows local ${ }^{14}$ induction vectors for a period range between $32-10870$ seconds. At short periods, below $43 \mathrm{~s}$, the pattern is not clearly uniform for all sites. For sites in region 5 defined (figure 4.4), the induction arrows are a bit random. For example, for some sites, induction vectors are pointing northwest while others are pointing northeast. This indicates the influence of small scale structures (local shallow 3D conductivity structures) leading to distortion in this region that are irresolvable since the station spacing is not sufficiently dense to delineate the finer-scale patterns in detail. In addition, in this period range, the induction vector of site ALD in region 3 is pointing approximately north. This can only be explained in terms of small scales conductivity structures. However, the northsouth conductor (Carpentaria anomaly, Chamalaun et al. (1999)) running through sites EUL and DIA is already clearly visible at these periods, that is real induction vectors of sites between longitudes $140^{\circ}$ and $143^{\circ} \mathrm{E}$ are clearly pointing in opposite directions (away from the Carpentaria anomaly, see figure 4.10).

In this period range, the coastline effect to the east of the array is not yet observable. This should be understood since the sea is more than $500 \mathrm{~km}$ from the nearest site CAPE, to the East. That means these periods cannot see the effect of the conducting Coral Sea in the east.

For periods above $128 \mathrm{~s}$, the coastline effect, judging from the direction of the induction vectors, becomes conspicuous. All real induction vectors of sites to the extreme east of the array are pointing to the west; opposite to the conducting Ocean (figure 4.10 simplified this description). The west pointing arrows at PLAI, PEAK and CAPE represent the conventional geomagnetic coast line effect related to the concentration of induced currents in the conducting Coral Sea water (Electrical currents flow mostly in the sea, resulting in large vertical fields at the coastal area (Honkura, 1978)). This influence of such oceanic currents is found to increase at increasingly longer periods. For example, in figure 4.9, the real induction arrows at stations PLAI, PEAK and CAPE in the eastern part of the array, swing from their eastward directions at 32 and $43 \mathrm{~s}$ to a westward direction (away from the coastline), at $128 \mathrm{~s}$ and above. Therefore, since these stations are more than 500 $\mathrm{km}$ from the sea, at longer periods, the effect of the coastline is discernible at distances greater than $500 \mathrm{~km}$. This does not come as a surprise, it only complement studies of the coast effect by other authors; Kellet et al. (1988), in their study observed that the southeast Australian coastline causes anomalous vertical magnetic field (Z) fluctuations which extend to at least several hundred kilometres from the coast. Similar results were noted by Chamalaun et al. (1999). These results are in accordance with the fact that induction arrows are often influenced significantly by regional conductivity anomalies that lie outside the MT model space.

${ }^{14}$ parochial, at a particular site, not regional or global for the study area 
The period range of maximum induction indicated by maximum amplitude of the real induction arrows (section 2.3.2) is between 256 and $512 \mathrm{~s}$ for most sites in the study area, especially for sites west of longitude $142.5^{\circ} \mathrm{E}$ where their amplitude of real induction arrows have not been cancelled by the coast effect. 
Induction arrows

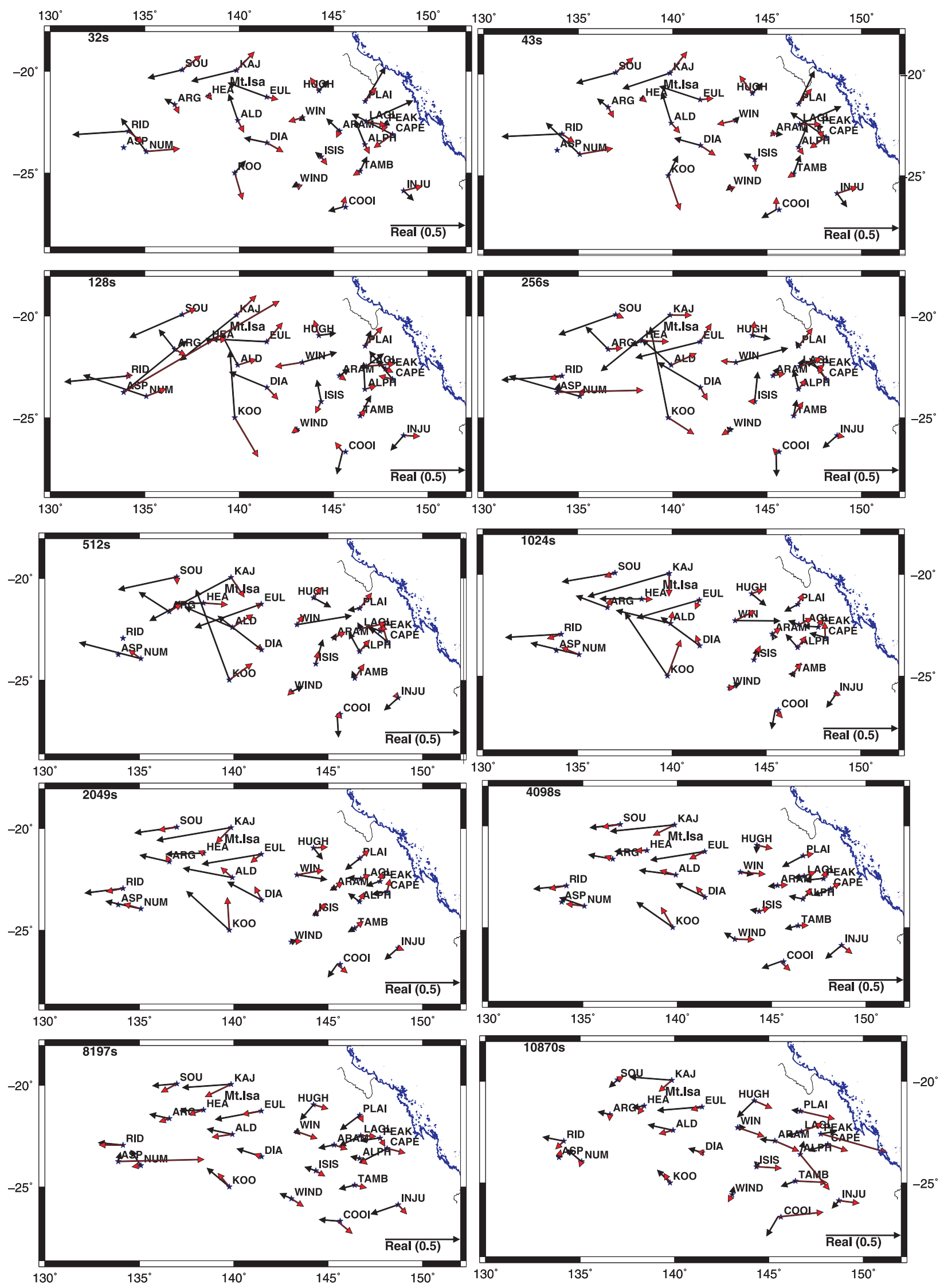

Figure 4.9: Real (black) and imaginary (red) local induction arrows for 10 periods. 85 
Given that to the north of our project area, we also have some sites e.g. KAJ close to the Gulf of Carpentaria, how do we explain the absence of a coast effect to the north? The answer is simple; the Gulf of Carpentaria is relatively shallow (it is everywhere less than $70 \mathrm{~m}$ deep (Peter et al., 2004); "the Gulf of Carpentaria is an epicontinental sea (maximum depth $70 \mathrm{~m}$ ) between Australia and New Guinea, bordered to the east by Torres Strait (currently $12 \mathrm{~m}$ deep) and to the west by the Arafura Sill $(53 \mathrm{~m}$ below present sea level)" Chivas et al., 2001). In contrast to the Coral Sea, the Gulf is an insufficient electrical conductor to cause a coastline effect at long periods. Hence, our results agree with previous results that the sea in the Gulf of Carpentaria is shallow and therefore not large enough to support extensive eddy currents.

Also at longer periods the induction vectors take a pattern that can be explained. For example, at $128 \mathrm{~s}$, the strike direction of the conductor (Carpentaria anomaly, figures 3.4 and 3.5 and the stipulated Tasman Line (note that in North Queensland, the Carpentaria anomaly and the conductor defining the stipulated Taman Line overlapped)) through sites EUL and DIA is north -south. This is so because the real part of the induction vectors at sites KAJ, EUL, ALD and DIA on one site and HUGH and WIN on the other site are pointing in opposite directions, west and east respectively and are perpendicular to the north-south direction (strike direction), see figure 4.10. As the period increases, say to $256 \mathrm{~s}$, the real induction vectors of sites close to and on opposite site of the conductor are slightly no longer perpendicular to the north-south strike direction. This means that the strike direction is changing slightly at longer periods and is no longer exactly north-south. However, at a period of $1024 \mathrm{~s}$ and above, between latitude $22^{\circ}$ and $22.5^{\circ} \mathrm{S}$, the strike direction is exactly north-south; see the direction of induction vectors of sites ALD and WIN at $1024 \mathrm{~s}$ and above. Their directions are opposite and exactly perpendicular to the north-south direction.

The direction of the induction vectors of sites in the east of the survey area can best be explained using the site WIN (nearest to the Carpentaria anomaly and east of it) and PLAI (also east of the anomaly but further from it and closest to the Coral Sea). The real induction vector at WIN is pointing to the east, that is opposite to the Carpentaria anomaly while that at PLAI is pointing to the west, influenced by the conducting Coral Sea to the east. That is induction vectors at PLAI are pointing to west, opposite to the conducting sea to the East.

The induction vectors have their maximum length between $300 \mathrm{~s}$ and $1024 \mathrm{~s}$ (which I may now term the extended period range of maximum induction, especially for sites close to the Carpentaria anomaly/our stipulated Tasman Line). This is the period range at which the conductive effect of the anomaly is very strong. As we will see later in the next section, this is in agreement with the high amplitude of the $d_{D}$ perturbation tensor at this period range.

Another conspicuous feature of the pattern of induction vectors is that sites close to and west of the Carpentaria anomaly/the anomaly that marks our stipulated Tasman Line have longer induction vectors than those to the East. This is because sites to the east are sandwiched between the anomaly and the conducting sea. In this case the effect of the conducting seawater tries to cancel out that of the anomaly. But since the anomalous Z-field of the conductor is stronger and has an opposite sign compared to the Z-field generated by the sea, sites to the east and closer to the conductor have a net vertical field 
caused by the Carpentaria conductor. Hence induction vectors of sites to the east have shorter length compared to those in the west. This is in perfect agreement with results published by Heise et al. (2006) that small induction vectors can occur where the vertical magnetic field components produced by lateral changes cancel out. In a classic paper by Honkura (1978), he also observed that the effect of an anomalous conductor below the Peruvian Andes is large enough to cancel the coast effect by anomalous Z-fields of opposite sign.

Looking at the pattern of induction vectors to the north of our array, it is obvious that the Carpentaria anomaly extends further north into the Gulf of Carpentaria as suggested by previous studies.

Sites to the West of the array have induction arrows with greater amplitude than those to the East and are pointing westward. Their induction vectors are influenced by a "band like conductor" dividing the Precambrian western and Phanerozoic eastern Australia between approximately longitudes $141^{\circ}$ and $142.5^{\circ} \mathrm{E}$ (figure 4.10 ). The pattern followed by the real induction arrows at these sites become vivid at longer periods starting, say at 1024 s. As already mentioned above, the small amplitude of real induction vectors at sites to the east compared with sites to the west of the array is due to the fact that these sites are sandwiched between the conducting ocean and the "band like conductor" " running along longitudes $141^{\circ}$ and $142.5^{\circ} \mathrm{E}$.

A careful examination of the induction vectors of site $\mathrm{KOO}$ at all periods reveals that they are independent of the north-south conductor (Carpentatia anomay) located at approximately longitude $141.5^{\circ} \mathrm{E}$. Observe that induction vectors of sites KOO and WIND at all periods are not perpendicular to the north-south direction. This indicates that the extent of this anomaly is overshadowed in the south after latitude $25^{\circ} \mathrm{S}$. The induction vectors of sites KOO and WIND are influenced by a different conductor south of them. This is also supported by the fact that the induction vector of site $\mathrm{KOO}$ is about 4 times greater than that of site WIND; an indication that its source is different from the N-S conductor through EUL and DIA. The conductor to the south must be closer to KOO than WIND. This conductor is most likely to be an extension in the north direction of the southwest Queensland/Arckaringa anomaly (Gough et al., 1974 and Chmalaun \& Barton, 1993). Its nature and extent cannot be determined by this study since no sites south of KOO were measured.

Figure 4.10 below summarises the description above about induction arrow directions (patterns) and the conductors that influence them. A in the figure represents the Carpentaria anomaly and the band like conductor that marks the that Tasman Line. B is the southwest Queensland anomaly. The influence of the Coral Sea is also represented on the top right. 


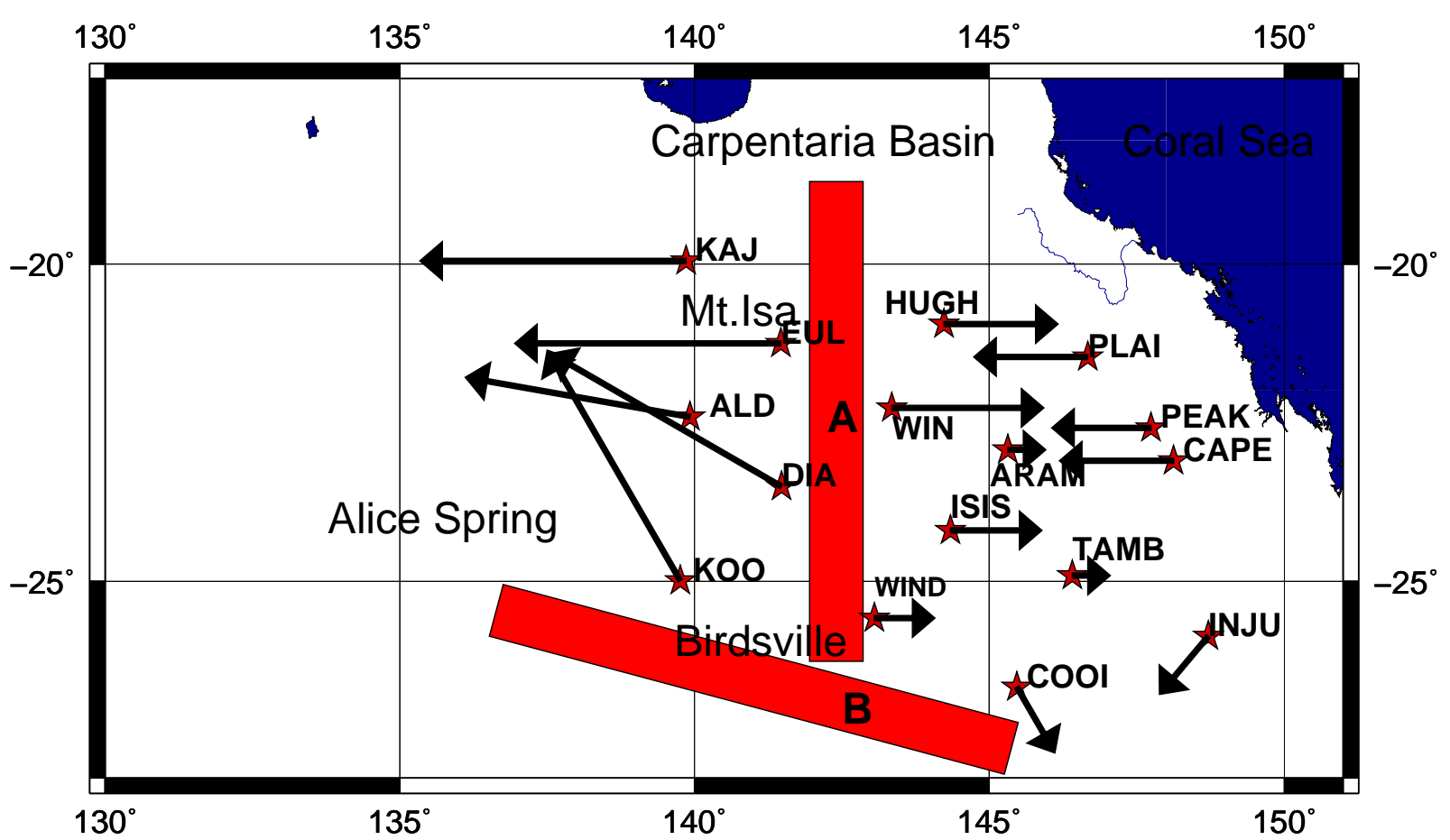

Figure 4.10: Sketch of major anomalies with respect to stations showing how these anomalies influence induction arrow pattern. $\mathrm{A}$ is the Carpentaria anomaly that overlaps with the Taman Line. B is the southwest Queensland Anomaly.

From the induction vectors alone, it is difficult to draw any conclusion about the idea of the Tasman Line. However, the north-south trend of the line at longitude $142.5^{\circ} \mathrm{E}$, between latitudes $18^{\circ}$ and $25^{\circ} \mathrm{S}$ is already visible. A detailed model is needed to draw a conclusion.

\subsubsection{Strike direction and phase sensitive skews}

To determine the electromagnetic strike at each site, we used the superposition model of Bahr(1988) (equation 2.53). In this model, the electromagnetic strike at each site is estimated by finding the direction that maximizes the splitting of the impedance phases. Figure 4.11 below is a plot of electromagnetic strike at the measured sites for different periods. 
Strike angles

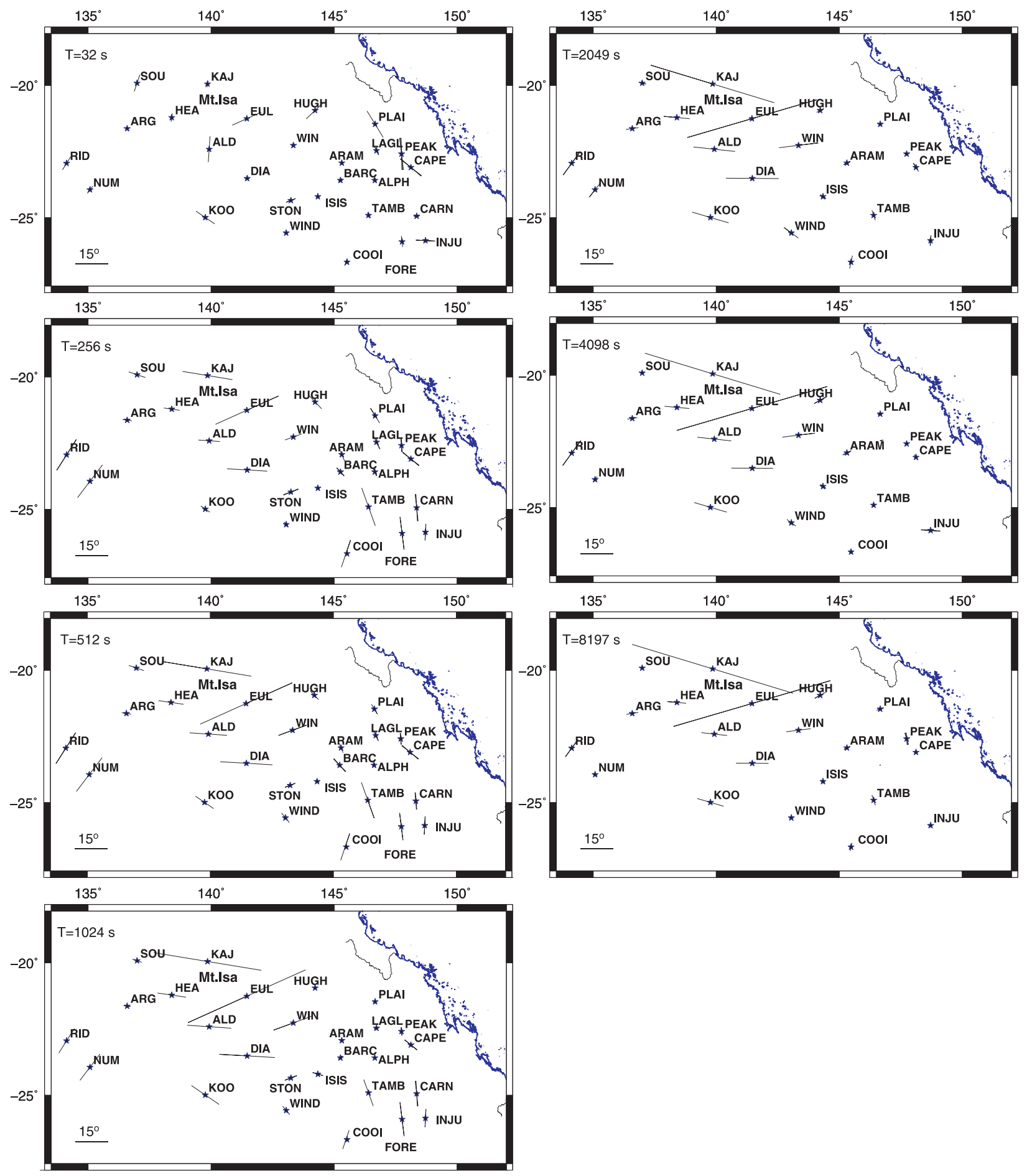

Figure 4.11: The line through each station (star) shows the electromagnetic strike for a given period. The length of the bars indicates the amount of phase splitting $\phi_{x y}-\phi_{y x}$. 
At short periods, for example $32 \mathrm{~s}$, the phase difference for most sites is small and the strike directions meaningless since they may be related to small scale structures that can not be resolved by the site spacing in our array. In particular, in the conductive sediments of the Eromanga Basin (sites WIN, DIA, KOO, ISIS, WIND, TAMB, STON, COOL, INJU and FORE in figure 4.11 fall in the Eromanga Basin ), the phase difference is very small due to uniform conductivity of the sediments. Therefore one cannot identify a strike direction at short periods in the Eromanga basin. An exception is at site KOO whose strike may be influenced by the southwest Queensland conductivity anomaly even at short periods. In this period range, an approximate north-south strike direction can be identified for sites in region 5 (PLAI, PEAK, CAPE belong to this region) which are out of the Eromanga basin. In region 3 that includes ALD, EUL, DIA and KOO, the strike is north-south especially at ALD. This can be attributed to the predominant major fault zones in this region (Bierlein \& Betts, 2004).

From 256 seconds and above, the strike directions in the whole survey area become more coherent. The consistent strike angle means that the superposition model (section 2.3.3) is valid for the survey area. At a period of 512 seconds, the strike in the central part of the array is east-west except at the site EUL (site at the heart of the Carpentaria anomaly) that is $\mathrm{N} 45^{\circ} \mathrm{W}$. Southeast of the survey region, the strike is north-south. To the east and south of WIN, the phase split is negligible and the strike direction meaningless. The biggest phase split is observed at the site EUL that coincides with the position of the Carpentaria conductivity anomaly (Chamalaun et al., 1999). The trend continues up to a period of about 2000 seconds. Above 2000 s, a strike direction can only be identified in the Central part of the array between longitude $138^{\circ}$ and $142.5^{\circ} \mathrm{E}$. The phase split at sites EUL and KAJ increases with increasing period and have a maximum phase split of about $50^{\circ}$ between 4000 and 8200 seconds. For sites, east of the array, the phase difference become negligible and the strike direction meaningless. This means above 2000 $\mathrm{s}$ the eastern part of the array approximates to a $2 \mathrm{D}$ conductivity structure.

Changes in the amount of phase splitting from site to site indicate some lateral variation of conductance in the highly conductive direction; however, one observes that the direction itself is stable over large distances. The amount of phase split decreases with distance from the Carpentaria anomaly indicating that phase split in the area is essentially due to a laterally displaced conductivity anomaly rather than deep anisotropy. If phase splitting is due to deep anisotropy, the degree of impedance phase splitting will remain the same over larger distances (Bahr \& Simpson, 2002).

In general the strike directions do not concur well with the direction perpendicular to the induction vectors direction, especially along the north south Carpentaria anomaly. If the superposition model is valid, then it is difficult to explain at this stage the reason for this nonaccordant between the direction of the strike and that of the induction vectors. In section 2.4 (equations 2.78 and 2.79) we concluded that the geomagnetic strike is independent of magnetic distortion; we will therefore determine in the next section under GDS, the geomagnetic strike in order to have a clearer insight into the strike direction. 
The phase sensitive skew values

Phase sensitive skew

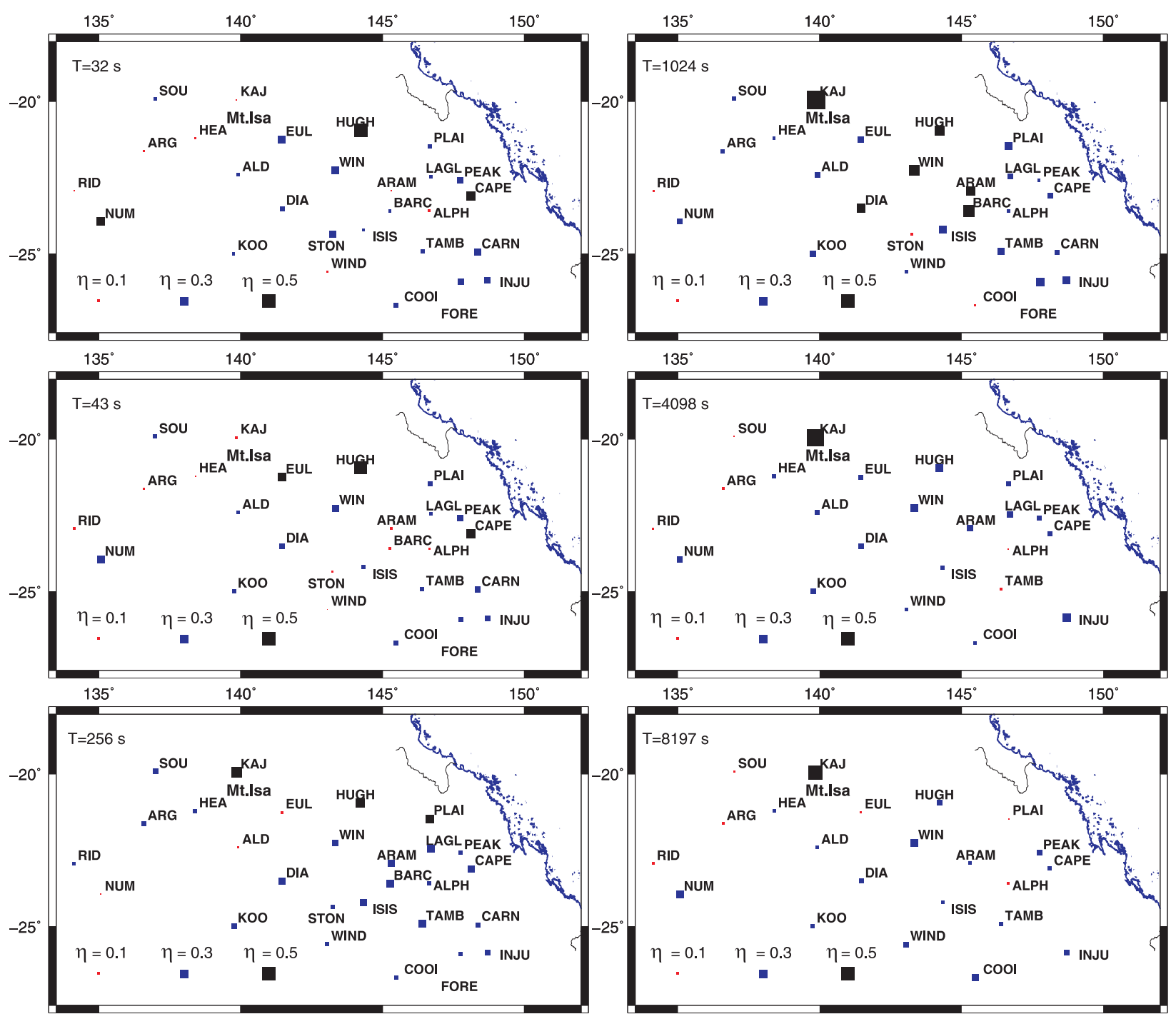

Figure 4.12: Phase sensitive skew values eta $(\eta)$. Colours classify the skew in 3 classes: red $(<0.1)$, blue $(0.1$ to 0.3$)$ and black $(>0.3)$.

In section 2.3.5 (equation 2.60), we introduced the phase sensitive skew parameter $(\eta)$ that can be used to characterize dimensionality. Figure 4.12 shows the values of $(\eta)$ for each site calculated at different periods. At short periods (e.g. at $32 \mathrm{~s}$ ), little conclusion can be drawn from the skew values. However, worthy to note is the extremely small skew value at site KAJ at this period range and the large value for site HUGH. The small skew value for site KAJ at short periods consent with our discussion in section 4.3 that since there is no phase split between the two polarisations ( $\mathrm{xy}$ and $\mathrm{yx}$ ) up to about $100 \mathrm{~s}$, the conductivity structure in and around KAJ is $1 \mathrm{D}$. We had also mentioned in the same section that 3D structures at the site indicated by a large phase of about $90^{\circ}$ orignate at long periods. This is confirmed here by large skew values at site KAJ starting at long periods only. The 
large skew values at short periods at site HUGH may indicate 3D conductivity structures in this period range. As the period increases to 256 seconds, some trend in skew values could be observed; skew values greater than 0.3 (black) are identified from KAJ through HUGH (between latitude $19.5^{\circ}$ and $21.5^{\circ} \mathrm{S}$ ) to PLAI. The skew values at EUL and ALD are very small (red) and do not reflect their transfer functions which suggest 3D structures. However, this is not too strange, since according to Bahr (1988), small skew values do not necessarily demand $1 \mathrm{D}$ or $2 \mathrm{D}$ structures.

At a period of 1024 seconds, the large skew values described above at 256 seconds extend south of HUGH to include DIA, WIN and ARAM. At this period, the effects of the uniform conductive sediments within this region decrease significantly and 3D conductive structures emerge. This is the region that hosts the Tasman Line as discussed in the introduction.

At longer periods (4098-8197)s, skew values become less than 0.3 for all sites except at site KAJ. The general trend is that skew values increase to a maximum at 1024 seconds where three dimensional effects are maximal (between longitude $140^{\circ}$ and $145^{\circ} \mathrm{E}$ ) and decrease for longer periods where distortion effects of the conductive block (Carpentaria anomaly) become less significant. As we will see later under GDS, the maximum skew values in the region at 1024 seconds are in agreement with the maximum amplitude of perturbation tensor at 1024 seconds.

The extremely large skew value (compared to other sites) at site KAJ is in agreement with our earlier conclusion that the Carpentaria anomaly extends more northward.

\subsection{GDS results}

After analysing MT data, additional quantitative information complementary to MT can be obtained from the same data by switching to the GDS approach (section 2.4) where only the three orthogonal magnetic components are used in the absence of electric fields. This evaluation of the regional differences of the variation fields of different sites (geomagnetic depth sounding) comes at no additional cost. That is to say, the results presented here under GDS are obtained from the MT data with no additional hardware or field procedures. The only requirement for GDS evaluation is a permanent running site during the campaign that serves as a reference site. In our case, since our data was measured in 3 separate years, we initially used Alice Spring magnetic observatory as our reference site. The data format from Alice spring is not the same as our data acquired using data loggers developed at the University of Göttingen by Steveling and Leven (1992). Our format is the goe which is a binary (direct access) format. The record length is 256 bytes. The first 4 records (1024 bytes) contain header information including run time, site-name, sampling rate and number of records in the file. The next records starting from the 5th contain all data for the first saved component followed by the data of the second component (starting with a new record) and so on. Detail info can be found in the RAP data-logger manual (Steveling, 2000). Therefore, a program was written (appendix D.3.1) to convert the one-second time resolution vector data from Alice Spring to the .goe format before proceeding to evaluate the GDS data. 
From the MT transfer functions, the site ARG is closer to a 1D conductivity structure than Alice Spring and given its good data quality, it is more suitable to be used as a reference site. We therefore used the program gdsswap as described in section 2.4 to change the reference site from Alice Spring to site ARG. The reference site ARG or Alice spring is much remote from sites to the extreme eastern part of the array and therefore the perturbation tensors at long periods should be interpreted with care because of Sq effects (section 2.1). The perturbation tensors at shorter periods were found distorted; we therefore avoid the interpretation of the perturbation tensors at periods below 100 s. Figures 4.13 and 4.14 show contour plots of the real parts of horizontal and vertical perturbation tensor elements from our GDS analysis. 

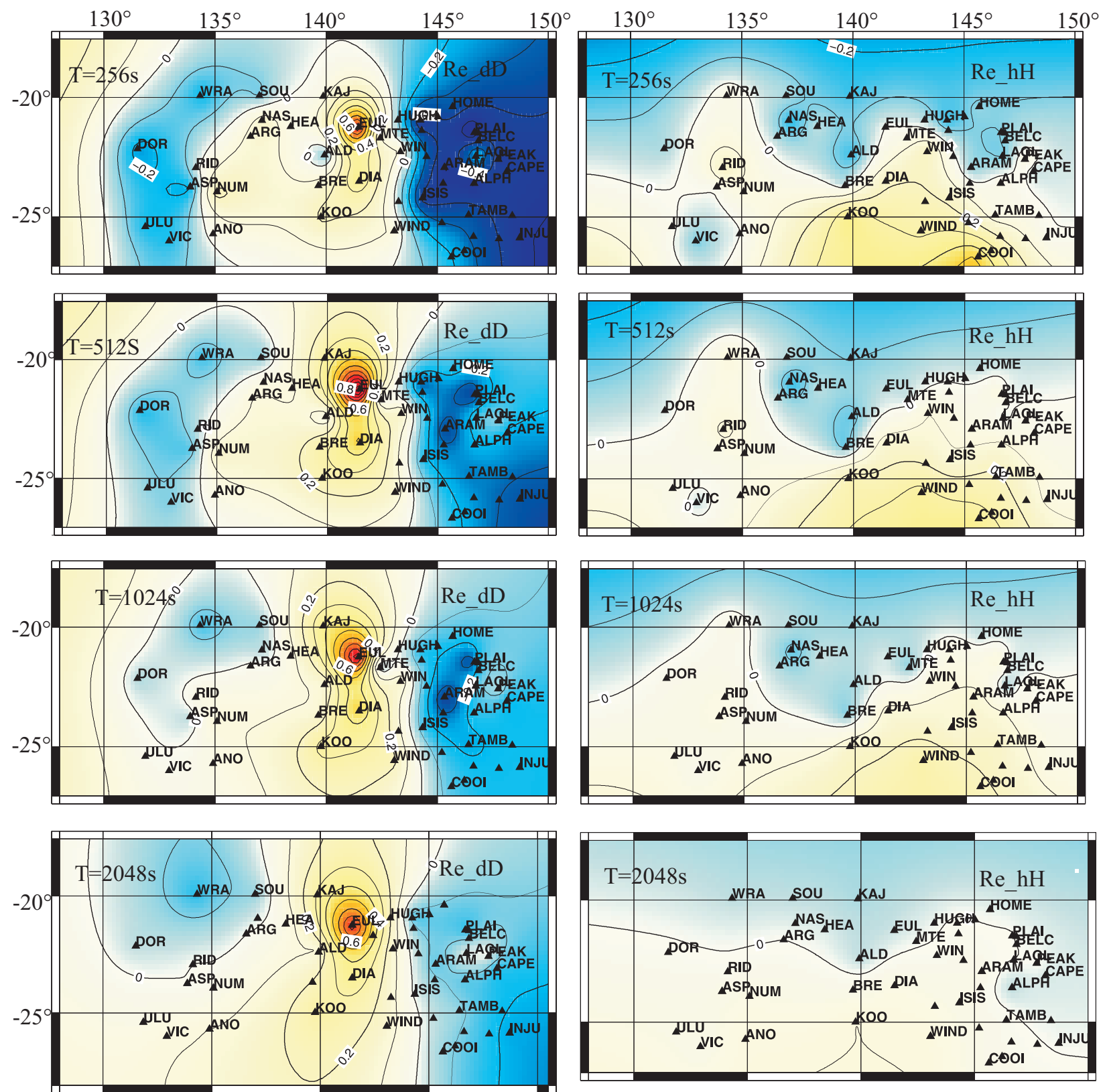

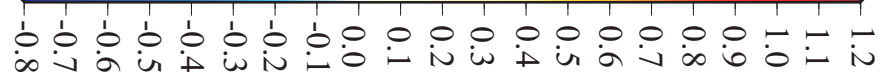

Figure 4.13: Real parts of the horizontal perturbation tensor $\left(d_{D}\right.$ and $\left.h_{H}\right)$ with site ARG as reference site for four different periods. 

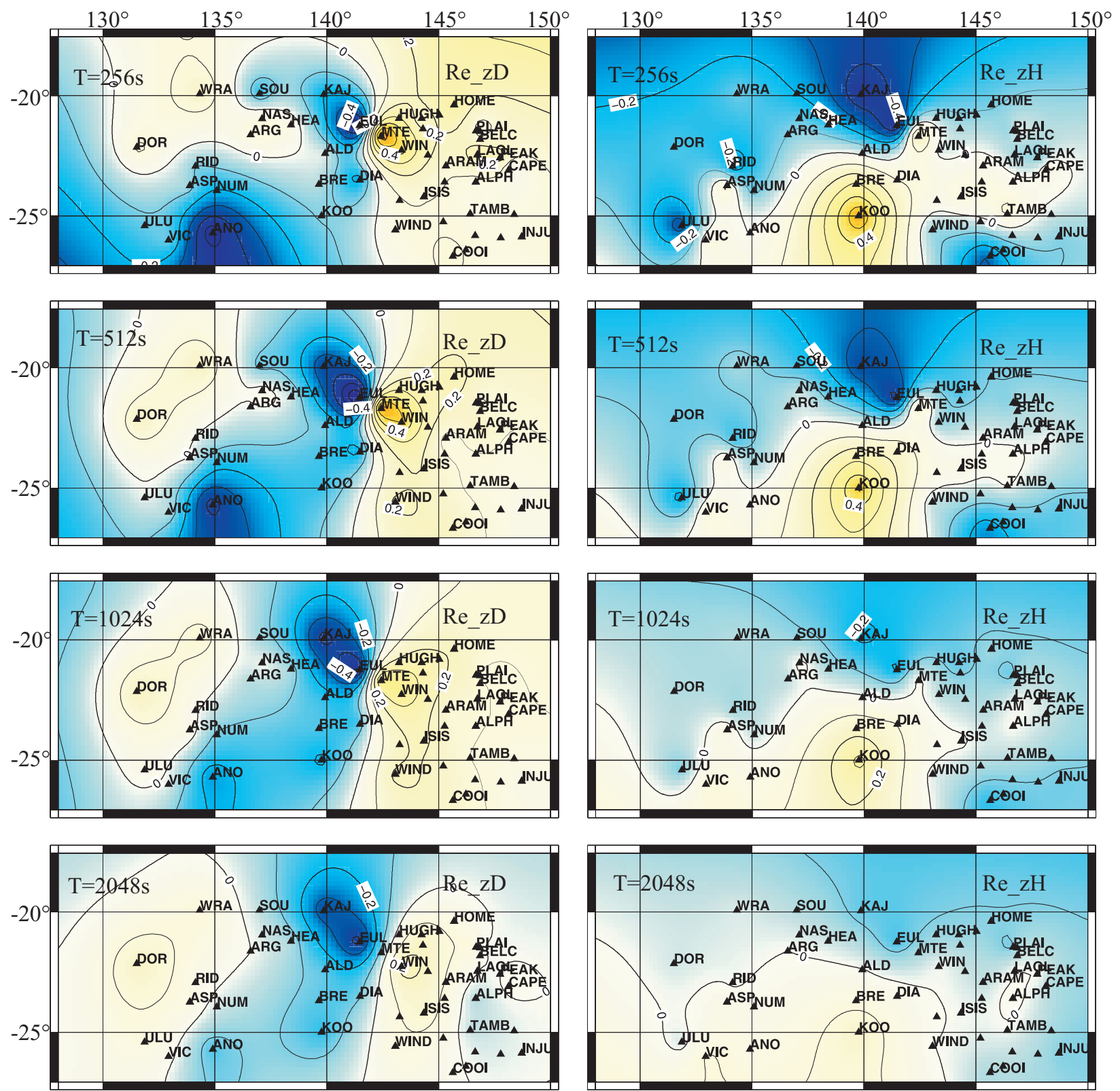

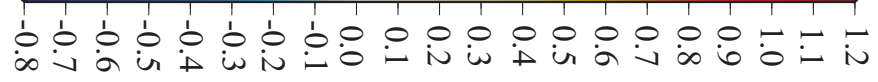

Figure 4.14: Real parts of the vertical perturbation tensor $\left(z_{D}\right.$ and $\left.z_{H}\right)$ with site $\mathrm{ARG}$ as reference site for four different periods.

Figures 4.13 and 4.14 show the contour plots of the $d_{D}, h_{H}, z_{D}$ and $z_{H}$ components of the perturbation tensor for periods $256 \mathrm{~s}, 512 \mathrm{~s}, 1024 \mathrm{~s}$ and $2048 \mathrm{~s}$ in an unrotated coordinate system. Assuming that the conductivity of the subsurface at the reference site, $\mathrm{ARG}$, is $1 \mathrm{D}$, the increased $d_{D}$ amplitudes observed between longitude $140^{\circ} \mathrm{E}$ and $142.5^{\circ} \mathrm{E}$ indicate increased magnetic fields in the east-west direction and therefore increased current densities in the north-south direction and thus a north-south striking conductor along 
these longitudes. This is in good agreement with the induction vectors discussed above. Increased $d_{D}$ amplitudes (figure 4.13) are observed in regions $2 \& 3$ (figure 4.4) with maximum $d_{D}$ amplitude at the site EUL. This means that the north-south striking anomaly is centred on site EUL; the maximum $d_{D}$ amplitude is at the centre of a north-south conductive block (section 2.4). As earlier mentioned, region 3 extends to KOO and the increased $d_{D}$ extends to KOO meaning that the anomaly extends to this point. Does this contradict our earlier conclusion that the induction vectors at $\mathrm{KOO}$ are influenced by a different conductor? To answer this question, it is important to look at the $h_{H}$ perturbation tensors. By examining the $h_{H}$ perturbation tensor (figure 4.13), one observes that its amplitude has a maximum along the line joining $\mathrm{KOO}$ and $\mathrm{COO}$, that is approximately east-west meaning the existence of an east-west conductor at this position. In addition, at KOO the $d_{D}$ amplitude is less than that of $h_{H}$; therefore the effect of the east-west conductor at KOO dominates that of the north-south conductor extending from EUL. This is further evident by the induction vector at KOO which is pointing approximately south-north and therefore perpendicular to the east-west conductor. Since both the $d_{D}$ and $h_{H}$ perturbation tensors at KOO have increased amplitudes, they are characterised by two conductors, a north-south and an east-west striking conductor. By inspecting the vertical perturbation tensors (figure 4.14), especially $z_{D}$, we see that to the west of regions 2 and $3, z_{D}$ is negative and positive to the east which is in agreement with our conclusion from section 2.4 that $z_{D}$ has maximum amplitude at the borders of a conductor. From the GDS transfer functions, one can see clearly a band like conductor running east of KAJ through EUL to KOO. This path is in the vicinity of the "Tasman Lines" discussed in section 3.6. We will come to a conclusion on the issue of a Tasman Line after we summarise evidence from inductions arrows, strike direction and from the results of modelling. We now proceed to look at GDS strike directions.

\subsubsection{GDS strike directions}




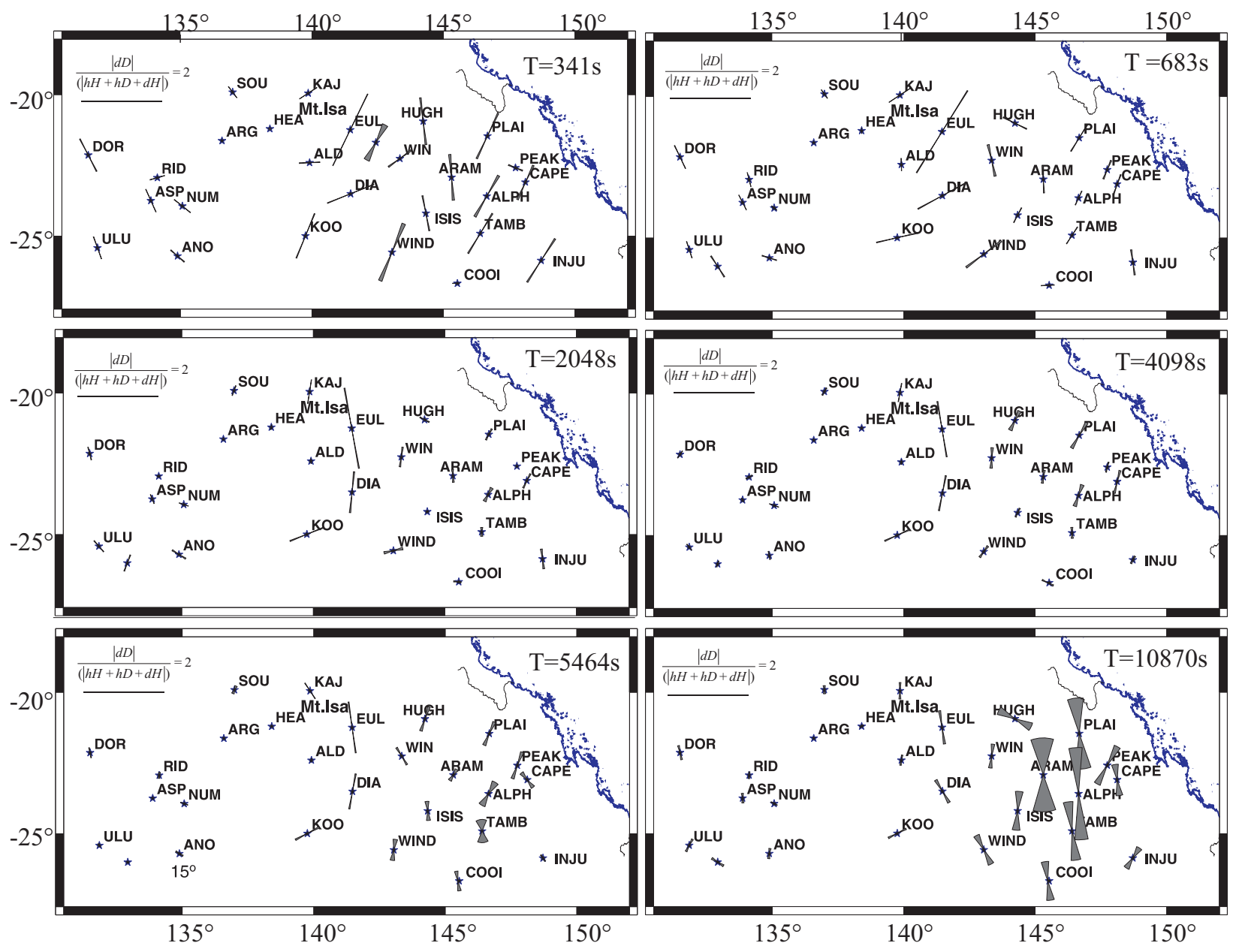

Figure 4.15: GDS strike angles for six different periods indicated on the diagrams. Strike angles were obtained by maximizing the $d_{D}$ perturbation tensor element with respect to the other horizontal perturbation tensor elements as given by the equation in the diagram. The equation was discussed in section 2.4 where it was represented by $A_{s}$ and is an amplitude indicating the strike strength in the north-south direction. The shapes in the east at $\mathrm{T}=10870 \mathrm{~s}$ represent the error bars of the strike calculated 
Figure 4.15 shows GDS strikes angles (calculated using equation (2.81) in section 2.4) for different periods shown in the figure. The strike amplitude $A_{s}$ is obtained by maximizing the $d_{D}$ perturbation tensor element with respect to the other horizontal perturbation tensor elements as given by the expression for $A_{s}$. Unlike the MT strike directions in figure 4.11 that show a spatially consistent pattern but do not concur well with the induction arrows' direction in figure 4.9, the GDS strike angles are consistent and also in accordance with the induction arrow directions. That is, there is a north-south striking direction especially along KAJ, EUL and DIA (regions 2 and 3 ) which is perpendicular to the directions of induction vectors. The difference between the MT strike directions and the GDS strike directions may be explained in terms of the difference in the nature of magnetic and magnetotellurics transfer functions. Along region 3, we depict a band like conductor, the Carpentaria conductivity anomaly running north-south. While the magnetic fields that map mostly regional structures are most sensitive to this band like regional conductor, the electric fields in addition to the conductor also react to small scale deviations from the straight band. The MT transfer functions which are a function of the electric fields will therefore delineate 3D structures while the GDS transfer functions map the predominant conductive band. Note that the induction vectors like the GDS perturbation tensors are independent of the electric fields-hence the agreement. Therefore the MT transfer functions provide a detailed resolution of the local structures while the GDS results delineate the dominant regional structures.

To resolve detail structures using $\mathrm{MT}$, the site spacing must be reasonably small. In our study, this is not the case since we are interested in long periods investigation of regional structures that characterise the Tasman Line. However, it is also important to note that although the MT strike directions do not concur with that of the induction vectors they do not look locally distorted. They seems to be sptially consistent and may render our explanation for the difference in terms of small scale structures weak. Because of this ambiguity which cannot be satisfactory resolved, to be on the save side, we will concentrate on the interpretation of the GDS strike angles that concur with the induction vector directions and are cemented by the fact that, in general magnetic transfer functions provide good measures for regional scale structures.

If one examines figure 4.15 closely, at short periods (341 and $683 \mathrm{~s}$ ) the direction is essentially north-south, however, tilted northeast-southwest to the east and tilted northwestsoutheast to the west. This can be associated to current channelling described in section 2.5. This is the period range within which the effect of the Carpentaria anomaly is very strong (period range of maxmum induction)-hence a strong channelling effect at these periods (section 2.5). At longer periods, especially starting at $2048 \mathrm{~s}$, regional information from the strike angles becomes very interesting and visible indicating a clearly north-south strike direction from KAJ through EUL to DIA (regions $2 \& 3$ ). At KOO, the strike is tilted, we will come to this shortly. There is virtually no strike both to west and to the east of this region. The area appears to be divided into two along KAJ to KOO judging from the magnitude of $A_{s}$, a measure of the strike's strength. The distinct nature of the strike direction in the region is most likely to be attributed to a geological boundary that is running from north to south. At a period of $10870 \mathrm{~s}$, the strike directions with large error bars to the east do not carry any geological information; there are due to Sq effects (section 2.1). We came to this conclusion after looking at the behaviour of the $h_{H}$ and $d_{D}$ perturbation tensor elements at periods from $9000 \mathrm{~s}$ and above for the sites with this 
feature. The $h_{H}$ and $d_{D}$ perturbation tensor elements were expected to converge to zero at long periods. Nonetheless at periods above $9000 \mathrm{~s}$, they suddenly diverged from zero with $h_{H}$ becoming more positve and $d_{D}$ more negative given an impression of an anomaly in the mantle. A careful examination of the perturbation tensor favoured Sq effects that are prominent at long periods.

We now attempt to explain why the strike direction at KOO is not perfectly north-south as expected so as to make the boundary undisputed. It was already shown above that there is a conductor joining $\mathrm{KOO}$ and $\mathrm{COOL}$ which is running east-west (figure 4.10) judging from $h_{H}$ perturbation tensor at these sites and from the induction vector at KOO. We also concluded that apart of the north-south conductor, there was another conductor to the south influencing its induction arrow. Therefore it is clear that the current flowing from the north along what is becoming clear as the Tasman Line, divides around KOO; some continues south while part of the current runs west-east along KOO and COOL, hence the direction obtained at KOO.

Judging from the induction vectors, the GDS perturbation tensor elements and the GDS strike directions, we have delineated a band like conductor running east of KAJ through EUL to KOO, which seems to divide Australia into two. The delineated path (band like conductor) shows a distinction even at greater depths and falls in the vicinity of the much debated Tasman Line. A detailed model still to be presented in the next chapter will shed more light on the nature of the Tasman Line which at this point still looks broad. 


\section{3D modelling of the data}

This chapter focuses on the regional 3D modelling developed to identify the major conductivity structures of the study area (figure 3.2). We interpreted the characteristics in this dataset by forward modelling performed with the modified version (Mackie and Booker, 1999) of the 3D algorithm developed by Mackie et al. (1994). The original algorithm by Mackie et al. (1994) uses an integral form of Maxwell's equation to derive a finite difference approximation for the magnetic fields. The algorithm makes use of a model with input resistivities to compute the electric and magnetic fields in each cell of the model. The field values at the surface of the model are then used to estimate the MT and GDS transfer functions at each surface cell of the model. The computed transfer functions are then compared with measured data. Essentially, the forward modelling code is an iterative procedure involving progressive trial-and-error fitting of data by (i) computing the responses of an input model, (ii) comparing these responses with measured data, (iii) modifying the model where the data do not fit adequately and then (iv) re-computing the responses, until a suitable fit to the measured data is achieved.

The major difference between the original algorithm and the modified one used in this thesis concerns the boundary conditions and the method for initializing the fields. The original program computed 2D fields along each slice through the 3D model. These fields were then used to set the boundary values and to initialize the interior of the model. The $2 \mathrm{D}$ fields were computed by a separate program and then passed to the $3 \mathrm{D}$ program via a disk file. The new version no longer requires a separate computation of the boundary fields. It assumes that $2 \mathrm{D}$ structures parallel to each edge continues uniformly to infinity in the direction normal to the edge. The edges parallel to the source magnetic field will have electric current flowing normal to them and so TE mode calculations are carried out. The edges perpendicular to the source magnetic field will have current flowing parallel to them giving rise to zero tangential magnetic fields. More details on the source code and how to use the program, especially the numerous gridding rules can be found in the documentation of the program (Mackie \& Booker, 1999).

Although a 3D forward modelling follows a trial and error fitting approach, certain procedures must be followed in order to start off with a reasonably good initial model. In this regard, in order to develop a good 3D forward model, a stepwise approach is imperative. First, one must define the major structures of the model. This is possible from either a direct investigation of the electromagnetic transfer functions (see our classification of the MT transfer functions into five different regions in section 4.3) or from a detailed analysis of the measured data as in chapter 4 (induction vectors, skew values, strike direction and plots of GDS perturbation tensors). These analyses give already an idea of structural boundaries and regional structures to be included in the model.

Secondly, the significance of the response of the major structures in the model is checked by successive variation of their parameters such as resistivity, vertical and lateral extent. 
Then one can proceed to refine the model by a trial and error fitting.

\subsection{D modelling of the Australian data}

The major regional structures of the study area are deduced from the MT and GDS transfer functions (chapter 4) and from knowledge about geological and geophysical information of the area from previous studies. According to the review of the study area, the most prominent features that must be considered in developing an initial model include (i) the highly conductivity sedimentary Eromanga basin in the southeast (the MT transfer functions have a small phase of about $15^{\circ}$ at short periods- a top thin conductive layer is included in the part of the model representing this region) (ii) the resistive Mt. Isa block, (iii) the Carpentaria conductivity anomaly at the eastern boundary of the Mt. Isa block as clearly revealed by the GDS perturbation tensors, (iv) an approximately 1D crustal structures (ARG was chosen as a reference site for GDS) in the western part of the array and (v) a moderately resistive environment east of the Carpentaria anomaly extending to the Coral Sea. The level to which these structures extend can be approximated from the period dependence of the transfer functions and parameters such as skew values and inductions arrows. For example, from the $d_{D}$ perturbation tensor, the conductivity anomaly (Carpentaria conductivity anomaly) at site EUL and its environ extends to a period of at least $4000 \mathrm{~s}$. Large skew values at site KAJ at longer periods indicate that $3 \mathrm{D}$ structures at this site judging from the large phase split originate at longer periods while large skew values at site HUGH at short period suggest that 3D structures at the site are in the upper crust. The region running north-south from east of KAJ through EUL to KOO (our postulated Tasman Line), induction vectors (figure 4.9) unlike in the entire surveyed region remain strong in strength up to a period of at least 4000 s. Further more, essential information to consider in the initial model from the period dependence of transfer functions include :(i) the limited period range of the small phase of transfer functions in the sedimentary Eromangan basin-the sediments are highly conductive, about $1 \mathrm{~S} / \mathrm{m}$ and extend to about $2 \mathrm{~km}$, (ii) the Mt. Isa block is highly resistive at shallow depths (apparent resistivities at site ALD (appendix B, figure B.2) are at least $1000 \Omega m$ at the shortest periods and fall to very small values, less than $100 \Omega m$ at long periods. This, in addition to large phases at longer periods means that conductivity increases with depth in this region. (iii) the conductivity of the Coral Sea to the east is set at $0.5 \mathrm{~S} / \mathrm{m}$ up to a depth of about 2000 $\mathrm{m}$.

In order to start with a reasonably good model representative of the major geological structures in the region of interest, additional knowledge from other methods (chapter 3 ) has been used to refine the location and extent of these geological structures. These include: (i) knowledge on the extent and depth of the great Artesian Basin (figure 3.3) with a thickness varying between at least $100 \mathrm{~m}$ on the Basin extremities and over 3,000 $\mathrm{m}$ in the deeper parts of the Basin (GABCC, 1998) (ii) information about the Carpentaria conductivity anomaly from previous electromagnetic induction studies (Chamalaun et al., 1999; Lilley et al., 2003; Hanekop, 2006) (iii) Crustal/lithospheric thickness variations of the Australian continent (section 3.1) (iv) Knowledge from gravity and magnetic anomalies study of the area (chapter 3$)$ 
The above observations were taken into consideration during the development of the initial model. Our study area is somehow large, about $670 \mathrm{~km}$ by $1660 \mathrm{~km}$. A fine gridding that will resolve local structures will lead to a model that is too slow to calculate and one will have to wait for at least 12 hours before making a modification. Since our interest lies in the resolution of regional structures (geological boundaries) with emphasises on the Tasman Line, in the central region of our study area where the data density is greatest, the finite difference grid spacing in the horizontal direction is $10 \mathrm{~km}$ by $20 \mathrm{~km}$. The spacing increases outwards from the central region following the gridding rules. This grid spacing is quite reasonable and sufficient to resolve regional structures given that our site spacing is about $200 \mathrm{~km}$ with neighbouring sites having similar transfer functions, an indication that regional structures were resolved by our site spacing (section 4.3). In the vertical direction, the depth of the grid nodes also increases according the gridding rules. The mesh spacing in the uppermost $3400 \mathrm{~m}$ (4 nodes) of the model was chosen to represent the small phase of sites in the Eromanga basin and the near-surface resistivity structure at each site. In all, the finite difference grid used $51 \times 51 \times 34$ nodes in the $\mathrm{x}, \mathrm{y}$ and $\mathrm{z}$ directions, respectively leading to a total model volume of about $1460 \mathrm{~km} \times 2860 \mathrm{~km} \times 860$ $\mathrm{km}$.

The difficulty with forward modelling with a large number of sites is to find an efficient way of comparing the observed and model responses. The approach followed here was, first, to fit maps of GDS perturbation tensor elements at different periods with corresponding maps of the calculated GDS perturbation elements and second, to fit the phases of the MT transfer functions and also compare induction vectors. This approach ensures that the 3D nature of the data set is honoured, and it also avoids distortion produced by near-surface heterogeneities (geological noise)-hence the effects of static shift are avoided. Notwithstanding, the phase response only contains information on changes or gradients in the resistivity and does not allow the length scale of the resistivity structure to be determined. This is not a serious problem for the investigation of regional structures.

To achieve the first task of fitting the GDS perturbation tensors, since our reference site (ARG) is not exactly $1 \mathrm{D}$ as required by the GDS method, we first tried to reproduce the MT transfer function at site ARG (figure 5.1) before proceeding with our GDS modelling. 


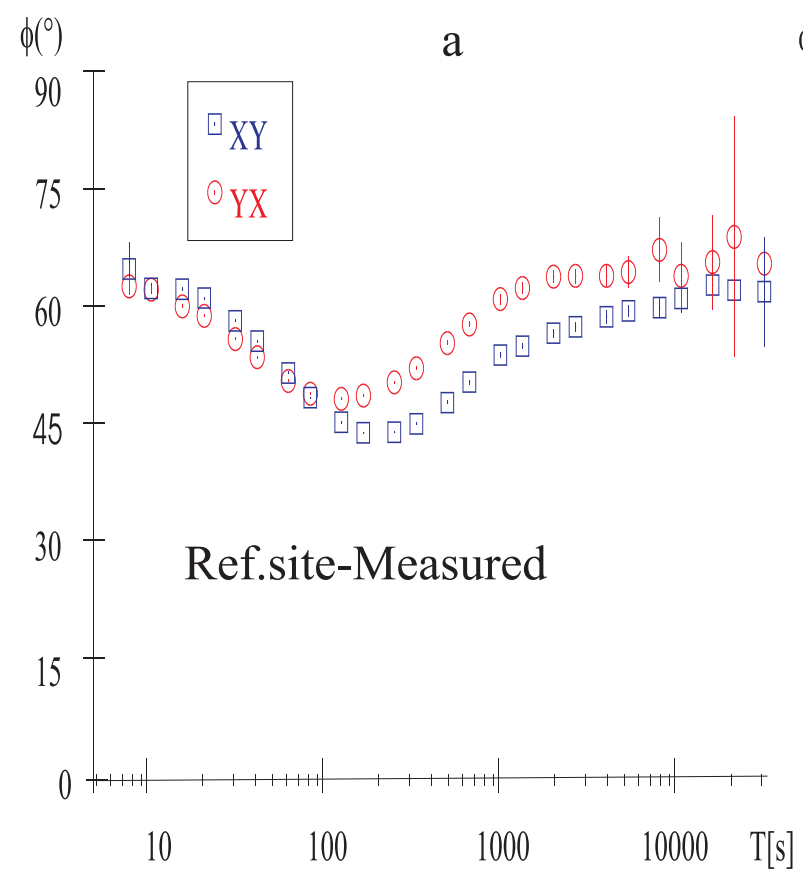

$\phi\left({ }^{\circ}\right)$

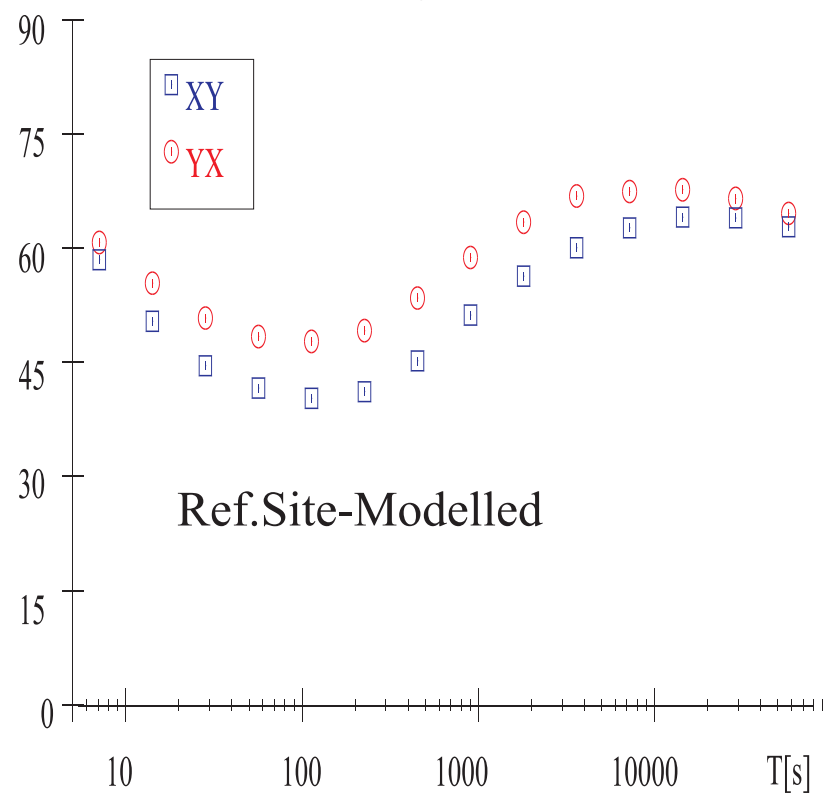

Figure 5.1: (a)Measured MT transfer function at the reference site, ARG and (b) calculated MT transfer function at the reference site(phase only).

It is important to note that the result shown in figure $5.1 \mathrm{~b}$ is obtained from the final 3D forward model. This result means that even if conductivity gradients are present at the reference site and or in its vicinity, it is already taken into account in the process of reproducing the MT transfer function at the reference site. In this case anomalous fields cannot be interpreted as normal ones unlike the case where anomalous fields will be interpreted as normal ones if the reference site is not 1D.

To proceed with the fitting of the perturbation tensor elements, we began with the horizontal ones. We observed, that sites in the entire array between longitudes $139^{\circ} \mathrm{E}$ and $143.5^{\circ} \mathrm{E}$ have positive real $d_{D}$ perturbation tensor elements and magnitude of at least 0.4 for a period range between $256 \mathrm{~s}$ and $1024 \mathrm{~s}$ with site EUL having an extremely large amplitude of about 1.2 between $500 \mathrm{~s}$ and $700 \mathrm{~s}$, see figure 4.13 . The positive $d_{D}$ perturbation tensor elements between longitudes $139^{\circ} \mathrm{E}$ and $143.5^{\circ} \mathrm{E}$ indicate strong currents flowing north-south along these longitudes. It was concluded from section 2.4 that currents flowing north-south leads to the $d_{D}$ anomaly. These were represented with resistivities between $0.5 \Omega m$ and $10 \Omega m$ up to a depth of $3400 \mathrm{~m}$, except sites KAJ and ALD that belong to Mt.Isa and therefore have a resistive crust. That is the crust at site KAJ is very resistive and is represented with a resistivity of at least $3000 \Omega \mathrm{m}$. Site ALD, although falls in the sedimentary basin has the same geology as that of Mt.Isa. Because it is part of the sedimentary basin, it has a thin top conductive layer and because its geology is that of Mt.Isa, the thin conductive layer is followed by a very resistive crust with its resistivity decreasing with depth. This property at this site was discovered when an attempt was made to fit the MT phase. To summarise, resistivities at sites ALD and KAJ which both belong to the Mt.Isa block and to the $d_{D}$ anomaly between longitudes $139^{\circ} \mathrm{E}$ and $143.5^{\circ} \mathrm{E}$ were found to decrease with depth. This is in agreement with our conclusion from sec- 
tion 4.3.2 that small skew values at site KAJ at short periods indicate that 3D structures originate at long periods that is the conductivity structure within site KAJ is $1 \mathrm{D}$ at short periods. These features were adequately represented in figure 5.2.

At greater depths, the resistivites along the north-south conductor described above were fixed as in the final model to obtain the positive $d_{D}$ perturbation tensor elements between longitudes $139^{\circ}$ and $143.5^{\circ} \mathrm{E}$. As can be seen from the final model (figure 5.2), the conductor that produced the $d_{D}$ anomaly especially at site EUL with the greatest amplitude is limited to the crust.

\section{Fitting the other horizontal perturbation tensor element $-h_{H}$}

Again from figure 4.13, we observe that the $h_{H}$ perturbation tensor element is strongest between sites COOL, latitude $-26^{\circ} \mathrm{S}$ and $\mathrm{KOO}$, latitude $-25^{\circ} \mathrm{S}$. This indicates east-west currents since they lead to $h_{H}$ anomaly (section 2.4). These were represented accordingly in the model and varied until the $h_{H}$ perturbation tensor elements' amplitude was obtained. The conductance can be again deduced from the final model (figure 5.2).

\section{The Vertical perturbation tensor elements}

Looking at the vertical transfer function, $z_{D}$ in figure 4.14, there is an interesting feature around longitude $142.5^{\circ} \mathrm{E}$ for all latitudes in the array. Observe that sites to the west of longitude $142.5^{\circ} \mathrm{E}$ have a strong negative $z_{D}$ value while those to the east of longitude $142.5^{\circ} \mathrm{E}$ have a positive value. This implies that there is a conductor centred on longitude $142.5^{\circ} \mathrm{E}$ which may be slightly different from the one that we described above for the $d_{D}$ anomaly. We will explain shortly why we think it is different. The negative value of $z_{D}$ west of longitude $142.5^{\circ} \mathrm{E}$ and the positive value to the east indicate the boundaries of this conductor, again see section 2.4 where we showed that $z_{D}$ has maximum amplitude at the boundary of a north-south striking conducting anomaly. We therefore fitted accordingly this anomaly at longitude $142.5^{\circ} \mathrm{E}$ in the model. In an attempt to fit the magnitude of calculated perturbation tensor element, $z_{D}$ to the measured, it was discovered that this can only be accurately represented at long periods if we extend this conductor much deeper. A good fit was obtained when the conductor was extended to at least $80 \mathrm{~km}$ deep, which is already in the mantle. The conductor that defined the $d_{D}$ anomaly was limited to the crust and much broader, between longitudes $139^{\circ} \mathrm{E}$ and $143.5^{\circ} \mathrm{E}$. The conductor that defines the $z_{D}$ anomaly is thin and extends to the mantle, it is common to the $d_{D}$ anomaly only in the crust and therefore is slightly different. These anomalies have been represented accordingly in the 3D forward model. One can't see a distinction in the crust between the two anomalies, but the distinction is clearly visible with increasing depth. We will elaborate and broaden on the implication of this later.

The other vertical perturbation tensor, $z_{H}$ was represented in the model by including an east-west conductor south of site KOO. The final model is ploted in figure 5.2 and a vertical cross section of this model about latitude $21.430^{\circ} \mathrm{S}$ is given in figure 5.3 . 


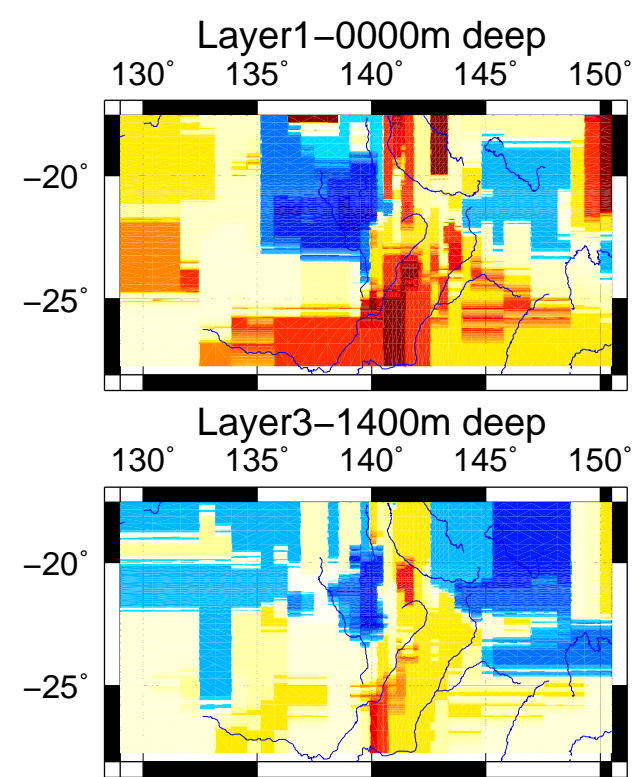

Layer4-2400m deep

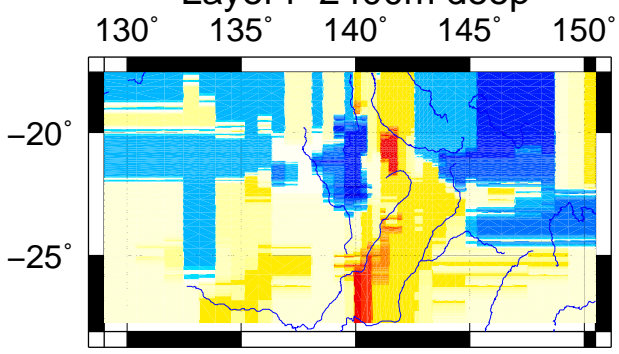

Layer5-3600m deep
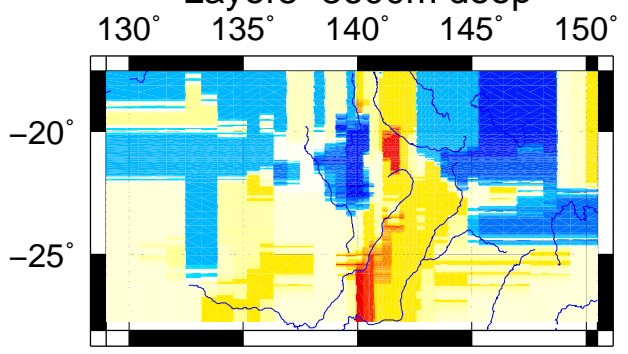

Layer9-11800m deep

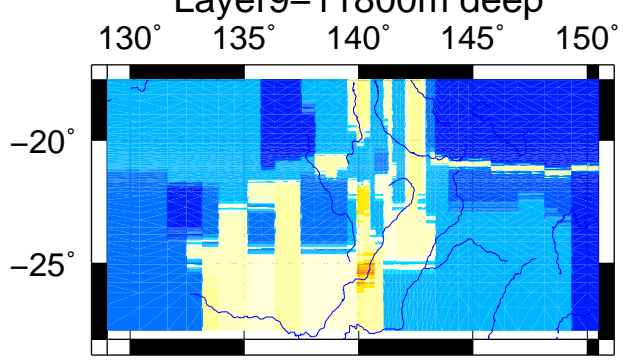

Layer13-28700m deep

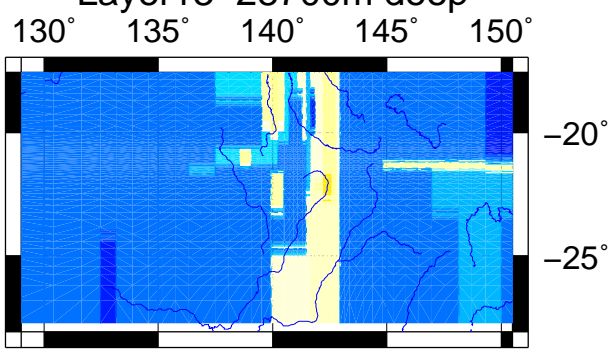

Layer16-43100m deep
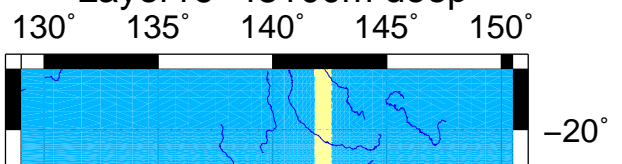

Layer19-65600m deep

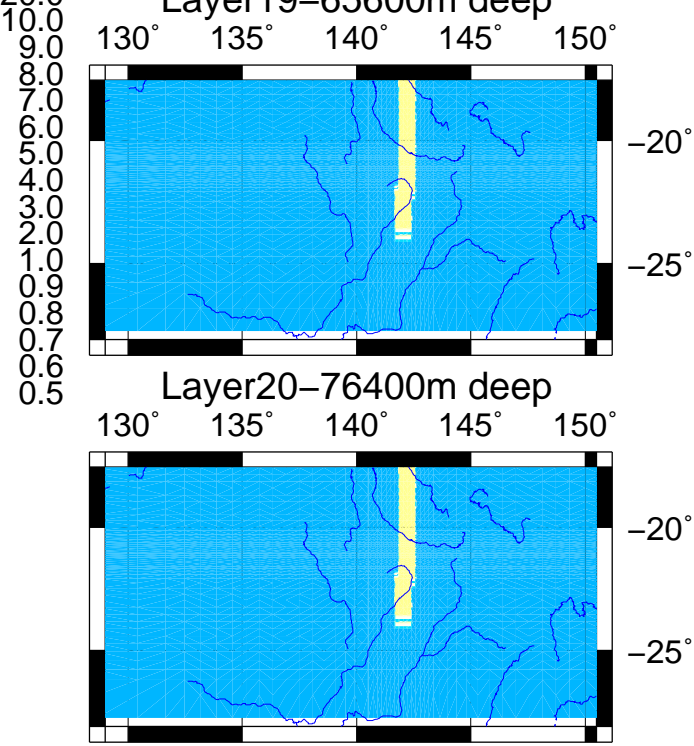

Layer21-89900m deep

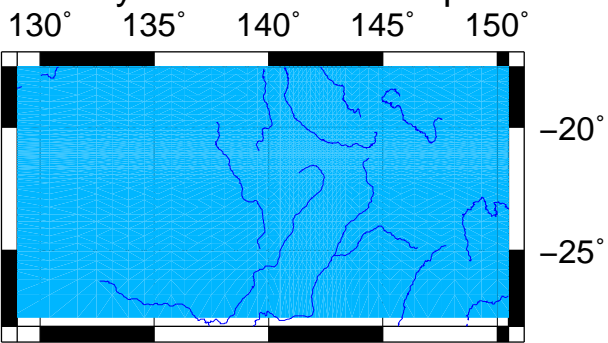

Figure 5.2: Resistivities of some layers in the final 3D forward model (model1) of this project's dataset. The layer number and depth (in $\mathrm{m}$ ) of the top of each layer plotted is labelled in $\mathrm{m}$ in the figure. The resistivities between two layers equals the resistivity of the upper layer. 


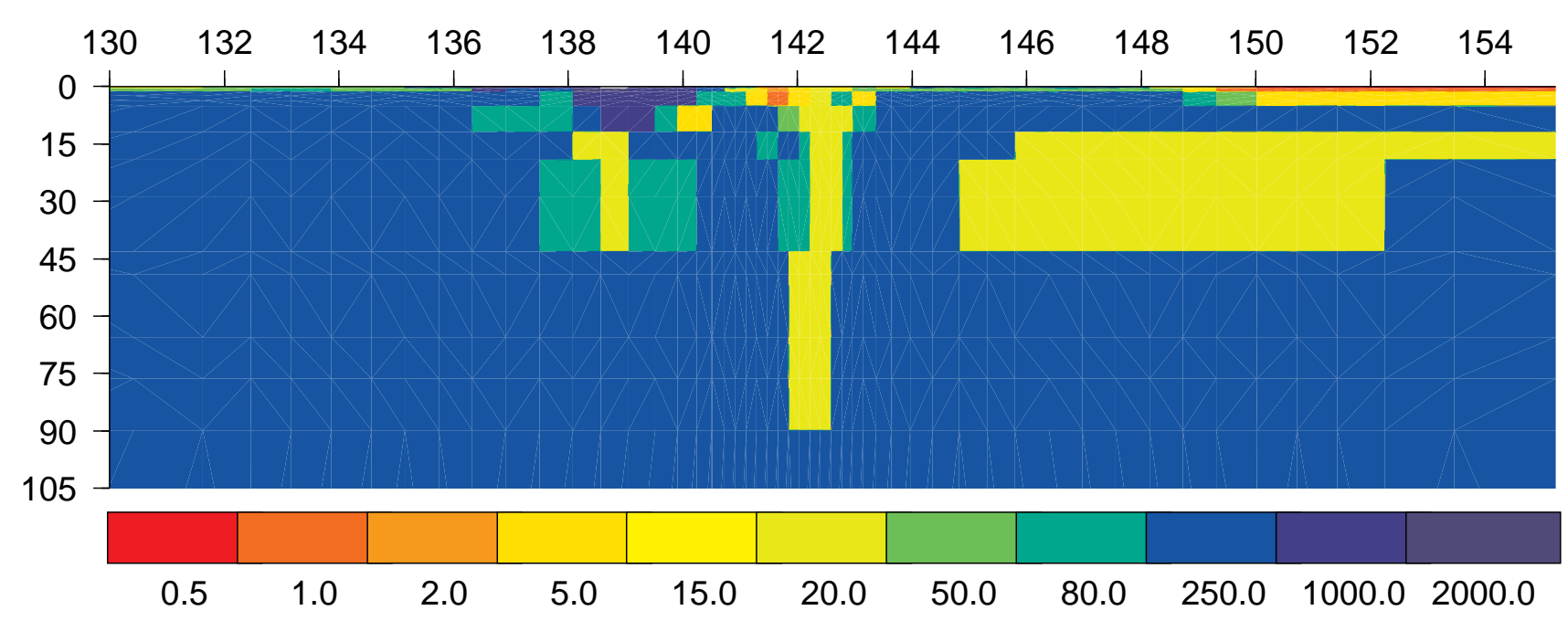

Figure 5.3: Vertical cross section of the final model about latitude $21.430^{\circ} \mathrm{S}$. The vertical axis gives the depth in $\mathrm{km}$ and the horizontal axis represent the longitudes in degrees. The scale is in Ohm.m

The conductor shown in the vertical cross section from longitude $150^{\circ} \mathrm{E}$ and above represents the Coral Sea while the conductor at $142.5^{\circ} \mathrm{E}$ and extending to a depth of $90 \mathrm{~km}$ is our postulated Tasman Line.

\subsection{Results from the model compared with measured data}

\subsubsection{GDS results}

Figures 5.4 and 5.5 below show the results of our modelled horizontal transfer functions compared with the measured ones. 

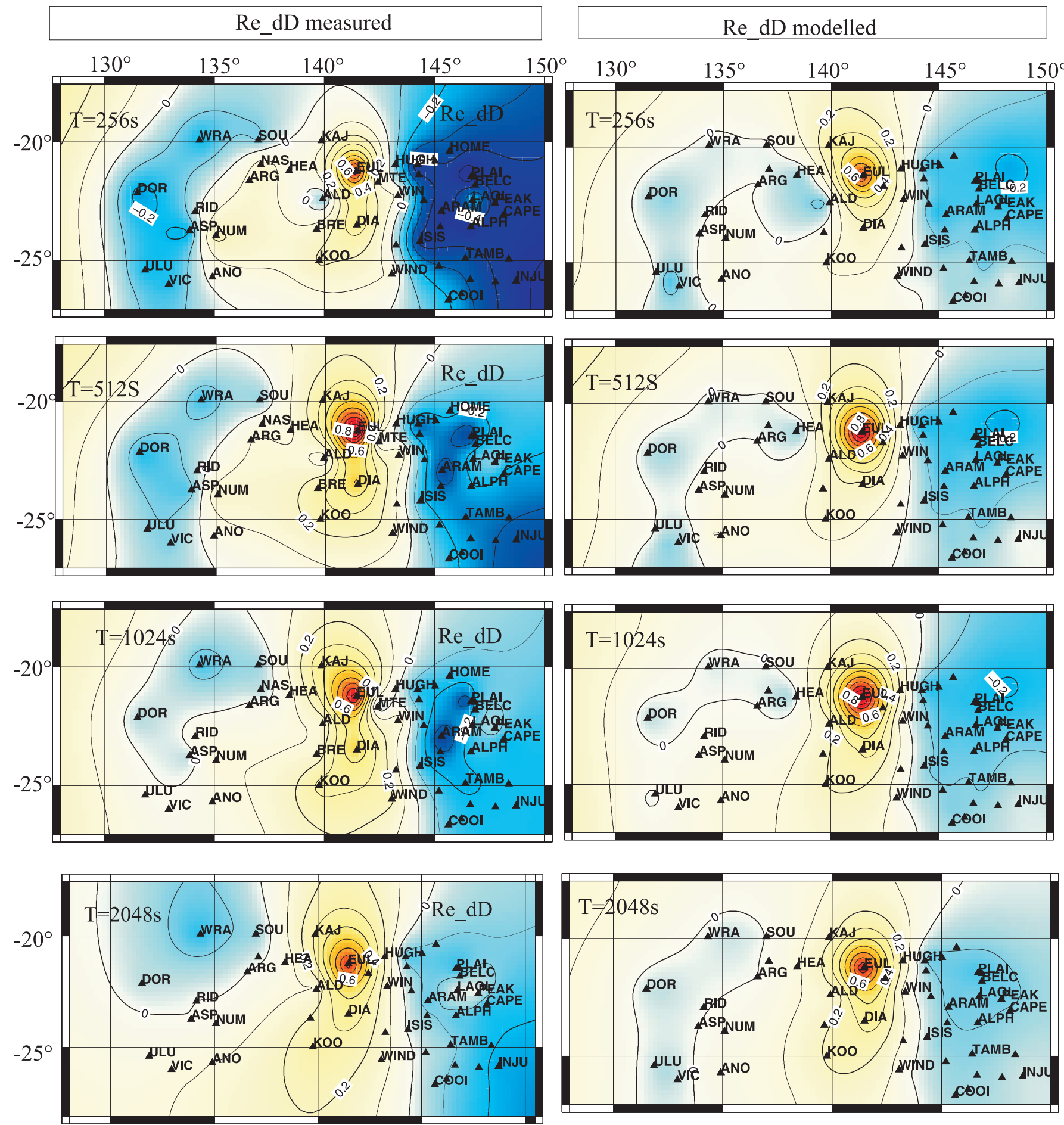

$$
\text { bo }
$$

Figure 5.4: Measured and modelled real parts of the $d_{D}$ perturbation tensor elements at four different periods compared. Sites used for the interpolation are labelled on the map. 
The model has produced the contour lines of the measured $d_{D}$ perturbation tensor. Given the difficulty involve in 3D forward modelling by trial fitting, the modelled $R e \_d_{D}$ perturbation tensor elements are very satisfatory when compared to measured ones.

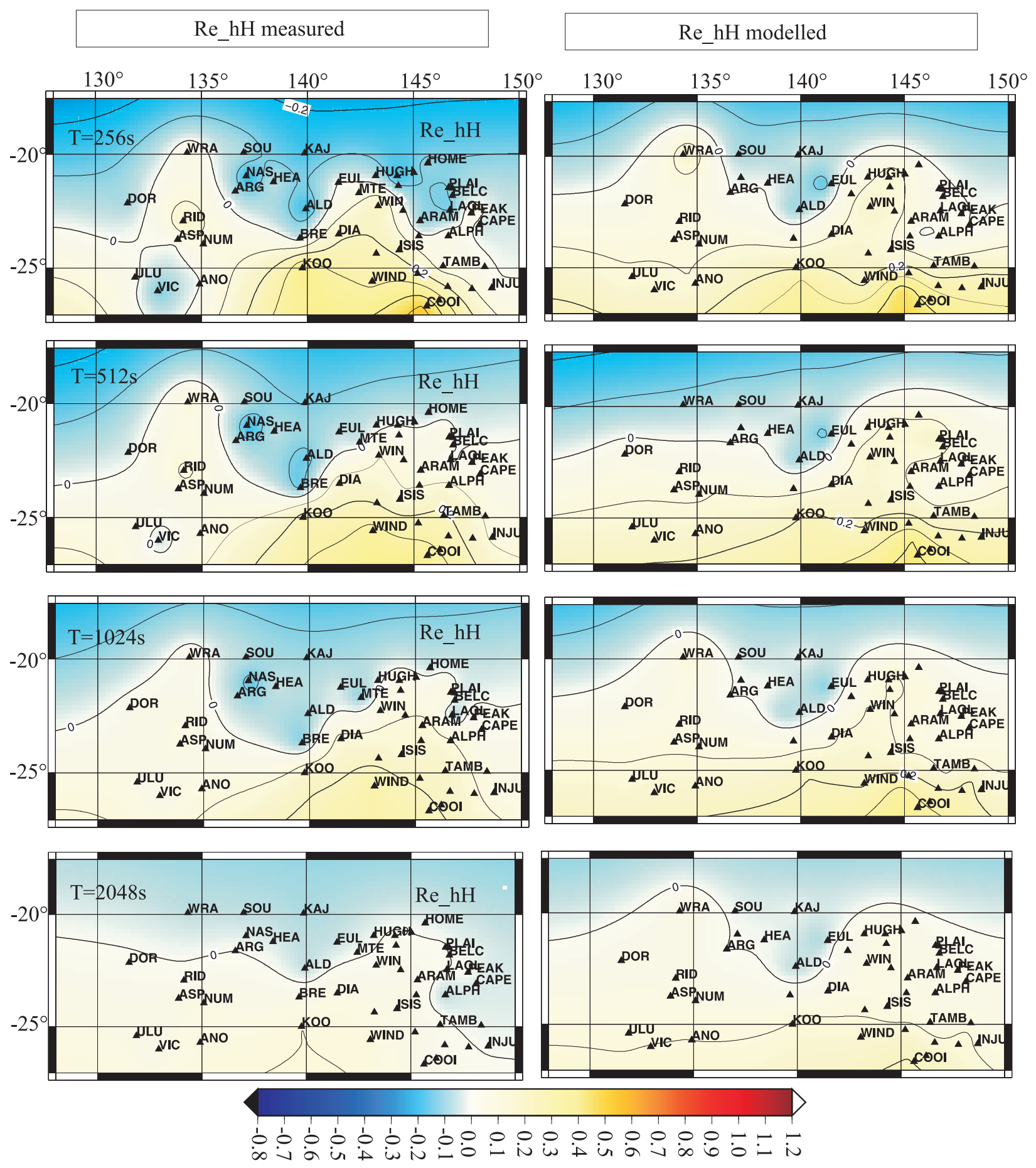

Figure 5.5: Measured and modelled real parts of the $h_{H}$ perturbation tensor elements at four different periods compared. Sites used for the interpolation are labelled on the map. 
The modelled real part of the $h_{H}$ perturbation tensor elements have also reproduced the measured one with at least an $80 \%$ success as can be seen in the figure 5.5 above. The west-east striking conductor included in our model around latitude $25^{\circ}$ south accounts for the fit of $R e \_h_{H}$ perturbation tensor elements especially at sites KOO and COOL. This conductor equally helped to reproduce perfectly the induction arrow at site KOO as will be seen in the next section; MT results. Figures 5.6 and 5.7 are the output for the modelled vertical perturbation tensor elements compared with the measured ones. The match between the measured and the modelled ones are suprisingly very good with a percentage success also above $80 \%$ especially for the $z_{D}$ perturbation tensor element which is very important for the mapping of a boundary. 


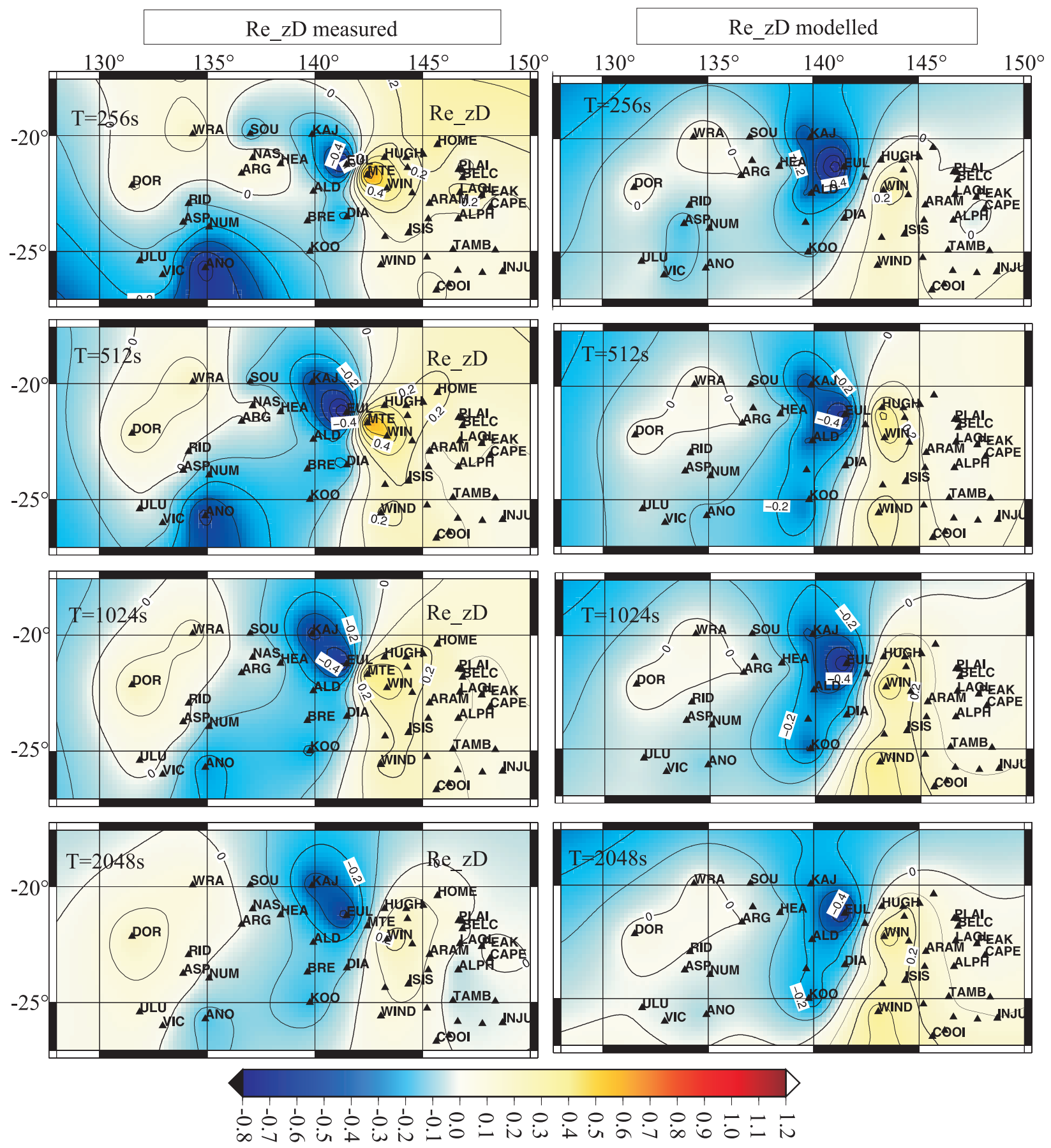

Figure 5.6: Measured and modelled real parts of the $z_{D}$ perturbation tensor elements at four different periods compared. Sites used for the interpolation are labelled on the map. 

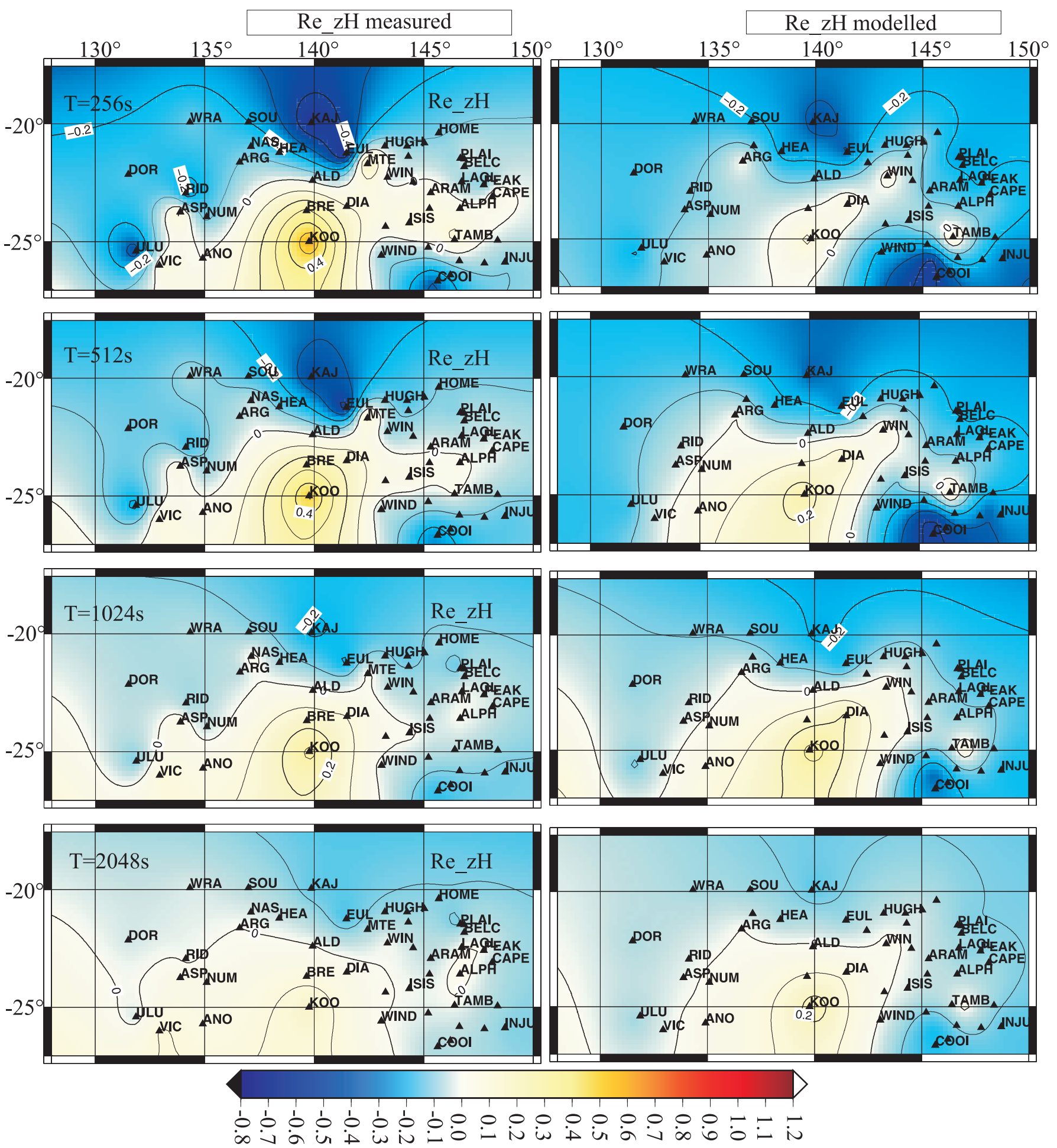

Figure 5.7: Measured and modelled real parts of the $z_{H}$ perturbation tensor elements at four different periods compared. Sites used for the interpolation are labelled on the map.

To be very certain that the conductor that characterises the $z_{D}$ perturbation tensor el- 
ement extends into the mantle, a second model with the conductor limited only to the crust was used. That is to say the only difference between model 1 and model 2 is that the conductor along longitude $142.5^{\circ} \mathrm{E}$ is limited to the crust in model2. Compare figures 5.2 with 5.8. 


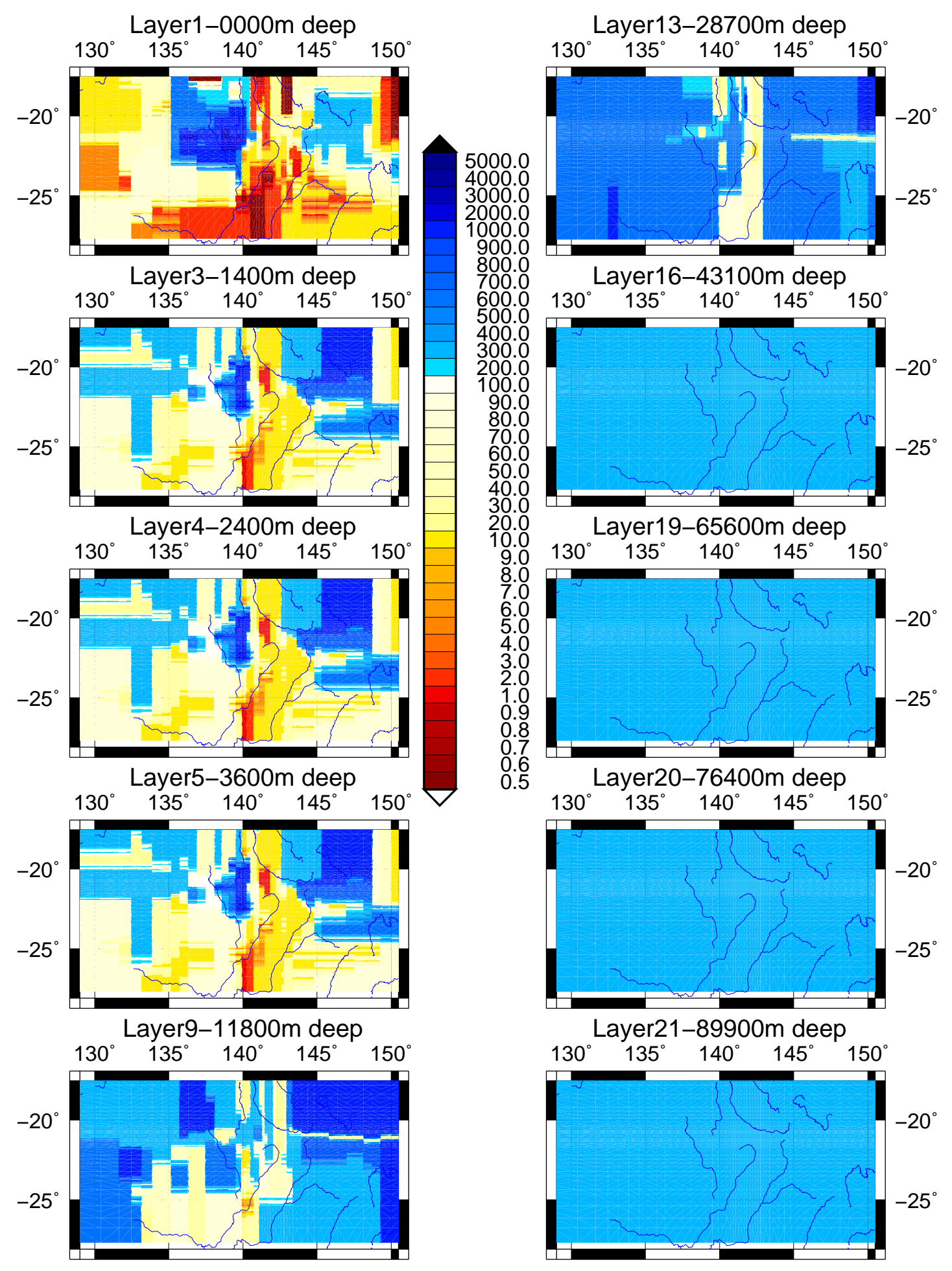

Figure 5.8: Resistivities of some layers of model2, same layers as in the final 3D forward model (model 1) of the project's dataset. 
After analysing model 2, it was observed that it did not fit the measured data well compared to the first model. It was observed as follows: (i) In the second model, the magnitude of the $z_{D}$ perturbation tensor element at long periods for sites west of longitude $142.5^{\circ} \mathrm{E}$ were smaller compared to the measured ones and those in the first model. In particular, at site EUL, the site that forced us to extend the conductor to greater depths in an attempt to fit the $z_{D}$ perturbation elements at long periods with the measured one, the $z_{D}$ perturbation elements of model 1 fit the measured data far much better than those of model 2. Figure 5.9 shows this comparison for the site EUL. Observe that at periods of $8196 \mathrm{~s}$ and $16393 \mathrm{~s}$, the $z_{D}$ perturbation tensors of model 1 and those of the measured data fit perfectly. The discrepancy between the two models is clearly visible from figure 5.9. Values of the $z_{D}$ perturbation tensor elements of other sites (KAJ, ALD, DIA and KOO) close to and west of longitude $142.5^{\circ} \mathrm{E}$ for the two models and the measured data are compared in table 5.1 below. One can clearly observe that $z_{D}$ values of modelb1 fit the measured data better than values from model 2 (ii) It was also observed that the $h_{H}$ perturbation tensor elements for sites KAJ and EUL in the second model became slightly positive at long periods instead of being negative as in the measured data and in model 1. This is due to the fact that in the second model, in the absence of a strike direction at long periods, the currents try to redistribute and flow in both directions, that is in north-south

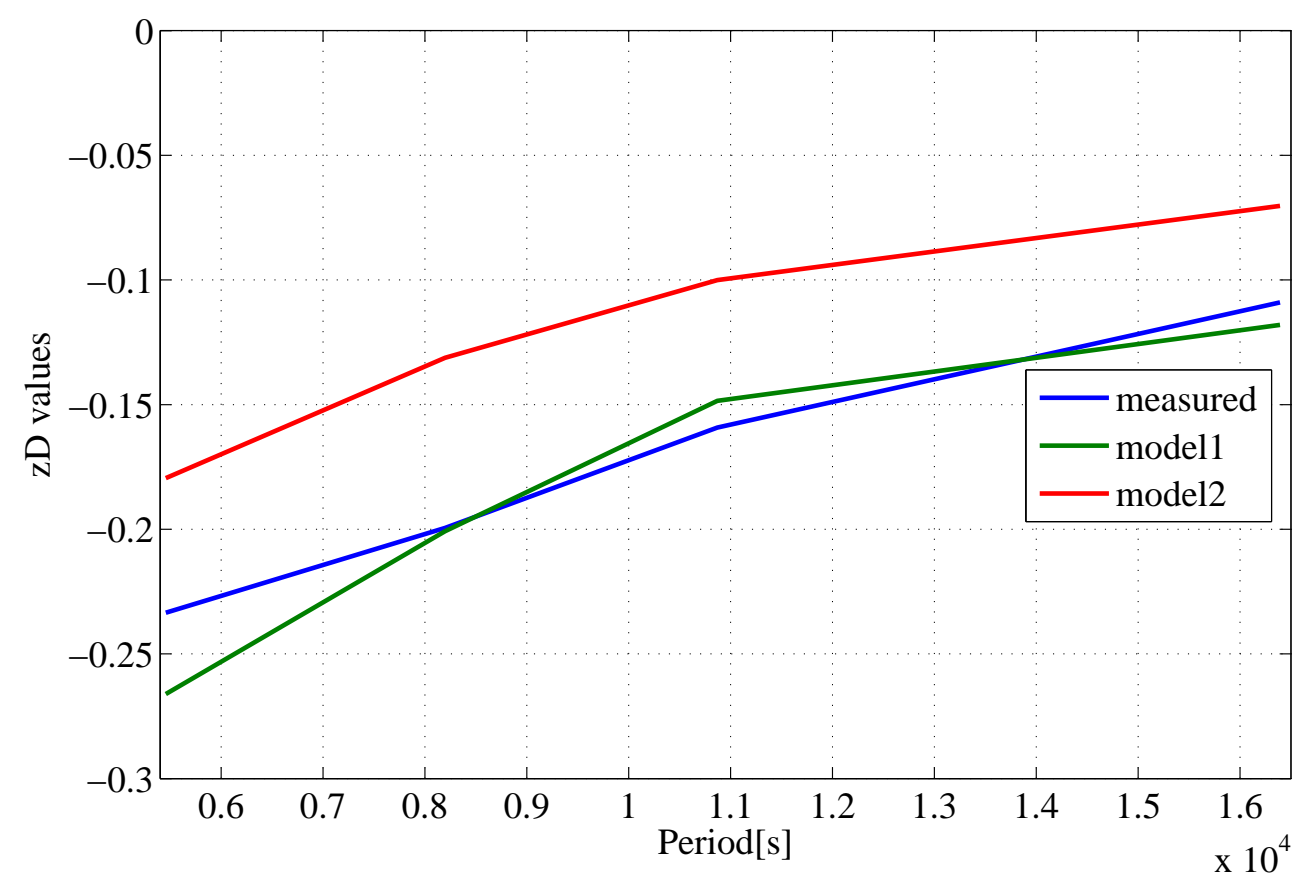

Figure 5.9: Modelled real parts of the $z_{D}$ perturbation tensor elements of site EUL for models 1 and 2 plotted on the same axes with the measured values at long periods for comparison.

There is clearly a significant offset between $z_{D}$ values between model 2 on one hand and model 1 and measured data on the other hand. 


\begin{tabular}{|l|l|l|l|}
\hline \multicolumn{4}{|c|}{ period:5464 s } \\
\hline Site & $z_{D}$-measured & $z_{D}$ from model1 & $z_{D}$ from model 2 \\
\hline KAJ & -0.2212 & -0.1206 & -0.0839 \\
\hline DIA & -0.0758 & -0.0857 & -0.0124 \\
\hline ALD & -0.0800 & -0.1245 & -0.093 \\
\hline KOO & -0.1043 & -0.1558 & -0.136 \\
\hline \multicolumn{5}{|c|}{ period: $8192 \mathrm{~s}$} \\
\hline KAJ & -0.1671 & -0.0877 & -0.0598 \\
\hline DIA & -0.0805 & -0.0577 & -0.0045 \\
\hline ALD & -0.0843 & -0.0880 & -0.0665 \\
\hline KOO & -0.1040 & -0.1099 & -0.0959 \\
\hline \multicolumn{5}{|c|}{ period: $10870 \mathrm{~s}$} \\
\hline KAJ & -0.1690 & -0.0679 & -0.0479 \\
\hline DIA & -0.1045 & -0.0431 & -0.0015 \\
\hline ALD & -0.1035 & -0.0667 & -0.0521 \\
\hline KOO & -0.0940 & -0.0850 & -0.0750 \\
\hline \multicolumn{5}{|l|}{ period: $16393 \mathrm{~s}$} \\
\hline KAJ & -0.1675 & -0.0501 & -0.0346 \\
\hline DIA & -0.1091 & -0.0255 & 0.0010 \\
\hline ALD & -0.0713 & -0.0481 & -0.0375 \\
\hline KOO & -0.0714 & -0.0552 & -0.0519 \\
\hline
\end{tabular}

Table 5.1: Table to compare $z_{D}$ values at different periods for sites west of and closed to our stipulated Tasman line.

The general trend is that at all periods in the table 5.1 , real $z_{D}$ values in column 2 (model 1) fit values in column 1 (measured values) much better than values in column 3 (model 2). This gives more credit to our first model. For site DIA (model 2), at a period of 16393 $\mathrm{s}$, the $z_{D}$ value loses trend completely and swings from negative to positive, a strong indication that model 2 does not satisfy the measured data. Also important to note is the fact that at all periods evaluated above, the magnitudes of $z_{D}$ values for model 2 are smaller than those of model 1 . This is a function of the strength of the conductor centred at longitude $142.5^{\circ} \mathrm{E}$. It is therefore concluded that model 1 is a better model for the dataset. The implications for this conclusion will be discussed later. We then proceeded to evaluate the GDS strike angles of model one and compared them with the measured ones as shown below. 


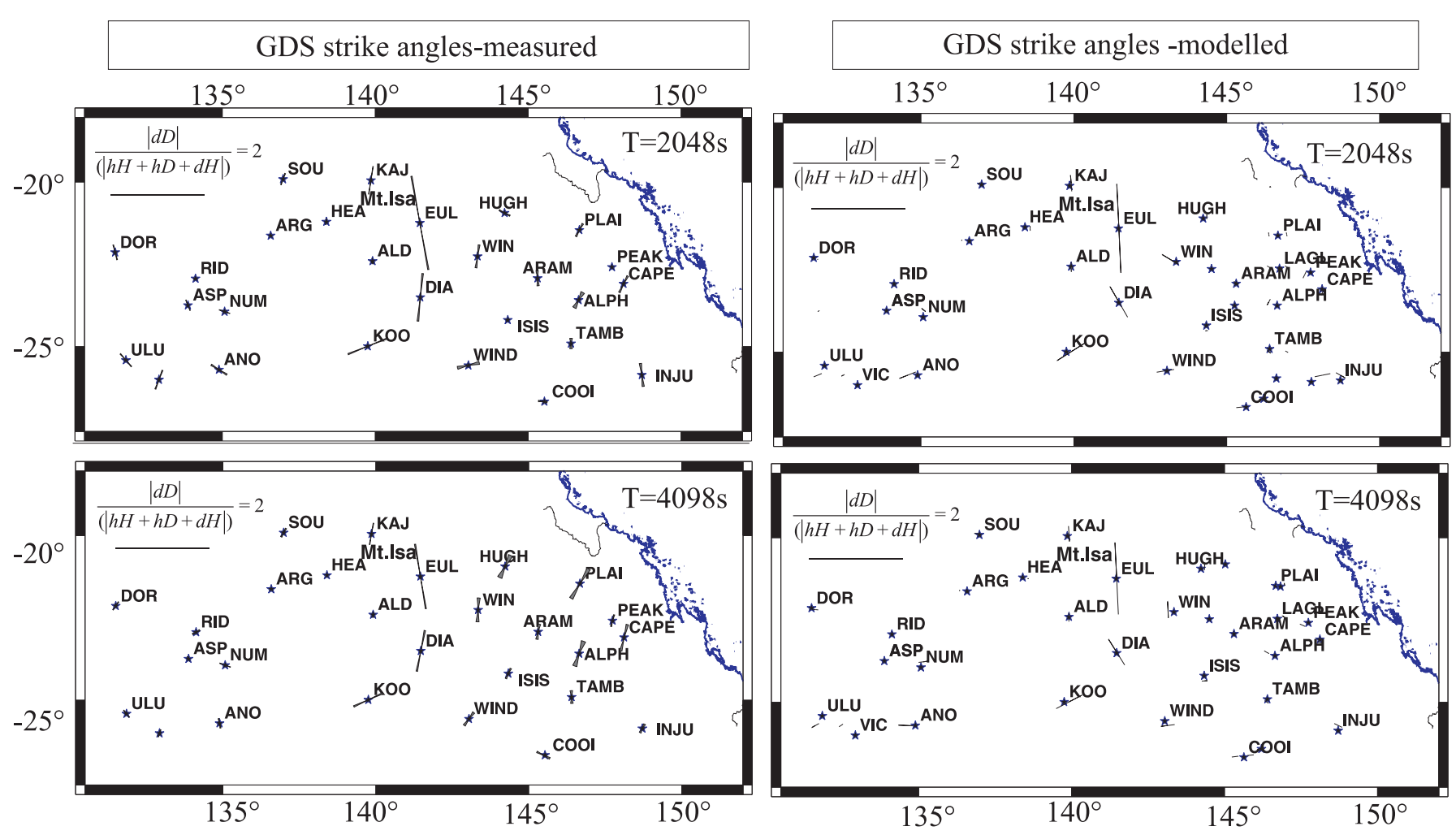

Figure 5.10: Measured and modelled gds strike angles for the periods shown in the figure.

From figure 5.10 above, one can see that the model has reproduced our measured GDS strike angles. There is an especially good match between the measured and calculated GDS strike angles at sites KAJ, EUL, DIA, KOO and WIND, except at WIN. These are sites that fall along the conductive band like structure running north-south of our study area. This is clear indication that our model gives a very good representation of the conductivity structures of our study area and will enable us to give an accurate interpretation of the data.

\subsubsection{MT results}

As earlier mentioned, to avoid the static shift effect, the model targets to reproduce the MT phase of the data. Figures 5.11 and 5.12 below display a comparison between measured MT phases (xy and yx components) and the modelled ones for different periods. Looking at figure 5.11, we are quite satisfied with the strong match between measured and modelled phases of the E-polarization. Most contour lines are reproduced by the model to at least $80 \%$ which is very satisfactory. However, the phases of the B-polarization (yx) show a misfit especially arround the site KAJ which has extreme phase values. The mismatch is an indication that a more complex conductivity structure is present within this region which is not represented properly in the model. This may also be due to our large grid size that may not resolve fine structures in this polarization. Given also the task 
involve in using one model to produce both MT and GDS characteristics, it was difficult to vary the model parameters to reproduce the extreme phases without altering the GDS characteristics which were already very good as demonstrated above. This mismatch alone is not a major concern for the interpretation of the data, especially regional structures that are the main target. 
5.2 Results from the model compared with measured data

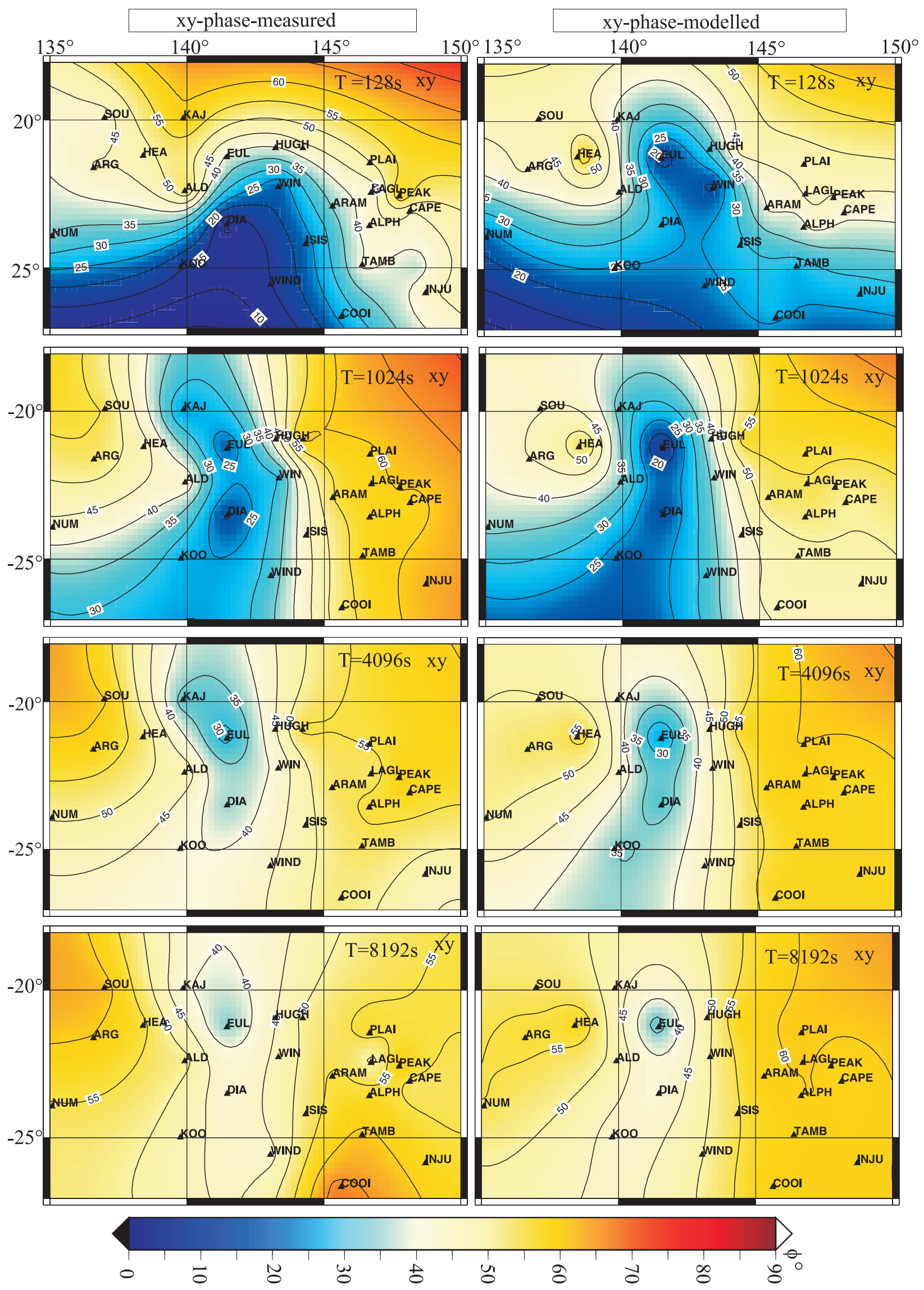

Figure 5.11: Measured phase responses (xy-component) compared with the modelled ones at four different periods shown in the diagrams. 


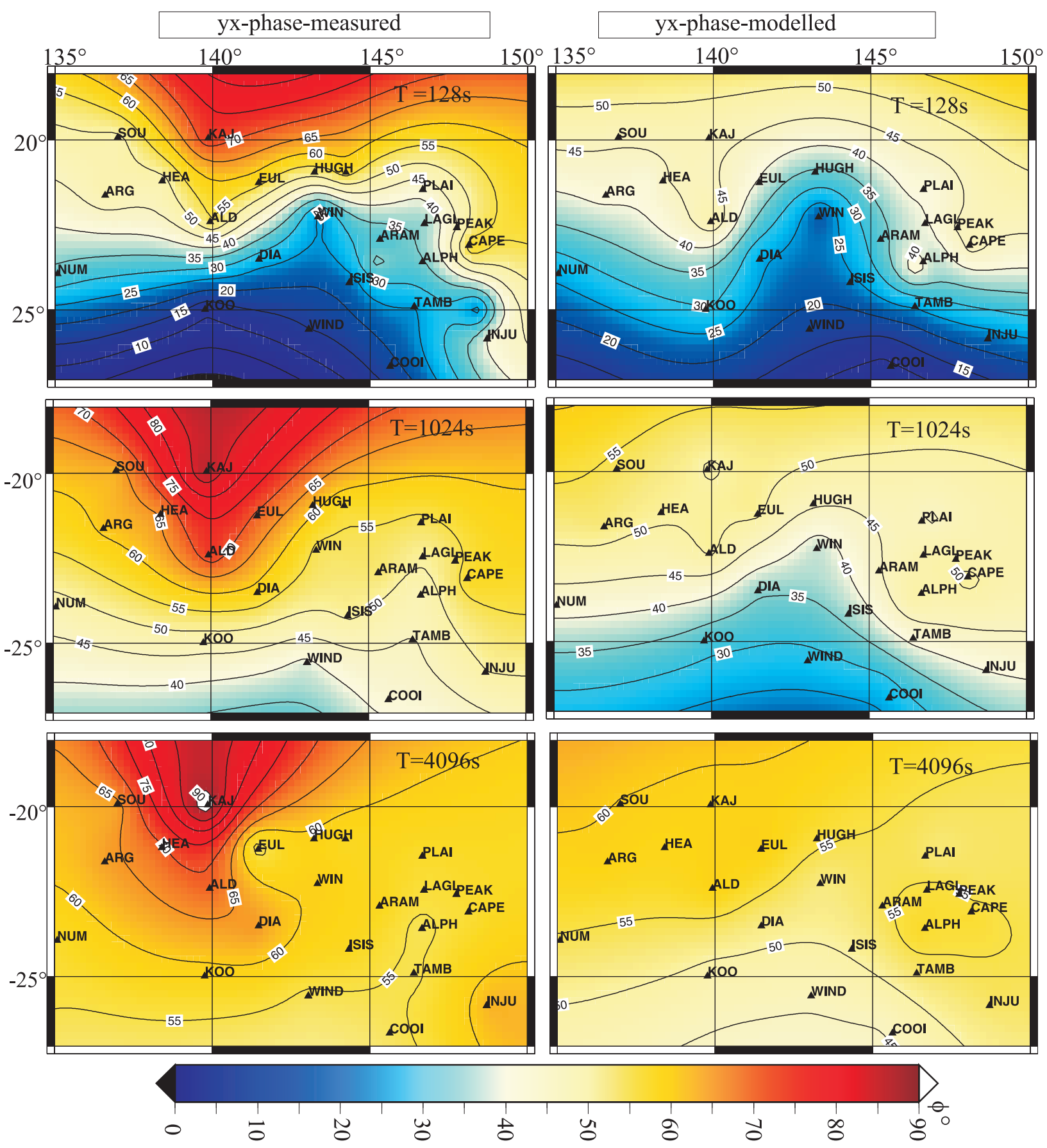

Figure 5.12: Measured phase responses (yx-component) compared with the modelled ones at three different periods shown in the diagrams.

Another important parameter obtained from the modelled MT data is the induction vector. Figure 5.13 compares the measured induction vectors with the calculated ones for four periods. The periods plotted were chosen to include the periods of maximum induction as previously discussed. 


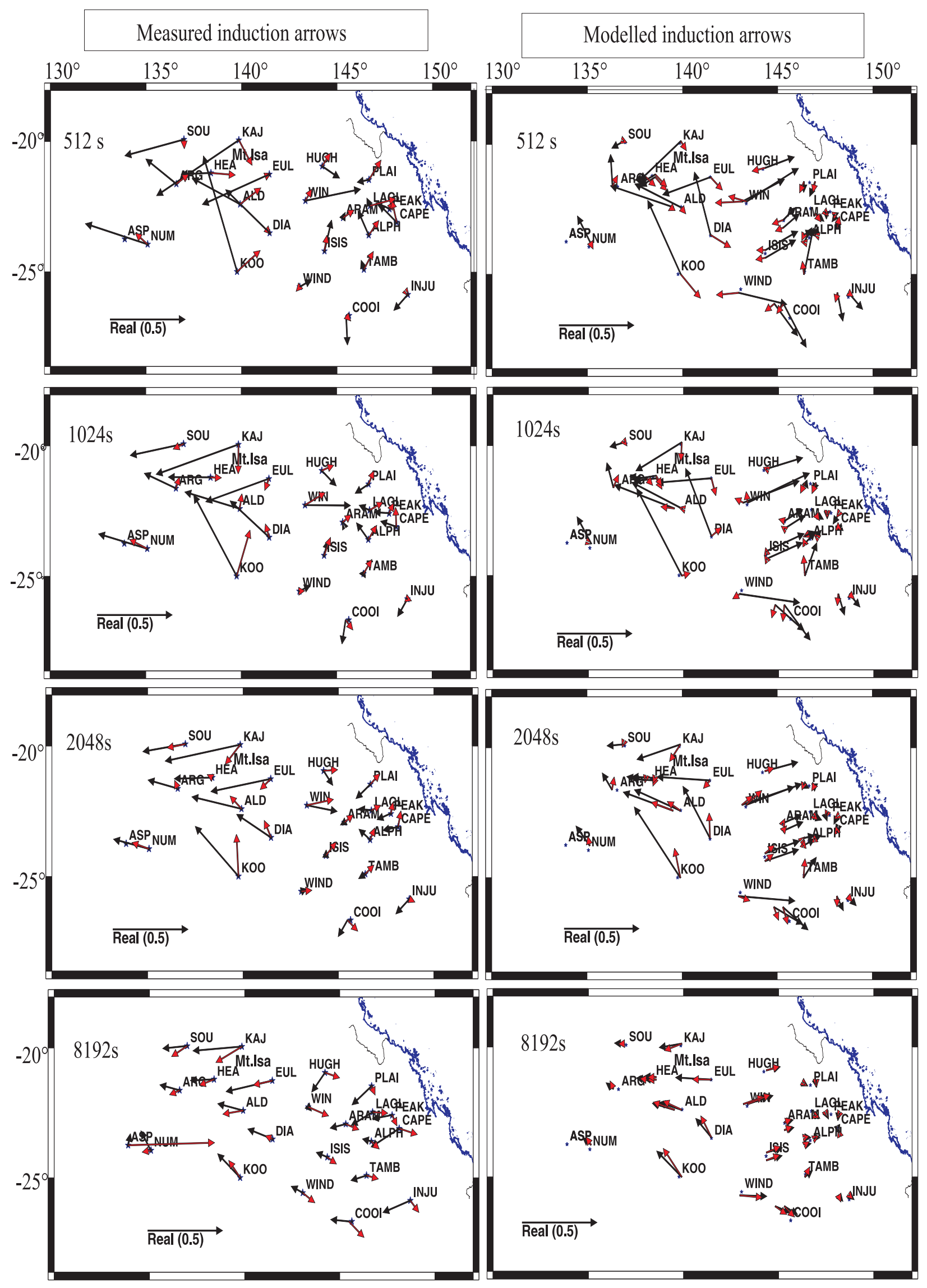

Figure 5.13: Measured and modelled induction vectors compared. 
A very good fit is seen at almost all sites for the periods selected. The model reproduces the measured induction arrows in both magnitude and direction. Interesting and exciting is the fact that the model reproduced the induction arrow at site KOO also both in magnitude and in direction for real and imaginary arrows. This intriguing result is a strong indication that the east-west conductor joining $\mathrm{KOO}$ and $\mathrm{COOL}$ predicted from the measured data and included in the model is well represented with the right conductance and at the right position.

The fact that induction arrows are reproduced along the transect between longitudes $139^{\circ}$ and $143^{\circ} \mathrm{E}$ indicates that the Carpentaria conductor and the conductor constituting the Tasman Line of the model have the same conductance values as it would need to reproduce anomalous magnetic fields observed in the region. The same holds for the south west Queensland anomaly.

The good match between modelled and measured induction vectors to the extreme east of the study area is an indication that the Coral Sea was represented with the right conductance that generates the same anomalous vertical field as the actual sea.

\subsection{Structural \& geological implications of the model}

Important geological regions mapped out by the measured data and confirmed by the model include: (i) Conductive sediments of the Eromanga Basin, (ii) Resistive structures in and around the Mt. Isa block, whose resistivity decreases with depth, (iii) a conductive band that represents (follows the trends of) the Carpentaria anomaly and part of the Tasman Line (iv) moderate resistive Phanerozoic rocks of eastern Australia compared to more resistive Precambrian rocks to the west, (v) Part of the southwest Queensland conductivity anomaly with an east-west strike direction. These regions are shown on figure 5.14 below. 


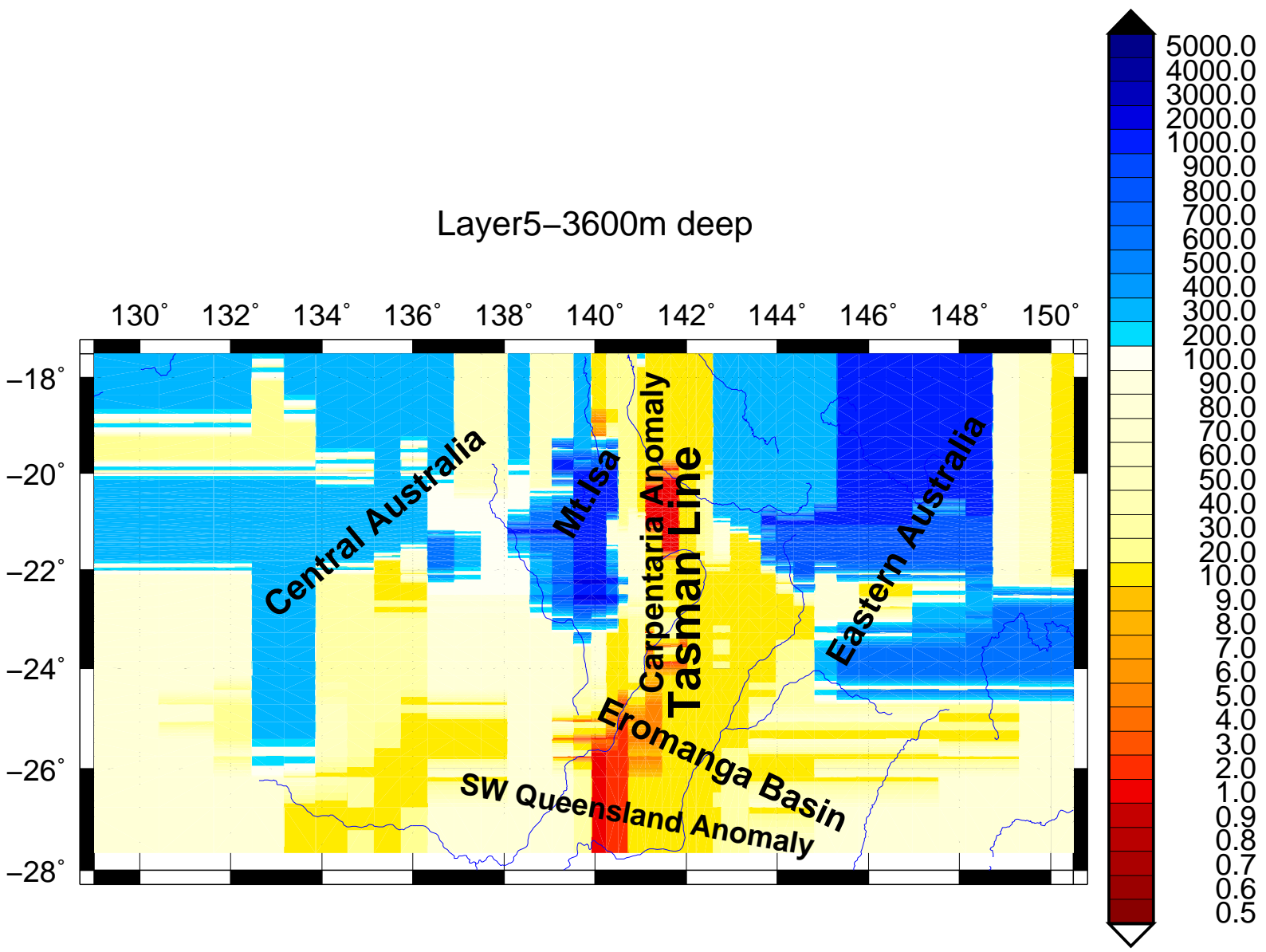

Figure 5.14: Geological regions identified by the data and model.

\subsubsection{Sediments of the Eromanga Basin}

The result of the 3D forward model indicates high conductivity in the upper crust in the portion of the model that falls within the Eromanga Basin. The resistivities modelled for the sediments of Eromanga basin in this project lie between 1 and $20 \Omega \mathrm{m}$ and the depth of the sediments is $(2500 \pm 500) \mathrm{m}$. Lilley et al. (2003) obtained resistivity values between 1 and $10 \Omega \mathrm{m}$; Spence \& Finlayson (1983) determined values of $<5 \Omega \mathrm{m}$ from magnetotelluric measurements; Woods \& Lilley (1980) published in the range 1 to $20 \Omega \mathrm{m}$; Whiteley and Pollard (1971) using magnetotelluric and DC-resistivity approach obtained values in the range 1.5-55 $\Omega \mathrm{m}$ and McDougall (1996) using magnetotelluric and time-domain published values in the range 1-10 $\Omega \mathrm{m}$. These low values of resistivity reflect the highly conductive sediments of the Eromanga Basin that covers much of central Australia.

As already discussed in section 4.3, the Eromanga basin falls in region 4 of the study area. At short periods, the phases measured and modelled for sites in this region are between $10^{\circ}$ and $15^{\circ}$ and then increases with period, indicating that resistivity increases with depth in this region. To explain the high conductivity of the sediments, we first examine the general factors that control electrical conductivity of rocks. The conductivity of water-bearing rocks depends on: 
- Porosity and the pore structure of the rock

- Amount of water (saturation)

- Salinity of the water

- Temperature

- Water-rock interaction and alteration

- Pressure

- Steam content in the water

The most important parameters controlling the conductivity of rocks are porosity, temperature, salinity and water-rock interaction. It is important to note that the rock matrix itself is normally an insulator. The conductivity of sedimentary rocks is generally dominated by the conducting pore fluids (porosity). Porosity, the ratio between the pore volume and the total volume of a material is basically grouped into three types: Intergranular, the pores are formed as spaces between grains or particles in a compact material (sediments). Joints-fissures, a net of fine fractures caused by tension and coolling of rock (igneous rocks, lava). Vugular, big and irregular pores, formed as material is dissolved and washed away, or pores formed by gas (volcanic rocks, limestone). Pore spaces must be interconnected and filled with water in order that fluid conduction occurs. In all types of porosity there are larger voids, called storage pores, and finer connecting pores.

For the sediments of the Eromanga Basin, intergranular porosity is implied. Since the Eromanga Basin is a subdivision of the Great Artesian Basin, central Australia's largest water reservoir(GABCC, 2000), the Eromanga Basin therefore has a high fluid content. In addition, the uppermost Jurassic-Cretaceous Eromanga Basin sequence contains flowing aquifers (Spence \& Finlayson, 1983). According to the empirical law, called Archie's law (Archie, 1942), if ionic conduction in pore fluid dominates other conduction mechanisms in rocks, the resistivity of water-bearing rocks varies approximately as the inverse square of the porosity.

$$
\rho=\rho_{w} a \phi_{t}^{-n}
$$

where $\rho$ =bulk (measured) resistiviy; $\rho_{w}=$ resistivity of the pore fluid; $\phi_{t}=$ porosity in proportions of total volume; $\mathrm{a}=$ empirical parameter, varies from $<1$ for intergranular porosity to $>1$ for joint porosity, usually around 1 ; and $\mathrm{n}$ is a cementing factor, an empirical parameter which controls the pore geometry and typically ranges between 1 (crack like pores) and 2 (spherical pores common for crystalline rocks). The small modelled resistivities in the upper crust, in the range 1 to $20 \Omega \mathrm{m}$ in the Eromanga Basin can be explained in terms of the conducting pore fluids in the Basin (equation 5.1). The Eromanga Basin which is younger than the Georgina Basin is more conductive since resistivity increases with age due to the closure of pore spaces. To obtain the small phases less $15^{\circ}$ as in measured data, for sites in the Eromanga basin, a top thin highly conductive layer of conductance (400 S) was included in the model followed by a thicker moderately resistive layer of conductance $(20 \mathrm{~S})$. 
Since electromagnetic methods give a volume sounding due to diffusion of electromagnetic waves, it implies that conductivity boundaries cannot be exactly resolved. The thickness of the sediments from the model is estimated to be in the neighbourhood of $(2500 \pm 500) \mathrm{m}$ towards the centre of the basin. The uppermost layers of the basin are characterised by a 1D conductivity structure. For sites which fall in the basin and which are part of region 4 (figure 4.4), along the Tasman Line, the conductivity structure cannot be described as $1 \mathrm{D}$; the model proves it to be 3D especially for periods above $100 \mathrm{~s}$.

\section{Interpretation of the uppermost zones of the Eromanga basin}

The low average resistivities modelled in the uppermost zones of the Basin correspond to the sediments of the Jurrassic-Cretaceous Eromanga Basin sequence. The high average conductivities reflect high mean porosity (conductivity of rocks depends on porosity) and saturations levels (conductivity of near surface rocks is a function of the water content of the rocks; Australia is often flooded and the water turns to settle in the low Basins). In the basin, the upper boundary of the saturation zone occurs at depths of less than 20 m (Spence \& Finlayson, 1983). As already mentioned above, the base of the sequence of high conductivity sediments lies at an average depth of about $2.5 \mathrm{~km}$.

Another feature of this upper zone is the occurrence at some sites, of average resistivities that are anomalously lower than the mean for the zone. This occurs at sites DIA and KOO; these sites are along the anomalously conducting band like structure, the stipulated Tasman Line. Therefore conductivity at these sites is influenced by both the conductive sediments and the anomalies that define the Tasman Line and the southwest Queensland anomaly (site KOO). Therefore the sediments and conductive material along the Tasman Line form a suitable path for electric conduction; this is particularly the case since the sedimentary layers and the Tasman Line are connected to the open oceans to the north and in the south.

The general conductivity trends in rocks have been discussed above. In the central Eromanga Basin the sediments are part of a large flowing Artesian Basin and there is a considerable thickness of these sediments under each site. In partially-saturated sediments, Keller and Frischknecht (1970) gave another empirical relationship different from equation (5.1) between resistivity and porosity as follows:

$$
\rho_{p s}=\rho\left(S_{w}\right)^{-n}
$$

where $\mathrm{n}$ is a constant and can be equal to 2 (Wyllie, 1957), $S_{w}$ is the fraction of the pore space filled with water or conducting fluid and $\rho_{p s}$ is the resistivity of the partially saturated sample. By analysing equation (5.2), it will lead to the conclusion that in partially-saturated sediments the influence of the water-content factor is orders of magnitude greater than that due to the conductivity of water and the way the water is distributed (connectivity) in the rocks. However, when the sediments are fully saturated, equation (5.1), that is variation in the conductivity of the water itself becomes the main source of resistivity variation. This is expected to be the situation in the central Eromangaga Basin, where we assume the porosity and saturation level to be nearly uniform from site to site except for sites that fall on the path of the Tasman Line. This can easily explain the $1 \mathrm{D}$ conductivity structures in the crust under many sites in the Basin. 
Why do some sites in the Basin apart of those that fall along the Tasman Line e.g. TAMB deviate from a $1 \mathrm{D}$ conductivity in the crust? TAMB falls in eastern Australia that is made up of different Orogens and may fall on the boundary between the Thomson and new England Orogens. On the other hand, the conductivity of groundwater is controlled by the concentration of dissolved salts and the cation exchange capacity of host rock. Both these processes are temperature and time dependent. Local vertical movement along faults of hot groundwater from great depths can increase near-surface temperatures. Faulting may also cause increased salinity, due to long periods of chemical interaction between water and rocks (Spence \& Finlayson, 1983). These factors may cause the non uniformity of subsurface conductivity at some sites, therefore explaining the deviation from $1 \mathrm{D}$ conductivity at TAMB.

According to Senior \& Habermehl (1980) comparatively small fault-displacements can obstruct several aquifers and cause stagnation zones. These displacements are more likely to produce an effective seal of disconnected aquifers than larger movements, which may result in a broad fracture zone, permitting substantial leakage.

The significantly small average resistivity values in the near-surface zones at sites TAMB, WIND, COOL and WIN (figures B.2 and B.3 in appendix B) could be due to local increases in porosity (from equation 5.1, resistivity decreases exponentially with porosity) and/ or due increased salinity resulting from long periods of stagnation. Senior \& Habermehl (1980) associated such stagnation zones with hydrocarbon entrapment.

\subsubsection{Resistive structures in and around the Mt. Isa block}

The Mt. Isa Block has a diverse geological structure with various fault systems and intrusions. The unique structures of the area are associated with multiple tectonic events e.g. compression, extension and intrusion. The geological architecture began to build up during the Barramundi Orogeny (a compressive orogenic event that marks the deformation, metamorphism and cratonisation of the earliest Proterozoic era, chapter 3) that is speculated to have taken place between 1900-1870 Ma (Betts et al., 2006) and continued around 1600 Ma by a period of multiple transient extensional events that switched to intense crustal thinning, which caused the interruption of basin development (Betts \& Giles, 2006). The period ended in the onset of the Isan Orogeny (in about 1600-1500 Ma e.g. Page and Bell (1986), O'Dea et al (1997), Betts et al. (2006) and Giles et al. (2006)).

Betts and Giles (2006) described a plate margin located along the eastern north Australian craton formed during a protracted history of continental break-up and ocean basin formation between 1800 and 1650 Ma. During the Isan Orogeny, west-dipping subduction along this margin resulted in the development of a collisional orogenic belt that is now preserved in the Mt. Isa Inlier (Betts et Giles, 2006). The west-dipping subduction is supported by results from seismic refraction data indicating a west-dipping high velocity layer in the entire Mt.Isa Block (MacCready, 2006). The intrusive events characterising this region indicate the presence of resistive igneous rocks resulting from magma that cooled and solidified within the crust. The intrusions and fault systems should of course determine the conductivity character of the Inlier. 
From apparent resistivity curves the measured data revealed Mt. Isa block as highly resistive compared to other sections of the study area. The modelled resistivities lie in the

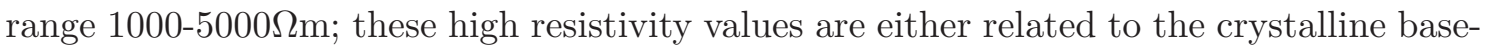
ment that are common to Proterozoic crust (Boerner, 1999) or to Igneous rocks resulting from intrusion in the region. The crust of the region is highly resistive and thereafter resistivity decreases with depth. The highly resistive crust implies either a very thin covering of sediments or no sediments at all. In regions of the block where there are completely no sediments, there is a high probability of the presence of plutons (large bodies of magma that solidify underground before they reach the surface of the crust).

It was already mentioned that the resistivity values in the region decrease with depth; the rate of decrease is not the same for the entire block. East of the block (close to our postulated Tasman Line) there is a significant increase in conductivity in the north-south direction beginning at depths of about $10 \mathrm{~km}$ (figure 5.3, see resistivity values between longitudes $139^{\circ}$ and $140.5^{\circ}$ ). In the $3 \mathrm{D}$ forward model, an anisotropic layer was modelled from $10 \mathrm{~km}$ downwards to fit the strong MT split and the positive $d_{D}$ anomaly, strongest at a period of $1000 \mathrm{~s}$ for sites in eastern Mt. Isa ( e.g. KAJ and ALD). The negative $h_{H}$ anomaly and the positive $d_{D}$ anomaly mean a north-south strike direction. The significant increase in conductivity in the basement of eastern Mt. Isa suggests an extension of the conductivity anomaly (Carpentaria anomaly) described as occupying region 3 (figure 4.4) of the survey area to Mt. Isa. At higher depths (from about $200 \mathrm{~km}$ ), judging from our forward model, our GDS results are homogeneous in relation to other portions of the survey area suggesting a uniformity in the lower part of upper mantle conductivity for Australia. This is in agreement with results from Quiet-day ionospheric currents and their application to upper mantle conductivity studies by Campbell et al. (1998), where they used a regional spherical harmonic analysis (SHA) to separate the internal and external field contributions to the Sq variations.

\subsubsection{Carpentaria conductivity anomaly}

In section 3.5, we gave an account of the Carpentaria conductivity anomaly (also shown on figures 3.4 and 3.5), a major element in the electrical conductivity structure of the Australian continent (Lilley et al., 2003). Previous information such as its strike direction, position and lateral extent was significantly helpful for our initial 3D forward model. Results from AWAGS indicated the strike of the Carpentaria conductivity anomaly to be north-south. This is supported by our induction arrows (figure 4.9), the outcome from contour plot of the $d_{D}$ perturbation tensor elements (figure 4.13) and GDS strike direction (figure 4.15). The initial 3D forward model to fit the $d_{D}$ perturbation tensor elements revealed the anomaly as a broad conductor spanning hundreds of kilometres between approximately longitude $139^{\circ}$ and $144^{\circ} \mathrm{E}$, running north-south and limited to the crust. Chamalaun et al.(1999) defined the lateral extent of the Carpentaria conductivity anomaly over a distance of more than $1000 \mathrm{~km}$ judging by the reversal in the real induction arrow response.

In an attempt to fit the vertical perturbation tensor element $z_{D}$, we resolved a slightly distinct and narrow conductor centred on longitude $142.5^{\circ} \mathrm{E}$ (figures $4.10 \& 5.14$ )and which extends into the mantle (figures 4.9, 5.2\& 5.3), and as discussed for model 1. That means 
the depth extent is at least $80 \mathrm{~km}$. One cannot accurately determine the depth extent of the anomaly because magnetotellurics provides poor resolution of resistive regions beneath conductive zones. This result from electromagnetic induction indicating that the depth of the 'Carpentaria conductivity anomaly' extends into the upper mantle is new and sheds more light on the concept of the Tasman Line. Nevertheless, this is in conformity with results from seismic observations which extended the depth of the anomaly into the upper mantle (Lilley et al., 2003). The anomaly is linear, extending north into the Gulf of Carpentaria; deduced from extrapolation. Chamalaun et al. (1999); Lilley et al. (2003) have previously concluded that the anomaly extends into the Gulf of Carpentaria. To the south, it extends several kilometres and intersects with another anomaly delineated by this model striking approximately east-west and linking sites KOO and COOL (figures 4.10 \& 5.5). The Carpentaria anomaly and/ or Tasmanline crosses this anomaly at site KOO. This anomaly is certainly the southwest Queensland anomaly that links the Flinders Range conductivity anomaly (Chamalaun \& Barton, 1993) to the south.

A lot has been investigated about this major electrical conductivity structure but the relationship between the conductivity anomaly and major tectonic boundaries is still not well understood. The following questions are often asked: What is the cause of high electrical conductance within and beneath the crust? In other words, what is the origin of the anomaly? What is the spatial relationship between the anomaly and tectonic structures in the region? How are the electrical properties of the conductor related to the tectonic evolution of northern Australia? We attempt to discuss these questions based on general causes of enhanced conductivity in the subsurface and our knowledge of the evolution of the region as discussed in chapter 3 .

There is still an ongoing research concerning the origins of high deep-crustal conductivity especially in stable continental crust. Crustal and upper mantle magnetotelluric studies seek physical explanations for zones of enhanced conductivity. Different reasons have been advanced to explain the cause of anomalous conductivity in certain regions. If mineralogy such as metallic sulfides and graphite are present even in small percentages but are well connected, they can give rise to anomalous conductivity (Nover et al., 2005; Adam, 2005; Jödicke, 1992). Nonetheless, the conditions under which graphite is formed from organic carbon makes it rare for it to be formed in the mid-crust under normal conditions. Its formation requires extreme temperatures (greater than $1000^{\circ} \mathrm{C}$ ), unless strain energy from sources such as active rifting and mantle diapirism or collisional events is present to facilitate graphite formation at mid-crustal conditions (greater than $450^{\circ} \mathrm{C}$ ) (Nover et al., 2005). It is assumed that graphite present in the deeper levels of thrust belts and fold, not excluding sutures and fossil shear zones was formed under these conditions. Boerner et al. (1996) attributed elevated deep-crustal conductivity to the deformation and metamorphism of graphitic or sulfidic sedimentary rocks formed in an ocean environment. At the same time, studies have shown that high conductivity anomalies observed within active tectonic margins are rarely due entirely to graphite and metallic sulphides (Telford et al., 1976).

Within active tectonic environments, aqueous fluid and partial melt are considered as major sources of large-scale enhanced conductivity (Wei et al., 2001; Li et al., 2003). Despite present knowledge about the contribution of graphite, aqueous fluid and partial melting to anomalous conductivity, the origin of deep conductivity over geologic time scales 
in stable continental crust continues to puzzle researchers in Earth sciences. Recent studies have shown that subduction zones and continent-continent collisions produce conductivity anomalies (e.g Soyer \& Brasse (2001); Li et al. (2003)) over geological times scales for resasons that are still being debated ( Tauber et al., 2003; Ritter et al., 2003b; Almeida et al., 2005; Boerner et al., 1996). As for the Carpentaria anomaly, Lilley et al. (2003) associated part of its enhanced conductivity to the presence of metasedimentary rocks emplaced in the crust during the subduction of oceanic crust at the margin of the Mt. Isa Block. As described in chapter 4, the location of the Carpentaria conductivity anomaly is within an active continent-continent collision zone and hence its enhanced conductivity distribution may reflect remnants of plate collisions.

As earlier mentioned, the anomaly is linear probably supporting the hypothesis that collisional plate boundaries have an electrical signature in the form of a major linear conductor (Lilley et al., 2003). The Carpentaria conductivity anomaly lies between two crustal blocks (Precambrian west and Phanerozoic east Australia). The presence of two crustal blocks with different resistivities separated by a deep electrical discontinuity suggest a collision model (Ritz \& Robineau, 1986). This supports the collisions and break ups / subductions described by Betts et al.(2006) and Betts \& Giles (2006) at the eastern margin of Mt. Isa. It can be proposed with some confident that the Carpentaria conductivity anomaly was formed during continent-continent collisions that characterised the evolution of the Mt. Isa Block. Lilley et al. (2003), interpreted and associated the enhanced conductivity of the Carpentaria anomaly with the collisional process at the margin of the Mt. Isa Block.

The ancient cratons (North, West and South Australian Cratons) have episodically collided and assembled in periods of Orogenesis (e.g Barramundi Orogeny between 1900-1870 Ma (Betts et al., 2006) or Isan Orogeny in about 1600-1500 Ma (Giles et al., 2006)) to form a supercontinent called Rodinia. The large landmass of Supercontinents become unstable probably because such a large single landmass acts as a thermal lid that limits the escape of the Earth's internal heat leading to a break-up into more stable land masses (Cengage, 2003, 2006). Giles et al. (2004) described a series of amalgamation, break-up and reattachment of the major Australian cratons between 1500 and 1100 Ma. Break-up of the Supercontinent Rodinia is said to have occurred around $780 \mathrm{Ma}$ (Myers et al., 1996; Giles et al., 2004). The Carpentaria anomaly must have been formed during one of these events.

\subsubsection{Tasman Line}

Many geophysical methods have been used to map the Tasman Line e.g. Gravity, magnetic and seismic methods. The disadvantage of gravity and magnetic methods is that mantle structures cannot be mapped since these methods can only resolve geophysical information limited to the crust. The Seismic method can resolve mantle structures and therefore could be a good candidate to use and delineate the Tasman Line. But the method has a limitation because it does not say much about the region (neutral marker) between two zones with different shear wave speeds. In the case of the Tasman Line, the neutral marker is the Tasman Line that separates two zones of different wave speeds; therefore the method does not say much about the structures along the Line itself. It tells us the wave speed on the opposite sites of the line only and if the line is broad probably due to deformation 
over geological times, its resolution would be poor. Progressive accretion of the eastern part of Australia through a sequence of subduction and rifting events could be expected to have modified the original Tasman Line (Kennet et al., 2004) and therefore broadened it.

To understand why the seismic method may not offer a better resolution of the Tasman Line, we revisit the insightful fluid porosity model by Marquis and Hyndman (1992) which established a seismic velocity-electrical resistivity correlation as a function of pore fluid. According to this model, a high seismic velocity zone also has a high electrical resistivity. Both seismic velocity and electrical resistivity are functions of fluid porosity. Because of loss of pore fluid with time, both lower crustal resistivity and seismic velocity values from Precambrian areas are higher than those for Phanerozoic regions. What does this mean to us? It tells us that: (i) if the main factor enhancing conductivity along the Tasman Line is not pore fluid, we will always have high seismic velocity in the Precambrain west due to the fact that Precambria regions have a small volume of pore fluid (compared to Phanerozoic regions) and lower seismic velocity in the Phanerozoic east due to a higher volume of pore fluid and the exact boundary of contrast between Precambrian west and Phanerozoic east may not be resolved. (ii) If the main factor enhancing conductivity along the Tasman Line is pore fluid, then the lowest seismic velocity corresponding to the lowest electrical resistivity should be recorded along the Tasman Line. However, this lowest seismic velocity along the Tasman Line compared to that to the west and east has not yet been reported so far. A high conductivity zone in the upper mantle should correlate with a low velocity. This shows that if we rely on seismic methods, the position of the line will depend on the interpretation of data and cannot be unique.

Selway, Heinson \& Hand (2006) have also demonstrated the weakness of the seismic method in the resolution of fundamental crustal boundaries when they imaged a crustalscale conductivity contrast across a steeply-dipping boundary in Central Australia using MT Sounding and compared their results with those obtained from seismic studies. They imaged steeply-dipping conductivity interface separating the North Australian Craton from a younger accreted terrane which previous seismic reflection methods failed to delineate. Nonetheless, we have used results obtained in the area e.g by Kennet et al. (2004) from seismic analysis to justify our electromagnetic induction results which extend the the contrast between Precambrian west and Phanerozoic into the mantle.

Electromagnetic induction methods offer a more satisfactory way to resolve the Tasman Line (a fundamental crustal bounary between the young crust of eastern Australia and Proterozoic central Australia) as an enhanced conductivity boundary between the old resistive Proterozoic west and younger more conductive eastern Australia. This is because the method can resolve the contrast between the two provinces and to great depths than than other geophysical methods (Selway, Heinson \& Hand, 2006).

In the study area, the Tasman Line coincides with the Carpentaria anomaly in north Queensland (figure 5.14). As already mentioned, the Carpentaria anomaly is broad, spanning at least $400 \mathrm{~km}$ west to east and therefore one needs extra care to extract the Tasman Line from it. The vertical perturbation tensor, $z_{D}$ offers a better way to constrain the exact position of the Tasman Line (figure 5.6). It has enabled us to position the line at $(142.5 \pm 0.5)^{\circ} \mathrm{E}$, east of Mt. Isa in Queensland. The position concur well with that 
described by Cengage $(2003,2006)$ when he averred that the Mt. Isa Belt in Queensland, Australia, is trucncated by a rifted margin formed during the breakup of Rodinia.

The electromagnetic induction results delineate it as a boundary between two crustal fragments to a depth of at least $80 \mathrm{~km}$, a level already in the upper mantle. Kennet et al. (2004) observed a relatively sharp transition in wave speed between Proterozoic west and Phanerozoic east with the Tasman Line as a neutral marker right down to depths of $225 \mathrm{~km}$. They observed a very strong contrast in the mantle structure beneath Australia with lowered shear-weave speeds along the eastern margin as compared with elevated wave speeds in the centre and west, which extend to at least $200 \mathrm{~km}$ depth. Such varying wave speeds extending into the mantle across a boundary can only be associated to rocks of different ages on opposite sites of the boundary. In Queensland, their dividing line was approximately $140^{\circ} \mathrm{E}$ and they called it the edge of a coherent zone of thick lithosphere that may represent the ancient core of the continent.

In this study the Tasman Line is positioned along longitude $(142.5 \pm 0.5)^{\circ} \mathrm{E}$ (figure 5.14), the discrepancy between this result and that of Kennett et al. (2004) may lie in the resolution of their data. Models of shear-wave speed structure were constructed from the surface-wave tomography based on the assumption that the mantle structure vary smoothly, however, there is a sharp transition in mantle structure across the vicinity of the Tasman Line.

The depth resolution of the Tasman Line is hindered by the coast effect from the east. Since it is the vertical perturbation tensor element, $z_{D}$ that is the main delineator of the Tasman Line at great depths, the coast effect which increases with period and therefore with depth affects the $z_{D}$ perturbation tensor element and hence the resolution of the line at greater depths. At latitude $25^{\circ} \mathrm{S}$, the Tasman Line takes a westerly direction approaching longitude $140^{\circ} \mathrm{E}$. This has some similarities with previous Tasman Lines (figure 3.6). However, the relationship between the Tasman Line and the southwest Queensland anomaly also modelled in this project is not clear. This could be resolved if more magnetotellurics sites were installed further south along the Tasman Line.

The data and model has resolved the Tasman Line to have a distinct conductivity from that of rocks to the west and those to the east of it. It is hard to explain the source of the anomalous conductivity along the Tasman Line that has existed for close to 500 million years. Most mantle anomalies have been interpreted mainly in terms of high temperature and partial melting (Honkura, 1978), since conducting anomalies coincide well with anomalies in heat flow and seismic wave velocities. The Tasman Line conductivity anomaly, although it extends into the mantle, cannot not be interpreted in a similar manner since it is a straight anomaly. To attempt an explanation for the source of enhanced conductivity along the Tasman Line, one will need to first understand how rifting and closure occur.

A popular mechanism of rifting of continents is by mantle diapirism. This is rifting initiated by an uplift or ascent of the more ductile mantle, in particular the asthenosphere (see Popov et al. (1999) for details). The decompression associated with uprise of an asthenosphere diapir leads to melting of peridotites in the mantle to produce alkaline basaltic magmas (Honkura, 1978; Bott, 1995) which are are highly conducting. Asthenosphere is a $1200^{\circ} \mathrm{C}$ hot medium containing a molten phase (Zorin et al., 1995). Therefore this process 
of rifting can introduce connected molten material and basaltic magmas in the rocks of the crust and upper mantle along a rift system. The high temperatures in the lower crust and upper mantle resulting from upswell of asthenosphere also satisfy the condition required for graphitization. Sedimentary and volcanic rocks are also formed in a rift setting. This analysis implies that there is the presence of conductive material along a rift. How these materials still enhance conductivity when the rift closes, e.g. the case of the Tasman Line is not well understood. However, we hypothesize that the conductive materials produced during rifting are buried during the closure of the rift and remain conductive. Korja (1997) noted that when subsequent tectonic processes closed basins, the buried sedimetary material is subjected to high temperatures thereby enhancing eletrical conductivity. On the other hand it is also possible that the closure of the rift will leave faults or cracks which allow easy penetration of water which enhances conductivity.

The enhanced conductivity along the Tasman Line can also be explained in terms of processes leading to the closure of the rift. During subduction, very old Oceanic crust which is so cold and dense compared with the adjacent continental lithosphere starts to sink into the underlying asthenosphere under its own weight (help by the continual push of newly formed oceanic lithosphere). Subduction zones are ascribed to aqueous fluid, a key factor that enhances electrical conductivity. The descending slab gives rise to dehydration; therefore extra water can be released into the lower crust and mantle by subduction of wet Oceanic crust and Oceanic sediments. The presence of aqueous fluid in the lower crust and mantle enhances deep electrical conductivity. The anomalous conductivity along the Tasman Line is likely due to these processes. Notwithstanding, aqueous fluid is not stable in the lower crust and mantle. Progressive dehydration takes the fluid into the continental crust, that is the dehydrated aqueous fluid flows up to the crust and makes it more electrically conductive than the lower crust and mantle. This explains why the upper crust long the Tasman line is more conductive than the lower crust and mantle.

One can conveniently state that along the Tasman Line, enhanced electrical conductivity in the upper crust is due to both buried sediments and aqueous fluid from progressive dehydration from subduction of wet Oceanic crust while enhanced conductivity in the lower crust and mantle is due to buried conductive sediments and probably due to water from the Earth's surface that penetrates into the interior at subduction zones (Ichiki et al., 2009). Meissner \& Wever (1992) have also demonstrated the existence of meteoric water circulation to great depths in the continental crust and averred that in palaeosuture zones meteoric and metamorphic fluids may be channelled to the lower crust and upper mantle.

In general, the distinct conductivity (sharp contrast in conductivity) constituting the Tasman Line suggests that metasedimentary rocks may have been emplaced in the crust and mantle during the subduction of oceanic crust along the Tasman Line during the process of accretion of present day east Australia. Subduction and collision deform sedimentary sequences between crustal blocks and underthrust them deep into the lower crust or emplace them closer to the surface (Korja, 1997). In addition, Suture zones are characterised by dipping conductive slabs that can extend to the lower crust and beyond (Brown, 1994). Brown (1994) relates the low resistivity of the dipping slabs to black shales which are a major component of flysch sequences in basins between two converging continents. Direen \& Crawford (2003) found it difficult to envisage how a sharp contrast (Tasman Line) in 
the mantle properties can be sustained against thermal erosion over the 500 million years or more since eastern Australia started to be accreted. Since the mantle is solid and if metasidements were buried, which constitute the contrast, it is possible for this contrast to last for hundred million of years.

The enhanced mantle conductivity along the Tasman Line cannot in general be explained in terms of pore fluid alone since pore fluid cannot be retained in the lower crust and mantle for geologically long periods of time and at the same time maintain sufficient pore interconnection that support anomalous conductivity. Free fluid in the lower crust and mantle is lost upwards with time or in thermal or tectonic events, or it could be taken by mineral hydration in retrograde metamorphism associated with cooling (Marquis and Hyndman, 1992). However, water expelled from a descending oceanic slab in a subduction zone can be prevented from reaching the crust and remain conductive in the lower crust if there is the presence of a zone associated with shearing of subcreted sediments which acts as an impermeable cap to fluids. This was inferred by Calvert \& Clowes ( 1990) to explain the source of a lower crustal conductor in the EMSLAB project across the Juan de Fuca subduction zone beneath the west coast of north America. However, in our case, the large conductance (product of conductivity and depth) along the Tasman line suggests that water cannot be the primary source of enhanced conductivity and indicates the presence of an electronic conducting material such as graphite and sulphide bearing metasedimentary rocks.

The magnetic anomaly (figure 3.5) in the vicinity of the Tasman Line can be explained in terms of the presence of magnetic materials. We already mentioned above that one of the laudable explanations for enhanced conductivity along the Tasman Line is the presence of black shales associated with dipping conducting slabs. According to Frost (1991), black shales contain metallic minerals e.g. iron which is present in the form of pyrrhotite $\left(F e S_{2}\right)$ in a sulphur-rich reducing environment of flysch basin as mentioned already. Pyrrhotite has a large susceptibility to provide magnetisations of the order observed in the vicinity of the Tasman Line in Queensland. This gives credit to our explanation to the source of enhanced conductivity along the Tasman Line. The coincident anomalous conductivity and magnetic anomaly measured for both the Carpentaria anomaly and the Tasman Line could also be sourced by a serpentinised arc. Low temperature $\left(<500^{\circ} \mathrm{C}\right)$ hydrothermal alteration of an arc produces serpentine-related minerals such as magnetite and orthopyroxenes (Brown, 1994) which are both conductive and magnetising.

\subsubsection{Eastern Australia}

Eastern Australia is constituted of the Tasman Orogen with Delamerian, Lachlan, Thomson, and New England Orogens as components (figure 3.1). The area of interest covers parts of Thomson, and New England Orogens. For sites in the north, west and east of the Thomson Orogen, we model resistivities between 100 and $300 \Omega \mathrm{m}$ in the upper crust and the resistivities increase with depth to $600 \Omega \mathrm{m}$ in the lower crust and then decrease to resistivities of about $80 \Omega \mathrm{m}$ in the mantle. For sites in the south of the Thomson Orogens, the upper crust is highly conductive with modelled resistivities of less than 10 $\Omega \mathrm{m}$. Resistivities then increase with depth to $80 \Omega \mathrm{m}$ in the Mantle as in other parts of the Orogens. The low crustal resistivity in the south of the Thomson Orogen is due to 
flood water that often settles in this low land and probably due to the extension of the southwest Queensland anomaly to this zone. To the extreme east (New England Orogen), the modelled resistivity is about $800 \Omega \mathrm{m}$ in the crust and this value extends into the upper mantle (see apparent resistivity and phase transfer functions for region 5 , appendix B.4). This is justified by the constant phase of about $45^{\circ}$ for sites in this region (figure 4.5). The relatively high resistivity values are unexpected; we expected the resistivities to decrease from west to east since the age of the orogens decreases from west to east. Some tectonic processes must have caused the relative deformation of the rocks in this region. In addition to this observation, the model shows that conductivity structures in eastern Australia are not uniform. This can be explained in terms of the many faults present in eastern Australia. If eastern Australia was formed by accretional processes beginning at the core of the old cratons to the west at the same time, rocks east of the Tasman Line should have a fairly uniform conductivity.

In general, modelled resistivities in the east of Australia are significantly lower than those measured in the west, especially in the Mt. Isa Block and are many orders higher than those modelled for the Carpentaria anomaly and along the Tasman Line (figures 5.2, 5.14). The difference between resistivities to the west and those to the east of the Tasman Line should be understood since older rocks (with reduced pore fluid; pore fluid decreases with age) in the west are expected to be more resistive than younger Phanerozoic rocks (with greater pore fluid) to the east.

A likely straightforward general interpretation of the data could be as follows: Magnetic activity induces a concentration of current to flow coherently through the Great Artesian Basin. Because of the conductive band like structure (Tasman Line) linking the seas north of Australia in the Gulf of Carpentaria with the southern ocean, currents localize and are channelled into this conductive band. The elevated conductivity along the band like structure extends into the mantle. This is evident from induction vectors and phase split in the vicinity of the Tasman Line. The phase split along the Tasman Line increases with period and has a maximum between 4000 and $8200 \mathrm{~s}$ (figure 4.11). At these periods, there is no strike direction to the west and east of the Tasman Line implying homogeneity. This means that at long periods, the strike direction is recognised along the Tasman Line only. This visible contrast extending to long periods (up to $8200 \mathrm{~s}$ ) certainly extends into the mantle. This is further confirmed by the results of the 3D forward modelling; the $z_{D}$ anomaly extends to at least $80 \mathrm{~km}$ deep into the mantle. The $z_{D}$ anomaly was very instrumental and preludes to our discovery of the extent of the band like conductor into the mantle. It is not possible to attribute the large magnitude of the $z_{D}$ anomaly west of our delineated Tasman Line to anomalous vertical magnetic field fluctuations cause by the Australian coastline since sites to the east and closer to the coastline do not show the same effect. 


\section{Conclusions}

This thesis focuses on Magnetotelluric (MT) and Geomagnetic Depth Sounding (GDS) data analysis and modelling of a considerable part of central and east Australia with the investigation of the Tasman Line as principal objective. It provides new insight on the very important concept of the Tasman Line that holds the key to the understanding of the Evolution of the Australian Plate. It also demonstrates that electromagnetic induction soundings provide us with an elegant way to delineate old crustal boundaries.

The dataset provides a unique opportunity to delineate large scale conductivity structures over an area spanning $670 \mathrm{~km} \times 1615 \mathrm{~km}$. The data were collected during several field campaigns in 2000, 2003 and 2007 and were supplemented with magnetic data from Alice Spring Observatory in order to calculate GDS transfer functions. Since the focus of the study is the resolution of regional structures, the GDS method which identifies dominant regional structures better, compared to MT where electric fields react on small scale deviations from regional structures, proved particularly very useful in resolving lateral conductivity contrast in the heterogeneous conductivity study area.

To recover the strike direction, we preferred the GDS strike which is void of local 3D effects and in addition, we developed a method to explain the effect of current channelling on the the GDS strike direction and concluded that current channelling causes a distortion of the strike direction at the boundary between a conductor and its resistive host. This helped to explain the strike direction at the boundaries of the Tasman Line.

The strength of 3D electromagnetic induction studies is demonstrated in this study and shows that a major basement boundary that was later obscured by sedimentary cover can be mapped. More accurate positioning of major boundaries and structures have been obtained from the method, especially when we combined both MT and GDS. The technique has helped to resolve a portion of the controversial Tasman Line in Queensland and could be used to delineate it further south. The measured and the 3D forward modelled data indubitably define the major electrical conductivity divisions of the surveyed area: (i) the resistive crystalline Mt. Isa Block whose resistivity decreases with depth, (ii) the Eromanga Basin with a thick cover of highly conductive sediments, (iii) a conductive bandlike structure that represents the Carpentaria conductivity anomaly and part of the Tasman Line, (iv) moderate resistive Phanerozioc rocks of eastern Australia compared to more resistive Precambrian (older) rocks to the west. (v) The effect of the coastline in eastern Australia caused by induced electric currents flowing in the conductive Coral Sea and characterised by anomalous Z-fields extends more than $500 \mathrm{~km}$ offshore.

From the model it is found that the rocks of the Mt. Isa Block extend southward under cover than can be discerned from surface Geology. Fraser et al. (1976) \& Gunn et al. (1997) have publised similar results. This is supported by regional gravity and magnetic anomaly data in the region. The uppermost crust of the Mt. Isa Block is very resistive (at 
least $5000 \Omega \mathrm{m}$ ) and thereafter, resistivity decreases with depth; in particular, in the eastern part of Mt. Isa, at depths greater than $10 \mathrm{~km}$, the resistivities resume values modelled for the Carpentaria anomaly. This indicates that at greater depths, the Carpentaria conductivity anomaly extends westward. The regional strike direction is north-south and is very stable along the Carpentaria anomaly and the Tasman Line.

The sediments of the Eromanga Basin are $2500 \pm 500 \mathrm{~m}$ thick and are highly conductive. The modelled resistivities of less than $10 \Omega m$ in the uppermost layer are associated with mineral rich, and a high connected fluid circulation from the many aquifers in the Great Artesian Basin. At short periods, the phase measured in the basin is between $10^{\circ}$ and $15^{\circ}$. This small phase indicates a very thin highly conductive top layer followed by a resistive layer. A striking result from our Electromagnetic induction profiling, indicates structures and compositions through the crust to the upper mantle which are dominant along a single profile from north of site EUL through EUL to KOO. This unique profile stretching up to many hundreds of kilometres in the north-south direction can be interpreted as a plate suture, which was later covered by the sediments of the Artesian Basin. Along the profile, from the electromagnetic induction studies are evidence of a highly conducting material from the crust to the upper mantle. Some structural features seem to be limited to the upper crust. The conductive band like structure has an approximately north-south orientation in northern Queensland and strikes slightly north-southwest as it approaches latitude $25^{\circ} \mathrm{S}$. We conclude that this highly conductive band like structure running northsouth along longitude $(142.5 \pm 0.5)^{\circ} \mathrm{E}$ and extending to at least $80 \mathrm{~km}$ into the mantle is the Tasman Line. Our data is limited to latitude $27^{\circ} \mathrm{S}$ and therefore little can be mentioned about the Tasman Line further south.

The elevated conductivity along the Tasman Line can be explained in terms of collisional processes leading to the closure of the rift (Rodinia break-up boundary). The zone that hosts the Tasman Line can also be described as a metamorphic and or fracture zone; graphitic conductors are often found in such zones (Honkura, 1978). The distinct conductivity (sharp contrast in conductivity) constituting the Tasman Line suggests that metasedimentary rocks may have been emplaced (buried) in the crust and mantle during the subduction of oceanic crust along the Tasman Line in the process of accretion of present day east Australia. In other words, the high conductivity (resistivities less than 20 $\Omega m$ ) along the Tasman Line is mainly due graphite-and sulphide-bearing metasediments and basaltic magmas buried during collisional processes and is supplemented probably by saline fluids transported to the lower crust and mantle via hydro fractures (Watson and Brenan, 1987). Graphite is an important source of conductivity in the form of metasediments trapped in continent-continent palaeosuture zones (Colin, 1994). That means in the case of the Tasman Line, during the process of subduction, where there is addition, from the east, of the Phanerozoic block of the continent through suturing onto the older cratons to the west, the wedge, where the subducting slab has sediments which are formed into geosynclines against the Proterozoic block, has formed a block of highly conducting substances.

From the delineated Taman Line, east of Mt. Isa, we also conclude that the Mt. Isa Belt in Queensland is truncated by a rifted margin formed during the breakup of Rodinia as suggested by Cengage $(2003,2004)$ 
Another prominent feature in the measured data and final model is the Carpentaria conductivity anomaly. It is broad and highly conductive with a north-south strike direction and borders Mt. ISa to the west and extends at least $400 \mathrm{~km}$ east of Mt. Isa. The borders and most of the anomaly is limited to the crust; only the portion that coincides with the Tasman line extends into the mantle. The Carpentaria conductivity anomaly is likely to have been formed by the same tectonic process that produced the Tasman Line since the two coincide in north Queensland.

The measured data and model has also resolved another conductor south of the array with east-northwest strike. This is the southwest Queensland anomaly. This anomaly is connected to the Carpentaria anomaly by the Tasman Line. This is in agreement with previous results published by Woods and Lilly (1979/1980) that the southwest Queensland Anomaly seemed clearly to join the Carpentaria Anomaly. However, its connection with the Arckaringa Anomaly further south is not clear since no sites were installed further south.

Although knowledge of the Australian's plate has been growing at an increasing rate throughout the last four decades, the concept of the Tasman Line has been elusive and a long standing puzzle. Undoubtedly these latest seminal results about the Tasman Line are a milestone in decades long quest by tectonic modellers to uncover it. These influential results may be a key to understanding broad-scale structural differences between eastern and western Australia and will take the debate about the Tasman Line to its most exciting final stage, leading to an increase in the understanding of the tectonic evolution of the Australian plate and hence unravel its complex tectonic history. 


\section{A Telluric and magnetic tensor decomposition}

The equation to be solved is equation (2.59). The matrices (tensors) $\underline{\underline{D}}$ and $\underline{\underline{C}}$ each have four real elements, and $\underline{\underline{Z}}_{R}$ four complex elements. This gives a total of 16 real unknowns. If we write matrix equation (2.59) out separately for each of its four element quations lead to 8 real equations for real and imaginary parts separately. This is an underdetermined problem since we have 16 unknows. Therefore some assumptions have to be made. It is assumed that the regional structure is 2D (Smith, 1997), so that in coordinate systems aligned with the regional strike $\underline{Z}_{R}$ has zero diagonal elements. This eliminates four unknow. Smith (1997) simplied the distortion matrices in equation (2.59) to the take the form:

telluric

$$
D=\left(\begin{array}{ll}
1 & c \\
b & 1
\end{array}\right)
$$

where $\mathrm{b}$ and $\mathrm{c}$ are real and frequency-independent. The diagonal elements of the telluric matrix are set to one because they are absorbed in the regional impendance, corresponding to the known unresolved problem of gain + anisotropy effects (Groom and Bailey, 1989b). This reduces two more unknows.

\section{magnetic}

$$
C=\left(\begin{array}{ll}
\gamma & 0 \\
0 & \epsilon
\end{array}\right)
$$

where $\gamma$ and $\epsilon$ are real and frequency-independent. The reduction of the off-diagonal elements to zero is due to the indetermination in solving them. This means that the anomalous magnetic fields aligned with the induced regional magnetic field are absorbed in it. Given the indeterminacy of the parallel distorted field, the off-diagonal elements are assumed to vanish(Smith 1997). This eliminates two more unknowns.

After all the assumptions, equation (2.59) now has eight real unknowns; b, c, $\gamma, \epsilon$ and the real and imaginary parts of $Z_{y x}^{R}$ and $Z_{x y}^{R}$ corresponding to eight degrees of freedom. The unknow regional impedances, $Z_{y x}^{R}$ and $Z_{x y}^{R}$, can be solved in terms of the measured impedance $Z_{x x}, Z_{x y}, Z_{y x}$ and $Z_{y y}$.

With the assumption of a $2 \mathrm{D}$ regional impedance $Z_{R}$, in the strike coordinate and using the simplied distortion matrices, equation (2.59) can be expanded to give:

$$
\left(\begin{array}{cc}
Z_{x x} & Z_{x y} \\
Z_{y x} & Z_{x x}
\end{array}\right)=\left(\begin{array}{cc}
c Z_{y x}^{R}-\epsilon Z_{x y} Z_{y x}^{R} & Z_{x y}^{R}-\gamma Z_{x x} Z_{x y}^{R} \\
Z_{y x}^{R}-\epsilon Z_{y y} Z_{y x}^{R} & b Z_{x y}^{R}-\gamma Z_{y x} Z_{x y}^{R}
\end{array}\right)
$$


Writing this out separately for each of its four elements and expressing each of the unknown regional impedances in terms of the measured impedance b, $\mathrm{c}, \gamma$ and $\epsilon$ yields

$$
\begin{aligned}
Z_{y x}^{R} & =Z_{x x} /\left(c-\epsilon Z_{x y}\right) \\
Z_{x y}^{R} & =Z_{y x} /\left(1-\epsilon Z_{y y}\right) \\
Z_{x y}^{R} & =Z_{x y} /\left(1-\gamma Z_{x x}\right) \\
Z_{x y}^{R} & =Z_{y y} /\left(b-\gamma Z_{y x}\right)
\end{aligned}
$$

The unknown regional impedances $Z_{y x}^{R}$ and $Z_{y x}^{R}$, can be eliminated, to yield two complex linear equations for the real unknowns b, c, $\gamma$ and $\epsilon$ :

$$
\begin{aligned}
& Z_{x x}=c Z_{y x}+\epsilon\left(Z_{x x} Z_{y y}-Z_{y x} Z_{x y}\right) \\
& Z_{y y}=b Z_{x y}+\gamma\left(Z_{x x} Z_{y y}-Z_{y x} Z_{x y}\right)
\end{aligned}
$$

If we take real and imaginary parts separately, we arrive at the solution of the parameters $\mathrm{b}, \mathrm{c}, \gamma$ and $\epsilon$ in terms of the measured impedance:

$$
\begin{aligned}
\gamma & =\frac{\operatorname{Re} Z_{x y} I m Z_{y y}-\operatorname{Im} Z_{x y} \operatorname{Re} Z_{y y}}{\operatorname{Re} Z_{x y} \operatorname{Im}(\operatorname{det} Z)-\operatorname{Im} Z_{x y} \operatorname{Re}(\operatorname{det} Z)} \\
\epsilon & =\frac{\operatorname{Re} Z_{y x} \operatorname{Im} Z_{x x}-\operatorname{Im} Z_{y x} \operatorname{Re} Z_{x x}}{\operatorname{Re} Z_{y x} \operatorname{Im}(\operatorname{det} Z)-\operatorname{Im} Z_{y x} \operatorname{Re}(\operatorname{det} Z)} \\
b & =\frac{\operatorname{Re} Z_{y y} \operatorname{Im}(\operatorname{det} Z)-\operatorname{Im} Z_{y y} \operatorname{Re}(\operatorname{det} Z)}{\operatorname{Re} Z_{x y} \operatorname{Im}(\operatorname{det} Z)-\operatorname{Im} Z_{x y} \operatorname{Re}(\operatorname{det} Z)} \\
c & =\frac{\operatorname{Re} Z_{x x} \operatorname{Im}(\operatorname{det} Z)-\operatorname{Im} Z_{x x} \operatorname{Re}(\operatorname{det} Z)}{\operatorname{Re} Z_{y x} \operatorname{Im}(\operatorname{det} Z)-\operatorname{Im} Z_{y x} \operatorname{Re}(\operatorname{det} Z)}
\end{aligned}
$$

The regional impedances $Z_{x y}^{R}$ and $Z_{y x}^{R}$ can now be evaluted as function of measured impedance Z:

$$
\begin{aligned}
& Z_{x y}^{R}=Z_{y x} /\left(1-\epsilon Z_{y y}\right) \\
& Z_{x y}^{R}=Z_{x y} /\left(1-\gamma Z_{x x}\right)
\end{aligned}
$$




\section{B Field sites and data}

\section{B.1 Names and locations of MT sites}

\begin{tabular}{l|c|c}
\multicolumn{3}{c}{2000 sites } \\
station name & longitude & latitude \\
WRA & 134.325 & -19.925 \\
DOR & 131.500 & -22.167 \\
ASP & 133.880 & -23.761 \\
ULU & 131.858 & -25.400 \\
VIC & 132.925 & -25.983 \\
ANO & 134.900 & -25.683
\end{tabular}

Table B.1: Station names and coordinates of the 2000 MT sites.

\begin{tabular}{l|c|c||l|l|r}
\multicolumn{6}{|c}{2003 sites } \\
station name & longitude & latitude & station name & longitude & latitude \\
ARG & 136.590 & -21.650 & BRE* $^{*}$ & 139.640 & -23.703 \\
SOU & 136.990 & -19.920 & MTE* & 142.431 & -21.720 \\
HEA & 138.400 & -21.230 & NAS* & 137.110 & -20.979 \\
KAJ & 139.860 & -19.950 & & & \\
ALD & 139.920 & -22.430 & & & \\
KOO & 139.760 & -24.990 & & & \\
EUL & 141.460 & -21.270 & & \\
DIA & 141.470 & -23.530 & & \\
WIN & 143.430 & -22.290 & & & \\
NUM & 135.074 & -23.954 & & & \\
RID & 134.132 & -22.960 & & &
\end{tabular}

Table B.2: Station names and coordinates of the 2003 MT sites. Asterisks indicate sites with less than 5 days of data.

Sites with very little measurement time came as a result of difficulties encountered during the campaign. Either due to lack of enough intruments or due to other problems encountered on the field. For example, in the 2003 Campaign some sites run only at night and for some sites that were expected to run through out the campaign, there were problems such as dried out electrodes. This led to either loss of electric fields at some sites, or loss of magnetic and electric components at some sites. For the 2007 campaign, the site COOL lost it magnetic component after about 25 days of measurement, to make full use of its 


\begin{tabular}{l|c|c||l|l|l} 
2tation name & longitude & latitude & station name & longitude & latitude \\
WIND & 143.052 & -25.561 & INJU & 148.711 & -25.841 \\
LAGL & 146.721 & -22.485 & CARE* & 144.286 & -21.407 \\
STON* & 143.245 & -24.357 & HOME* & 145.690 & -20.400 \\
FORE* & 147.767 & -25.884 & CAPE & 148.124 & -23.123 \\
PLAI & 146.671 & -21.483 & ISIS & 144.341 & -24.211 \\
TORE* & 145.029 & -20.823 & TAMB & 146.403 & -24.910 \\
LIST* $^{\text {PEAK }}$ & 145.208 & -25.235 & ARAM & 145.311 & -22.950 \\
CARN* & 147.743 & -22.608 & HUGH & 144.228 & -20.964 \\
MUTT* & 148.363 & -24.938 & BELC* & 146.837 & -21.513 \\
BARC* & 144.506 & -22.498 & ALPH & 146.654 & -23.607 \\
AUGA & 145.264 & -23.607 & COOL & 145.470 & -26.631 \\
& 146.618 & -25.788 & & &
\end{tabular}

Table B.3: Station names and coordinates of the 2007 MT sites. Asterisks indicate sites with less than 5 days of data and whose data have not contributed much in our analysis.

data and include long periods, we substituded it magnetic field with that of a neighbouring site (Tamb) and then corrected for the effect of anomalos magnetic fieds between them.

Sites that lost electric fieds completely due to drift in both components and whose data could not be analysed have not been included in the tables above.

\section{B.2 Processed MT data}

Figures B.1 to B. 4 show plots of complete MT transfer functions (apparent resistivities and phase values) of the off-diagonal impedance tensor elements for the 5 regions discussed in chapter 4 (figures 4.5-4.8). The plots are in an unrotated ( North-South/East-West) Coordinate system. The period range for each site is a function of the operating time at the site. 

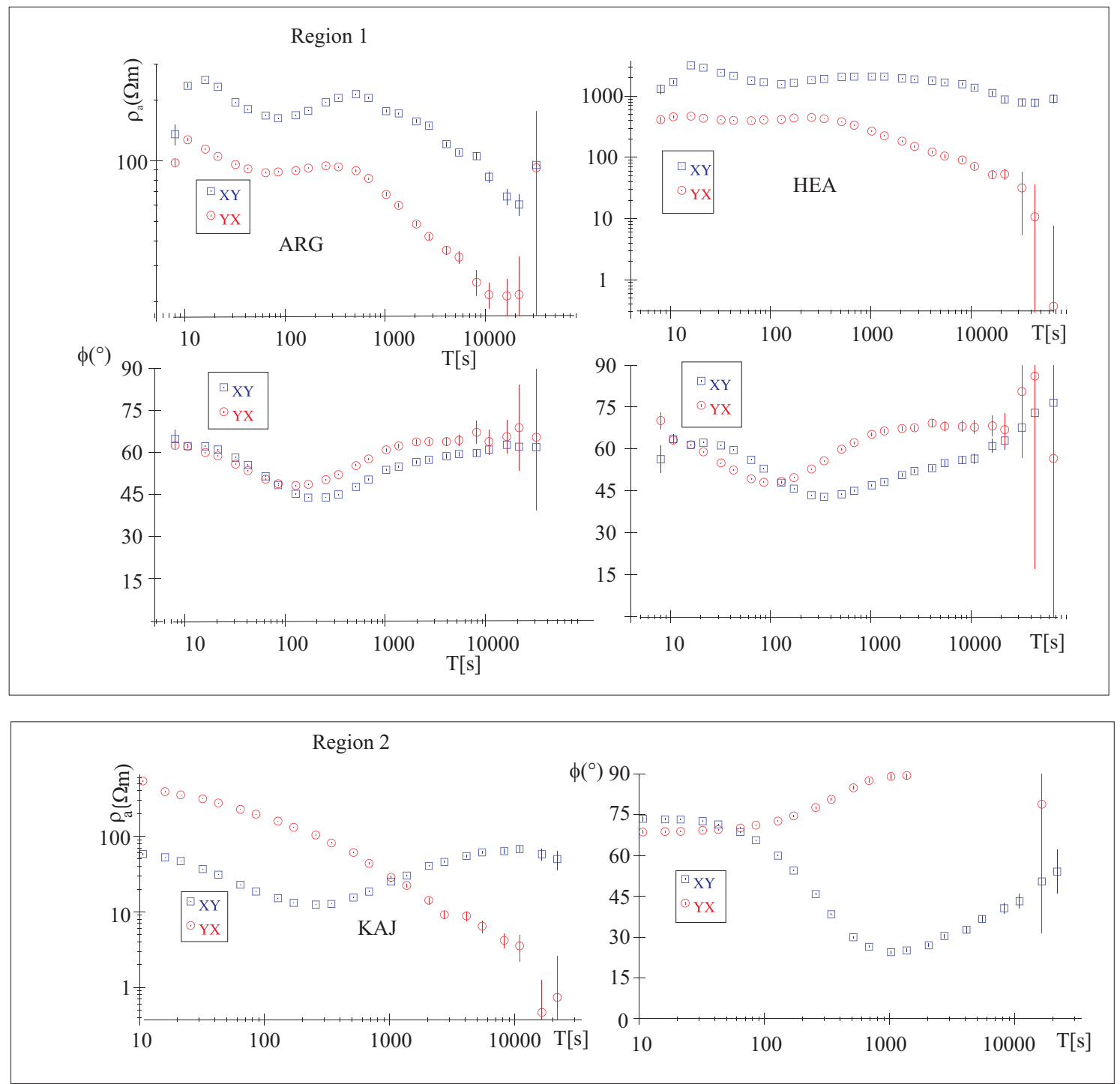

Figure B.1: MT transfer functions in an unrotated coordinate frame in regions 1 and 2. xy (E-polarisation) is north-south and $\mathrm{yx}(\mathrm{B}$-polarisation) is east-west. 

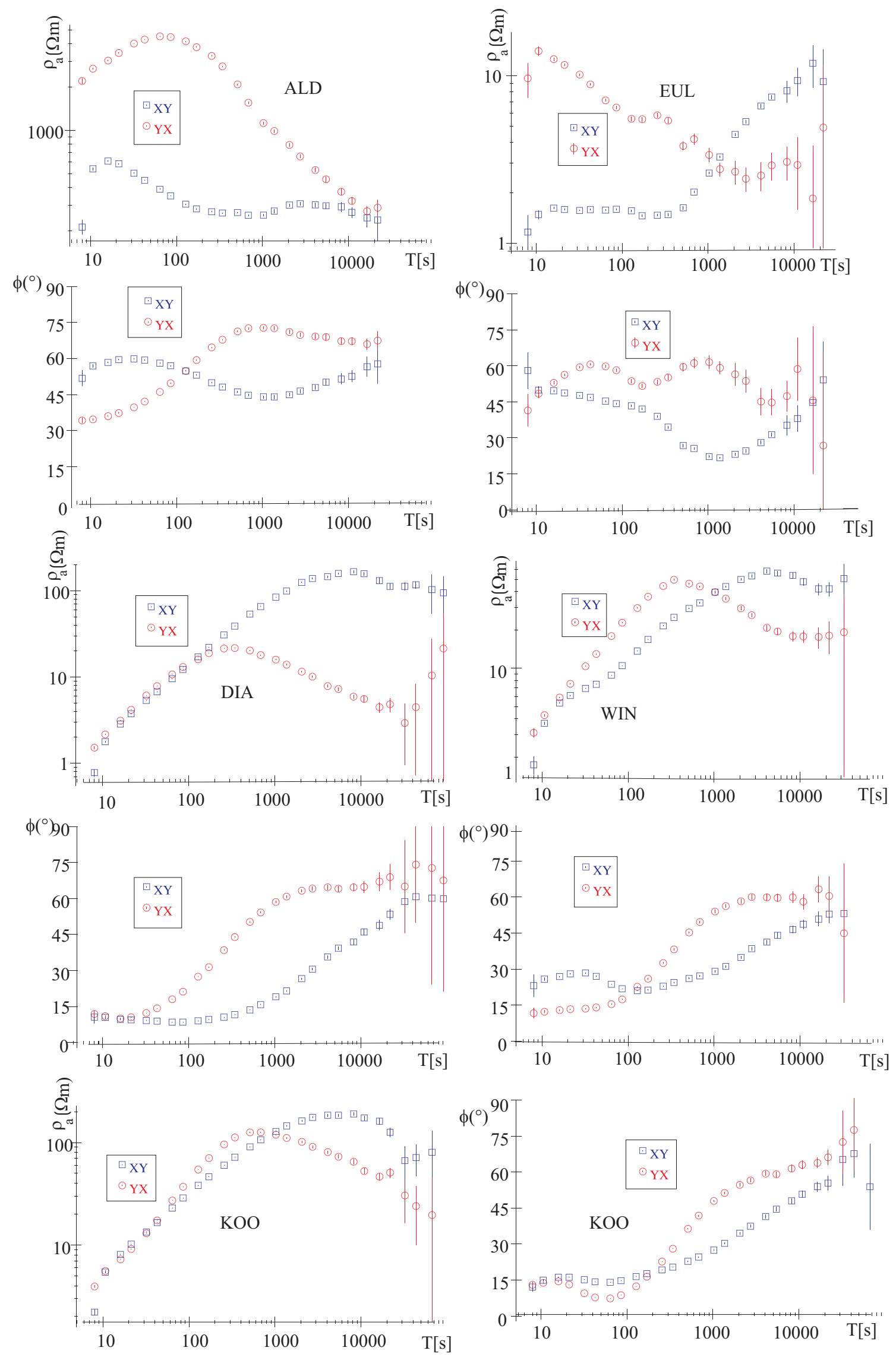

Figure B.2: Unrotated MT transfer functions; representative of region 3. 

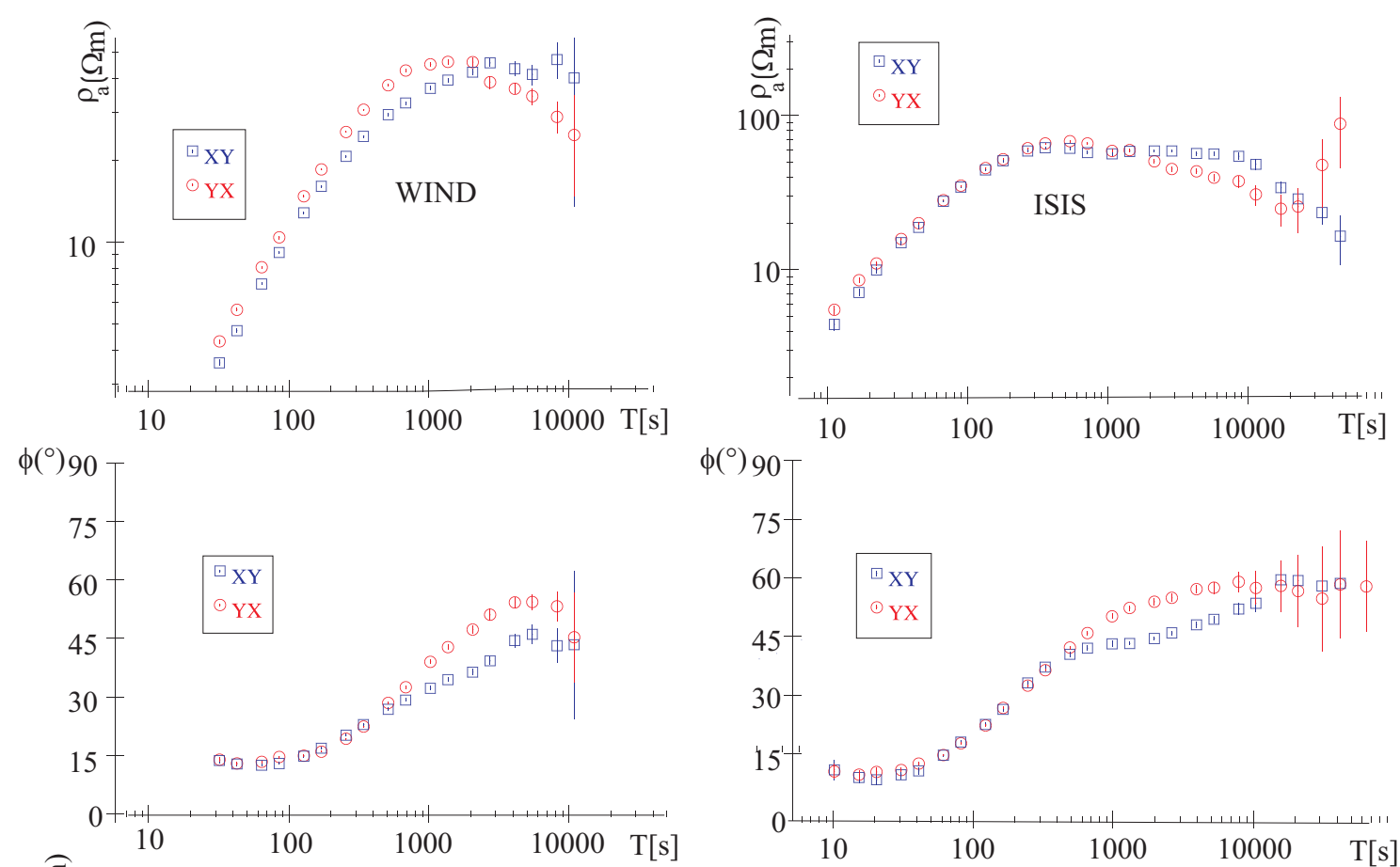

$\phi\left(^{\circ}\right) 90 \mathrm{~T}$
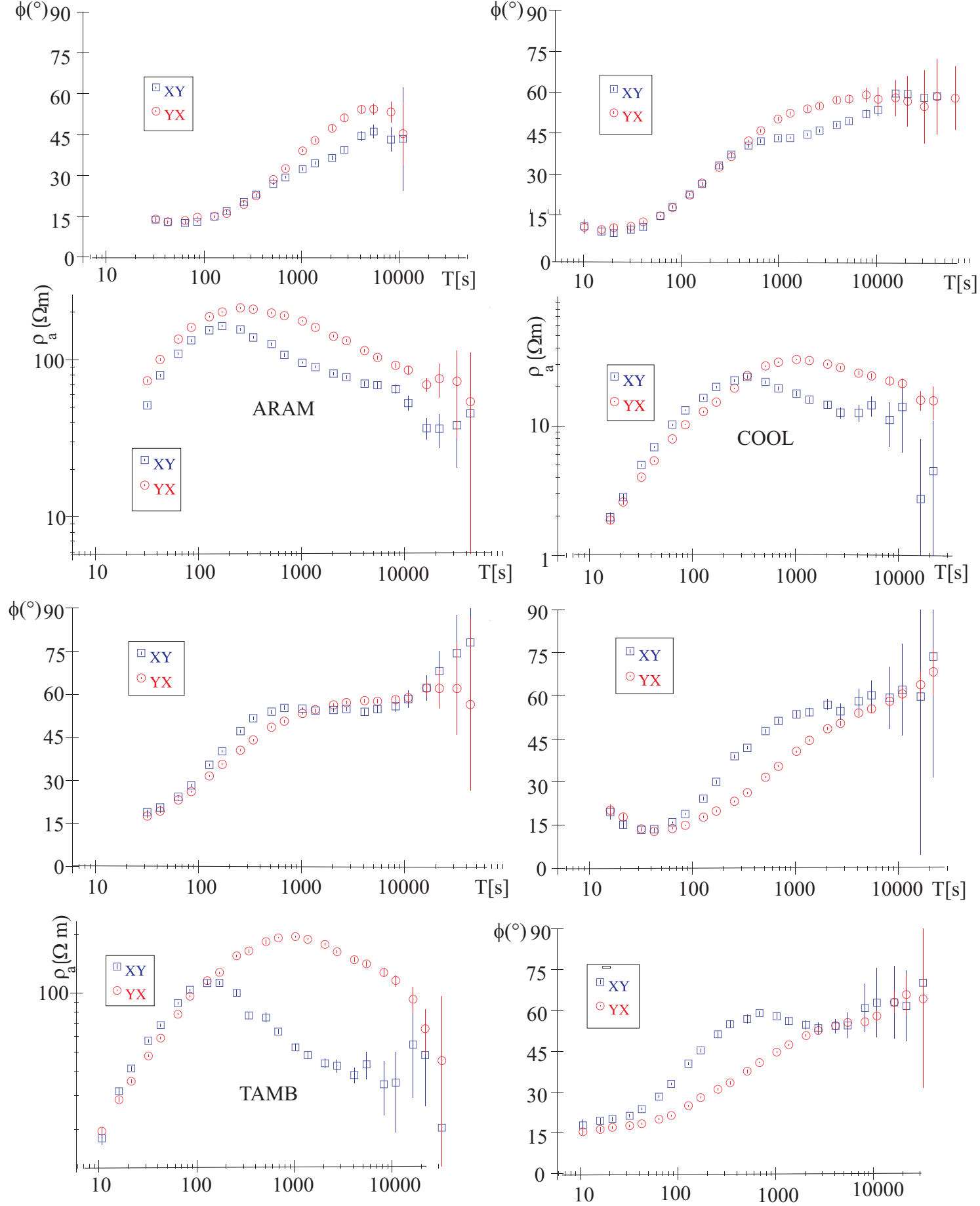

Figure B.3: Unrotated MT transfer functions; representative of region 4. 

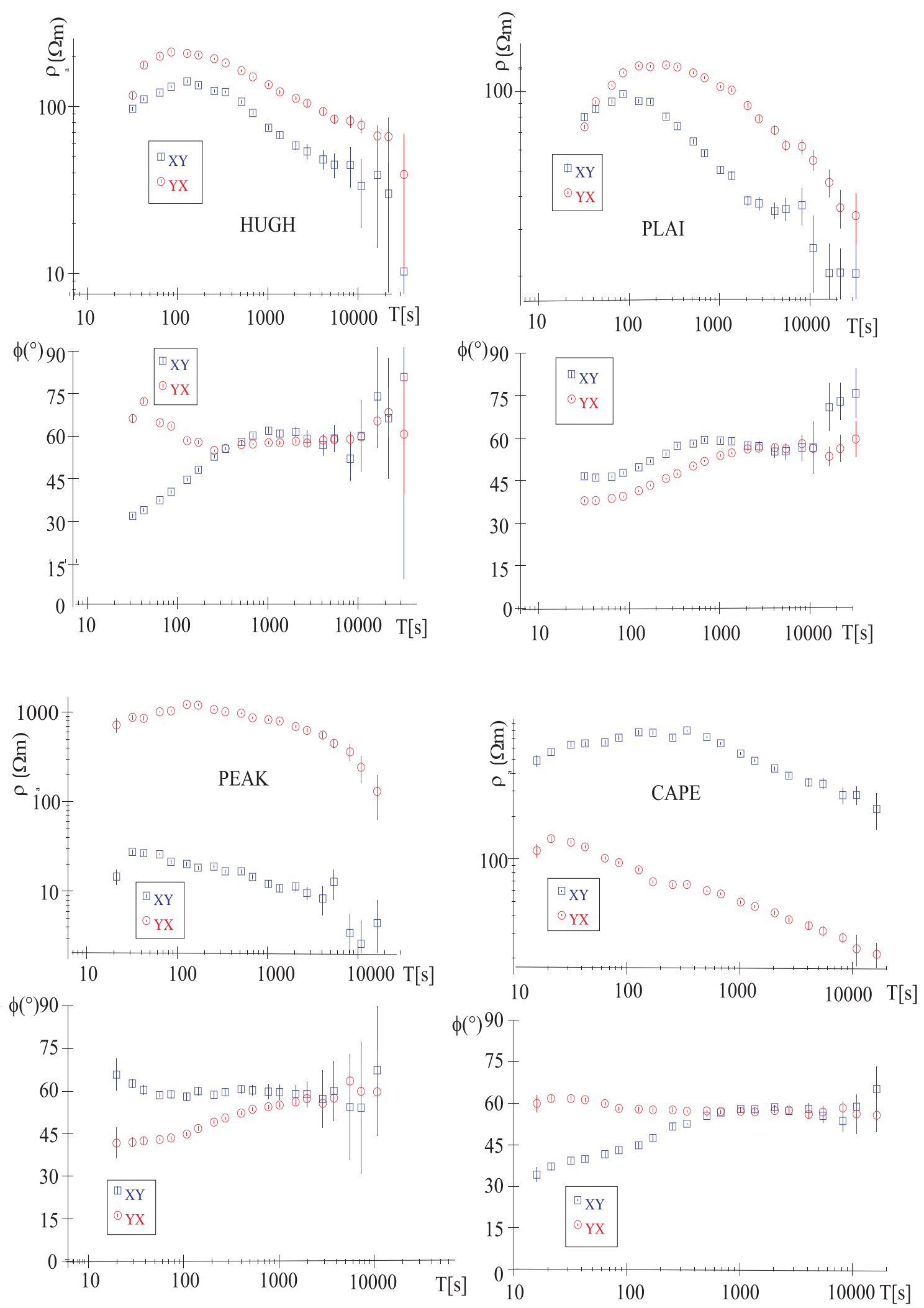

Figure B.4: Unrotated MT transfer functions; representative of region 5. 
Figures B.5 and B.6 show plots of apparent resistivities and phase values of the off-diagonal impedance tensor elements for sites not included in figures 4.5 to 4.8 or B.1 to B.4. The plots are in an unrotated ( North-South/East-West) Coordinate system. The period range for each site is a function of the operating time at the site. 

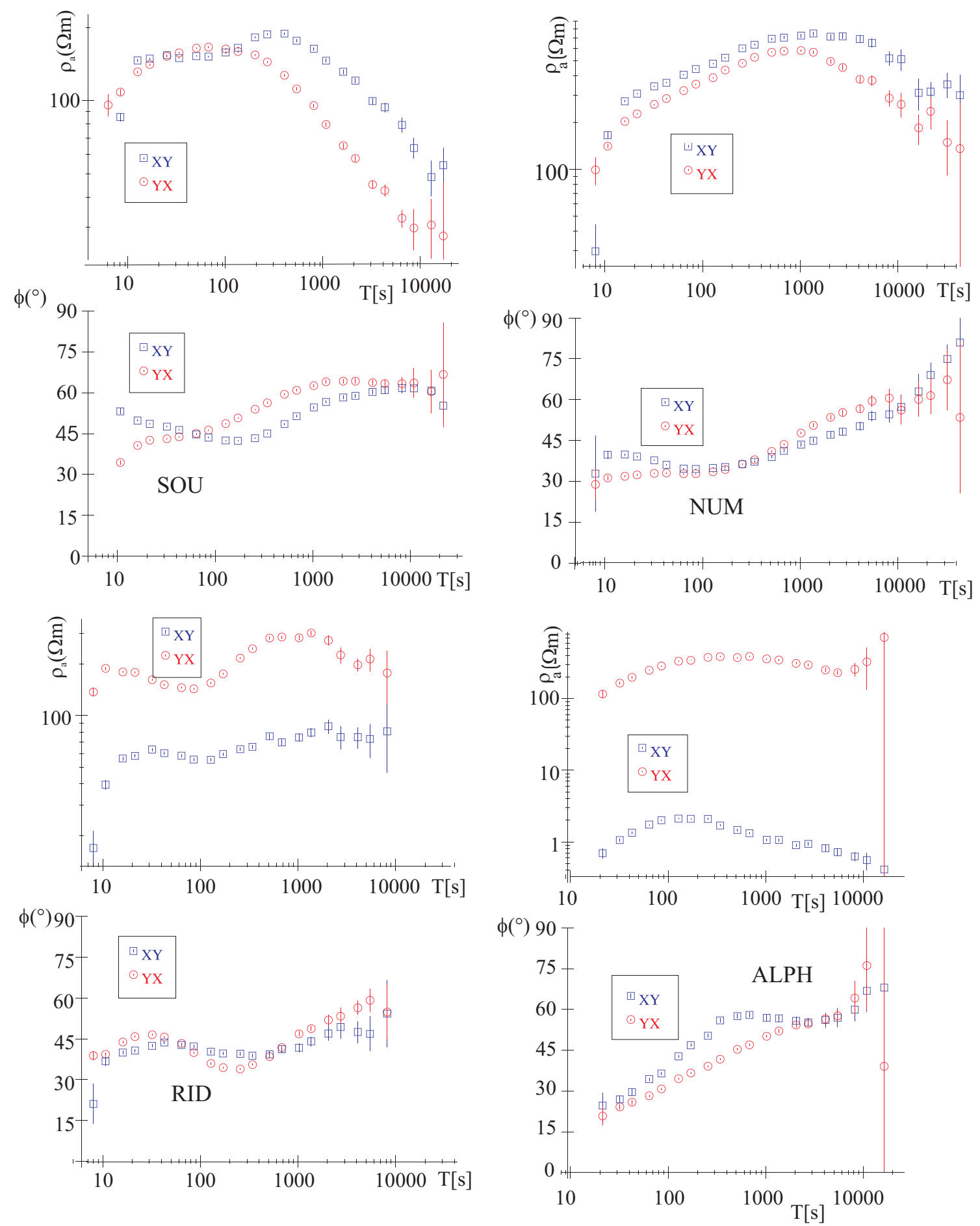

Figure B.5: Unrotated MT-transfer functions showing apparent resistivity and phase curves for other MT sites of this study. 

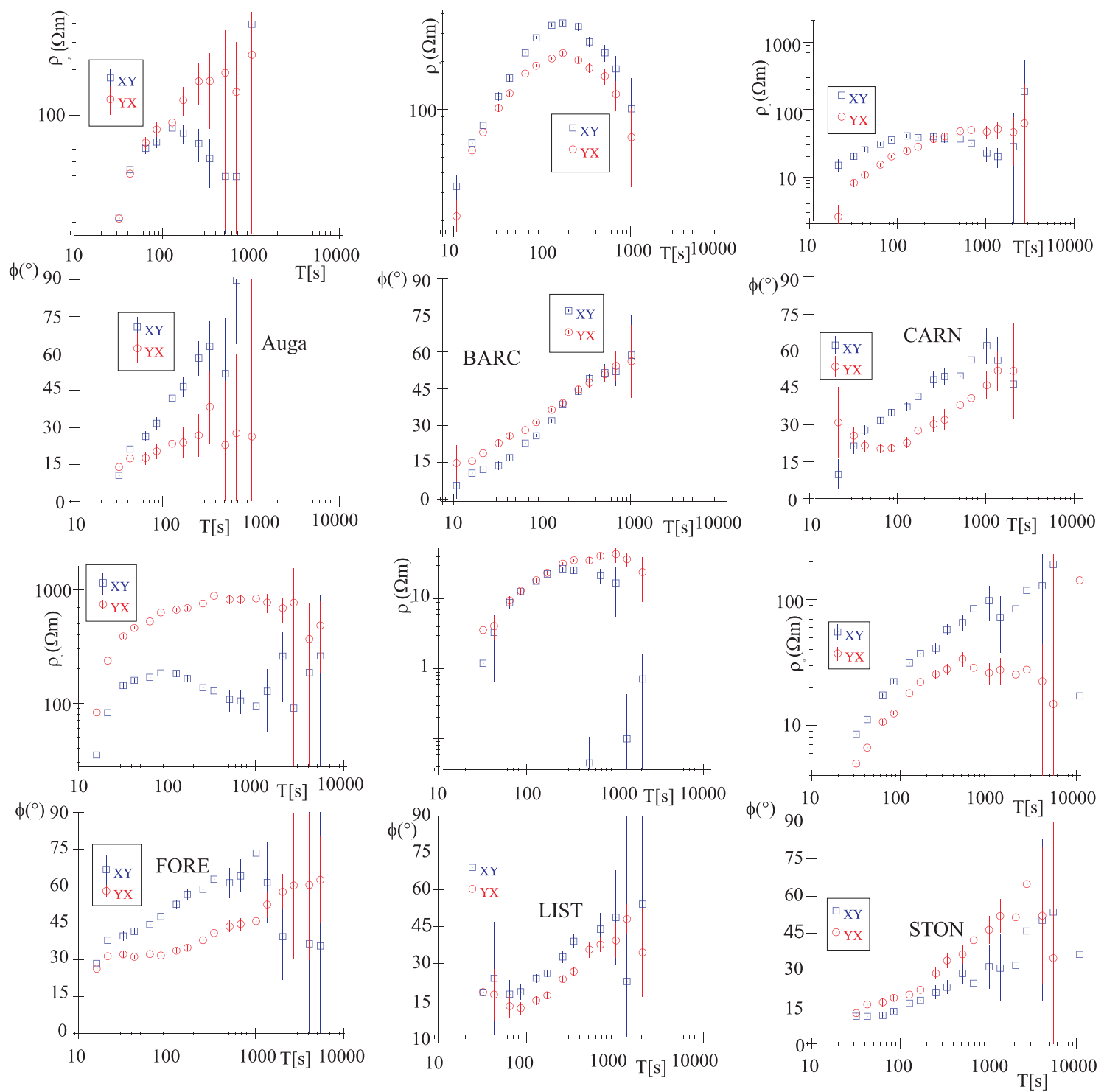

Figure B.6: Unrotated MT-transfer functions showing apparent resistivity and phase curves for other MT sites of this study; these are mostly the short period sites whose results have not contributed much in our analysis. 


\section{Explicit expressions for transfer functions}

\section{C.1 Magnetotellurics}

The elements of the impedance tensor are explicitly expressed as follows:

$$
\begin{aligned}
& Z_{x x}=\left(\left\langle E_{x} B_{x}^{*}\right\rangle\left\langle B_{y} B_{y}^{*}\right\rangle-\left\langle E_{x} B_{y}^{*}\right\rangle\left\langle B_{y} B_{x}^{*}\right\rangle\right) / D \\
& Z_{x y}=\left(\left\langle E_{x} B_{y}^{*}\right\rangle\left\langle B_{x} B_{x}^{*}\right\rangle-\left\langle E_{x} B_{x}^{*}\right\rangle\left\langle B_{x} B_{y}^{*}\right\rangle\right) / D \\
& Z_{y x}=\left(\left\langle E_{y} B_{x}^{*}\right\rangle\left\langle B_{y} B_{y}^{*}\right\rangle-\left\langle E_{y} B_{y}^{*}\right\rangle\left\langle B_{y} B_{x}^{*}\right\rangle\right) / D \\
& Z_{y y}=\left(\left\langle E_{y} B_{y}^{*}\right\rangle\left\langle B_{x} B_{x}^{*}\right\rangle-\left\langle E_{y} B_{x}^{*}\right\rangle\left\langle B_{x} B_{y}^{*}\right\rangle\right) / D \\
& Z_{z x}=\left(\left\langle B_{z} B_{x}^{*}\right\rangle\left\langle B_{y} B_{y}^{*}\right\rangle-\left\langle B_{Z} B_{y}^{*}\right\rangle\left\langle B_{y} B_{x}^{*}\right\rangle\right) / D \\
& Z_{z y}=\left(\left\langle B_{z} B_{y}^{*}\right\rangle\left\langle B_{x} B_{x}^{*}\right\rangle-\left\langle B_{z} B_{x}^{*}\right\rangle\left\langle B_{x} B_{y}^{*}\right\rangle\right) / D .
\end{aligned}
$$

The errors of the MT transfer fuunctions, according to Watermann (1984) are given by the following expressions:

$$
\begin{aligned}
\Delta Z_{x x} & =\sqrt{f_{2}(\nu)\left\langle E_{x} E_{x}^{*}\right\rangle\left(1-R_{x}\right) /\left(\left\langle B_{x} B_{x}^{*}\right\rangle(1-U)\right)} \\
\Delta Z_{x y} & =\sqrt{f_{2}(\nu)\left\langle E_{x} E_{x}^{*}\right\rangle\left(1-R_{x}\right) /\left(\left\langle B_{y} B_{y}^{*}\right\rangle(1-U)\right)} \\
\Delta Z_{y x} & =\sqrt{f_{2}(\nu)\left\langle E_{y} E_{y}^{*}\right\rangle\left(1-R_{y}\right) /\left(\left\langle B_{x} B_{x}^{*}\right\rangle(1-U)\right)} \\
\Delta Z_{y y} & =\sqrt{f_{2}(\nu)\left\langle E_{y} E_{y}^{*}\right\rangle\left(1-R_{y}\right) /\left(\left\langle B_{y} B_{y}^{*}\right\rangle(1-U)\right)} \\
\Delta Z_{z x} & =\sqrt{f_{2}(\nu)\left\langle B_{z} B_{z}^{*}\right\rangle\left(1-R_{z}\right) /\left(\left\langle B_{x} B_{x}^{*}\right\rangle(1-U)\right)} \\
\Delta Z_{z y} & =\sqrt{f_{2}(\nu)\left\langle B_{z} B_{z}^{*}\right\rangle\left(1-R_{z}\right) /\left(\left\langle B_{y} B_{y}^{*}\right\rangle(1-U)\right)}
\end{aligned}
$$

where

$$
D=\left(\left\langle B_{x} B_{x}^{*}\right\rangle\left\langle B_{y} B_{y}^{*}\right\rangle-\left\langle B_{x} B_{y}^{*}\right\rangle\left\langle B_{y} B_{x}^{*}\right\rangle\right) .
$$

$R_{i}$ denote quadratic bivariate cohence and $\mathrm{U}$ the quadratic univariate coherence: 


$$
\begin{aligned}
R_{x} & =\left(Z_{x x}\left\langle B_{x} E_{x}^{*}\right\rangle+Z_{x y}\left\langle B_{y} E_{x}^{*}\right\rangle\right) /\left\langle E_{x} E_{x}^{*}\right\rangle \\
R_{Y} & =\left(Z_{y x}\left\langle B_{x} E_{y}^{*}\right\rangle+Z_{y y}\left\langle B_{y} E_{y}^{*}\right\rangle\right) /\left\langle E_{y} E_{y}^{*}\right\rangle \\
R_{z} & =\left(Z_{z x}\left\langle B_{x} B_{z}^{*}\right\rangle+Z_{z y}\left\langle B_{y} B_{z}^{*}\right\rangle\right) /\left\langle B_{z} B_{z}^{*}\right\rangle \\
U & =\left\langle B_{x} B_{y}^{*}\right\rangle\left\langle B_{y} B_{x}^{*}\right\rangle /\left\langle B_{x} B_{x}^{*}\right\rangle\left\langle B_{y} B_{y}^{*}\right\rangle
\end{aligned}
$$

Moreover

$f_{2}(\nu)=\frac{4}{4-\nu} F_{4, \nu-4}(\beta)$

where $F_{4, \nu-4}(\beta)$ is the Fisher distribution with $\beta=68 \% . \nu$ is the number of degrees of freedom.

\section{C.2 Geomagnetic Depth Sounding-GDS}

The elements of the perturbation tensor are expressed as follows:

$$
\begin{aligned}
h H & =\left(\left\langle B_{x} B_{x}^{n *}\right\rangle\left\langle B_{y}^{n} B_{y}^{*}\right\rangle-\left\langle B_{x} B_{y}^{n *}\right\rangle\left\langle B_{y}^{n} B_{x}^{*}\right\rangle\right) / D \\
h D & =\left(\left\langle B_{x} B_{y}^{n *}\right\rangle\left\langle B_{x}^{n} B_{x}^{*}\right\rangle-\left\langle B_{x} B_{x}^{n *}\right\rangle\left\langle B_{x}^{n} B_{y}^{*}\right\rangle\right) / D \\
d H & =\left(\left\langle B_{y} B_{x}^{n *}\right\rangle\left\langle B_{y}^{n} B_{y}^{*}\right\rangle-\left\langle B_{y} B_{y}^{n *}\right\rangle\left\langle B_{y}^{n} B_{x}^{*}\right\rangle\right) / D \\
d D & =\left(\left\langle B_{y} B_{y}^{n *}\right\rangle\left\langle B_{x}^{n} B_{x}^{*}\right\rangle-\left\langle B_{y} B_{x}^{n *}\right\rangle\left\langle B_{x}^{n} B_{y}^{*}\right\rangle\right) / D \\
z H & =\left(\left\langle B_{z} B_{x}^{n *}\right\rangle\left\langle B_{y}^{n} B_{y}^{*}\right\rangle-\left\langle B_{z} B_{y}^{n *}\right\rangle\left\langle B_{y}^{n} B_{x}^{*}\right\rangle\right) / D \\
z D & =\left(\left\langle B_{z} B_{y}^{n *}\right\rangle\left\langle B_{x}^{n} B_{x}^{*}\right\rangle-\left\langle B_{z} B_{x}^{n *}\right\rangle\left\langle B_{x}^{n} B_{y}^{*}\right\rangle\right) / D
\end{aligned}
$$

$$
\begin{aligned}
\Delta h H & =\sqrt{f_{2}(\nu)\left\langle B_{x} B_{x}^{*}\right\rangle\left(1-R_{x}\right) /\left(\left\langle B_{x}^{n} B_{x}^{*}\right\rangle(1-U)\right)} \\
\Delta h D & =\sqrt{f_{2}(\nu)\left\langle B_{x} B_{x}^{*}\right\rangle\left(1-R_{x}\right) /\left(\left\langle B_{y}^{n} B_{y}^{*}\right\rangle(1-U)\right)} \\
\Delta d H & =\sqrt{f_{2}(\nu)\left\langle B_{y} B_{y}^{*}\right\rangle\left(1-R_{y}\right) /\left(\left\langle B_{x}^{n} B_{x}^{*}\right\rangle(1-U)\right)} \\
\Delta d D & =\sqrt{f_{2}(\nu)\left\langle B_{y} B_{y}^{*}\right\rangle\left(1-R_{y}\right) /\left(\left\langle B_{y}^{n} B_{y}^{*}\right\rangle(1-U)\right)} \\
\Delta z H & =\sqrt{f_{2}(\nu)\left\langle B_{z} B_{z}^{*}\right\rangle\left(1-R_{z}\right) /\left(\left\langle B_{x}^{n} B_{x}^{*}\right\rangle(1-U)\right)} \\
\Delta z D & =\sqrt{f_{2}(\nu)\left\langle B_{z} B_{z}^{*}\right\rangle\left(1-R_{z}\right) /\left(\left\langle B_{y}^{n} B_{y}^{*}\right\rangle(1-U)\right)}
\end{aligned}
$$


where

$$
\begin{aligned}
D & =\left(\left\langle B_{x}^{n} B_{x}^{n *}\right\rangle\left\langle B_{y}^{n} B_{y}^{n *}\right\rangle-\left\langle B_{x}^{n} B_{y}^{n *}\right\rangle\left\langle B_{y}^{n} B_{x}^{n *}\right\rangle\right) \\
R_{x} & =\left(h H\left\langle B_{x}^{n} B_{x}^{*}\right\rangle+h D\left\langle B_{y}^{n} B_{x}^{*}\right\rangle\right) /\left\langle B_{x} B_{x}^{*}\right\rangle \\
R_{Y} & =\left(d H\left\langle B_{x}^{n} B_{y}^{*}\right\rangle+d D\left\langle B_{y}^{n} B_{y}^{*}\right\rangle\right) /\left\langle B_{y} B_{y}^{*}\right\rangle \\
R_{z} & =\left(z H\left\langle B_{x}^{n} B_{z}^{*}\right\rangle+z D\left\langle B_{y}^{n} B_{z}^{*}\right\rangle\right) /\left\langle B_{z} B_{z}^{*}\right\rangle \\
U & =\left\langle B_{x}^{n} B_{y}^{n *}\right\rangle\left\langle B_{y}^{n} B_{x}^{n *}\right\rangle /\left\langle B_{x}^{n} B_{x}^{n *}\right\rangle\left\langle B_{y}^{n} B_{y}^{n *}\right\rangle .
\end{aligned}
$$

\section{C.3 Göttinger Data Format}

\section{C.3.1 *.mtt-Format}

The MT transfer functions at each measurement site are given by the following linear expressions:

$$
\begin{aligned}
& E_{x}=Z_{x x} B_{x}+Z_{x y} B_{y} \\
& E_{y}=Z_{y x} B_{x}+Z_{y y} B_{y} \\
& B_{z}=Z_{z x} B_{x}+Z_{z y} B_{y}
\end{aligned}
$$

which are saved in ASCII-data in the following format:

\begin{tabular}{lccccccc} 
Frequency & \multicolumn{7}{c}{ Degrees of freedom } \\
$\operatorname{Re} Z_{x x}$ & $\operatorname{Im} Z_{x x}$ & $\operatorname{Re} Z_{x y}$ & $\operatorname{Im} Z_{x y}$ & $\operatorname{Re} Z_{y x}$ & $\operatorname{Im} Z_{y x}$ & $\operatorname{Re} Z_{y y}$ & $\operatorname{Im} Z_{y y}$ \\
$\Delta Z_{x x}$ & $\Delta Z_{x y}$ & $\Delta Z_{y x}$ & $\Delta Z_{y y}$ & $\operatorname{Re} Z_{z x}$ & $\operatorname{Im} Z_{z x}$ & $\operatorname{Re} Z_{z y}$ & $\operatorname{Im} Z_{z y}$ \\
$\Delta Z_{z x}$ & $\Delta Z_{z y}$ & $R_{x}$ & $R_{y}$ & $R_{z}$ & & &
\end{tabular}

The frequency has its usual units of $\mathrm{Hz}$ and the elements of the impedance tensor have as units $\mathrm{Km} / \mathrm{s}$.

\section{C.3.2*.gdt-Format}

If we expand equation 2.62, we obtain the following linear relations between the amalous field at the observation station and the normal field at the reference station:

$$
\begin{aligned}
B_{x}^{a} & =h_{H} B_{x}^{n}+h_{D} B_{y}^{n} \\
B_{y}^{a} & =d_{H} B_{x}^{n}+d_{D} B_{y}^{n} \\
B_{z}^{a} & =z_{H} B_{x}^{n}+z_{D} B_{y}^{n} .
\end{aligned}
$$

The format is built in a similar manner as the ${ }^{*}$.mtt-format. The magnetic transfer functions are dimensionless. 


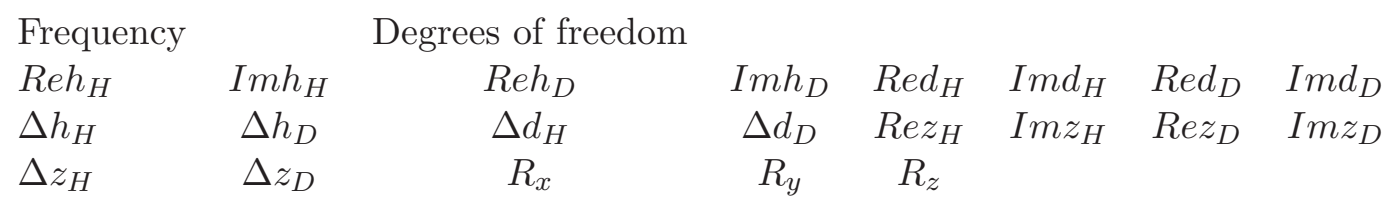




\section{Programs used}

This chapter gives a summary of the programs used to analyse the data in this thesis. To begin, the time series data from the RAP-Loggers saved in the binary .goe format (section 5.1) are read using the following programs:

ramtra[Steveling]

congoe[Steveling].

For the processing of the time series and determination of the transfer functions, the following programs were used:

select[GU, Bahr]

dezidezi[Eisel, Bahr, Steveling]

analys[Bahr]

\section{D.1 Programs to analyse and display data}

\section{D.1.1 emdisp}

The program emdisp (Leibecker, 2000; Gatzemeier, 2002 \& Moorkamp, 2003) reads Göttigen type MT and GDS data (.mtt/.gdt format, (appendix C)) and produces files for map plots of certain MT or GDS parameters that can be plotted with the GMT mapping tool (Wessel \& Smith, 1998). The following calculation are possible from the current version of the program:

\section{Magnetotellurics}

Apparent resistivity and phase of the impedance tensor elements (Cagnard, 1953)-this includes frequency dependent and maplot results.

Schmucker (1987) $\rho^{*}$-z* parameters (frequency dependent results only)

Swift (1967) and Bahr (1988) skew and rotation angles including a multifrequency version of Bahr (1988) decomposition-Frequency dependent (F) and mapplot (M) results.

Local induction arrows Wiese (1962) (F)

Groom \& Bailey (1989) decomposition (F)

Chave \& Smith $(1994,1997)$ decomposition (F)

Becken \& Burhardt (2004) decomposition (F)

Phase ellipses(M) Inductive strength(Lezaeta \& Haak, 2003) (M)

Berdichevsky (M, F) and Weaver et al. (2000) invariants(F) 
Mohr circles(Lilley, 1998a,b) (F)

Output of a rotated .mtt file

\section{GDS}

Perturbation tensor elements

Perturbation arrows

Normal induction arrows

GDS strike angles

Mohr circles

\section{D.2 3D modelling program}

\section{D.2.1 mtd3fwd}

d3fwd is a program that computes magnetic and electric fields at the surface of a 3D electrical resistivity model illuminated by electromagnetic plane waves (Mackie \& Booker, 1999)

\section{D.2.2 d3_to_mt}

D3_to_mt (Mackie \& Booker, 1999) is a utility program to convert fields computed by d3fwd to magnetotelluric (MT) impedances, apparent resistivy and phase.

\section{D.2.3 d3togmt}

This program reads a 3D forward model file prepared for the modelling code of $\mathrm{d} 3 \mathrm{fwd}$ (Mackie \& Booker, 1999) and does the following: (i) Create a station file with coordinates to be used by emdisp (ii) Create data files that can be plotted using GMT (Wessel \& Smith, 1998) or (iii) Creates resistivity layer data files of the model that can be plotted with GMT.

\section{D.3 Data manipulating programs}

\section{D.3.1 Convert}

This is a Fortran-77 program written for this thesis-it reads magnetic observatory data from Alice spring and converts it to the göttinger binary saved format * ${ }^{*}$.goe (section 4.3) that can be read by the program select. 


\section{D.3.2 Calc}

Also written in Fortran-77 for the purpose of this thesis, it evaluates the MT transfer function at a station with an incomplete B-field by replacing the B-field at the station with that of a nearby station and correcting for any anomalous field between the stations.

\section{D.3.3 Gdsswap}

Gdsswap written by Leibecker (2000) is used to change the reference station of GDS data. This is necessary if during data analysis, a more suitable 1D site compared to the reference site at measurement time is discovered. For explicit expressions of the perturbation tensors after change of reference site, see Leibecker (2000). 


\section{E Phase tensor}

Regional phase information can be recovered directly from the observed galvanic distorted impedance tensor even where the regional conductivity structures are 3D. From equation 2.41, separating the complex impedance tensors into their real $(\mathrm{X})$ and imaginary $(\mathrm{Y})$ parts, one can write:

$$
\underline{\underline{Z}}=\underline{\underline{X}}+i \underline{\underline{Y}}
$$

and

$$
\underline{\underline{Z}}_{R}=\underline{\underline{X}}_{R}+i \underline{\underline{Y}}
$$

Hence, from equation 2.41:

$$
\underline{\underline{X}}=\underline{\underline{D}} \cdot \underline{\underline{X}}
$$

and

$$
\underline{\underline{Y}}=\underline{\underline{D}} \cdot \underline{\underline{Y}}
$$

The phase of a complex number is defined from the ratio of its real and imaginary parts; this can be generalized to a complex tensor. Therefore,the phase tensor can be defined using the relation

$$
\underline{\underline{\Phi}}=\underline{\underline{X^{-1}}} \cdot \underline{\underline{Y}},
$$

where $\underline{X}^{-1}$ is the inverse of the tensor $\underline{X}$ and $\underline{\Phi}$, the product of $\underline{X}^{-1}$ and $\underline{\underline{Y}}$ is real. The relationship between the phase tensors of the observed and regional impedance tensors is derived as follows:

$$
\begin{aligned}
\underline{\underline{\Phi}} & =\underline{\underline{X}}-1 \\
& =\underline{\underline{\underline{Y}}} \cdot \underline{\underline{X}})^{-1}(\underline{\underline{D}} \cdot \underline{\underline{Y}}) \\
& =\underline{\underline{X}}_{R}^{-1} \underline{\underline{D}}{ }^{-1} \underline{\underline{D}} \cdot \underline{\underline{Y}}=\underline{\underline{X}}_{R}^{-1} \cdot \underline{\underline{Y}} \\
& =\underline{\underline{\Phi}}_{R}
\end{aligned}
$$

Hence the observed and regional phase tensors are identical and are independent of the distortion tensor, $\underline{\underline{D}}$. The derivation makes no assumption about the nature or dimensionality of the regional conductivity structure. 


\section{References}

Adam, A. (2005), Graphite/graphitic rocks as cause of electric conductivity anomaly and their relationship to tectonics- a review. Acta Geol 175:361-411

Agarwal, A.K. \& Weaver, J.T.: (2000), Magnetic distortion of the magnetotelluric tensor: a numerical study. Earth planets Space, 52, 347-353,2000

Australian Bush Hospitality Pty,Ltd. Information about the geology of the Great Artesian Basin. http://www.diamantina-tour.com.au/outback_info/great_artesian_basin/great_ artesian_basin_geology.html

Almeida, E, Monteiro Santos, F, Mateus, A, Heise, W, Pous, J. (2005), Magnetotelluric measurements in SW Iberia: New data for the Variscan crustal structures. Geophys Res Lett 32, doi:10.1029/2005GL022596.

Archie, G.E.: 1942: The electrical resistivity log as an aid in determining some reservoir characteristics. Tran.AIME, 146, 54-67.

Babour, K. and Mosnier, Direct determination of the characteristics of the currents responsible for the geomagnetic anomaly of the rhinegraben. Geophys. J.R.astr. Soc.; 60:327-331, 1980 .

Bailey, R.C.and Edwards, R.N., The effect of source field polarization on geomagnetic variation anomalies in the British Isles. Geophys. J. R. astr.Soc, 45:95-104, 1976. 20, 36

Bahr, K.: 1997, Electrical anisotropy from conductivity distribution functions of fractal random networks in the crust: The scale effect of conductivity. GJI, 130, 649-660.

Bahr, K, Interpretation of the magnetotelluric impedance tensor: regional induction and local telluric distortion. J. Geophys. 62, 119-127 (1988).

Bahr, K, \& Simpson, F.,2002. Electrical Anisotropy Below Slow- and Fast-Moving Plates: Paleoflow in the Upper Mantle? Science, 295,1270-1272.

Banks, R.J, Data processing and interpretation in geomagnetic deep sounding. Phys. Earth Planet. Inter., 7:339-348, 1973. 21.

Banks, R.J, The use of equivalent current systems in the interpretation of geomagnetic deep sounding data. Geophys. J. R. astr. Soc,56:139-157, 1979. 20, 30, 125, 151.

Becken, M. \& Burkhardt H.: 2004, An ellipticity criterion in magnetotelluric tensor analysis. Geophysics Journal International, 159, 69-82

Berdichevsky, M.N.and Dmitriev, V.I.: 2008, Models and Methods of Magnetotelluric. Springer-Verlag Berlin Heidelberg, PP.119-121. 
Betts, P.G. \& Glies, D., The 1800-1100Ma tectonic evolution of Australia. Precambrian Research 144(2006) 92-125.

Betts, P.G., Giles D., Mark, G., Lister, G.S. Goleby B. R., Ailleres L.: 2006, Synthesis of the Proterozoic evolution of the Mt Isa Inlier. Australian Journal of Earth Sciences, 53:1, $187-211$.

Betts, P.G., Giles D., Lister G., Frick L.: 2002, Evolution of the Australian Lithosphere. Australian Journal of Earth Sciences, 49 661-695

Bibby, H.M., Caldwell, T.G., and Brown, C.(2005): Determinable and non determinable parameters of Galvanic distortion in magnetotellurics: Geophys.J.Int.(2005) 163, 915930.

Bierlein, F. \& Betts P.: 2004, The Proterozoic Mount Isa fault zone, northeastern Australia:is it really a ca $1.9 \mathrm{Ga}$ terrane-bounding suture? Earth and Planetary Science Letters, 225,279-294.

Boerner, D.E.; Kurtz R.; Craven, J.(1996), Electrical conductivity and Paleo-Proterozoic foredeeps. J Geophys Res 101:13775-13791.

Boerner, D.E.; Kurtz, R.; Craven, J.; Ross, G.; Jones, F.; Davis, W.: 1999, Electrical conductivity in the Precambrian Lithosphere of western Canada. Science, 283, 668-670.

Bott, M.H.P.: 1995, Mechanisms of rifting: Geodynamic modelling of continental rift systems. In: K.H. Olsen (ed.) continental rifts: Evolution, structure, tectonics. Developments in Geotectonics.

Brown, C., Tectonic interpretation of Regional conductivity Anomalies. Surveys in Geophysics 15: 123-157, 1994. Brown, C. \& Whelan, J.P., Terrane boundaries in Ireland inferred from the Irish Magnetotelluric Profile and other Geophysical data. Journal of the Geological Society 1995; v.152; p.523-534

Brown, D.A., Campbell, K.S.W and Crook, K.A.W.: 1968, The Geological Evolution of Australia and New Zealand. pergamon, Oxford.

Buckley, P.M.: 2001, Grasmere 1:100 000 Sheet, home to a few surprises. Minfo 70, $18-20$

Cagnard, L., 1953, Basic theory of the magnetotelluric method of geophysical prospecting. Geophysics, 18, 605-635.

Caldwell, T.G., Bibby, H.M. and Brown, C.: 2002, The magnetotelluric phase tensor-a method of distortional analysis for 3D regional conductivity structures: Abstract EM124, 16th Workshop on Electromagnetic Induction in the Earth, Santa Fe, New Mexico, USA.

Caldwell, T.G., Bibby, H.M. and Brown, C.: 2004, The magnetotelluric phase tensor: Geophys. J. Int.(2004) 158, 457-469

Calvert, A.J. and Clowes, R.M.:1990, 'Deep, High-Amplitude Reflection from a major shear zone above the Subducting Juan de Fuca Plate', Geology 18, 1091-1094. 
Campbell, W.H.: (1997), 'Geomagnetic pulsations' in S. Matsushita and W.H. Campbell (eds), physics of Geomagnetic Phenomena, 821-909, Academic Press, New York.

Campbell, W.H.: 1997, Introduction to geomagnetic fields. Cambridge University Press.

Campbell, W.H., Barton, C.E., Chamalaun, F.H., and Welsh, W., Quiet-day ionosphere currents and their application to upper mantle conductivity in Australia. Earth Planets, 50, 347-360, 1998. Carter, E.K., Brooks, J.H. \& Walker, K.R.: 1961, The Precambrian mineral belt of north-western Queensland. Bureau of Mineral Resources Bulletin, $\mathbf{5 1}$

Campbell, R.J. \& Haig, D.W., Bathymetric change during Early Cretaceous intracratonic marine transgression across the northeastern Eromanga Basin, Australia. Cretaceous Reaearch (1999) 20, 403-446.

Cayley, R.A., Korsch, R.J., Moore, D.H., Costelloe, R.D., Nakamura,A., Willman, C.E., Rawling, J.,Morand, V.J., Skladzien, P.B. \& O'shea, P.J., Crustal architecture of central Victoria: results from the 2006 deep crustal reflection seismic survey. Australian Journal of Earth Sciences (2011)58, (113-156).

Cengage Gale: 2003. eNotes.com. 2006. 21 Jul, $2011<$ http://www.enotes.com/earthscience/ supercontinents> "Supercontinents." World of Earth Science. Ed. K. Lee Lerner and Brenda Wilmoth Lerner.

Chakridi, R., Chouteau, M., Mareschal, M., A simple technique for analysing and partly removing galvanic distortion from the magnetotelluric impedance tensor; application to Abitibi and Kapuskasing data (Canada), Geophys. J. Int. 108 (3) (1992) 917-929.

Chamalaun, F.H.: 1985, Geomagnetic deep sounding experiment in the central Flinders Ranges of South Australia. Phys. Earth Planet. Int.37, 174-182.

Chamalaun, F.H.: 1986, Extension of the Flinders Ranges Anomaly: Exploration Geophysics $\mathbf{1 7}, 31$

Chamalaun, F.H. and Cuneen, P.: 1990, The Canning Basin geomagnetic induction anomaly: Australian Journal of Earth Sciences 37,401-408

Chamalaun, F.H. and Barton, CE.: 1993, The large-scale electrical conductivity structure of Australia: Journal of Geomagnetism and Geoelectricity 45, 1209-1212

Chamalaun, F.H., Lilley, F.E.M. and Wang, L.J.: 1999, Mapping the Carpentaria conductivity Anomaly in northern Australia. Physics of the Earth and Planetary Interiors 116, $105-115$

Chave, A.D.\& smith, J.T.: 1994, On electric and magnetic galvanic distortion tensor decompositions, J.geophys.Res., 99(B3), 4669-4682.

Chen, P.F. \& Fung, P.C.W., 1985, Significance of the sign changing of the imaginary arrows in geomagnetic induction investigation. Geophys. J.R. astr. Soc. 80,257-263

Chivas, A. R., Garcíaa, A., van der Kaarsb,S., Couapela, M. J. J., Holta,S., Reevesa, J. M., Wheelera, D. J., Switzera, A. D., Murray-Wallacea, V., Banerjeea, D., Pricea, D. M. , Wanga, S. X., Pearsona, G., Edgard, N. T., Beauforte, L., De Deckkerf, P., Lawsong, E. and Cecilh, B. Sea-level and environmental changes since the last interglacial in the Gulf of Carpentaria, Australia. Quaternary International 83-85 (2001) 19-46. 
Clitheroe, G., Gudmundsson, O.\& Kennett, B.L.N.: 2000b, Sedimentary and upper crustal structure of Australia from receiver functions. Australian Journal of Earth Sciences 47, 209-216.

Collins, C., Drummond, B. and Nicoll, M.: 2003, Crustal thickness patterns in the Australian continent. In R.Hillis; R.Müller, editors, Evolution and dynamics of the Australian plate, volume 22 of Geological Society of Australia Spec. Pub.,pp.121-128.

Coney, P. J., Alaster E., Richard, H., Fiona, M., and Donal, W., The regional tectonics of the Tasman Orogenic System, Eastern Australia. Journal of structural Geology 1990, $12,519-543$

Crawford, A.J.; Meffre, S.; Symonds, P.A.: 2003, 120 to 0 Ma tectonic evolution of the southwest pacific and analogous geological evolution of the 600 to $220 \mathrm{Ma}$ Tasman fold belt system. In R. Hillis; R.Müller, editors, Evolution and dynamics of the Australian plate, Geol.Soc.Australia Spec.Publ. 22, and Geol.Soc. America Spec.Pap.372 (2003),383-403

Day, R.W., Murray, C.G., and Whitaker, W.G.: 1978, The eastern part of Tasman orogenic Zone. Tectonophysics, 48:327-364.

Day, R.W., Whitaker, W.G., Murray, C.G. Wilson, I.H and Grimes, K.G.: 1983, Queensland Geology.Geol. Surv. Qld.Publ., 383

Debayle, E. \& Kennett, B.: 2000, The Australian continent upper mantle: Structure and deformation inferred from surface waves. Journal of Geophysical Research, 105(B11), 25423-25450.

Denham, D.: 1991, Shear wave crustal models for the Australian continent. In: Drummond B.J.ed. The Australian Lithosphere, pp.59-66. Geophysical Society of Australia Special publication 17.

Denmead, A.K., Tweedale, G.W. \& Wilson, A.F.: 1974, The Tasman Geosyncline- a Symposium. Geological Society of Australia, Queensland Division, Brisbane.

Direen, N.G.: 1998, The palaeozoic Koonenberry Fold and Thrust belt, far western New South Wales: a case study in applied gravity and magnetic modelling. Exploration Geophysics 29,330-339.

Direen, N.G. and Crawford, A.J., Australian Journal of Earth Sciences(2003)50, 491502.

Drummond, B.J., Muirhead, K.J., Wellman, P. and Wright, C.: 1991, A teleseismic traveltime residual map of the Australian continent. BMR Journal of Australian Geology and Geophysics 11, 101-105.

Encyclopedia Britannica Online:http://www.britannica.com/EBchecked/topic/552982/solarquiet-day-variation

Edwards, R.E. and Nabighian, M.N.:(1981), Extensions of the magnetometric resistivity (MMR) method. Geophysics 46:459-460.

Egbert, G.; Booker, J.R.: 1986, Robust estimation of geomagnetic transfer functions. Geophysical Journal of the Royal astronomical Society, 87, 173-194. 
Embleton, B.J.J.: 1984, Past global settings, continental Palaeomagnetism. In: J.J. Veevers (Editor), Phanerozoic Earth History of Australia. Claredon Press, Oxford, PP.11-16.

Evans, A.D. Davids, The palaeomagnetically viable, long-lived and all-inclusive Rodinia supercontinent reconstruction. Geological Society, London, Special Publications 2009; v. 327; p. 371-404.doi:10.1144/SP327.16

Everett, J.E \& Hyndman, R.D.: 1967, Geomagnetic variations and electrical conductivity structure in south-western Australia, Phy. Earth planet. int., 1, 24-34.

Finlayson, D.M. \& Leven, J.H., Lithospheric structurees and possible processes in Phanerozoic eastern Australia from deep seismic investigations. Tectonophysics, 133(1987) 199215 .

Finlayson, D.M., Wake-Dyster, K.D., Leven, J.H., Johnstone, D.W., Murray, C.G, Harrington, H.J., Korsch, R.J and Willman P., Seismic imaging of major tectonic features in the crust of Phanerozoic eastern Australia. Tectonophysics, 173(1990) 211-230.

Fishwick, S., Kennett, B. and Reading, A.: 2005, Contrasts in lithospheric structure within the Australian craton-insights from surface wave tomography. Earth and Planetary Science Letters, 231, 163-176.

Fluche, B.:1983, Gemagnetic depth sounding and magnetotellurics in the Hessian Depression, Diploma thesis, Inst, Geoph., University of Göttingen, Germany (in German).

Foster, D. A. and Gray, D. R., Evolution and structure of the Lachlan Fold Belt (Orogen) of eastern Australia. Annual Review of Earths Planetary sciences 2000. 28, 47-80

Frakes, L.A., Burger,D., Apthorpe, M., Wiseman, J., Dettmann, M., Alley, N., Flint, R., Gravestock, D., Ludbrook, N., Backhouse, J., Skwarko, S., Scheibnerova, V., McMinn, A., Moore, P.S, Bolton, B.R., Douglas, J.G, Christ,R., Wade,M., Molnar, R.E., Mcgowran,B., Balme, B.E. and Day, R.A.: 1987, Australian Cretaceous shorelines, stage by stage. Palaeogeogr., Palaeoclimatol., Palaeocol., 59:31-48

Fraser, A.R., Moss, F.J.\& Turpie, A.: 1976, Reconnaissance gravity survey of Australia. Geophysics 41, 1337-1345.

Frost, B.R.: 1991, 'Magnetic Petrology; Factors that Control the Occurrence of Magnetite in crustal Rocks', Rev. Mineralogy 25, 489-509

GABCC: 1998, Great Artesian Basin resource study summary. http://www.gab.org.au/.Great Artesian Basin Consultative Council

GABCC: 2000, Great Artesian Basin; strategic management plan. The Great Artesian Basin Consultative Council.

Gatzemeier, A.: 2002, Elektrische Anisotropie durch ausgerichtete Olivinkristalle im oberen Mantle in Mitteleuropa: Magnetotellurische Array-Messungen und ein Ansatz zum vergleich mit seismisher Anisotropie. Ph.D. thesis, Universität Göttingen.

Giles, D., Betts, P., Ailleres, L., Hulscher, B., Hough, M., Lister, G.: 2006, Evolution of the Isan Orogeny at the southeastern margin of the Mt Isa Inlier. Australian Journal of Earth Sciences, 53, 91-108. 
Giles, D., Betts, P., Lister, G.: 2004, 1.8-1.5Ga links between the north and south Australian cratons and the early-middle proterozoic configuration of Australia. Tectonophysics, 380, 27-41.

Gough, D.I.: 1983, Electromagnetic geophysics and global tectonics. Journal of Geophysical Research 88, 3367-3377.

Gough, D.I., McElhinny, M.W. and Lilley, F.E.M :(1974), A magnetometer array study in southern Australia. eophys. J.R. astr. Soc.36, 345-362

Gough, D.I., McElhinny, M.W. and Lilley, F.E.M :(1972). A polarization sensitive magnetic variation anomaly in south Australia. Nature, physical sciences 239, 88-91.

Graeme, D. McDonald, Kenneth, D. Collerson and Peter, D. Kinny, Late Archean and Early Proterozoic crustal evolution of the Mount Isa block, northwest Queensland, Australia. Geology 1997; 25; 1095-1098, doi: 10.1130/0091-7613 (1997) 025<1095:LAAEPC > 2.3.CO; 2

Gray, D.R. \& Foster, D.A.: 2004, Tectonic evolution of the Lachlan Orogen, southeast Australia: historical review, data synthesis and modern perspectives. Australian Journal of Earth Sciences, 51, 773-817.

Groom, R. W. and Bailey, R. C., Decomposition of the magnetotelluric impedance tensor in the presence of local three-dimensional galvanic distortion. J. Geophys. Res. 94, 1913-1925 (1989).

Groom, R. W. and Bailey, R. C., Some effects of multiple lateral inhomogeneities in magnetotellurics. Geophysical Prospecting, 37:697-712, 1989b.

Groom, R. W.\& Bahr, K.: 1992, Corrections for near surface effects: Decomposition of magnetotelluric impedance tensor and scaling corrections for regional resistivities: a tutorial, Surv. Geophys., 13, 341-379.

Gunn, P.J., Milligan, P., Mackey, T., Murray, A., Maidment ,D., and Haren, R.: 1997, Geophysical mapping using the national airborne and gravity datasets; an example focusing on Broken Hill. AGSO Journal of Australian Geology and Geophysics 17, 127-136.

Haak, V. and Hutton, R.: 1986, Electrical resistivity in continental lower crust. Geological Society, London, Special Publications 1986; v. 24; p. 35-49

Hanekop, O.: 2006, PhD thesis p.89-90. large scale resistivity surveys combining magnetic and magnetotelluric observations -Examples from central Australia.

Harrington, H.J.:1974, The Tasman Geosyncline in Australia.In: Denmead A.K., Tweedale G.W. and Wilson A.F. eds. The Tasman Geosyncline-A Symposium in Honour of Profesor Dorothy Hill, pp.383-407. Geological Society of Australia, Quessnsland Division, Brisbane.

Heinson, G.S. and White, A.: 2005, Electrical resistivity of the northern Australian lithosphere: crustal anisotropy or mantle heterogeneity? Earth Planet. Sci. Lett., 232, 157170.

Heise, W. \& Pous, J.: 2003, Anomalous phases exceeding $90^{\circ}$ in magnetotellurics: anisotropic model studies. 
Heise, W., Caldwell, T.G., Bibby, H.M.,\& Brown, C., Anisotropy and phase splits in magnetetolluric. Physics of the Earth and Planetary interiors 158 (2006) 107-121.

Hill, D.: 1951, Geology. In:Mack G.ed.Handbook of Queensland, pp.13-24. Australian Association for the Advancement of Science, Brisbane.

History.com: www.history.com/topics/australia

Hitchman, A., Lilley, F.E.M., Campbell, Chamalaun, F., and Barton C.: 1998, The magnetic daily variation in Australia: dependence of the total-field signal on latitude: Exploration Geophysics 29, 428-432.

Hitchman, A.P., Milligan, P.R., Lilley, F.E.M., White, A., and Heinson, G.S.: 2000. The total-field geomagnetic coast-effect: the CICADA97 line from deep Tasman Sea to inland New South Wales. Exploration Geophysics 31

Honkura Yoshimori, Electrical conductivity anomalies in the Earth. Geophysical Surveys 3 (1978) 225-253

Ichiki M., Baba K., Toh H. Fuji-ta K. An overview of electrical conductivity structures of the crust and upper mantle beneath the north-western Pacific, the Japanese Islands, and continental East Asia. Gondwana Research 16 (2009) 545-562

Jödicke, H.: (1992), Water and graphite in the earth's crust- an approach to interpretation of conductivity models. Surv Geophys. 13:381-407.

Jones, A.G.: (1983), The problem of current channelling: a critical review. Geophysical Surveys 6: $79-122$.

Jones, A.G. \& Groom, R.W., Strike-angle determination from the magnetotelluric impedance tensor in the presence of noise and local distortion: rotate at your peril! Geophys. J. Int. (1993).

Jording, A., Ferrari, L. Arzate, J., Jödicke, H., Crustal variations and terrane boundaries in southern Mexico as imaged by magnetotelluric transfer functions. Tectonophysics $327(200) 1-13$.

Keller, G.V. and Frischknecht, F.C.: 1970, Electrical Methods inGeophysical Prospecting. Pergamon Press, New York, N.Y., 517 pp.

Kellett, R.L, Mareschal, M., Kurtz, R.D, A model of lower crustal electrical anisotropy for the Pontiac subprovince of the Canadian Shield, Geophys.J. Int. 111 (1) (1992) 141-150.

Kellett, R.L., White, A., Ferguson, I.J. \& Lilley, F.E.M.:1988, Geomagnetic Fluctuation Anomalies Across the Southeast Australian Coast. ASEG/SEG Conference-Adelaide Pages 294-297

Kennett, B.L.N., Seismic structure in the mantle beneath Australia, Geol. Soc. Australia Spec. Publ.22, and Geol. Soc. America Spec. Pap. 372 (2003), 7-23.

Kennett, B.L.N., Fishwick, S., Reading, A.M. and Rawlinson, N.: 2004, Contrasts in mantle structure beneath Australia: relation to Tasman lines? Australian Journal of Earth Sciences, 51, 563-569. 
Kirkegaard, A.G., 1974, Structural elements of the northern part of Tasman Geosyncline. In: A.K. Denmead, G.W. Tweedale and A.F Wilson(Editors), The Tasman Geosycline. Geological Society of Australia(Qld.Div.), Brisbane, PP.47-63.

Kivelson, M.G. and Russell, C.T., 1995, Introduction to Space Physics, Page 407. Cambridge: Cambridge university Press.

Korch, R.J., Harrington, H.J., Wake-Dyster, K.D., O'Brien, P.E. and Finlayson, D.M., 1988, Sedimentary basins peripheral to New England Oregen: their contribution to understanding New England tectonics. In: J.D. Kleeman (Editor), New England Orogen, Tectonic and Metallogenesis. Department of Geology and geophysics. University of New England, pp.134-140.

Korja, T., Electrical conductivity of the Lithosphere-Implication for the Evolution of the Fennoscandian Shield. Geophysica (1997), 33(1), 17-50.

Leibecker, J.: 2000, Electromagnetische Arraymessungen im Rheinischen Schiefergebirge: Modell der elektrischen Leitfähikeit der Erdkruste und des oberen Mantels mit Verbindungen zum Eifelvukanismus (in German). Ph.D. Thesis, Universität Göttingen

Leibecker, J., Gatzemeier, A.; Hönig, M.; Kuras, O.; Soyer, W.: 2002, Evidence of electrical anisotropic structures in the lower crust and upper mantle beneath the Rhenish Shield. Earth and Plantary Science letters 202 (2002) 289-302.

Lezaeta, P.: 2001, PhD Dissertation. Distortion Analysis and 3-D Modelling of Magnetotelluric Data in the Southern Central Andes

Lezaeta, P. \& Haak, V.: 2003, Beyond magnetotelluric decomposition: Induction, current channelling and magnetotelluric phases over $90^{\circ}$. Journal of Geophysical Research, 108, EPM 4 1-20.

Li, Z. X. \& Powell, C. McA.: 2001, An outline of the palaeogeographic evolution of the Australasian region since the beginning of the Neoproterozoic. Earth Science Reviews 53, $237-277$.

Lilley, F.E.M., Geomagnetic Field Fluctuations over Australia in Relation to Magnetic Surveys. Bull.Aust.Soc.Explor.Geophys.v.13 no.3,68-76. 1982

Lilley, F.E.M. \& Arora, B.R.: 1982, The sign convention for quadrature Parkinson arrows in geomagnetic induction studies, Rev. Geophys. Space phys., 20, 513-518.

Lilley, F.E.M., Bennet, D.J., 1972, An array experiment with magnetic variometers near the coasts of south-east Australia. geophys.J.R. Astron. Soc.29, 49-64.

Lilley, F.E.M.: 1998a, Magnetotelluric tensor decomposition: Part 1, theory for basic procedure. Geophysics, 63(6), 1885-1897.

Lilley F.E.M.: 1998b, Magnetotelluric tensor decomposition: Part 2, examples of a basic procedure. Geophysics, 63(6), 1898-1907.

Lilley, F.E.M., Hitchman, A.P., and Wang, L.J.: 1999, Time-varying effects in magnetic mapping: Amphidromes, doldrums and induction hazard: Geophysics 64, 1720-1729. 
Lilley, F.E.M., Wang L.; Chamalaun, F.; Ferguson, I.: 2003, The Carpentaria electrical conductivity anomaly, Queensland, as a major structure in the Australian Plate. In R.Hillis; R.Müller, editors, Evolution and Dynamics of the Australian Plate, Geol. Soc. Australia Spec. Publ.22, and Geol. Soc. America Spec. Pap. 372 (2003), 141-428.

Lilley, F.E.M., Hitchman, A.P, Milligan, P.R., and Pedersen, T. Sea-surface observations of magnetic signals of Ocean swells. Geophys. J. Int.(2004) 159,565-572.

Lilley, F.E.M., Weaver, J.T., Phases greater than $90^{\circ}$ in MT data: Analysis using dimensionality tools, J. Appl. Geophys. (2009), doi:10.1016/j.jappgeo.2009.08.007

MacCready, T.: 2006, Structural cross-section based on the Mt Isa deep seismic transect. Australia Journal of Earth Sciences, 53, 5-26.

Mackie, R.L., Booker, J.: 1999, Documentation for mtd3fwd and d3-to-mt. Unpublished user documentation. GSY-USA Inc., 2261 Market St., Suite 643, San Francisco, CA 94114.

Mackie, R.L., Smith, J.T. \& Madden, T.R.: 1994, Three-dimensional electromagnetic modelling using finite difference equation: the magnetotelluric example, Radio Sci., 29, 923-935.

Mareschal, M., Kellett, R.L., Kurtz, R.D., Ludden, J.N., Ji,S. \& Bailey, R.C., Archaean cratonic roots, mantle shear zones and deep electrical anisotropy, Nature (Lond.) 375(6527) (1985) 134-137.

Marquis, G. and Hyndman, R.D, Gephysical support for aqueous fluids in the deep crust: Seismic and electrical relationships. Geophys. J. Int.(1992) 110, 91-105.

Marti, A., Queralt P., Ledo J.: 2005, Improving Bahr's invariant parameters using WAL approach. Geophysical Journal International, 163, 38-41.

Matsushita, S.:(1967), 'Solar quiet and Lunar daily variations fields' in S. Matsushita and W.H. Campbell (eds), physics of Geomagnetic phenomena, 301-424, Academic Press, New York.

McNeill, J.D and Labson, V.F.: 1991, Geological mapping using VLF radio field. In: M.N. Nabighian, Editor, Electromagnetic Methods in Applied Geophysics II, SOC. Exp. Geophysic.(1991),pp.521-640.

McDougall, M.J.: 1996, Resistivity profiles and structural models over the western margin of the Eromanga Basin, northwest Queensland. MSc thesis, Monash University, Melbourne (unpublished)

Meissner, R. and Wever, Th.:1992, 'The possible role of fluids for the structuring of the continental crust', Earth Sci. Rev.32, 19-32.

Miensopust, M.P.,Jones, A.G., Muller,M.R., Garcia, X. \& Evans,R.L. Lithospheric structures and Precambrian terrane boundaries in northeastern Botswana revealed through magnetotelluric profiling as part of the Southern African Magnetotelluric Experiment. JOURNAL OF GEOPHYSICAL RESEARCH, VOL. 116, B02401, 2011.

Milligan, P.,2010. https://www.ga.gov.au/products/servlet/controller?event=GEOCAT_DETAILS \&catno $=70282$ 
Milligan, P.R. \& Barton, C.E., 1997. transient and induced variations in aeromagnetic, Records of Australian Geological Survey organization Vol., 27, AGSO, Canberra.

Milligan, P.R, Petkovic,P. and Drummond, B.J. Potential-field datasets for Australia region: their significance in mapping basement architecture. Geological societal Australia Special Publication 22 and Geological societal America Special Pap.372 (2003)

Milligan, P.R., R. Franklin, D. Ravat (2004). A new generation of Magnetic Anomaly Grid Database of Australia (MAGDA) - use of independent data increases the accuracy of long wavelength components of continental-scale merges, Preview, 113, 25-29.

Moorkamp, M., 2003. Elektrische Anisotropie im oberen Mantel (in German). Masters Thesis, Institut für Geophsik, Universität Göttingen.

Muirhead, K.J.\& Drummond B.J. 1991. The seismic structure of the lithosphere under Australia and its implicatins for continental plate tectonics. In: Drummond B.J. ed. The Australian Lithosphere, pp.23-40. Geological Society of Australia Special Publication 17.

Murray A.S., Morse M., Milligan P.R. \& Mackey T. 1997. Gravity Anomaly Map of the Australian Region, scale 1:5000 000(2nd edition).Australian Geological Survey Organisation, Canberra.

Murray, A.S. \& Petkovic, P.: 2001, Digital gravity grid of the Australian region, CD-ROM. AGSO-Geoscience Australia, Canberra.

Murraya, C. G.\& Scheibner, E. 1989, Regional geological interpretation of a digital coloured residual. Bouguer gravity image of eastern Australia with a wavelength cut-off of $250 \mathrm{~km}$. Australian Journal of Earth Sciences,36,423-449.

Myers, J.S; Shaw R.D; Tyler I.M; 1996, Tectonic evolution of Proterozoic Australia. Tectonics,15(6),1431-1446.

Neudert, M.K. \& Russell, R.E.: 1981, Shallow water and hypersaline features from the middle Proterozoic Mount Isa Sequence. Nature, 293, 284-286.

Nover, G., Stoll, JB, von der Gönna J.: (2005), Promotion of graphite formation by tectonic stress- a laboratory stress experiment. Geophys. J Int 160:1059-1067.

O’Dea, M.G., Lister, G.S.,Maccready, T., Betts, P.G., Oliver, N.H.S, Pound, K.S., Huang, W., and Valenta, R.K., Geodynamic evolution of the Proterozoic Mount Isa terrain. Geological Society, London, Special Publications 1997; v.121;p.99-122.

Ogawa, Y., On two-dimensional modelling of magnetotelluric Field data. Surveys in Geophysics 23: 251-257, 2002

O'Reilly, S.Y. Griffin, W.L., Poudjom Djomani, Y.H. \& Morgan, P. 2001. Are lithospheres forever? Tracking changes in sub continental lithopheric mantle through time. GSA Today, 11 (April), 4-10.

Page, R.W., Bell, T.H.: 1986, Isotopic and structural responses of granite to successive deformation and metamorphism. J. Geol. 94, 365-379. 
Page, R.W., Sun, Shen-Su\& Carr, G.: 1994, Proterozoic sediment-hosted lead-zinc-silver deposits in northern Australia-U-Pb zircon and $\mathrm{Pb}$ isotopic studies. Geological Society of Australia Abstracts, 37, 334-335.

Page, R.W. and Williams, I.S., Age of the Barramundi Orogeny in north Australia by means of ion microprobe and conventional U-U-Pb zircon studies. Precambrian Research, 40/41 (1988) 21-36.

Parker, E.N.: (1958), Dynamics of interplanetary gas and magnetic field. Astrophys. J.128: $664-676$

Parkinson, W.D., Introduction to Geomagnetism. Scottish Academic Press, 1983. 5, 12, $20,24,81$.

Parkinson, W.D.: 1959, Direction of rapid geomagnetic fluctuations. Geophysical Journal, 2, 1-14.

Parkinson, W.D, 1962, The influence of continents and Oceans on geomagnetic variations, Geophs.J.R. astr. SOC.,6, 441-449

Parkinson, W.D \& Jones, F.W., The Geomagnetic Coast Effect. Reviews of Geophysics and Space physics, 1979, 17.

Peter T. Harris, Andrew D.Heap, Theodore Wassenberg and Vicki Passlow, Submerged Coral reefs in the Gulf of Carpentaria, Australia. Marine Geology 207,(2004) 185-191.

Popov, A.M., Kiselev, and V.V. Mordvinova, Geodynamic interpretation of crustal and upper mantle electrical conductivity anomalies in Sayan-Baikal Province. Earth Planets Space, 51, 1079-1089, 1999.

Rasmussen, T.M., 1988, Magnetotellurics in south-western Sweeden; evidence for electrical anisotropy in the lower crust? J.geophys. Res.,93,7897-7907.

Ritter, O., Weckmann U, Vietor, T., Haak, V.:(2003b), A magnetotelluric study of the Damara belt in Namibia:1. Regional scale conductivity anomalies. Phys Earth Planet Int 138:71-90.

Ritter, P., Separation of local and regional information in geomagnetic response functions using hypothetical event analysis, PhD thesis, University of Edinburg, Edinburg, 1996.

Ritter, P \& Banks, R.J. :(1998), Separation of local and regional information in distorted GDS reponse functions by hypothetical event analysis. Geophysics J.Int. 135:923-942.

Ritz, M. \& Robineau B.: 1986, Crustal and upper mantle electrical conductivity structures in West Africa: Geodynamic implications. Tectonophysics, 124:115-132.

Scheibner, E. and Veever, J.J.: 2000.19, Tasman Fold Belt System. In: Veevers J.J. ed. Billion-year Earth History of Australia and Neighbours in Gondwanaland, pp. 154-234. GEMOC Press, Sydney.

Scheibner, E.: 1974, Fossil fracture zones(transform faults), segmentation, and correlation problems in the Tasman Fold Belt system.In: Denmead A.K., Tweedale G.W. and Wilson A.F. eds. The Tasman Geosyncline-A Symposium in Honour of Profesor Dorothy Hill, pp.65-98. Geological Society of Australia, Quessnsland Division, Brisbane. 
Scheibner, E.: 1996, Tasman Fold Belt system. In: Scheibner E. and Basden H. eds. geology of New South Wales-Synthesis, Vol.1. Structural Framework, pp.45-67. Geological Survey of New South Wales Memoir, Geology 13(1).

Scheibner, E. :1998. 11, Introductory Notes. In: Scheibner E. and Basden H. eds. Geology of New South Wales-synthesis, Vol.2. Geological Evolution, pp.3-21. Geological Survey of New South Wales Memoir, Geology 13(2).

Schmucker, U., 1970, Anomalies of geomagnetic variations in the Southern United states: Univ. of California Press, Berkley.

Schmucker, U.: 2003, Horizontal spatial gradient sounding and geomagnetic depth sounding in the period range of daily variations. 20. Kolloquium Elektromagnetische Tiefenforschung, Königstein, 29.09.-3.10.2003, Hrsg.: A. Hördt und J. B. Stoll

Selway, K., Heinson, G., Hand, M.:2006, Electrical evidence of continental accretion: Steeply-dipping crustal-scale conductivity contrast. Geophysical Research Letters, 33, doi:10.1029/2005GL025328.

Senior, B.R. \& Habermehl, M.A.: 1980, Structure, hydrodynamics and hydrocarbon potential, central Eromanga Basin, Queensland, Australia. BMR J. Aust. Geol. Geophys., $5,47-55$.

Shaw, R.D., Wellman P., Gunn p.J, Whitaker, A.J., Tarlowski, C. \& Morse M.P.: 1996, Guide to using the Australian crustal elements map. Australia Geological Survey Organisation Record 1996/30.

Siemon, B., An interpretation technique for superimposed induction anomalies. Geophys. J. Int.(1997) 130, 73-88

Simons, F., Ziehuis A. and van der Hilst, R.:1999, The deep structure of the Australian continent from surface waves tomography.Lithos, 48, 17-43.

Simons, F.and van der Hilst, R., 2002, Age-dependent seismic thickness and mechanical strength of the Australian lithosphere. GRL,29(11),24.

Simpson, F. and Bahr, K.: 2005, Practical Magnetotellurics.Cambridge: Cambridge University Press.

Simpson, F.: 2001, Resistance to mantle flow inferred from the electromagnetic strike of the Australian upper mantle. Nature, 412, 632-635.

Simpson, F.; Tommasi, A., 2005, Hydrogen diffusivity and electrical anisotropy of a peridotite mantle. Geophysical Journal international, 160(3), 791-803.

Singer, B.S., 1992, Correction for distortions of magnetotelluric fields: Limits of validity of the static approach, surv. Geophys.J. Int., 122, 219-226.

Singh, B.P., Geomagnetic Sounding of Conductivity Anomalies in the Lower Crust and Upper Mantle. Geophysical Surveys 4 (1980)71-87. 0046-5763/80/0041-0071\$02.55.

Smith, W.D.: 1969, Penecontemporaneous faulting and its likely significance in relation to Mount Isa ore deposition. Special Publications of the Geological Society of Australia, 2, 225-235. 
Smith, J.T., 1995, Understanding telluric distortion matrices, Geophys. J.Int., 122, 219226 .

Smith, J.T., Estimating galvanic-distortion magnetic fields in magnetotellurics, Geophys. J.Int. (1997) 130, 65-72.

Southgate, P.N.: 2000, Introduction. Carpentaria-Mt Isa Zinc Belt: basement frame work, chronostratigraphy and geodynamic evolution of Proterozoic successions. Australia journal of the Geological Society of Australia 30, 1-16.

Soyer, W.; Brasse H.: 2001, A magneto-variation array study in the central and southern Andes of N Chile and SW Bolivia. Geophysical Research Letters, 28, 3023-3026..

Spence, A.G. \& Finlayson, D.M.:1983, The resistivity structure of the crust and upper mantle in the central Eromanga Basin, Queensland, using magnetotelluric techniques, Australian Journal of Earth Sciences (1980), 30 1-16

Steveling E.: 2000, Rap operating instructions for long period MT dataloggers. Technical report, University of Göttingen. V.4.08.

Steveling, E., Leven, M.: 1992, Ein Datenlogger für niederfrequente geophysikalische Messugen. 14. Kollquium zur eletromagnetischen Tiefenforschung in Borkheide vom 2529.5.1992. Deutsche Geophysikalische Gesellschaft.

Sternberg, B.K., Washburne, J.C. \& Pellerin, L., 1988, Correction for static shift in magnetotellurics using transient electromagnetic soundings, Geophysics, 53, 1459-1468

Swift, C.: 1967, A magnetotelluric investigation of an electrical conductivity anomaly in the south western United States. Ph.D. thesis, Cambridge, MA.

Tauber, S, Banks, R, Ritter, O, Weckmann U(2003). A high resolution magnetotelluric survey of the Iapetus Suture Zone in southwest Scotland. Geophys J Int 153:548-568.

Taylor John, R. An Introduction to Error Analysis, Universioty Science Books, (1997), PP.73-75.

Telford, WM, Geldart LP, Sheriff RE, Keys DA :(1976), Applied geophysics. Cambridge University Press, Cambridge.

Tournerie, B., Chouteau, M. and Marcotte, D., Magnetotelluric static shift: Estimation and removal using the cokriging method. Geophysics, Vol.72, No.1 (January-February 2007); P.F25-F34, 11Figs., 1Table. 10.1190/1.2400652.

Tracey, R., Bacchin, M, \& Wynne, P.: 2008, In preparation. AAGD07: A new absolute gravity datum for Australian gravity and new standards for the Australian National Gravity Database. Exploration Geophysics.

Utada, H. \& Munekane, H., 2000, On galvanic distortion of three dimensional magnetotelluric impedances, Geophys.J.Int., 140, 385-398.

Vaughan, A.P.M., Leat, P.T. \& Pankhurst, R.J (eds): 2005, Investigating the deep structure of terranes and terrane boundaries: insights from earthquake seismic data. Terrane Processes at the Margins of Gondwana. Geological Society London, Spec.Publ. 246, 293-303. 
Veevers, J.J. editor: 1984, Phanerozoic Earth History of Australia. Clarendon Press, Oxford,pp.351-364.

Veevers, J.J.:1990, Tectonic-climatic supercycles in the bilion-year plate-tectonic: Permian Pangean icehouse alternates with cretaceous dispersed-continents Greenhouse.Sediment. Geol. 68:1-16.

Veevers, J.J., and McElhinny M.W.,The Separation of Australia from other continents, Earth Sci.Rev., 12, !39-159, 1976.

Veevers, J.J. \& Powell, C.McA.,1984a. Uluru and Adelaidean regimes. In: J.J.Veevers (Editor),Phanerozoic Earth History of Australia. Clarendon Press, Oxford,pp.270-289.

Walter, M.R., Veevers, J.J., Calver, C.R. and Grey K., Neoproterozoic stratigraphy of the Centralian Superbasin, Australia, Precambrian Res., 73,173-195, 1995.

Waltermann, J., Beobachtung korrelieter ULF-Fluktuation im erdmagnetischen Feld und im Phasenweg ionosphärischer HF-Sondierungen, Ph.D. Thesis. Thesis, Institut für Geophysik, Universität Göttingen, 1984.

Wang, L.; Lilley, F.; Chamalaun, F.,1997. Large-scale Electrical Conductivity Structure of Australia from Magnetometer Arrays. Exploration Geophysics 28, 150-155.

Watson, E.B. \& Brenan, J.B.; 1987, 'Fluids in the Lithosphere, 1, Experimentally Determined Wetting Characteristics of $\mathrm{CO}_{2}-\mathrm{H}_{2} \mathrm{O}$ fluids and their Implications for Fluid Transport, Host-Rock Physical Properties and Fluid Inclusion Formation', Earth Planet. Sci. Lett.89, 3991-4358.

Weaver, J.T. and Agarwal,A.K. Is addition of induction vectors meaningful? Phys. Earth Planet. Inter., 65:267-275, 1991. 20, 139.

Weaver, J.T., Agarwal, A.K., \& Lilley, F.E.M.: 2006, The relationship between the magnetotelluric tensor invariants and the phase tensor of Caldwell, Bibby and Brown, Explor. Geophys.,37, $261-267$.

Weaver, J.T., Agarwal, A.K., Lilley, F.E.M.: 2003, The relationship between the magnetotelluric tensor invariants and the phase tensor of Caldwell, Bibby and Brown. In: Macnae, J., Liu, G. (Eds.), Three-Dimensional Electromagnetics III. No. 43 in Paper. Australian Society of Exploration Geophysicists, pp. 1-8.

Weaver, J.T., Agarwal, A.K., \& Lilley, F.E.M.: 2000, Characterization of the magnetotelluric impedance tensor in terms of its invariants, Geophys. J.Int., 141,321-336.

Weckmann, U.; Rtter, O.; Haak, V.: 2003, A magnetotelluric study of the Damara Belt in Namibia 2. MT phases over $90^{\circ}$ reveal the internal structure of the waterbergrg fault /omaruru lineament. Physics of the Earth and planetary interiors, 138, 91-112.

Wellman, P.: 1976, Gravity trends and the growth of Australia: a tentative correlation. Journal of the Geological Society of Australia 23, 11 - 14.

Wessel, P. \& Smith W., 1998, New improved version of the generic mapping tools released. EOS Trans., 79, 579, http://gmt.soest.hawaii.edu/. 
Whellams, J.M., 1996, Spatial inhomogeneity of geomagnetic fluctuations fields and their influence on high resolution aeromagnetic surveys: PhD thesis, Flinders University, South Australia.

White, A. \& Polatajko, O.W. (1978), 'The coast effect in geomagnetic variations in south Australia', J. Geomag. Geoelectr.30,109-120.

Whiteley, R.J. \& Pollard, P.C.: 1971, A combined deep resistivity and magnetotelluric sounding in the Eromanga Basin, Queensland.Search 2, 103-105.

Wiese, H. :(1962), Geomagnetische Tiefensondierung. Teil II: Die Streichrichtung der Untergrundstrukturen des elektrischen Widerstandes, erschlossen aus geomagnetischen variationen. Geofis. Pura et Appl. 52: 83-103.

Wiese, H. :(1965), Geomagnetische Tiefensondierung, Deutsche Akad. Wiss., Berlin.

Wingate, M.; Pisarevsky, S.: Evans, D., 2002. Rodinia connections between Australia and Laurentia: no SWEAT, no AUSWUS. Terra Nova, 14(2), 121-128.

Woods, D.; Lilley, F.: 1979, Geomagnetic induction in central Australia. Journal of Geomagnetic and Geoelectricity, 31, 449-458.

Woods, D.; Lilley, F.: 1980, Anomalous geomagnetic variations and the concentration of telluric currents in south-west Queensland, Auslralia. Geophysical Journal of the Royal astronomical Society, 62, 675-689.

Wyllie, M.R.J.: 1957, The Fundamentals of electric Log Interpretation. Academic Press, New York, N.Y.,176pp.

Zhang, P., Pedersen, L.B., Mareschal, M., and Chouteau, M.: 1993, Channelling contributions to tipper vectors: a magnetic equivalent to electrical distortion. Geophys. J. Int.(1993) 113,693-700.

Zhang, P., Roberts, R \& Pedersen, L.B.:1987, Magnetotelluric strike rules. Geophysics, 52 267-278. 


\section{Acknowledgements}

I owe my deepest gratitude to many people who supported me in one way or the other in the realisation of this project. Top on this list are Prof. Dr. Hans Hofsäss (Dean, faculty of physics), Prof. Dr. Cynthia Volkert (Co supervisor) \& Prof. Dr. Andreas Tilgner (Academic Dean) who all stood by me during difficult times to chart a way forward. I believe God touched and directed them to act the way they did. Closely followed on this list and equally well appreciated is Prof. Dr. Andreas Hördt (my supervisor) from TU Braunschweig. He readily accepted to be my supervisor and did a great job too. His thorough reading of the manuscript and critical suggestions helped me so much to accomplish this thesis. I wish also to appreciate and hold in high esteem Dr. Martin Leven for constant advice and moral support especially when things were not going smoothly. I must not leave out Prof. Dr. K. Bahr \& Dr. F. Simpson who measured \& provided the data analysed in this Thesis; they were equally helpful at the early stages of my work. Equally to be mentioned is the management of Alice Spring observatory who provided continuous magnetic data that were very useful in the GDS analysis.

Many people at the institute of Geophysics helped me with some aspects of geomagnetic depth soundings (GDS) and modelling of 3D data. Here I would like to thank Felix Hippmann whose ideas in GDS were very helpful. His experience in Generic Mapping Tools (GMT), a software to present data was equally very useful to me. I equally had support from Mark Sakschewski, Djamil Al-Halbouni \& Ulrich Einecke (technical support) older members that I met at the institute. My thanks too to Reiner Hannings and Manfred Herden who were always ready to give assistance where it was within their reach. My friend Daniel Mokobi is appreciated for reading some chapters of the thesis and making valuable suggestions.

Last but very importantly, I thank my wife and children who kept me in constant prayers.

This work was supported by Deutsche Forschungsgemeinschaft (German Research Foundation) (Grant nr. GZ: SI 856/2-1) and the Faculty of physics, Georg-August-Universität Göttingen. 


\title{
Curriculum Vitae
}

\author{
Personal Data
}

\author{
Name: $\quad$ Lawrence Teku Fon \\ Born: $\quad 06 / 12 / 72$ in Bessi-Batibo (Cameroon), Nationality: Cameroon \\ Marital Status: $\quad$ Married to Fon Caro. We have 3 Kids (Kelly-September 2000; \\ - $\quad$ Clovis-July 2002 \& Teku Junior-August 2007)
}

\section{Education}

\author{
1978-1985 \\ $1985-1990$ \\ FSLC, G.S Upper Bessi. \\ 1990-1992 \\ 1993-1997 \\ Ordinary level General certificate of education (GCE O/L), \\ GSS Batibo. \\ Advance Level General certificate of education (GCE A/L), \\ CCAST Bambili. \\ 2004-2007 \\ Bachelor of Science (B.Sc.) in Physics/minor in Computer \\ Science, University of Buea, Cameroon. \\ M.Sc. in Physics, University of Siegen, Germany.

\section{Professional Experience} \\ 1997-1999 Physics \& Mathematics teacher, Cameroon Protestant College \\ (CPC) Bali. \\ 1999-2001 In-service training program of the Pressbyterian Education

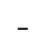 \\ $-$ \\ 2001-2004 \\ $2002-2004$ \\ $2005-2006$ \\ 2007-2008 \\ $-$ \\ $2007-2008$ \\ $-$ \\ 2008-2011 \\ Authority (PEA) and the Baptist Education Authority in \\ collaboration with the German Development Service (Deutscher \\ Entwicklungsdienst (DED)). \\ Teacher/Head of the physics department, Pressbyterian \\ Comprehensive High School (PCHS) Kumbo, Cameroon. \\ Science Coordinator, PCHS Kumbo, Cameroon. \\ Research Assistant, University of Siegen, Germany. \\ Technical services (Technical department), Christoffer Haus, \\ Siegen. \\ English Teacher, German Employee Academy (Deutsche \\ Angestellten Akademie, DAA) Siegen \\ Science member of Staff \& PhD research Student, \\ Georg-August-Univesität Göttingen
}

In addition to research at the Graduate School of Physics at the institute of Geophysics, Georg-August-Univesität Göttingen, also participated in limited teaching, through correction and discussion of Tutorials with Physics undergraduate students. Participated in the correction of end of semester examinations for the courses that I was a Tutor. Supervised and corrected protocols of undergraduate physics practicals. 